Merci d'utiliser le titre suivant lorsque vous citez ce document :

Nielson, J. et D. Taglioni (2004-03-29), « Libéralisation des échanges de services : Identification des possibilités et des avantages ", Éditions OCDE, Paris.

http://dx.doi.org/10.1787/750376550000

\title{
Libéralisation des échanges de services
}

\section{IDENTIFICATION DES POSSIBILITÉS ET DES AVANTAGES}

\author{
Julia Nielson \\ Daria Taglioni
}

La version originale de ce document a été publiée comme suit :

Nielson, J. and D. Taglioni (2004-02-06), "Services Trade Liberalisation: Identifying Opportunities and Gains", OECD Trade Policy Papers, No. 1, OECD Publishing, Paris. http://dx.doi.org/10.1787/710267064875 
DIRECTION DES ECHANGES

Français - Or. Anglais

COMITE DES ECHANGES

Groupe de travail du Comité des échanges

LIBÉRALISATION DES ÉCHANGES DE SERVICES : IDENTIFICATION DES POSSIBILITÉS ET DES AVANTAGES

Document de travail de l'OCDE sur la politique commerciale $n^{\circ} 1$

Julia Nielson et Daria Taglioni

Les documents de travail sur la politique commerciale sont maintenant disponibles sur le site Internet de l'OCDE : http://www.oecd.org/trade

JT00161064

Document complet disponible sur OLIS dans son format d'origine Complete document available on OLIS in its original format 


\section{AVANT-PROPOS \\ Libéralisation des services : identification des possibilités et des avantages}

Cette étude comporte deux volets : l'identification d'exemples concrets d'exportations de services par les pays en développement, et des études quantitatives sur les avantages de la libéralisation. Loin d'être exhaustive, elle est limitée à bien des égards, mais l'on peut néanmoins en dégager deux conclusions essentielles. Tout d'abord, comme l'illustre la première partie de l'étude, il apparaît clairement que le secteur des services présente pour les pays en développement un très grand intérêt du point de vue des exportations par-delà le mode 4 (mouvements temporaires de personnes physiques fournissant des services), dans la mesure où ces pays sont des acteurs mondiaux ou régionaux dans des secteurs comme les services aux entreprises (externalisation), les services portuaires et services de transport maritime, les services audiovisuels, les services de télécommunications, les services de construction et les services de santé. Deuxièmement, comme on le voit dans la seconde partie de l'étude, pour la plupart des pays dont beaucoup de pays en développement, les gains liés aux exportations résultant de la libéralisation du marché ne sont ni la seule ni la plus importante source de gains attendus. Les avantages de la libéralisation des services découlent, pour une bonne part, non de la recherche d'un meilleur accès aux marchés à l'étranger mais de l'accroissement de la compétitivité et de l'efficience du marché intérieur. Ensemble, les deux observations de cette étude mettent en évidence les avantages potentiels de la libéralisation du marché des services tant pour les pays développés que pour les pays en développement.

Mots clés : services, exportations, pays en développement, obstacles avantages, libéralisation, équilibre général calculable.

\section{REMERCIEMENTS}

Cette étude a été réalisée par Julia Nielson et Daria Taglioni de la Division « Liens en matière de politique commerciale » de la Direction des échanges, sous la supervision de Ken Heydon, Directeur adjoint de cette Direction. Le Secrétaire général a accepté de déclassifier le document, sous sa responsabilité, conformément à la recommandation du Groupe de travail du Comité des échanges, afin de diffuser les informations sur ce sujet auprès d'un public plus vaste.

S'agissant de la première partie, les auteurs sont reconnaissantes de l'aide apportée par les organisations et gouvernements suivants pour trouver des contacts et diffuser l'enquête auprès des entreprises et associations intéressées : Centre du commerce international (ITC), Réseau de l'entreprise en Afrique de l'Ouest (REAO), Comité consultatif économique et industriel auprès de l'OCDE (BIAC), Nippon Keidanren, Forum européen des services, US Coalition of Service Industries, Hong Kong Coalition of Service Industries, Chambre de commerce internationale, et gouvernements de l'Argentine et de la Colombie. Les auteurs expriment également leur gratitude envers Evalueserve et la Conférence des Nations unies sur le commerce et le développement (CNUCED) qui les ont aidées à trouver des exemples intéressants d'exportations de services par des pays en développement. Enfin, les auteurs souhaitent remercier toutes les entreprises et personnes ayant répondu à l'étude de l'OCDE.

Concernant la seconde partie, les auteurs tiennent à remercier John Walley et Philippa Dee pour leur contribution, ainsi que Nora Dihel, Giuseppe Nicoletti, Patrick Jomini et Wolfgang Hubner pour leurs points de vue et commentaires précieux.

\section{Copyright OCDE 2004}

Les demandes de reproduction ou de traduction totales ou partielles de cette publication doivent être adressées à : Service des publications, 2 rue André-Pascal, 75775 Paris Cedex 16, France 


\section{TABLE DES MATIÈRES}

RÉSUMÉ

PARTIE I : EXEMPLES D’EXPORTATIONS DE SERVICES DES PAYS EN DÉVELOPPEMENT.... 13

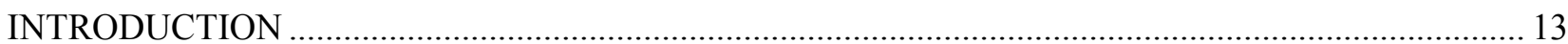

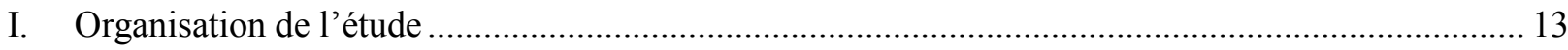

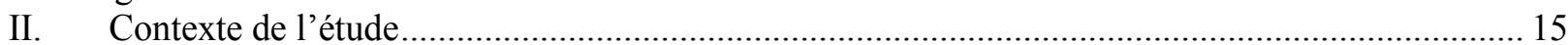

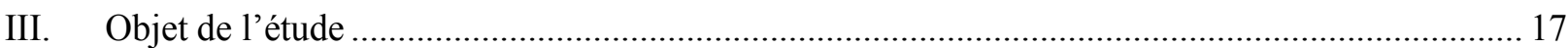

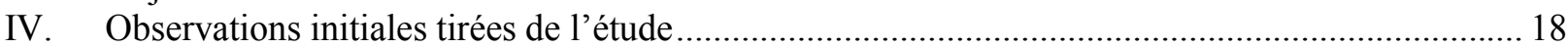

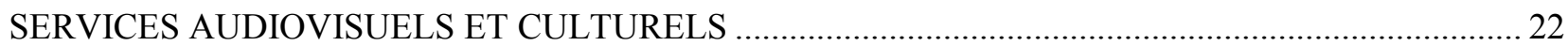

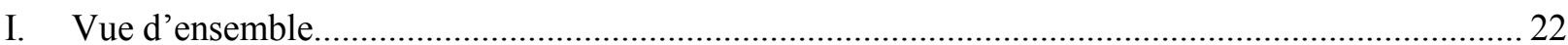

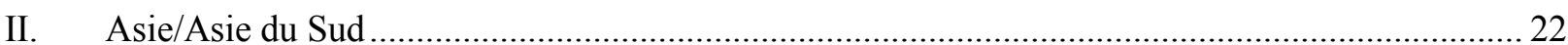

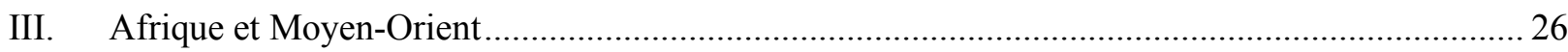

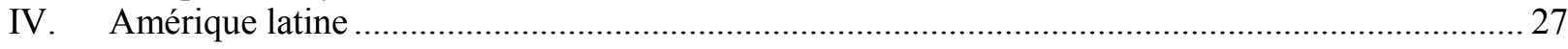

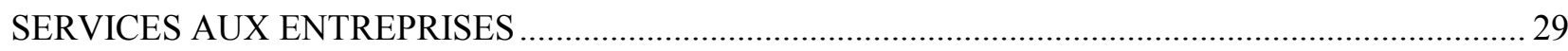

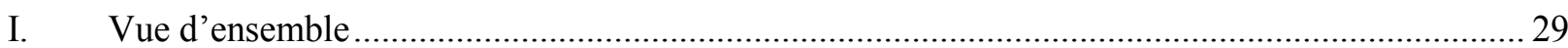

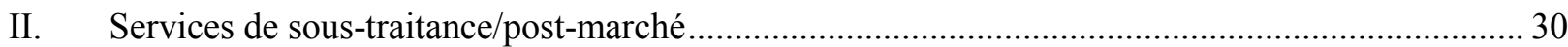

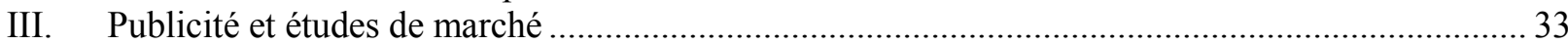

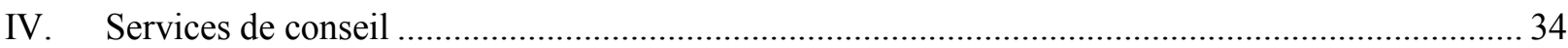

V. Autres services aux entreprises et appui technique ................................................................ 35

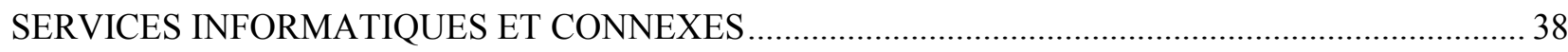

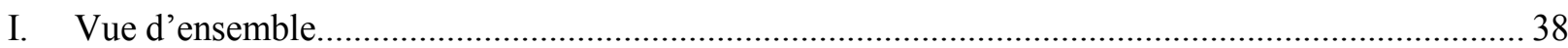

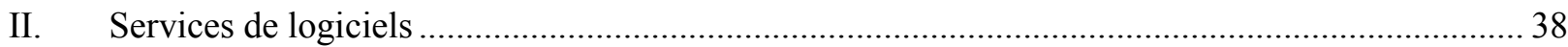

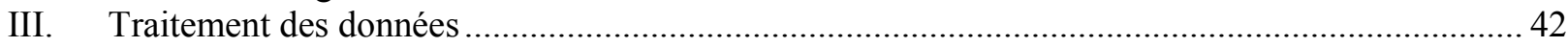

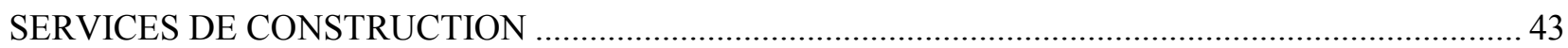

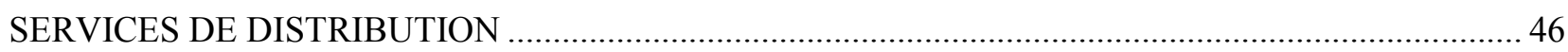

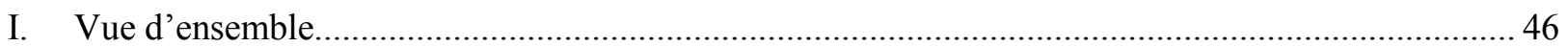

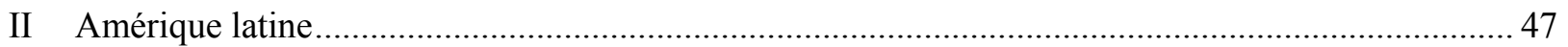

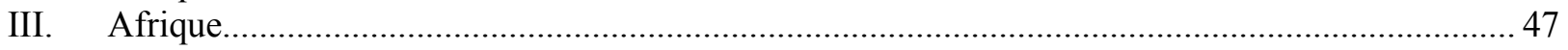

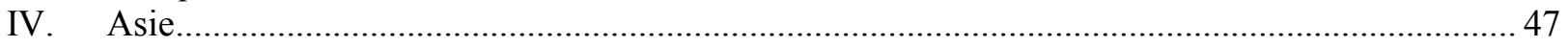

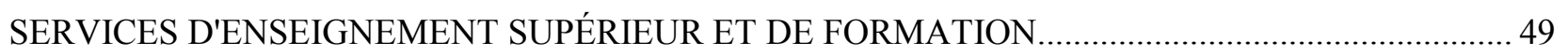

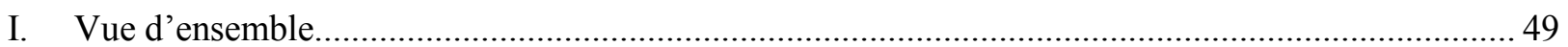

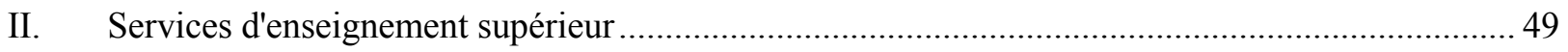

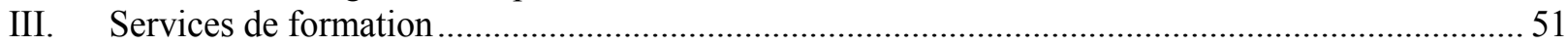

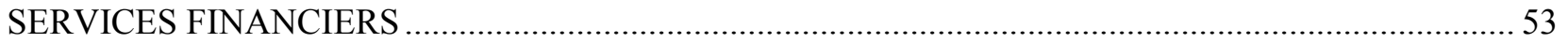




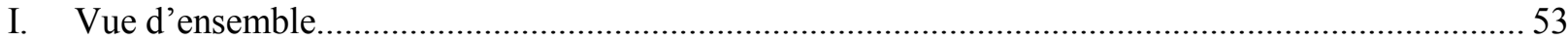

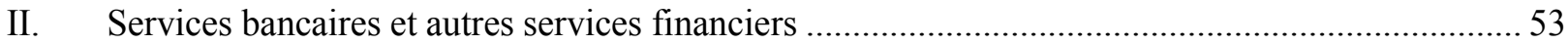

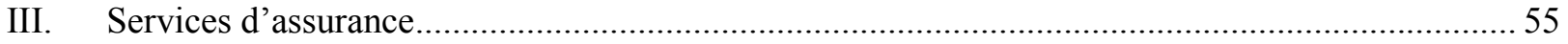

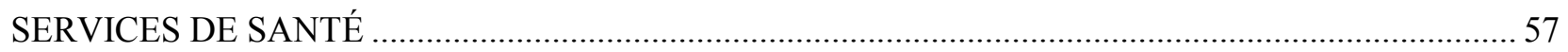

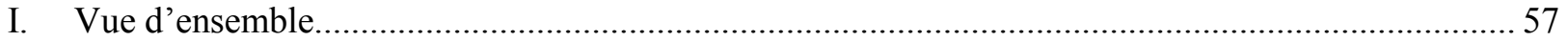

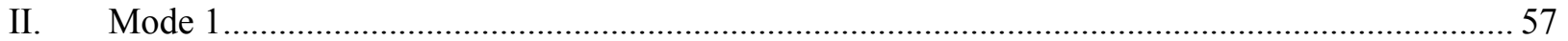

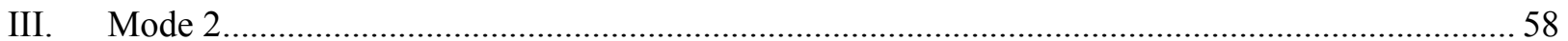

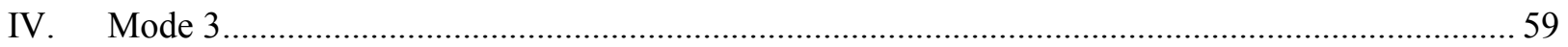

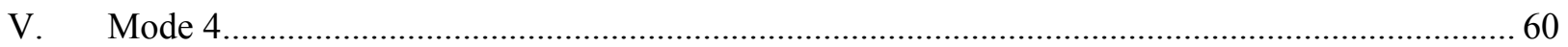

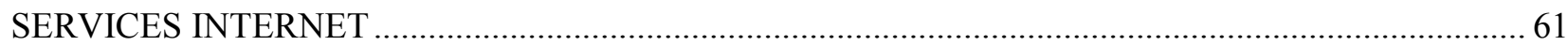

SERVICES PORTUAIRES ET CONNEXES, SERVICES DE TRANSPORT MARITIME .................... 63

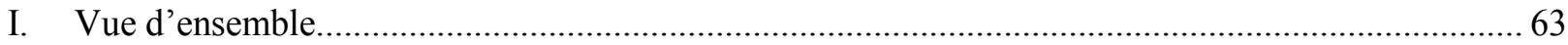

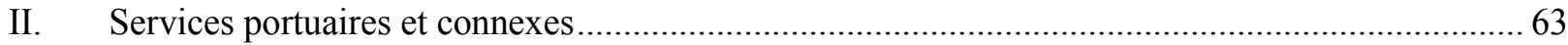

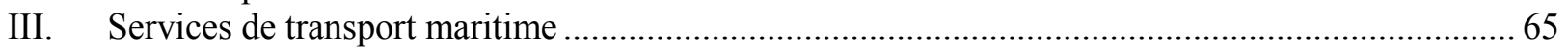

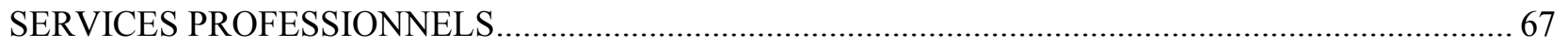

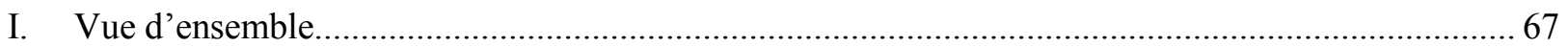

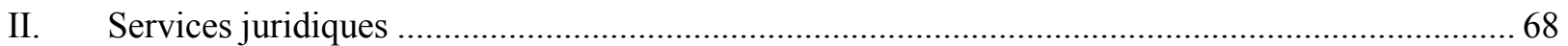

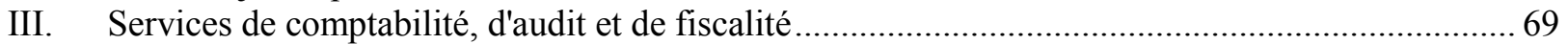

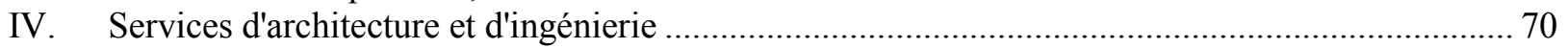

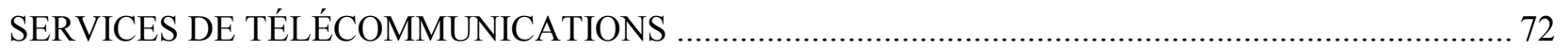

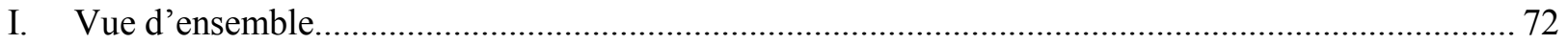

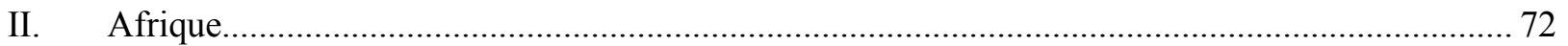

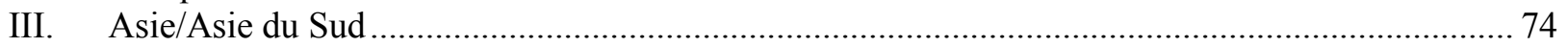

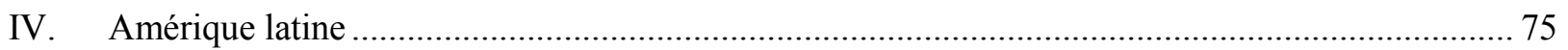

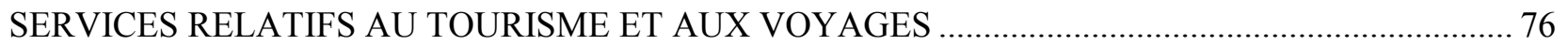

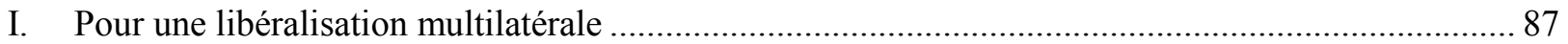

II. Sujets de préoccupation dans les pays en développement : quelques exemples .......................... 87

PRINCIPALES CONCLUSIONS DES ÉTUDES DISPONIBLES ...................................................... 90

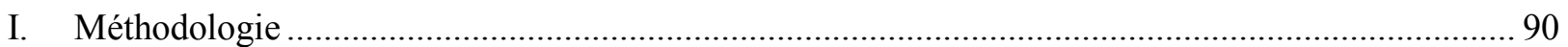

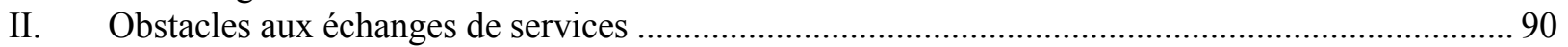

Mesures tenant compte essentiellement de la fréquence des obstacles ............................................. 91

Mesures quantitatives de la restrictivité des obstacles au commerce ……......................................... 94

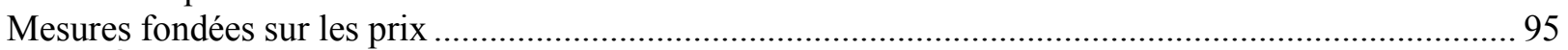

III. Éventail des résultats et méthodologie de base pour l'élaboration de modèles calibrés du

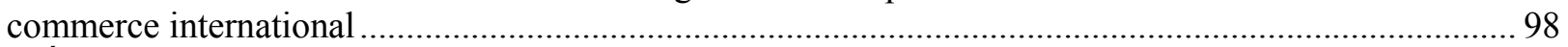

Études modélisant les services sous la forme d'échanges transfrontières (mode 1 de fourniture de

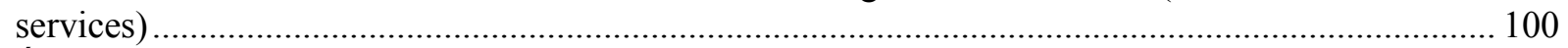
Études dont la structure de modélisation traite séparément les flux d'IDE, les activités de filiales étrangères et les mouvements temporaires de personnes (modes 3 et 4 de fourniture de services) .... 102

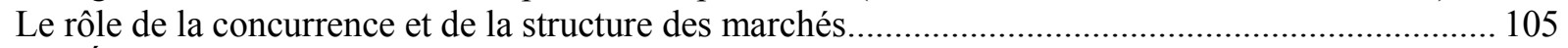

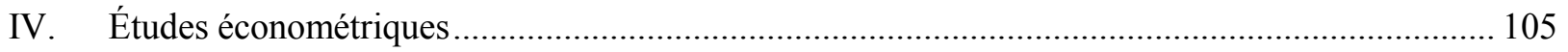

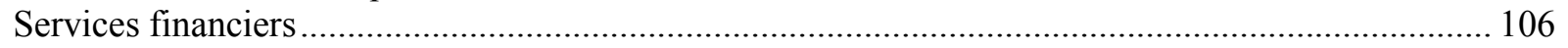

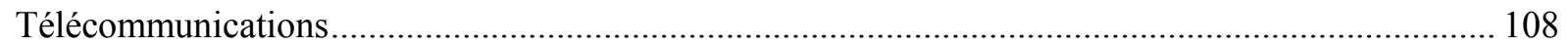

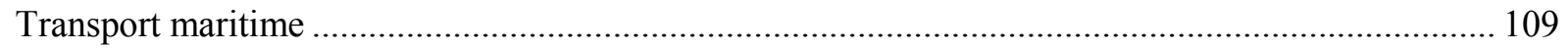

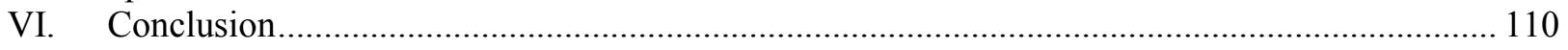


I. Caractéristiques distinctives des services : différences entre services et biens

Importance de l'accès au marché et du traitement national : renforcement de la concurrence intérieure et amélioration de l'efficacité de la production.....

Le problème des services ayant un rôle d'intermédiaires................................................................. 116

Identification et mesure des obstacles et des réglementations concernant les services ..................... 116

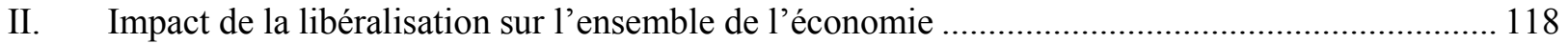

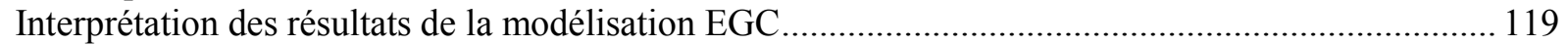

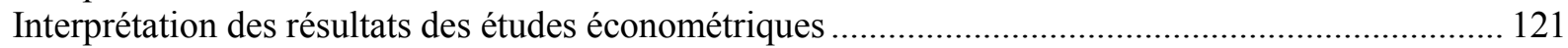

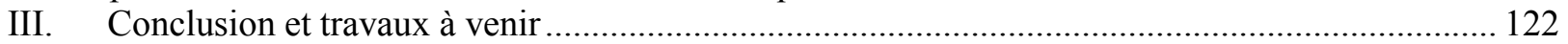

Appendice 1. Comment mesurer les obstacles aux échanges de services............................................... 125

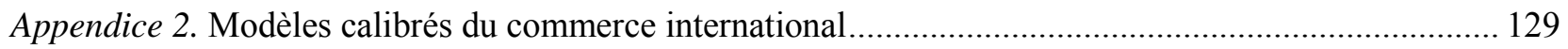

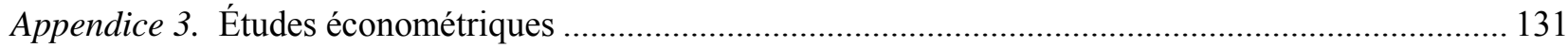

Appendice 4. Résultats de l'évaluation des restrictions touchant les échanges de services bancaires ${ }^{\mathrm{a}} \ldots . . . .132$

Appendice 5. Taux de restrictivité des échanges de services pour des secteurs particuliers, dans des économies en développement, des pays à revenu faible ou moyen (PRFM), et des pays à revenu

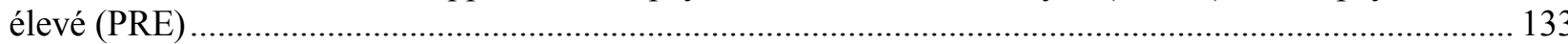

Appendice 6. Résultats d'études egc modélisant les services sous forme d'échanges transfrontières - mode 1 de fourniture de services.

Appendice 7. Résultats d'études egc modélisant séparément les obstacles à la présence commerciale mode 3 de fourniture de services - et ceux qui concernent les autres modes.

Appendice 8. Sélection d'études économétriques 138

\section{Tableaux}

Tableau 1. Exportations mondiales de services commerciaux par région, 1990-2001 ......................... 16

Tableau 2. Tarifs de manutention des conteneurs dans les terminaux argentins..................................... 64

Tableau A1. Liste de sociétés multinationales qui sous-traitent à des pays en développement................ 80

Tableau A.2. Entreprises de pays en développement figurant dans les 150 principales sociétés de construction classées en fonction du chiffre d'affaires réalisé en dehors du pays d'origine en 2001 (en

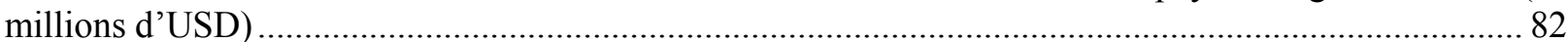

Tableau A.3. Les vingt principaux terminaux de conteneurs et leur débit en 1999 ............................... 85

Tableau A.4. Entreprises de pays en développement figurant dans les 150 premières sociétés mondiales de conception de projet (en architecture et ingénierie), classées en fonction du chiffre d'affaires réalisé

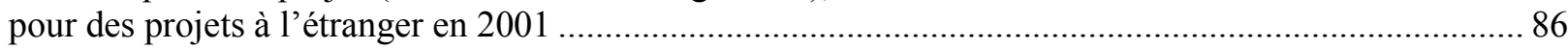




\section{RÉSUMÉ}

1. Cette étude comporte deux volets: l'identification d'exemples concrets d'exportations de services par les pays en développement, et des études quantitatives sur les avantages de la libéralisation.

2. Loin d'être exhaustive, cette étude est limitée à bien des égards, mais permet de formuler un certain nombre d'observations préliminaires. Il s'en dégage en particulier deux conclusions essentielles. Tout d'abord, comme l'illustre la partie I de l'étude, il apparaît clairement que le secteur des services présente pour les pays en développement un très grand intérêt du point de vue des exportations. Ensuite, comme le montre la partie II, les gains liés aux exportations résultant de la libéralisation du marché ne sont, pour la plupart des pays dont beaucoup de pays en développement, ni la seule ni la principale source de gains attendus. Ensemble, ces deux constatations mettent en évidence les avantages potentiels de la libéralisation du marché des services pour les pays développés comme pour les pays en développement.

3. Dans la partie I, l'étude présente un échantillon d'exportations de pays en développement dans différents secteurs : services audiovisuels et culturels ; services aux entreprises; services informatiques et services connexes ; services de construction; services de santé ; services de formation et d'enseignement supérieur; services portuaires et services de transport maritime; services professionnels; services de télécommunications et services de tourisme. Compte tenu des réalités commerciales du secteur de la fourniture de contenu et de services Internet, celui-ci est traité dans une section distincte appelée « services Internet $»^{1}$.

4. Même si plusieurs entreprises de pays en développement exportent avec succès divers services vers des pays développés et des pays en développement, il faut signaler qu'un nombre relativement limité de pays en développement semble prendre une part active aux exportations de services dans divers secteurs. Cela pourrait indiquer que les exportations de services, sur le plan international, vont de pair avec des niveaux de développement élevés et que tous les pays en développement ne sont pas encore en mesure d'être des exportateurs à grande échelle. C'est peut-être tout particulièrement le cas pour les secteurs dans lesquels les exportations semblent avoir pour base une solide demande intérieure (distribution, télécommunications, audiovisuel par exemple). Ce phénomène s'explique tout autant par la nature des échanges de services dans une économie mondialisée, caractérisée par des interrelations et des synergies puissantes entre différents secteurs de services, et par la tendance des grands acteurs mondiaux à passer des accords de fusion et d'alliance entre pays et secteurs. Le fait que les exportations de services concernent un nombre relativement limité de pays en développement est peut-être dû aussi au biais géographique inhérent à la méthode d'investigation choisie.

5. On observe aussi que, si les pays développés dominent globalement les échanges de services, les pays en développement semblent particulièrement performants dans certains secteurs tels que les services portuaires et de transport maritime, les services audiovisuels, les services de construction et les

Les Classifications de l'AGCS traitent séparément le support et le contenu, mais de nombreux fournisseurs de services Internet offrent aussi du contenu. Comme il est difficile de séparer les deux activités, elles ont été groupées dans une section spéciale sous le nom de « services Internet ». 
services de santé. Dans certains services, par exemple - activités portuaires et transport maritime - les pays en développement sont des acteurs mondiaux.

6. L'étude confirme que les pays en développement possèdent un net avantage comparatif dans les services à forte intensité de main-d'œuvre (construction, traitement des données), y compris - dans un nombre croissant de domaines - aux plus hauts niveaux de qualifications. Les avancées technologiques des secteurs des télécommunications et de l'informatique ont permis aux pays en développement dotés d'une main-d'œuvre qualifiée et compétitive sur le plan des coûts de produire et d'exporter dans le monde entier des services informatiques et des services connexes. Les résultats spectaculaires enregistrés par l'Inde ces 20 dernières années en sont l'exemple le plus remarquable et le mieux connu.

7. A cet égard, l'importance de la fourniture électronique de services pour les pays en développement apparaît clairement. La sous-traitance et les services post-marché, couvrant les services informatiques et connexes, les services aux entreprises, les services professionnels et financiers, sont les principaux domaines dont l'exportation intéresse les pays en développement. Les TIC ont créé de réelles possibilités pour de nombreux pays en développement en réduisant de façon spectaculaire le coût du transport et en renforçant ainsi leur avantage comparatif. Le coût relativement bas de la main-d'œuvre hautement qualifiée et l'amélioration des moyens de télécommunication en font de toute évidence un domaine de croissance potentielle dans l'avenir. En outre, la sous-traitance peut à la fois exploiter et contribuer à soutenir les exportations de services existantes, comme on le voit dans le secteur de l'audiovisuel, où les pays qui disposent d'une importante industrie cinématographique choisissent de plus en plus de sous-traiter la post-production de films étrangers. Pour profiter des possibilités de sous-traitance, les pays en développement devront se doter d'infrastructures modernes et efficaces, notamment pour les télécommunications et l'énergie, et avoir accès aux marchés des services du mode 1 des pays développés.

8. L'étude montre bien également l'importance des échanges régionaux, souvent entre pays en développement. Dans certains secteurs (les télécommunications, par exemple), le commerce s'effectue principalement entre pays de la région. Dans d'autres, les marchés régionaux sont importants, servant notamment de tremplin pour des activités mondiales.

9. Dans bien des secteurs, un marché intérieur dynamique joue un rôle essentiel comme plate-forme de développement de la capacité d'exportation. Cela est manifeste dans les télécommunications, l'audiovisuel, la distribution, les services portuaires et connexes et l'enseignement supérieur - et même, dans certains cas, dans les secteurs liés au commerce électronique (ainsi, un exportateur de solutions de commerce électronique a signalé qu'il était difficile de démontrer aux acheteurs étrangers l'efficacité des solutions qu'il avait mises au point dans la mesure où il ne pouvait les tester que sur un marché intérieur étroit). La démarche est généralement la suivante : les entreprises alimentent d'abord le marché intérieur et attendent d'avoir atteint la taille critique pour commencer à exporter dans les pays de la région et/ou dans ceux, comme l'ancien colonisateur, avec lesquels existent des liens historiques forts. Enfin, elles peuvent devenir des acteurs du marché mondial. Les exceptions à cette évolution générale sont les services dépendant des TIC et les services connexes, pour lesquels la situation géographique a moins d'importance, ainsi que le tourisme, secteur qui permet à une société de viser dès le départ le marché mondial.

10. Le schéma « activités intérieures-régionales-mondiales » de développement des échanges connaît une variante, dans les cas où une capacité d'exportation mondiale a été développée par des services importés. Par exemple, si, dans les services financiers, l'étude n'a guère trouvé de cas d'entreprises nationales de services financiers de pays en développement qui exportent des services, elle a trouvé des 
filiales d'entreprises de pays développés implantées dans ces pays qui exportaient, tant à destination du pays d'origine de la société mère qu'à destination d'autres pays, développés et en développement ${ }^{2}$.

11. De fait, l'étude souligne l'importance des liens entre les entreprises pour le développement d'une capacité d'exportation - en particulier des partenariats avec des entreprises de pays développés, mais aussi avec des entreprises d'autres pays en développement. Très peu d'exportateurs sont des entrepreneurs individuels. Les travaux que mène actuellement l'OCDE sur la libéralisation des échanges de services dans les pays d'Europe du Sud-Est montrent que l'un des facteurs qui influent sur les performances du secteur bancaire est le niveau de participation étrangère, qui peut apporter une amélioration des compétences et des pratiques professionnelles.

12. Les liens régionaux, linguistiques/culturels et historiques (par exemple entre les anciennes colonies et leurs colonisateurs) peuvent aussi jouer un rôle important dans les services aux entreprises. Les entreprises dont les activités débordent le marché intérieur exportent souvent d'abord vers les pays avec lesquels ce type de liens existe, les diasporas se révélant importantes dans des domaines qui vont de la médecine traditionnelle aux services audiovisuels. De fait, des créneaux réels pourraient être exploités dans les cas où les facteurs culturels et géographiques déterminent la réussite de la commercialisation d'un produit - on peut citer comme exemple le parc à thème agricole créé en Colombie, qui exporte maintenant son concept vers des pays en développement et développés, ainsi que les remèdes traditionnels d'un certain nombre de pays asiatiques et plusieurs initiatives en matière d'écotourisme. Les pays développés ayant des liens historiques et coloniaux avec certains pays en développement peuvent aussi représenter une source de capitaux et de crédibilité pour les exportateurs des pays en développement.

13. Cependant, l'étude montre également que, dans un certain nombre de secteurs, il existe peu d'exemples d'entreprises de pays en développement qui exportent (secteurs des services énergétiques ${ }^{3}$ et environnementaux par exemple). Cette situation peut être due à l'importance des coûts fixes à supporter pour pénétrer ces secteurs, et à l'existence de quelques très grandes entreprises présentes dans le monde entier. Même dans les secteurs où les pays en développement exportent, l'étude révèle aussi un certain nombre de problèmes communs auxquels leurs exportateurs doivent faire face :

- Manque d'accès au financement pour l'exportation ou le développement de l'entreprise :

- Les exportations de services des pays en développement concernent souvent des domaines où les besoins en capitaux sont relativement faibles, ou offrant la possibilité d'accéder aux capitaux par le biais de partenariats internationaux.

2 Cette remarque souligne une autre difficulté de l'étude; à l'ère des sociétés transnationales, dotées de filiales et de succursales dans de nombreux pays, tandis que les fusions et acquisitions représentent une large part des flux d'IDE, parler de la nationalité d'une société ne va pas de soi ; ainsi, le $13^{\text {ème }}$ port le plus actif du monde se trouve au Royaume-Uni (Felixstowe), mais il est possédé et géré par une entreprise de Hong Kong.

3 Il existe cependant des exceptions. Ainsi, Grupo Enersis est une compagnie d'électricité du Chili qui assure la production, le transport et la distribution d'électricité au Chili, en Argentine, au Brésil, en Colombie et au Pérou. Avec plus de 10 millions de clients, c'est la plus grande compagnie de distribution d'électricité d'Amérique latine, et c'est aussi, par l'intermédiaire d'Endesa-Chili, sa filiale consolidée la plus importante, la première entreprise privée de production d'électricité d'Amérique latine en termes de capacité installée. Pendant les six années qui se sont achevées le 31 décembre 2002, Grupo Enersis a occupé la première place parmi les entreprises privées du Chili, sur la base des actifs et du bénéfice d'exploitation consolidés, qui atteignaient respectivement 12621.2 milliards de CLP et 532.6 milliards de CLP en 2002. Endesa-Espagne, première compagnie de production et de distribution d'électricité d'Espagne, possédait $65 \%$ des actions en circulation le 31 décembre 2002. 
- Du fait du niveau peu élevé de revenu sur le marché intérieur, il est parfois difficile pour les entreprises de se constituer dans leur pays une clientèle qui joue le rôle de plate-forme d'exportation.

- Difficulté d'établir une crédibilité auprès des fournisseurs internationaux, en particulier, mais pas seulement, dans les domaines de haute technologie :

- l'idée de l'infériorité des fournisseurs de services des pays en développement reste bien ancrée dans certains secteurs, y compris sur les marchés intérieurs de ces pays. Cela peut créer un cercle vicieux - ou vertueux; le manque de crédibilité fait baisser le nombre de clients et la réduction de la clientèle réduit la possibilité d'apporter la preuve de sa fiabilité. Il peut aussi arriver, lorsqu'un secteur commence à établir sa réputation dans son pays, que la crédibilité et la reconnaissance qui en résulte se renforcent d'elles-mêmes et conduisent à la création de réseaux locaux d'exportateurs (c'est le cas pour les TIC en Inde, le traitement des données à la Barbade, les services de santé à Cuba). Les problèmes de crédibilité sont résolus, dans certains cas, par l'établissement de partenariats avec des entreprises internationales.

- Manque d'accès à une infrastructure fiable et peu onéreuse

- La médiocrité des services de télécommunication a des répercussions sur le potentiel d'exportation de services de sous-traitance ou de post-marché, tandis que l'absence d'accès fiable à l'énergie et au transport augmente les coûts pour tous les secteurs de services.

- La médiocrité de l'infrastructure peut aussi limiter la capacité d'une entreprise de fournir un service fiable et aggraver ainsi le problème de crédibilité auprès des clients (certaines entreprises déclarent ainsi avoir perdu des clients étrangers en raison de retards dans la fourniture de services dus à la lenteur ou au manque de fiabilité des liaisons Internet).

- Manque d'accès à un éventail de réseaux formels et informels et aux facilités institutionnelles nécessaires pour le commerce :

- Ces réseaux peuvent inclure tout ce qui va d'un cadre juridique sain pour les entreprises, à des liens avec d'autres exportateurs ou associations d'exportation ou à des réseaux d'entreprises plus larges.

- Pour les services en particulier, étant donné l'étroitesse des liens et les relations symbiotiques qui existent entre les secteurs de services, des problèmes pourraient se poser pour les entreprises de services du fait de la non-disponiblité de services auxiliaires ou de soutien importants sur le marché intérieur. Une société de conseil située dans un pays en développement a déclaré avoir recours à des entreprises étrangères (souvent concurrentes) pour obtenir des services de soutien importants.

14. Enfin, il convient aussi de noter que si l'étude a permis d'identifier un large éventail d'exportations de services des pays en développement, ces exportations ne représentent pour eux, comme pour certains pays développés, qu'une part relativement peu importante de leurs échanges en comparaison des exportations de produits manufacturés et agricoles. Cependant, les exemples fournis dans cette étude montrent qu'il est encore possible d'accroître les exportations de services des pays en développement.

15. La partie II de l'étude complète ces exemples concrets par une évaluation critique des avantages macroéconomiques de la libéralisation des services, avec des études quantitatives modélisant ces 
avantages. Elle fait le bilan des travaux consacrés à des estimations des obstacles aux échanges de services et aux gains de bien-être à attendre de la libéralisation des services, estimés à l'aide de calculs économétriques et de simulations calibrées, ainsi que dans le cadre d'études sectorielles. Cette partie envisage séparément les gains pour les pays développés et pour les pays en développement, et présente une analyse comparative des obstacles aux échanges de services, des gains de bien-être et des résultats sectoriels dans les pays développés et les pays en développement, qui fait aussi ressortir les variations selon les secteurs ou les modes de fourniture. L'étude explore en outre les problèmes méthodologiques généraux qui se posent, en se demandant notamment dans quelle mesure la méthode utilisée peut expliquer les différences dans les résultats obtenus (et comment elle influe sur la fiabilité de ces résultats).

16. Les restrictions aux échanges de services entraînent des coûts, qui se traduisent habituellement par des prix plus élevés pour les entreprises et les consommateurs. Elles limitent la concurrence intérieure et internationale, réduisent l'efficience et permettent aux fournisseurs en place de majorer leurs prix par rapport à ceux d'un marché concurrentiel. En estimant dans quelle mesure les restrictions augmentent les prix ou entravent la concurrence, on fait ressortir les avantages de la levée de ces restrictions pour les consommateurs, les autorités gouvernementales et les négociateurs commerciaux.

17. Toutes les études examinées arrivent à la conclusion que, quels que soient le secteur analysé et la méthodologie utilisée, il y a en moyenne davantage d'obstacles restrictifs dans les pays en développement que dans les pays développés.

- Les télécommunications et les secteurs bancaire et financier se caractérisent par des obstacles élevés dans les pays en développement et peu élevés dans les pays développés.

- Dans un certain nombre d'autres secteurs (éducation, transport maritime, services professionnels et distribution), la différence entre pays en développement et pays développés est moins nette.

- De même, les mesures des obstacles fondées sur les prix ne font pas ressortir clairement une corrélation entre le niveau des obstacles estimés et les indicateurs du bien-être et de la production.

18. A ce jour, la plupart des études indiquent que la libéralisation des services procurera probablement des avantages importants aux pays dans lesquels les obstacles au commerce sont élevés au départ. Par conséquent, les pays en développement, où les obstacles restrictifs sont, en moyenne, plus nombreux que dans les pays développés, devraient être, à long terme, ceux qui tireront le plus de profit de la libéralisation des services. Les plupart de ces avantages découlent de la libéralisation du secteur national des services et non de la recherche d'un meilleur accès aux marchés de services étrangers. A court et à moyen terme, cependant, les coûts d'ajustement de l'élimination des obstacles et du retour à la réglementation risquent d'influer négativement sur ces avantages. Ils seront probablement plus difficiles à supporter dans les pays en développement, selon le secteur libéralisé.

- Les ajustements faisant suite à la libéralisation des échanges, bien que délicats, peuvent être facilités par l'adoption de cadres stratégiques adéquats; en outre, certains éléments semblent indiquer que les coûts d'ajustement sont plus faibles pour les services que pour les secteurs de production. Dans ce contexte, il sera essentiel de bien prendre en compte la nature, le rythme et la succession des étapes de la libéralisation pour contrôler l'ajustement et s'assurer que la libéralisation s'appuie sur des cadres réglementaires solides. Cette condition est inscrite dans la structure de l'AGCS lui-même, qui prévoit des possibilités systématiques de flexibilité et met l'accent sur une libéralisation progressive. Cependant, pour les pays les moins avancés et beaucoup de pays en développement, la création de cadres réglementaires adaptés à la libéralisation - et la capacité de les faire respecter - peut nécessiter un renforcement important des capacités. 
19. On constate aussi que les avantages de la libéralisation du commerce des services sont cinq fois supérieurs à ceux de la libéralisation des échanges de marchandises. Les estimations varient cependant en fonction de l'importance des obstacles initiaux, des cadres théoriques, des techniques de modélisation et des séries de données utilisées. Il ne paraît donc pas judicieux de considérer un chiffre ou une fourchette précis comme représentatif des avantages potentiels de la libéralisation des échanges.

20. Tout l'éventail des degrés d'intervention initiale des pouvoirs publics trouve son reflet dans la diversité des effets de la libéralisation des services sur le bien-être obtenus avec des modèles d'équilibre général. Les études utilisant les estimations approximatives ("guesstimates ») de Hoekman pour les interventions initiales font apparaître généralement des gains de bien-être importants. En revanche, les études employant les estimations calculées à partir des mesures d'impact sur les prix ou les volumes donnent généralement des gains de bien-être moins importants, quoique non négligeables.

21. L'ampleur des avantages tirés de la libéralisation des échanges dépend de la nature des obstacles et des autres restrictions des échanges. Lorsque les restrictions sont créatrices de rentes, elles font monter les prix, qui dépassent les coûts, créant ainsi des rentes dont bénéficient les producteurs. La libéralisation des restrictions créatrices de rentes peut ainsi conduire à un important transfert des prestataires vers les utilisateurs de services, le gain net pour l'économie restant relativement modeste. En revanche, lorsque les restrictions dont monter les coûts, elles faussent les conditions du marché et sont responsables de modes de production inefficaces et coûteux. La libéralisation des restrictions qui provoquent une majoration des coûts peut se traduire par un gain pour les prestataires de services comme pour les utilisateurs, et globalement par un gain relativement important pour l'économie. Ainsi, l'élimination des restrictions qui entraînent une majoration des coûts peut avoir un meilleur « rendement ».

22. On obtient des gains plus élevés, mais inégalement répartis selon les pays, lorsqu'on modélise explicitement la mobilité des capitaux et l'IDE et que l'on reconnaît le caractère imparfait de la concurrence. Il importe de signaler qu'apparemment les effets importants obtenus à l'aide de modèles ne découlent pas forcément de la libéralisation des échanges de services en soi, mais de la libéralisation supposée des marchés de facteurs qui l'accompagne. Cette observation est parallèle à celle qui concerne les travaux sur les échanges de marchandises, où la libéralisation des marchés de facteurs qui accompagne la libéralisation du commerce des marchandises produit parfois, au plan international, des effets importants et inégaux selon les pays.

23. Les études qui traitent explicitement l'investissement direct étranger comme un mode de fourniture de services indiquent que la libéralisation des échanges de services augmente le revenu mondial global mais que certains pays voient leur bien-être diminuer légèrement. Les pertes de bien-être peuvent avoir trois origines. Premièrement, la levée des restrictions à l'investissement étranger peut détourner les capitaux vers des pays qui, auparavant, avaient des obstacles à l'investissement relativement élevés. Deuxièmement, les obstacles à l'entrée génèrent des rentes, dont certaines vont aux détenteurs de capitaux étrangers. Avec la libéralisation, ces rentes sont érodées par la concurrence et les pays qui sont d'importantes sources d'investissement direct étranger peuvent donc être perdants. Enfin, il y a des effets sur les termes de l'échange, qui seront examinés plus loin.

24. Les études centrées sur les mouvements des personnes qui fournissent des services montrent qu'il s'agit d'un moyen de livraison essentiel pour les pays exportateurs. Comme c'est le cas avec les études analysant la mobilité des facteurs et l'IDE, il semble que la libéralisation concomitante supposée des marchés de facteurs soit la principale source de gains importants. Cependant, les travaux sur le mode 4 ne vont pas sans difficultés, en particulier parce que la plupart des pays exigent que les travailleurs étrangers soient payés au même tarif que les ressortissants nationaux - ce qui atténue l'avantage des fournisseurs des pays en développement sur le plan des coûts. Ne pas reconnaître ce fait peut limiter la pertinence des études quantitatives faisant état de gains attribuables à la mobilité accrue de la main- 
d'oeuvre. De même, les études ne tiennent pas toujours suffisamment compte des cadres réglementaires existants qui régissent les marchés du travail, notamment en ce qui concerne la rémunération, les conditions de travail et la reconnaissance des qualifications.

25. Les études économétriques analysant les effets dynamiques de la libéralisation et son incidence sur des secteurs particuliers aboutissent à des gains plus importants que les simulations des modèles d'équilibre général calculable (EGC). Ce résultat est sans doute imputable à l'élément dynamique des études économétriques qui tiennent compte des ajustements à long terme s'effectuant à travers l'accumulation de capital, la croissance démographique et le progrès technologique.

26. Du point de vue méthodologique, l'étude de l'OCDE montre qu'il est important d'envisager la libéralisation des échanges de services dans un contexte général, plus large en tout état de cause que celui de la libéralisation des échanges des marchandises.

- Premièrement, la libéralisation des services ouvrira des marchés à de nouveaux fournisseurs locaux, aussi bien qu'étrangers, et aura comme conséquence notable d'entraîner des gains qui découleront non pas de la recherche d'un meilleur accès aux marchés des services étrangers, mais du renforcement de la concurrence et de l'efficience sur le plan local.

- Deuxièmement, il est essentiel, pour connaître les effets de la libéralisation des services, de déterminer si un marché véritablement concurrentiel a été créé. La transformation de monopoles publics en monopoles privés engendre des avantages négligeables et, de même, la structure de la concurrence dans l'industrie influe considérablement sur la répartition des avantages de la libéralisation. Les travaux que mène actuellement l'OCDE sur la libéralisation des échanges de services dans les pays d'Europe du Sud-Est montrent que ces pays ne retireraient vraisemblablement pas d'avantages à ouvrir leurs services de télécommunications sur réseau fixe à des capitaux étrangers sans faire disparaître dans le même temps les restrictions qui s'appliquent actuellement aux nouvelles entrées.

- Enfin, certaines études sectorielles montrent que l'ordre de succession des réformes (par exemple, la privatisation, puis l'introduction de la concurrence) peut aussi être une considération importante.

27. Une réserve s'impose. L'évaluation des obstacles aux échanges de services et la quantification des avantages de la libéralisation dans ce secteur constituent un champ d'étude assez nouveau, caractérisé par des cadres théoriques, des techniques de modélisation et des séries de données qui ont toujours besoin d'être complétées. Les résultats présentés dans la partie II de cette étude sont par conséquent soumis aux limites méthodologiques et statistiques inhérentes à l'état actuel des connaissances.

28. Malgré tout, on note un progrès continu dans l'affinage de la théorie et des techniques empiriques, tandis que des efforts sont en cours pour améliorer la base statistique des données. Du côté de la modélisation, les nouvelles études visent à représenter de façon plus exacte l'économie des secteurs de services et des échanges, notamment les divers modes de fourniture, leurs fonctions et caractéristiques distinctives et le traitement plus détaillé des secteurs de services. Du côté empirique, on introduit progressivement des estimations cohérentes de l'investissement direct étranger (IDE) et des données sur le commerce des services des filiales étrangères (FATS) aux niveaux bilatéral et sectoriel pour représenter la présence commerciale, qui constitue une part importante des échanges globaux de services. Par ailleurs, ces quelques dernières années, les milieux universitaires et des institutions internationales comme le FMI, Eurostat, l'OCDE et l'OMC se sont efforcés d'améliorer la fiabilité globale des données relatives aux échanges de services et de résoudre les problèmes bien connus de pénurie de données, de manque de concordance et de comparabilité entre les séries existantes, de désagrégation sectorielle limitée et d'absence de classification par mode de fourniture. 


\section{PARTIE I : EXEMPLES D'EXPORTATIONS DE SERVICES DES PAYS EN DÉVELOPPEMENT}

\section{INTRODUCTION}

1. Cette étude répond à une demande du Groupe de travail du Comité des échanges, formulée lors de sa réunion du 22 janvier 2003 ; le Groupe a demandé des travaux donnant des exemples concrets d'exportations de services en provenance de pays en développement. ${ }^{4}$ Les économies en transition entrent également dans le champ de l'étude.

\section{Organisation de l'étude}

2. L'étude présente des exemples pris dans diverses régions géographiques et un large éventail de secteurs des services. Elle couvre les exportations de services effectuées selon les quatre modes de fourniture décrits dans l'Accord général sur le commerce des Services (AGCS) :

- Les échanges transfrontières, dans le cadre desquels le service lui-même franchit la frontière (par exemple lorsqu'un architecte malaisien faxe un plan à un client se trouvant en Inde).

- La consommation à l'étranger, dans le cadre de laquelle le client se rend dans le pays où le service est fourni (par exemple lorsqu'un Malaisien passe ses vacances en Inde).

- La présence commerciale, caractérisée par le fait que le fournisseur établit une présence commerciale à l'étranger (par exemple, lorsqu'un cabinet d'architectes malaisien ouvre un bureau en Inde).

- Le déplacement de personnes physiques, dans le cadre duquel le fournisseur se rend temporairement dans un autre pays pour offrir ses services (par exemple, lorsqu'un architecte malaisien passe trois mois en Inde pour superviser la construction d'un bâtiment).

3. Le mode 4 est généralement considéré comme le mode de fourniture le plus important pour les exportations des pays en développement, et il reste effectivement dans certains secteurs une composante essentielle des échanges des pays en développement. Néanmoins, les exportations selon le mode 4 peuvent se traduire et être complétées par des exportations suivant d'autres modes (ainsi, les exportations de personnel indien spécialisé dans les TIC ont conduit à une prise de conscience de l'existence en Inde d'une main-d'œuvre qualifiée et au développement de la sous-traitance à des firmes indiennes - modes 1 et 2). L'histoire des exportations de services des pays en développement déborde assurément du mode 4 et l'objet de cette étude est de mettre en évidence des exemples d'exportations qui correspondent aux autres modes de fourniture. La fourniture électronique de services (modes 1 et 2) s'affirme notamment comme un domaine clé de l'activité et du potentiel d'exportation des entreprises de services des pays en développement; elle est un révélateur du rôle important des nouvelles technologies au service de la croissance des échanges de services et tout particulièrement pour faire davantage participer les PME des pays en développement.

4. Il convient néanmoins de rappeler que les modes de fourniture ont été définis dans l'optique des engagements à prendre au titre de l'AGCS, et ne sont pas des concepts d'usage courant dans le monde des

4. Dans le cadre de l'étude, on considère les «pays en développement » comme ceux qui se désignent comme tels dans le cadre des accords de l'OMC. 
affaires. Les sociétés ne distinguent pas leurs activités selon le mode de fourniture et nombre d'entre elles exportent des services en recourant à plusieurs de ces modes. A l'exception de certains secteurs, où la différenciation est relativement claire (comme les services de santé), l'étude ne cherche pas à déterminer à quels modes de fourniture correspondent les exemples d'exportations de services utilisés.

5. De même, bien que les informations relatives aux exportations soient présentées sur une base sectorielle, en se référant à la classification sectorielle des services de l'OMC (MTN.GNS.W/120, ou «W/120»), les activités de sociétés données peuvent s'exercer dans plusieurs secteurs. Le problème du classement s'est révélé particulièrement épineux pour les entreprises se livrant à la sous-traitance ou à des opérations de back-office, qui offraient souvent un ensemble de prestations relevant à la fois de la catégorie des services informatiques et connexes, de celle des services aux entreprises et même des services financiers. On a classé individuellement les sociétés dans le secteur qui correspond le mieux à leur activité première, même dans l'éventualité où elles exportent divers autres services connexes; certains chevauchements n'en sont pas moins inévitables. Un problème particulier s'est posé pour les entreprises qui ont la double vocation d'offrir des services Internet et du contenu; afin d'éviter des difficultés de classement, on les traite dans une section distincte.

6. En outre, l'étude retient une conception large des exportations de pays en développement : une exportation de services est considérée comme un service rendu par un "résident local» à un client « résident étranger », quel que soit le lieu de fourniture. De fait, le mode 3 comprend l'établissement à l'étranger de sociétés de services et, indirectement, les ventes qui résultent de cet investissement, qui, bien que ne faisant pas partie des exportations sur le plan de la balance des paiements, sont souvent une caractéristique essentielle de la présence commerciale internationale du pays d'origine de l'entreprise qui investit. De même, la filiale locale d'une société étrangère (par exemple PricewaterhouseCoopers Malaisie) peut ensuite exporter sur d'autres marchés (exportation de services par le pays hôte). Les entreprises locales sont également susceptibles de fournir des services à la filiale étrangère établie sur leur marché national, bien qu'il ne s'agisse pas d'une exportation ${ }^{5}$.

7. Pour trouver des exemples d'exportations de services de pays en développement, l'étude a puisé à plusieurs sources différentes. Aux recherches extensives sur Internet se sont ajoutés des éléments d'information apportés par la CNUCED (tirés des réunions d'experts consacrées aux échanges dans des secteurs spécifiques de services) et une brève enquête en ligne sur différentes sociétés. L'enquête a été adressée à des entreprises et associations professionnelles de pays développés et en développement. Dans ces derniers, on a demandé aux entreprises si elles exportaient et, dans cette hypothèse, vers quelles destinations. Dans les pays développés, on leur a demandé si elles recouraient à des services de prestataires situés dans des pays en développement; si elles possédaient des succursales et des filiales dans ces pays, et, dans ce cas, si leurs succursales et filiales exportaient sur d'autres marchés. On a reçu environ 90 réponses, provenant pour la plupart d'entreprises établies dans des économies en développement ou en transition.

8. L'étude présente un échantillon des exportations de pays en développement dans des secteurs sélectionnés: services audiovisuels et culturels; services aux entreprises; services informatiques et services connexes; services de construction; services financiers; services de santé; services d'enseignement supérieur et de formation; services portuaires et connexes ainsi que services de transport maritime; services professionnels; services de distribution; services de télécommunications; enfin

5. Ce n'est pas une exportation de services, car le prestataire et le consommateur du service sont considérés comme des entreprises locales. Toutefois, dans une perspective plus large, les occasions créées au bénéfice d'entreprises locales, qui peuvent aussi donner lieu à des transferts de technologie et à un renforcement des capacités, font partie des avantages des importations de services (c'est-à-dire importation selon le mode 3) ; elles sont traitées dans la deuxième partie de l'étude. 
services touristiques et en rapport avec le tourisme. Compte tenu des réalités commerciales du secteur de la fourniture de contenu et de services Internet, celui-ci est traité dans une section distincte appelée « services Internet $»^{6}$. Ce sont les principaux secteurs dans lesquels on a trouvé des exportations de pays en développement dans le délai prévu pour la réalisation de l'étude; des recherches supplémentaires pourraient permettre de faire ressortir des exemples dans d'autres secteurs.

9. L'étude n'est donc nullement exhaustive, mais a pour but de présenter des exemples d'exportations de pays en développement dans les secteurs choisis pour donner quelques indications ou une idée de base à propos des types d'activité qui se développent. On espère qu'ils pourront avoir un effet démonstratif, c'est-à-dire qu'ils ne montreront pas seulement l'existence d'échanges, mais pourront aussi servir à inspirer leur essor futur.

\section{Contexte de l'étude}

10. Les services comptent pour beaucoup dans l'économie mondiale. En 2000, la valeur totale de la production de services a été estimée à quelque 1400 milliards d'USD. Dans les pays en développement, les services ont contribué largement au produit intérieur; leur part dans le PIB va de $39 \%$ au Nigeria à 89 \% à Hong Kong Chine. Ils jouent également un rôle important dans les «pays moins avancés » (PMA). Dans les 49 pays ainsi qualifiés, la contribution moyenne au PIB des exportations de services était de $10 \%$ en 2000, certains dépassant les $50 \%$ (Vanuatu et les Maldives). Selon les études effectuées par le Centre des échanges internationaux (CNUCED/OMC) sur 20 PMA, la part des activités agricoles dans le produit intérieur brut ne dépassait celle des services que dans trois d'entre eux. De plus, les services constituaient la principale exportation de sept de ces 20 pays [Source: CNUCED et Centre des échanges internationaux].

11. Au demeurant, si la plus grande part du commerce mondial des services s'effectue entre pays développés, ils n'en ont nullement le monopole (voir Tableau 1). De 1991 à 2000, le groupe des pays en développement a plus que doublé ses exportations de services commerciaux, qui sont passées de 189 milliards à 399 milliards d'USD, leur part dans les exportations mondiales de services passant ainsi de $23 \%$ à $28 \%$ pendant ces dix années. Plus de 50 pays en transition et en développement exportent maintenant plus de 1 milliard d'USD de services par an; ils sont répartis entre l'Asie (10), l'Afrique et le Moyen-Orient (10), l'Europe (18) et l'hémisphère ouest (13). [Source: Cleveland, Douglas (2003) "Services and the Doha Development Agenda", Département du Commerce des États-Unis, Washington, D.C.]

6 Les classifications de l'AGCS traitent le support et le contenu séparément, mais de nombreuses entreprises qui offrent des services Internet fournissent aussi du contenu. Ces activités étant difficiles à séparer, on les a groupées dans une rubrique séparée consacrée aux services Internet. 
Tableau 1. Exportations mondiales de services commerciaux par région, 1990-2001

\begin{tabular}{|l|c|c|}
\hline Région & $\begin{array}{c}\text { \% des } \\
\text { exportations } \\
\text { mondiales } \\
\text { en 1990 }\end{array}$ & $\begin{array}{c}\text { \% des } \\
\text { exportations } \\
\text { mondiales } \\
\text { en 2001 }\end{array}$ \\
\hline Amérique du Nord & $\mathbf{1 9 . 3 1}$ & $\mathbf{2 0 . 5 0}$ \\
Canada & 2.34 & 2.44 \\
États-Unis & 16.97 & 18.06 \\
\hline Amérique latine & $\mathbf{3 . 7 9}$ & $\mathbf{3 . 9 9}$ \\
\hline Europe occidentale & $\mathbf{5 3 . 0 6}$ & $\mathbf{4 6 . 5 4}$ \\
Union européenne (15) & 47.18 & 41.94 \\
\hline Europe centrale et orientale & $\mathbf{\ldots . .}$ & $\mathbf{3 . 8 5}$ \\
\hline Afrique & $\mathbf{2 . 3 9}$ & $\mathbf{2 . 1 2}$ \\
\hline Moyen-Orient & $\mathbf{. .}$ & $\mathbf{2 . 2 4}$ \\
\hline Asie-Océanie & $\mathbf{1 6 . 7 9}$ & $\mathbf{2 0 . 7 5}$ \\
Australie & 1.26 & 1.08 \\
Japon & 5.28 & 4.37 \\
Nouvelle-Zélande & 0.31 & 0.29 \\
\hline Monde & $\mathbf{1 0 0}$ & $\mathbf{1 0 0}$ \\
\hline Source : adaptation des statistiques de l'OMC pour la période 1980-2001, \\
disponibles sur www.wto.org.
\end{tabular}

12. En outre, certaines estimations récentes de l'OCDE sur le commerce de services par pays partenaire donnent des indications initiales, certes imparfaites, sur les importations de services provenant de pays en développement au Japon, dans l'Union européenne et aux États-Unis ${ }^{7}$. En 2000, un peu moins de $14 \%$ des importations totales de services du Japon émanaient de trois pays asiatiques : la Corée (5.4\%), Singapour (4.2\%) et Hong Kong (4.2\%). 20.1\% avaient pour origine le «reste de l'Asie et de l'Océanie », zone qui comprend plusieurs autres pays en développement; néanmoins, ce chiffre doit être considéré comme une estimation très approximative, car il incorpore aussi les achats en Australie et en Nouvelle-Zélande. Les importations en provenance du «reste de l'Amérique » (ce qui exclut les États-Unis, mais englobe le Canada ainsi que l'Amérique centrale et l'Amérique du Sud) représentaient $9 \%$ du total ; $3 \%$ des importations venaient d'Afrique. S'agissant de l'UE, les importations de services du reste de l'Asie et de l'Océanie (excluant le Japon, mais incluant l'Australie et la Nouvelle-Zélande) représentaient $15.3 \%$ du total, alors que $11.9 \%$ avaient pour origine le « reste de l'Europe » (excluant le commerce intra-communautaire ainsi qu'avec la Norvège et la Suisse). L'Afrique représentait $6.4 \%$ des importations de services de l'UE, tandis que le «reste de l'Amérique » (Canada et États-Unis exclus) s'élevait à $5.8 \%$. Enfin, aux États-Unis, le « reste de l'Asie et l'Océanie » était à l'origine de $18.6 \%$ des importations de services; $13.2 \%$ provenaient du «reste de l'Amérique » (Canada exclu). L'Afrique se limitait à $1.4 \%$ et le reste de l'Europe (UE et Suisse exclues) contribuait à $3.3 \%$.

13. Il est plus délicat d'estimer les échanges de services entre pays en développement. Les données bilatérales dont on dispose actuellement sont rares et ne permettent pas une prise en compte satisfaisante de ces flux. Mais les enquêtes conjoncturelles, les études par pays et les simulations à partir de statistiques en rapport laissent penser que les ordres de grandeur sont sans doute significatifs dans certains secteurs et entre certains pays. De nombreuses sources montrent effectivement de façon tangible que les entreprises de pays en développement travaillent surtout pour les marchés de pays voisins, alors que peu de stratégies d'exportation de services visant le marché mondial sont couronnées de succès.

$7 \quad$ Voir Statistiques de l'OCDE sur les échanges internationaux de services : données par pays partenaire et analyse résumée 1999-2000. Il convient de noter que les statistiques sur les échanges de services par pays partenaire sont une innovation récente et que les données ne sont pas encore totalement satisfaisantes. 
14. D'une manière plus générale, il convient de garder à l'esprit que les statistiques de services sousestiment probablement les échanges en ce domaine. La place des services dans les exportations mondiales est certes passée de $15 \%$ à près de $20 \%$ au cours des vingt dernières années. Cependant, les exportations de services ne se sont accrues que de $7 \%$, alors que la proportion des services dans la production mondiale a progressé de près de $25 \%$ par an. La divergence des données incite à penser que non seulement les échanges de services sont malaisés, mais, plus encore, que ces flux ne sont sans doute pas intégralement appréhendés en l'état actuel des concepts et des méthodologies statistiques. En effet, nombre de statistiques relatives au commerce des services sont tirées des données de balance des paiements, ce qui impose des limites au calcul des échanges de services dans la perspective de l'AGCS. Un certain nombre de secteurs de services sont laissés de côté et la plupart des statistiques ne rendent compte que des échanges effectués selon les modes 1 et 2 ; les chiffres de la balance des paiements ne rendent pas compte des échanges selon le mode 3 et ne donnent qu'une estimation approximative des transactions selon le mode $4^{8}$. La mesure statistique du commerce des services sera sensiblement améliorée par l'utilisation du récent Manuel de statistique des échanges internationaux de services, mais celui-ci ne sera sans doute pleinement appliqué qu'à longue échéance?

15. Ces problèmes de calcul sont, dans une certaine mesure, inhérents à la nature même des services. Du fait de leur caractère intangible, il n'est pas facile de les définir; quand une définition a été trouvée, l'obtention des informations nécessaires sur les flux commerciaux dépend du degré de reconnaissance internationale des concepts et de la disponibilité des données, qui la limitent aussi. Comme, par ailleurs, les échanges de services n'impliquent pas nécessairement le franchissement d'une frontière, des modes importants de fourniture internationale sont difficiles à appréhender. En définitive, les entreprises de services n'ont pas forcément conscience d'être exportatrices, surtout si elles commercialisent leurs services localement à des étrangers. Les sociétés étrangères qui opèrent dans des zones de libre-échange, les représentations locales des intitutions financières internationales et (s'agissant des pays en développement) les organisations donatrices sont souvent de gros clients des entreprises et des prestataires de services locaux; néanmoins, ces derniers ne se considèrent pas toujours comme des exportateurs quand ils travaillent avec des acheteurs de ce type.

\section{Objet de l'étude}

16. Ces problèmes techniques laissent penser que les concepts statistiques et les méthodologies en vigueur aboutissent à sous-estimer le montant des services échangés sur le plan international. En recueillant des exemples de succès des pays en développement dans les exportations de services, nous espérons éclairer la nature et la dimension d'aspects du commerce des services qui ne sont peut-être pas toujours pris en compte par les statistiques actuelles.

17. Bien qu'il existe dans la quasi totalité des pays en développement des exportateurs de services qui réussissent, on ne trouve pas toujours trace de leur succès dans l'analyse traditionnelle de l'économie et des marchés. Certains gouvernements n'ont sans doute pas pleinement conscience des possibilités existantes, tandis que les organismes chargés de l'aide internationale et les sociétés potentiellement partenaires ou clientes du secteur peuvent ignorer l'expertise qu'offrent les entreprises de pays en

8 Les transactions effectuées selon le mode 3 sont mieux appréhendées par les statistiques d'échanges de services des filiales étrangères, mais seule une minorité de pays en établit (environ 20 jusqu'à présent). Les substituts utilisés dans les chiffres de balance de paiements pour comptabiliser le mode 4 - rémunération des salariés et transferts de fonds des travailleurs immigrés - sont très approximatifs et peuvent aussi bien sous-estimer que surestimer les transactions selon le mode 4 (ils incluent par exemple des personnes qui travaillent dans d'autres secteurs que les services).

9 Voir ONU, Commission européenne, FMI, OCDE, CNUCED, OMC, Manuel de statistiques des échanges internationaux de services, disponible sur le site www.wto.org. 
développement. L'ignorance de l'activité exportatrice existante est susceptible d'en brider les possibilités de croissance. De fait, certains entrepreneurs ont signalé que le défaut de crédibilité et de reconnaissance internationales constituait le principal obstacle à l'essor de leurs exportations. Il est souhaitable que les exemples présentés dans cette étude puissent contribuer à mieux faire connaître non seulement le degré de participation actuelle des pays en développement aux échanges de services, mais aussi son énorme potentiel d'accroissement.

\section{Observations initiales tirées de l'étude}

18. La part des services augmente dans l'économie mondiale, la libéralisation du secteur progresse et se heurte à des résistances, tandis que de nombreux pays en développement pensent que le commerce des services présente peu d'intérêt pour eux; tous ces facteurs expliquent l'attention accrue portée à la structure des échanges de services. La présente étude a essayé de comprendre la nature et l'importance exactes des exportations de services des pays en développement en trouvant des exemples concrets. Bien qu'elle ne prétende nullement à l'exhaustivité et soit sujette à de nombreuses limites, on peut en inférer plusieurs observations préliminaires.

19. Bien que plusieurs entreprises de pays en développement exportent avec succès divers services vers des pays développés et en développement, l'enquête montre notamment qu'il semble exister un nombre relativement limité de pays en développement fortement impliqués dans les exportations de services concernant toute une gamme de secteurs. On pourrait en conclure que, sur le plan international, les exportations de services vont de pair avec un haut niveau de développement et que tous les pays en développement n'ont pas encore la capacité d'exporter sur une grande échelle. C'est peut-être surtout vrai dans les domaines où les performances à l'exportation paraissent s'appuyer sur une demande intérieure solide (cas de la distribution, des télécommunications et de l'audiovisuel). Cet état de fait découle aussi de la nature du commerce des services dans une économie mondialisée, caractérisée par l'importance croissante des liens et des synergies entre les différentes activités, ainsi que par la propension des grands acteurs mondiaux à fusionner et à nouer des alliances dans de nombreux pays et secteurs.

20. La concentration des exportations de services dans un groupe assez réduit de pays en développement pourrait aussi s'expliquer par le biais géographique inhérent à la méthode de recherche retenue. La richesse de la documentation et des sources secondaires disponibles est très variable en fonction des secteurs et des pays ; alors que l'on dispose de sources d'informations substantielles en ce qui concerne les technologies de l'information (TI) en Inde ou le secteur de la santé à Cuba, les renseignements relatifs aux échanges de services dans la plus grande partie de l'Afrique sont souvent succincts. Le mode d'investigation, qui repose très largement sur Internet, notamment au moyen d'une enquête en ligne, privilégie également les pays et les entreprises ayant une bonne maîtrise des TI ou dont les exportations de services leur sont liées d'une manière ou d'une autre. En définitive, le nombre de réponses à l'enquête influence l'analyse ; les plus nombreuses ont émané de sociétés situées au Japon, en Colombie, en Argentine, au Kenya et à Hong Kong.

21. On peut également faire observer qu'en dépit de la domination globale des pays développés sur les échanges de services, les pays en développement semblent particulièrement performants dans certains secteurs, tels que les services portuaires et de transport maritime, l'audiovisuel, la construction et la santé. C'est ainsi qu'ils jouent un rôle mondial dans la fourniture de certains services - services portuaires et de transport maritime. Les Philippines et Hong Kong, par exemple, ont utilisé leurs puissantes infrastructures portuaires comme plaque tournante du commerce international et, sous l'impulsion du boum des exportations de l'Asie de l'est, se sont lancés dans la gestion portuaire à l'échelle internationale.

22. L'étude confirme que les pays en développement jouissent d'un net avantage comparatif pour les services à forte intensité de main-d'œuvre (construction, traitement de données), y compris - dans un 
nombre croissant de domaines - aux plus hauts niveaux de qualification. Les avancées technologiques des secteurs des télécommunications et de l'informatique ont permis aux pays en développement dotés d'une main-d'œuvre qualifiée et compétitive sur le plan des coûts de produire et d'exporter dans le monde entier des services informatiques et des services connexes. Les résultats spectaculaires enregistrés par l'Inde ces 20 dernières années en sont l'exemple le plus remarquable et le mieux connu.

23. A cet égard, l'importance de la fourniture électronique de services pour les pays en développement apparaît clairement. La sous-traitance et les services post-marché, couvrant les services informatiques et connexes, les services aux entreprises, les services professionnels et financiers, sont les principaux domaines dont l'exportation intéresse les pays en développement. Les TIC ont créé de réelles possibilités pour de nombreux pays en développement en réduisant de façon spectaculaire le coût du transport et en renforçant ainsi leur avantage comparatif. Le coût relativement bas de la main-d'œuvre hautement qualifiée et l'amélioration des moyens de télécommunication en font de toute évidence un domaine de croissance potentielle dans l'avenir. En outre, la sous-traitance peut à la fois exploiter et contribuer à soutenir les exportations de services existantes - comme dans le secteur de l'audiovisuel, où les pays qui disposent d'une importante industrie cinématographique choisissent de plus en plus de sous-traiter la post-production de films étrangers. Pour profiter des possibilités de sous-traitance, les pays en développement devront se doter d'infrastructures modernes et efficaces, notamment pour les télécommunications et l'énergie, et avoir accès aux marchés des services du mode 1 des pays développés.

24. L'étude montre bien également l'importance des échanges régionaux, souvent entre pays en développement. Dans certains secteurs (par exemple les télécommunications), le commerce est surtout de nature régionale. Dans d'autres, les marchés régionaux ont un grand rôle, notamment comme première étape vers une plus grande internationalisation des activités.

25. Dans bien des secteurs, un marché intérieur dynamique joue un rôle essentiel comme plateforme de développement de la capacité d'exportation. C'est à l'évidence le modèle suivi dans les télécommunications, l'audiovisuel, la distribution, les services portuaires et connexes et l'enseignement supérieur - et même, dans certains cas, dans les secteurs liés au commerce électronique (ainsi, un exportateur de solutions de commerce électronique a signalé qu'il était difficile de démontrer aux acheteurs étrangers l'efficacité des solutions qu'il avait mises au point dans la mesure où il ne pouvait les tester que sur un marché intérieur étroit). La démarche est généralement la suivante : les entreprises alimentent d'abord le marché intérieur et attendent d'avoir atteint la taille critique pour commencer à exporter dans les pays de la région et/ou dans ceux, comme l'ancien colonisateur, avec lesquels existent des liens historiques forts. Enfin, elles peuvent devenir des acteurs du marché mondial. Les exceptions à cette évolution générale sont les services dépendant des TIC et les services connexes, pour lesquels la situation géographique a moins d'importance, ainsi que le tourisme, secteur qui permet à une société de viser dès le départ le marché mondial.

26. Le schéma « activités intérieures-régionales-mondiales » de développement des échanges connaît une variante, dans les cas où une capacité d'exportation mondiale a été développée par des services importés. Par exemple, si, dans les services financiers, l'étude n'a guère trouvé de cas d'entreprises nationales de services financiers de pays en développement qui exportent des services, elle a permis de constater qu'il existait des filiales d'entreprises de pays développés implantées dans ces pays qui exportaient, tant à destination du pays d'origine de la société mère qu'à destination d'autres pays, développés et en développement ${ }^{10}$.

10 Cette remarque souligne une autre difficulté de l'étude ; à l'époque des sociétés transnationales, dont les filiales et les succursales opèrent dans de nombreux pays, tandis que les fusions et acquisitions représentent une large part des flux d'IDE, parler de la nationalité d'une société ne va pas de soi ; ainsi, le $13^{\text {ème }}$ port le 
27. L'étude fait effectivement ressortir le rôle important des liens entre sociétés dans la constitution d'une capacité d'exportation ; cela vaut en particulier pour les partenariats avec des sociétés de pays développés, mais aussi avec des sociétés d'autres pays en développement. Très peu d'exportateurs sont des entrepreneurs individuels. Une étude récente de l'OCDE sur la libéralisation des échanges de services dans les pays d'Europe du Sud-Est montre que l'un des facteurs qui influent sur les performances $\mathrm{du}$ secteur bancaire est le niveau de participation étrangère, qui peut apporte une amélioration des compétences et des pratiques professionnelles.

28. Les liens régionaux, linguistiques/culturels et historiques (par exemple entre les anciennes colonies et leurs colonisateurs) peuvent aussi jouer un rôle important dans les services aux entreprises. Les entreprises dont les activités débordent le marché intérieur exportent souvent d'abord vers les pays avec lesquels ce type de liens existe, les diasporas se révélant importantes dans des domaines qui vont de la médecine traditionnelle aux services audiovisuels. De fait, des créneaux réels pourraient être exploités dans les cas où les facteurs culturels et géographiques sont déterminants pour la réussite de la commercialisation d'un produit - on peut citer comme exemple le parc à thème agricole créé en Colombie, qui exporte maintenant son concept vers des pays en développement et développés, ainsi que les remèdes traditionnels d'un certain nombre de pays asiatiques et plusieurs initiatives en matière d'écotourisme.

29. Les pays développés ayant des liens historiques et coloniaux avec certains pays en développement peuvent aussi représenter une source de capitaux et de crédibilité pour les exportateurs des pays en développement.

30. Cependant, l'étude révèle aussi que, dans un certain nombre de secteurs, on trouve peu d'exemples d'entreprises de pays en développement qui exportent (par exemple l'énergie, la recherchedéveloppement et les services environnementaux). Cette situation peut être due à l'importance des coûts fixes à supporter pour pénétrer ces secteurs, et à l'existence de quelques très grandes entreprises présentes dans le monde entier. Même dans les secteurs où les pays en développement exportent, l'étude révèle aussi un certain nombre de problèmes communs auxquels leurs exportateurs doivent faire face :

- Manque d'accès au financement pour l'exportation ou le développement de l'entreprise :

- Les exportations de services des pays en développement concernent souvent des domaines où les besoins en capitaux sont relativement faibles, ou offrant la possibilité d'accéder aux capitaux par le biais de partenariats internationaux.

- Du fait du niveau peu élevé de revenu sur le marché intérieur, il est parfois difficile pour les entreprises de se constituer dans leur pays une clientèle qui joue le rôle de plate-forme d'exportation.

- Difficulté d'établir une crédibilité auprès des fournisseurs internationaux, en particulier, mais pas seulement, dans les domaines de haute technologie :

- L'idée de l'infériorité des fournisseurs de services des pays en développement reste bien ancrée dans certains secteurs, y compris sur les marchés intérieurs de ces pays. Cela peut créer un cercle vicieux - ou vertueux; le manque de crédibilité fait baisser le nombre de clients et la réduction de la clientèle réduit la possibilité d'apporter la preuve de sa fiabilité. Il peut aussi arriver, lorsqu'un secteur commence à établir sa réputation dans son pays, que la crédibilité et la reconnaissance qui en résulte se renforcent d'elles-mêmes et conduisent à la

plus actif du monde se trouve au Royaume-Uni (Felixstowe), mais il est possédé et géré par une entreprise de Hong Kong. 
création de réseaux locaux d'exportateurs (c'est le cas pour les TIC en Inde, le traitement des données à la Barbade, les services de santé à Cuba). Les problèmes de crédibilité sont résolus, dans certains cas, par l'établissement de partenariats avec des entreprises internationales.

- Manque d'accès à une infrastructure fiable et peu onéreuse :

- La médiocrité des services de télécommunication a des répercussions sur le potentiel d'exportation de services de sous-traitance ou de post-marché, tandis que l'absence d'accès fiable à l'énergie et au transport augmente les coûts pour tous les secteurs de services.

- La médiocrité de l'infrastructure peut aussi limiter la capacité d'une entreprise de fournir un service fiable et aggraver ainsi le problème de crédibilité auprès des clients (certaines entreprises déclarent ainsi avoir perdu des clients étrangers en raison de retards dans la fourniture de services dus à la lenteur ou au manque de fiabilité des liaisons Internet).

- Manque d'accès à un éventail de réseaux formels et informels et aux facilités institutionnelles nécessaires pour le commerce :

- Ces réseaux peuvent inclure tout ce qui va d'un cadre juridique sain pour les entreprises, à des liens avec d'autres exportateurs ou associations d'exportation ou à des réseaux d'entreprises plus larges.

- Pour les services en particulier, étant donné l'étroitesse des liens et les relations symbiotiques qui existent entre les secteurs de services, des problèmes pourraient se poser pour les entreprises de services du fait de la non-disponiblité de services auxiliaires ou de soutien importants sur le marché intérieur. Une société de conseil située dans un pays en développement a déclaré avoir recours à des entreprises étrangères (souvent concurrentes) pour obtenir des services de soutien importants.

31. Enfin, il convient aussi de noter que, si l'étude a identifié un large éventail d'exportations de services des pays en développement, ces exportations ne représentent pour eux, comme pour certains pays développés, qu'une part relativement peu importante de leurs échanges en comparaison des exportations de produits manufacturés et agricoles. Cependant, les exemples fournis dans cette étude montrent qu'il est encore possible d'accroître les exportations de services des pays en développement. 


\section{SERVICES AUDIOVISUELS ET CULTURELS}

\section{Vue d'ensemble}

32. Selon la Classification sectorielle de l'OMC (W/120), l'audiovisuel fait partie des services de communication et comprend : les services de production et de distribution de films cinématographiques et de bandes vidéo; ceux de projection cinématographique; les services de diffusion radiophonique et télévisuelle ; l'enregistrement sonore et d'autres services. La classification W/120 inclut également les services récréatifs, culturels et sportifs, où figurent les services des agences de presse. La partie suivante, consacrée principalement au cinéma et à la télévision, présente aussi quelques éléments relatifs aux services radiophoniques et à ceux des agences de presse. Les secteurs sont traités par région plutôt que par type de service, compte tenu de la convergence croissante des différents types de technologies et des synergies qui existent entre le cinéma, la télévision et les autres médias.

33. Bien que les marchés mondiaux du cinéma et de la musique soient dominés par quelques grandes compagnies, certains pays en développement sont particulièrement actifs, en particulier dans le domaine de la télévision. Au total, plusieurs points intéressants méritent d'être relevés. Certains pays, qui possédaient auparavant une industrie cinématographique importante (Égypte, Brésil), ont cédé du terrain en ce domaine, mais ont acquis une dimension internationale dans la programmation télévisuelle. D'autres, où le cinéma attire un vaste public, ont accru leur audience à l'étranger, souvent en liaison avec l'existence d'une nombreuse diaspora (Inde). En raison de l'évolution de l'industrie cinématographique, plusieurs pays grands producteurs de films s'intéressent maintenant à la post-production utilisant des technologies de pointe et aux effets visuels. C'est aussi un domaine où certains - particulièrement ceux qui disposent d'une main-d'œuvre formée aux TIC - entrevoient de possibles créneaux. En outre, les pays qui ont leur propre industrie cinématographique se positionnent de plus en plus comme des sites de tournage pour d'autres producteurs, en faisant valoir leurs disponibilités en personnel et en équipes qualifiées.

\section{Asie/Asie du Sud}

34. L'Inde est le premier producteur mondial de films, avec une moyenne annuelle de 800 longs métrages et de 900 courts métrages en 52 langues et dialectes différents. C'est une activité en expansion : en 2001, l'Inde a produit 1013 longs métrages contre 855 en 2000. Outre les films, l'Inde produit 40000 heures de programmes de télévision et 5000 titres musicaux. Ce pays est aussi le troisième plus grand producteur de logiciels de divertissement originaux, avec plus de 40000 heures de programmation en 2001. L'ensemble du secteur audiovisuel et récréatif indien (qui comprend les films, la télévision, le câble, les logiciels de télécommunications, la musique, la radio et les spectacles de divertissement en direct) a vu son chiffre d'affaires passer de 100 milliards d'INR en 2000 à 130 milliards en 2001 ; selon diverses prévisions, il va progresser à un rythme annuel moyen de $25 \%$ ou atteindre 481 milliards d'INR en 2005 [Mukherjee, 2002].

35. Le secteur est aussi un employeur important; il fait travailler directement environ 1 million de personnes et indirectement 4 millions [Mukherjee (2002)]. Chaque jour, plus de 14 millions d'indiens vont au cinéma, bien que les recettes de l'industrie cinématographique nationale ne dépassent pas 3.5 milliards d'USD, c'est à dire à peu près $1 \%$ du total mondial [source : CNUCED]. 
36. Le marché international est la principale source de revenus de cette industrie : les exportations de films ont rapporté 2 milliards d'INR en 1998 (198 titres), 4.5 milliards en 2000 (412 titres) et 5.25 milliards en 2001. Les films indiens sont exportés vers 95 pays environ ; les plus grands marchés sont ceux où vit une nombreuse diaspora indienne (le Moyen-Orient, les États-Unis, le Royaume-Uni et le Canada) ainsi que les pays voisins d'Asie du Sud. Les États-Unis et le Canada représentent environ 30 \% des exportations totales, devant le Royaume-Uni avec $25 \%$.

37. Les droits perçus à l'étranger, qui proviennent en grande partie de la diaspora d'Asie du Sud, pourraient couvrir le coût de réalisation d'un nouveau film. Certaines sociétés de production (Yashraj Productions et Rajshri Productions) ont créé leur propre réseau de distribution à l'étranger, alors que d'autres font appel à des distributeurs internationaux (par exemple Sony classic). Les conglomérats étrangers, tels que Sony et Star TV, achètent des droits aux producteurs indiens. Star TV a acquis ceux de près de 500 films indiens et envisage de lancer une chaîne de films en langue hindi [Source : CNUCED].

38. Ces dernières années, on s'est efforcé de créer des studios dotés d'équipements de grande qualité et d'outils de production sophistiqués : Ramoji Film City dispose à Hyderabad de moyens comparables à ceux des studios d'Hollywood. De plus, les systèmes de post-production - qui comprennent les animations, les images de synthèse et les effets spéciaux - ont connu un développement significatif, notamment dans l'optique de constituer un centre de sous-traitance pour d'autres grandes industries cinématographiques - des sociétés comme SIBAR Media et Entertainment Limited ont déjà créé en Inde des studios utilisant les technologies les plus avancées.

39. En Inde, comme ailleurs, on constate une interpénétration croissance du cinéma et de la télévision. Les mesures de libéralisation prises en 1990 ont permis une prolifération des chaînes de télévision privées ainsi que la pénétration des films et des programmes télévisuels indiens sur le marché mondial. Il existe actuellement plus de 60 chaînes de télévision privées, tandis que 60 millions d'indiens possèdent un poste de télévision, 28 millions d'entre eux étant abonnés au câble. Grâce à sa main-d'œuvre qualifiée, à son savoir-faire technique et à la faiblesse de ses coûts de production, l'Inde est également un exportateur de contenu télévisuel. Le Conseil de promotion des exportations d'électronique et de logiciels a estimé les exportations de contenu télévisuel à 3.5 milliards d'INR en 2001 (soit $20 \%$ des recettes totales). Plusieurs sociétés indiennes productrices de contenu examinent également la possibilité de conclure des accords de co-production avec des firmes internationales bien établies [Mukherjee, 2002].

40. L'État et la profession encouragent activement les exportations en prospectant de plus en plus les marchés non traditionnels - comme le Japon et le Kenya. Parmi les diverses incitations offertes par les pouvoirs publics figurent l'exonération d'impôt sur le revenu des exportations de logiciels pour le cinéma et le remboursement de certains droits de douane. Toutefois, le secteur est confronté à de nombreuses difficultés, parmi lesquelles on peut citer : la pénurie de financements ; l'absence de protection de la propriété intellectuelle ; le coût de l'adaptation aux avancées de la technologie et aux normes de production de cette industrie ; les droits de douane élevés sur les équipements importés ; enfin, les frais d'extérieurs.

Zee TV est la plus grande chaîne de télévision du sous-continent ; elle est maintenant accessible dans plus de 100 pays du monde. Au Royaume-Uni, on estime à quelque 700000 le nombre de téléspectateurs qui la regardent régulièrement (Bentley 1999). Comme la télévision reste un produit de luxe dans cette région du monde, Zee TV s'adresse clairement aux milieux favorisés. C'est une chaîne de divertissement familial, qui diffuse des programmes en hindi, en gujarati et en anglais. La plus grande partie des contenus est produite en Inde, mais certains programmes en langue anglaise le sont au Royaume-Uni. L'émission "les oscars de Bollywood ", diffusée sur cette chaîne, a été suivie par plus de 250 millions de téléspectateurs, dont près d'un million d'habitants du Royaume-Uni [Source: CNUCED]

Nimbus, une société indienne produisant des programmes de télévision, a réalisé, en 2000, 65 millions d'INR de revenus d'exportation grâce à des ventes au Royaume-Uni, dans les Émirats Arabes Unis, en Malaisie, en Afrique du Sud, à l'île Maurice, au Sri Lanka, au Bangladesh, aux États-Unis et à Singapour [Source : Internet]. 
Des producteurs indiens comme Television 18 et New Delhi TV ont conclu des accords de fourniture de programmes avec CNBC et Star TV. Des sociétés indiennes, parmi lesquelles UTV Toons et Crest Communication, exportent des dessins animés en 2 et 3 dimensions dans d'autres pays asiatiques et en occident. Les firmes les plus puissantes d'Hollywood commencent aussi à s'intéresser à ces productions [Source : CNUCED].

"All India Radio" est l'une des plus grandes stations de radio du monde diffusant vers l'étranger, aussi bien sur le plan du nombre d'auditeurs que de la diversité des émissions. Elle est accessible dans environ 100 pays (en Asie, Afrique du nord-ouest et de l'est, Australie, Nouvelle-Zélande, Royaume-Uni et dans le sous-continent indien - mais pas sur le continent américain) et diffuse en 26 langues (16 étrangères et 10 indiennes). Elle exporte également des enregistrements musicaux ainsi que d'autres programmes à destination de quelque 150 pays et d'organismes de diffusion étrangers. AIR organise des programmes de formation avec d'autres radios étrangères (la BBC et Deutsche Welle) et offre des moyens en ce domaine à des chaînes de radio d'autres pays en développement. Elle a passé des accords de co-production avec Deutsche Welle et la Société australienne de diffusion. La diffusion sur Internet a récemment permis à AIR d'étendre ses services aux États-Unis, au Canada et en Afrique du Sud. En 2000, elle a lancé un service de radio numérique par satellite au Moyen-Orient et en Afrique. Étant donné l'importance et la dispersion géographique de la diaspora indienne, marché de choix d'AIR, les perspectives de développement des exportations ou de partenariats avec des stations étrangères paraissent très prometteuses [Source : Mukherjee].

Pentamedia Graphics est au troisième rang mondial des entreprises d'animation par ordinateur, derrière les Studios Disney et Lucas Digital. Alors que son siège social est en Inde, elle a récemment investi aux États-Unis pour se donner la possibilité de travailler à Hollywood. Le secteur mondial de l'animation par ordinateur progresse à un rythme annuel de $20 \%$ (il représente actuellement 25 milliards d'USD) ; l'industrie indienne de l'animation a un chiffre d'affaires de l'ordre de 500 millions d'USD, mais qui augmente de quelque $30 \%$ par an [Source : Mukherjee].

Globekids Digital Ltd, créé en 2000, était le premier studio d'animation du Bangladesh. II puise dans l'énorme potentiel de nouveaux artistes et de programmeurs informatiques de ce pays pour exporter de l'animation par ordinateur vers de nombreux marchés. Au cours des deux dernières années, ce studio ne s'est pas contenté d'alimenter des clients en Amérique du nord et en Europe ; il s'est développé au point de figurer parmi les organisations les plus réputées pour la formation et l'emploi de jeunes talents au Bangladesh [Source: groupe Evian].

41. Hong Kong Chine demeure l'un des principaux exportateurs et producteurs de films, au troisième rang mondial derrière les États-Unis et l'Inde. En 2001, sous l'impulsion d'un assouplissement du contrôle des financements cinématographiques par le secteur privé et d'une augmentation de la demande étrangère, Hong Kong a produit 126 films et réalisé 782 millions de HKD de recettes à l'exportation au titre de bandes vidéo, de disques laser et d'autres disques compact. Cependant, une crise a eu lieu en 2002, et la production s'est limitée à 92 films - 67 de format classique et 25 en vidéo numérique [bulletin d'information du vendredi de la BBC, 3 janvier 2003 : «L'industrie cinématographique de Hong Kong en crise »; lundi 7 janvier 2002 : «Embellie de l'industrie cinématographique de Hong Kong »].

42. Le Fonds de développement du cinéma (doté de 12.82 millions d'USD) a été créé en 1999 pour promouvoir le développement à long terme de ce secteur par les moyens suivants : financement de sessions de formation pour les metteurs en scène, les scénaristes, le personnel de production des films, les cascadeurs et les opérateurs d'effets spéciaux; d'une étude pour analyser divers moyens de résoudre les problèmes de financement des films; parrainage de films choisis pour participer à des festivals cinématographiques étrangers ; financement de projets (15 l'ont été en 2001, ce qui a coûté environ 3.01 millions d'USD). Le Fonds a également lancé le «forum de financement de films Hong Kong Asie », pour susciter des occasions d'investissement et de production en commun de films à Hong Kong et dans des pays asiatiques. En janvier 2001, on a créé les Archives cinématographiques de Hong Kong, également dans le but de promouvoir à l'étranger le cinéma de Hong Kong.

43. Hong Kong aborde également le domaine des effets visuels, en plein développement ; le nombre de sociétés spécialisées dans la production numérique, la post-production, les effets spéciaux et l'animation est en augmentation. Le Fonds de développement du cinéma s'est concerté avec des institutions tertiaires et la profession pour évaluer la demande future d'effets visuels numériques et de personnel d'animation [Source: http://www.hongkong.org]. En outre, Hong Kong fait sa promotion comme site où des équipes étrangères peuvent réaliser des films commerciaux, des programmes de télévision et des publicités. Le 
Bureau des services cinématographiques distribue un annuaire de la production de films et des sociétés opérant dans ce domaine à Hong Kong [Source: Film Services Office, autorité dispensant les licences de télévision et d'activités de divertissement, Hong Kong Chine http://www.fso-tela.gov.hk].

44. L'industrie du cinéma est confrontée à un certain nombre de difficultés : l'absence de protection des droits de la propriété intellectuelle, en particulier sur les rentables marchés d'exportation de la Chine et de l'Asie du Sud-Est; les perspectives d'une concurrence chinoise dans la production de films; les coûts d'adoption des techniques de production et normes les plus récentes, notamment dans le domaine des effets spéciaux ; la pénurie de moyens de financement [Source: "Exit the dragon?" PricewaterhouseCoopers, www.pwcglobal.com]. L'accès au marché chinois se heurte à des problèmes linguistiques (choix entre le cantonais et le mandarin) et au fait que la Chine, qui considère les films de Hong Kong comme étrangers, les contingente [Source: "Hong Kong Film at the End of the Reel?" www.time.com].

45. La Thaïlande est également un nouvel arrivant dans le secteur de l'audiovisuel, qui produit des films, des programmes de télévision et de l'animation. L'État soutient fermement les exportations nationales dans ce domaine en organisant des foires commerciales dans le pays (Salon annuel de l'audiovisuel récréatif à Bangkok - BEST) et à l'étranger (par exemple le festival cinématographique AsiePacifique). Les pouvoirs publics organisent également des réunions entre des groupes de clients étrangers qui s'intéressent aux services de divertissement thaïlandais et les producteurs nationaux 2 ou 3 fois par an ; des missions à l'étranger s'y ajoutent de temps à autre. On encourage la participation d'œuvres thaïlandaises aux festivals cinématographiques internationaux et on organise, dans diverses parties du monde, des festivals du film thaïlandais (par exemple à Londres en 2002 - www.thaifilmfestival.com) [www.thainationalfilm.com]. De plus, la Thaïlande offre des services d'aide à la production aux clients internationaux qui tournent dans le pays. Comme d'autres pays, elle s'intéresse maintenant aux services de post-production, notamment à l'animation et aux effets spéciaux par ordinateur.

Lancée en 1951 comme troupe théâtrale à la radio, Kantana emploie maintenant un millier de professionnels dans le pays et à l'étranger ; la société exerce 20 activités différentes, réparties en trois branches : télévision et diffusion, production et activités connexes. Télévision \& Diffusion produit des émissions de jeux, de débats, de variétés et des comédies. Pour se développer sur le marché international, Kantana a acheté les droits de diffusion de la cinquième chaîne cambodgienne et installé des bureaux de représentation en Indonésie, en Malaisie, au Vietnam et au Cambodge ; elle projette d'en ouvrir d'autres [Source: www.kantana.com].

Kantana Film \& Commercial Production vend des services de production et de post-production au secteur de la télévision et du cinéma ; elle compte parmi ses clients internationaux $20^{\text {th }}$ Century Fox, Warner Brothers et Lucasfilms. En outre, Kantana produit des films avec des sociétés multinationales, des publicités télévisuelles internationales, des vidéos musicales et des documentaires; elle offre également des services d'aide à la production aux firmes internationales qui tournent des films en Thaïlande. La branche des Activités Connexes est prestataire de services complémentaires; le marketing, la musique, l'organisation et la gestion de manifestations commerciales, la distribution, enfin la fourniture d'outils pédagogiques pour un MBA de gestion de l'audiovisuel récréatif [Source: www.kantana.com].

Filmart/le marché international de cinématographie et de télévision de Hong Kong, organisé par le Conseil de développement commercial de Hong Kong, a lieu chaque année depuis 1997. Cette manifestation commerciale est un excellent support régional pour permettre à des acheteurs et vendeurs internationaux d'effectuer des transactions sur des films et des programmes de télévision. Filmart 2002 a attiré 1612 acquéreurs en provenance de 36 pays et régions [Source: http://www.hongkong.org].

La société China Star Entertainment prévoit de produire de 20 à 30 films, pour un budget total compris entre 200 millions de HKD (25.6 millions d'USD) et 300 millions de HKD (38.4 millions d'USD) en 2002. [Source: Xinhua News Agency, 14 janvier 2002]. 
Dans le cadre du 10ème plan quinquennal (2001-05), l'industrie cinématographique chinoise cherche à s'internationaliser davantage. L'État va financer cinq festivals cinématographiques annuels, pour présenter quelque 50 œuvres nationales à des publics étrangers ; par ailleurs, une nouvelle équipe de responsables d'études des marchés étrangers définira les moyens de commercialiser la production du pays sur les marchés étrangers en s'appuyant sur la coopération internationale. Le plan encourage également les investisseurs étrangers à investir pour moderniser les salles chinoises et pour tourner des films en coopération. De 1996 à 2000, les films chinois ont obtenu 82 prix dans 58 festivals internationaux et les ventes sur les marchés étrangers ont représenté 115 millions de CNY (13.86 millions d'USD). [Source : (c) Centre chinois d'information sur Internet, Xinhua 29/03/2001].

\section{Afrique et Moyen-Orient}

46. L'Égypte dominait auparavant le marché cinématographique arabe avec, à son apogée, une production annuelle de l'ordre de 60 à 70 films, qui comprenait des comédies musicales très populaires. Dans les années 1990, la production de films a fléchi de quelque $75 \%$, mais celle d'émissions de télévision est restée forte, surtout à l'intention de la clientèle des États du golfe persique. La « cité de production des médias » (Media Production City) alimente la plus grande partie du monde arabe ; ses feuilletons télévisés et émissions de radio sont vendus à de nombreux pays arabes, dont l'Arabie Saoudite, le Koweït et les Émirats Arabes Unis. En outre, les chaînes satellitaires égyptiennes diffusent au Moyen-Orient, en Afrique, en Europe, en Asie et sur la côte est des États-Unis au moyen de NileSat, le satellite propriété de l'Égypte, qui loue également ses canaux à d'autres stations (par exemple Dubaï 33). La radio égyptienne diffuse quotidiennement à l'étranger en 33 langues [Source : www.newsvote.bbc.co.uk].

\footnotetext{
Media Production City (MPC) est le plus grand complexe médiatique et d'information jamais établi en Égypte conjointement au satellite égyptien "NileSat », il permet à ce pays de rester à l'avant-garde en matière de télévision satellitaire, tout en disposant de moyens de production télévisuelle et cinématographique qui répondent aux exigences de la diffusion en direct à l'époque des télécommunications spatiales. Il poursuit plusieurs objectifs: assurer une production télévisuelle compétitive, conforme aux normes locales et étrangères; disposer d'une capacité de production d'au moins 100 longs métrages par an ; faire du MPC une attraction touristique et l'utiliser comme centre de formation des professionnels nationaux et régionaux. Le MPC comprend 14 studios de haute technologie de dimensions diverses, des aires de tournage en extérieur et des locaux couverts pour la production télévisuelle, qui peuvent aussi être utilisés comme salles de conférence et de festival. On trouve dans ce complexe des équipements de fabrication et de traitement ; un laboratoire pour les films, la correction des couleurs, le montage, l'enregistrement sonore final, le doublage et la mise au point de la copie finale. L'Union de la Radio et de la Télévision, qui est l'organisme de diffusion d'État en Égypte, a investi dans le MPC au même titre que des banques égyptiennes et des actionnaires publics. NileSat et le MPC garantissent que la présence des médias et informations de l'Égypte sera renforcée par l'exploitation d'une production médiatique de grande qualité [Source : http://www.touregypt.net/mpc.htm].

AllAfrica Global Media est le plus grand distributeur électronique de nouvelles et informations africaines du monde. La société se présente volontiers comme « le point d'accès à un public mondial s'intéressant à l'Afrique ainsi qu'à un ensemble de technologies de pointe qui comble une faille significative du marché en matière de transactions sûres, accessibles, peu coûteuses et de gestion des données ». Enregistrée à l'lle Maurice, AllAfrica, qui est présente à Johannesburg, Dakar, Abuja et Washington, fait partie d'un groupe de sociétés qui collecte, traite et distribue des informations en provenance de toute l'Afrique à l'intention de millions d'utilisateurs finaux. Sa branche de services d'information électronique a des centaines de clients internationaux, parmi lesquels des " grossistes" de l'information comme LexisNexis, Reuters (Factiva), Bloomberg et le service d'information du Financial Times. AllAfrica offre aussi des informations d'affaires de première importance et des nouvelles fraîches en continu, captables sur des appareils sans fil comme les téléphones portables et les agendas électroniques. AfricaNet Trust, située à Johannesburg, est une filiale qui produit des articles originaux sur l'Afrique ("Dateline Africa »), pour diffusion sur les médias classiques et sur Internet. La fondation AllAfrica, organisation à but non lucratif, parraine des journalistes africaines et lance des chaînes africaines thématiques consacrées à des problèmes urgents, comme la solution des conflits, la science et la technologie, la santé et la médecine, le développement durable. AllAfrica a pris la suite d'Africa News Service ; cette entreprise à but non lucratif a produit pendant deux décennies des articles et des reportages, qui lui ont valu des prix, à l'intention de médias de premier plan comme National Public Radio, le Washington Post et la BBC; elle a ensuite transféré son activité sur Internet. A la fin de 1999, le conseil d'administration d'Africa News a reconnu que le site ne pouvait continuer à croître de $25 \%$ par trimestre sans investissement. Afin d'assurer sa survie, on a transféré ses archives à AllAfrica, qui a élargi la base de fournisseurs de contenu et développé de nouveaux services [Source: Internet].
} 
Al-Jazira (La péninsule), la célèbre chaîne de télévision satellitaire arabe, s'enorgueillit de donner des informations sur le Moyen-Orient d'un point de vue arabe, tout en s'appuyant sur l'expérience professionnelle de collaborateurs qui ont travaillé dans les médias occidentaux. Elle obtient régulièrement les plus hauts indices de satisfaction des téléspectateurs au Moyen-Orient et en revendique 35 millions. Depuis son lancement, en 1996, ses sources de financement ont été l'émirat du Qatar, les recettes publicitaires, les redevances des téléspectateurs et les accords passés avec d'autres diffuseurs. Elle en a récemment conclu un pour diffuser sur Sky numérique au Royaume-Uni et en Europe. La popularité de cette chaîne découle de sa couverture de l'information et de débats animés sur des questions sensibles d'ordre politique, social et même sexuel. Les scoops journalistiques d'Al-Jazira ont attiré l'attention sur elle [Source: BBC Monitoring].

\section{Amérique latine}

47. Au début du $20^{\text {ème }}$ siècle, la production cinématographique du Brésil atteignait 100 films par an. Elle a connu jusqu'en 1993 des hauts et des bas, imputables aux interventions de l'État, aux tendances du marché et aux crises économiques. La production et la distribution de films ont été stimulées par une libéralisation progressive et par une nouvelle loi sur l'audiovisuel, adoptée en 1993. Il s'en est suivi un développement des infrastructures, dans le cadre d'initiatives communes du secteur public et du privé, appuyées par un dispositif d'incitation. En conséquence, le marché du cinéma et le secteur audiovisuel se situent depuis 1995 entre le $8^{\text {ème }}$ et le $10^{\text {ème }}$ rang mondial; ils réalisent un chiffre d'affaires de 8 à 10 milliards d'USD, c'est-à-dire près de $1 \%$ du PIB [Source : Diagnostico Governamental, Secretaria do Audiovisual, Ministerio do Cultura 2000].

48. La télévision est devenue est un secteur d'importance majeure en Amérique latine, où de grands opérateurs gèrent diverses co-entreprises et d'autres formes de partenariat dans l'ensemble de la région.

Grupo Televisa, qui possède des intérêts dans la télévision, la radio et l'édition, occupe la première place au sein des médias latino-américains. II est en tête au Mexique pour la télédiffusion avec quatre chaînes et plus de 225 stations affiliées (environ 200 appartiennent à la société). Le groupe dispose aussi d'une participation de $51 \%$ dans la coentreprise Cablevisión et de $60 \%$ dans Innova, qui gère le système de télévision satellitaire direct à domicile (dont Televisa contrôle 30 \%).

La branche d'édition de la société, Editorial Televisa, est un important producteur de magazines en langue espagnole. Televisa est également propriétaire de deux équipes de football, d'un stade et de $50 \%$ du capital de 17 radios mexicaines. Bien que Televisa garde la part du lion de la télévision en langue espagnole, d'autres groupes de la région s'associent avec des partenaires étrangers pour augmenter leur part du marché. II s'agit notamment de l'argentin Grupo Clarin, un éditeur de journaux et diffuseur qui pèse 2.2 milliards d'USD, et du département télévision du groupe vénézuélien Cisneros (594 millions d'USD).

Une plate-forme régionale de télévision par satellite appelée Sky Latin America, rassemble Televisa, le groupe News Corp. de Rupert Murdoch, le diffuseur brésilien Organizac Centses Globo et Liberty Media International, d'Englewood. Televisa détient une participation de $60 \%$ dans la division mexicaine de Sky qui, avec 348000 clients à la date du $1^{\text {er }}$ juillet, est la meilleure réussite de la co-entreprise.

Televisa possède aussi des participations minoritaires dans d'autres entreprises et il tire parti de sa tête de pont sur le marché hispanique. Televisa s'est allié avec Venevision, du groupe Cisneros, et un producteur américain pour acheter en 1992 Univision, une entreprise de Los Angeles. En échange de programmes qui ont permis à Univision d'obtenir plus de $80 \%$ des téléspectateurs américains de langue espagnole. Televisa perçoit un pourcentage des recettes. Televisa et Univision, qui ont réalisé l'année dernière un chiffre d'affaires de 577 millions d'USD, envisagent maintenant d'introduire les chaînes payantes de Televisa sur le câble américain.

Les négociations pour la création d'une co-entreprise visant à promouvoir à l'étranger les chanteurs et autres musiciens de Televisa sont bien avancées. De plus, les partenariats avec l'Espagne et la France exporteront en Europe sa formule de feuilletons télévisés. Les telenovelas sont déjà réputées à l'étranger [Source : édition de Newsweek sur Internet].

49. Il existe aussi une importante activité de commercialisation de contenu dans la région et au-delà. Les «telenovelas» sont les programmes latino-américains qui s'exportent le mieux, les principaux 
vendeurs étant Televisa (Mexique), TeleFe (Argentine), Venevision et RCTV (Venezuela) ainsi que TV Globo (Brésil). Ces sociétés écoulent leur production sur le marché international et s'en échangent une partie entre elles. Les gros acquéreurs sont notamment les autres pays latino-américains, l'Espagne, l'Italie, la Russie, Singapour et la Malaisie. Les Telenovelas ont également été adoptées par les chaînes américaines qui diffusent en espagnol, parmi lesquelles Univision, Telemundo et GEMS International. En fait, plusieurs chaînes câblées des États-Unis tirent une grande partie de leur programmation d'Amérique latine [Source: www.channel1.com].

La chaîne GEMS International Television, lancée à Miami en 1992, offre 24 h/24 des programmes en langue espagnole destinés aux téléspectatrices hispaniques. Elle importe environ $75 \%$ de ses programmes d'Amérique latine où elle compte 3.7 millions d'abonnés, à qui s'ajoutent 864000 aux États-Unis. Ses programmes quotidiens comportent deux à trois telenovelas importées, des émissions de jeux, des magazines de variétés et des débats [Source: www.channel1.com].

D'autres chaînes câblées émettant des États-Unis et visant le marché hispanique acquièrent des émissions produites en Amérique latine ou les retransfèrent ; ce sont : Galavision, à vocation de divertissement familial, qui importe surtout des émissions de variétés et des sitcoms ; Canal Sur, qui retransmet quotidiennement des émissions d'information et des spectacles des grandes chaînes sud-américaines à 4.8 millions de ménages d'Amérique latine et des États-Unis ; TeleNoticias, chaîne intégralement dédiée à l'information, qui appartient à Telemundo et est suivie par 20 millions de ménages aux États-Unis; elle propose de courts bulletins d'information qui proviennent du Mexique, d'Argentine et du Venezuela. L'audiovisuel indien occupe aussi une place croissante sur les marchés mondiaux. Les exportations de l'ensemble des produits audiovisuels indiens ont rapidement augmenté au cours de la dernière décennie, à telle enseigne que les ventes de vidéos/films/logiciels ont progressé de $65 \%$ entre 1999-2000 et 2000-2001, passant de 7.25 milliards d'INR à 12 milliards d'INR [Source: www.channel1.com].

50. On estime également que les échanges de services culturels offrent de réelles possibilités en Amérique latine.

Au Brésil, l'Institut de planification culturelle (Instituto Plano Cultural), organise des manifestations culturelles; il propose également des services d'évaluation de parrainage culturel aux sociétés (et à leurs succursales locales) situées dans la région (Argentine, Chili, Uruguay et Paraguay), aux États-Unis, en Europe, en Chine et au Nigeria. Selon Rafael Raddi, un représentant de cette institution, "le Brésil peut offrir un grand nombre d'occasions et de perspectives dans le domaine de l'évaluation du parrainage culturel ». II explique qu'il existe sur le marché une très forte demande pour commercialiser l'image culturelle de l'Amérique latine et en faire usage à l'échelle mondiale, alors que sur place les professionnels sont rares [Source : étude de l'OCDE]. 


\section{SERVICES AUX ENTREPRISES}

\section{Vue d'ensemble}

51. Cette partie traite d'un ensemble de services qui figurent, au sein du classement sectoriel des services de l'OMC (W/120), dans la catégorie des « services fournis aux entreprises ». Elle laisse de côté les services professionnels et la plus grande partie des services informatiques et connexes, qui font l'objet de rubriques distinctes ; ainsi, les entreprises dont les activités sont axées sur le traitement des données sont classées parmi les services informatiques et connexes. Cette partie inclut néanmoins des sociétés offrant divers services aux entreprises, y compris des services de traitement de données, ceux-ci n'étant qu'un élément de l'offre proposée aux clients. De même, certaines des sociétés présentées dans cette partie peuvent commercialiser des services liés aux logiciels dans le cadre d'une prestation globale. On s'est efforcé de distinguer les activités premières des entreprises qui apparaissent dans chaque partie, mais certains chevauchements sont inévitables. La présente partie comprend aussi une sélection d'autres catégories de services fournis aux entreprises - à savoir la recherche-développement, les services immobiliers et d'autres services aux entreprises - en particulier la publicité, les études de marché, le conseil en gestion, le placement et d'autres services techniques secondaires tels que l'impression et la traduction.

52. Considérés dans leur ensemble, les services aux entreprises augmentent d'environ $10 \%$ par an et leur chiffre d'affaires global atteignait en 1999 près de 1500 milliards d'USD. On estime, que dans la seule zone de l'OCDE, ils ont créé des emplois pour au moins 11 millions de personnes [Source: OCDE, 1999]. Plusieurs facteurs sont à l'origine de cette brillante performance d'ensemble : le redéploiement général de l'économie au profit des services, la montée d'une économie fondée sur le savoir, la nécessité d'une plus grande souplesse au sein des entreprises, la spécialisation et la division accrue du travail dans de nombreux domaines, la sous-traitance utilisée par des sociétés réputées, enfin l'évolution vers des unités de production et des firmes de plus petite taille. Les États influent sur la demande de services rendus aux entreprises en encourageant les investissements incorporels du secteur privé, par exemple dans la R-D et l'organisation, et en encourageant l'offre de ces services au moyen de divers organismes intermédiaires.

53. En outre, la convergence de l'informatique et de la baisse du coût des télécommunications internationales a transformé les données en une matière première que l'on peut faire circuler instantanément autour du monde. De plus en plus, le traitement de nombreuses fonctions de services non essentiels, notamment les tâches administratives de routine, la gestion de clientèle et les travaux techniques secondaires se déplace vers des sites où il peut être réalisé avec la plus grande efficacité. La sous-traitance et les activités de post-marché à l'étranger, qui supposent le recours continuel à une base de sous-traitance dans un autre pays, sont des pratiques de plus en plus répandues dans les entreprises de l'OCDE et se rangent désormais parmi les secteurs à plus forte croissance des pays en développement.

54. Compte tenu de l'importance du phénomène de la sous-traitance et du fait que les activités soustraitées comprennent un large éventail de services, classés souvent dans différentes catégories, cette partie aborde d'abord la sous-traitance; elle présente ensuite des exemples d'exportations de services dans des domaines comme le conseil, les études de marché, le recrutement de la main-d'œuvre, les autres services aux entreprises et l'appui technique. 


\section{Services de sous-traitance/post-marché}

55. On assiste à une diversification rapide des services administratifs et de gestion de la clientèle qui sont sous-traités. A la fin de 2001, le chiffre d'affaires mondial des opérations effectuées à l'étranger était estimé à 6.4 milliards d'USD et on prévoit qu'il atteigne 62 milliards d'USD d'ici 2008. Ce secteur devrait connaître une croissance exceptionnelle [Source : Evalueserve]. Une liste des sociétés multinationales qui sous-traitent aux pays en développement figure au tableau A.1 de l'Annexe, Partie I.

56. Grâce aux arbitrages sur les coûts du travail, mais aussi à l'esprit d'entreprise et aux compétences informatiques de leur main-d'œuvre, les pays en développement contrôlent une part de plus en plus large du marché mondial des services, allant des centres d'appels à distance à la conception de logiciels sophistiqués. Il peut en résulter pour les entreprises des économies de l'ordre de 30 à $35 \%$, et jusqu'à $50 \%$ en longue période [Source: Evalueserve]. Les activités habituellement sous-traitées aux pays en développement comprennent l'élaboration de résumés et de répertoires, l'enregistrement et le traitement de données, leurs stockage, l'édition électronique, la transcription juridique, les services annexes de contentieux, la gestion du publi-postage, celle des dossiers médicaux, la transcription médicale, les services de secrétariat à distance, la rédaction technique, le télé-marketing, les télé-services, la conception et la gestion de sites Internet.

57. L'Inde a été à l'avant-garde pour exploiter les possibilités de travaux dans les domaines des technologies de l'information et des processus professionnels secondaires; l'exportation de ce type de services lui a rapporté 10 milliards d'USD et environ 185 des 500 sociétés classées par Fortune ont soustraité une partie de leurs besoins de logiciels à des entreprises indiennes. Une étude comparative d'un institut de recherches privé, Evalueserve, a cité l'Inde comme site le plus attractif de la sous-traitance grâce aux compétences, aux capacités et à l'éthique professionnelle de sa nombreuse main-d'œuvre (2.1 millions de diplômés de l'enseignement supérieur par an et 300000 ayant suivi un troisième cycle). Le faible prix relatif de ses travailleurs hautement qualifiés offre les meilleures possibilités d'arbitrage du facteur travail aux entreprises situées en Europe de l'ouest et aux États-Unis, tant sur le plan des charges salariales que sur celui de la productivité. La privatisation du secteur des télécommunications a permis une baisse de $85 \%$ des prix pendant les trois dernières années et la situation internationale en matière de bande passante a beaucoup progressé.

58. En Amérique latine, le Mexique devient un sous-traitant favori des technologies de l'information et de l'ingénierie pour les sociétés américaines qui préfèrent travailler avec des partenaires géographiquement proches. Au fur et à mesure que les entreprises électroniques et les constructeurs automobiles délocalisent leur production de l'autre côté de la frontière, la demande de techniciens augmente vivement. Des télécommunications bon marché et une main-d'œuvre instruite expliquent également que San José de Costa Rica attire beaucoup de centres d'appel ciblant les consommateurs de langue espagnole aux États-Unis et en Europe [Source : BusinessWeek].

59. A quelques exceptions près, les activités post-marché en Amérique latine restent surtout la chasse gardée des sociétés américaines. Les principaux défis à leur croissance dans cette zone ont été le développement des infrastructures de télécommunications et la déréglementation de ce secteur. Néanmoins, des pays comme l'Argentine, le Brésil, l'Equateur, le Mexique et le Pérou disposent aujourd'hui de téléports qui peuvent être utilisés. La société brésilienne New Work Station Telemarketing est l'un des dix plus grands centres d'appels du monde. Au Costa Rica, la zone commerciale privée Metro fait activement la promotion des investissements en centres d'appels, tandis que l'Uruguay propose aux sociétés étrangères des services de traitement des données. La Colombie et le Venezuela créent actuellement des centres d'excellence au service des sociétés pétrolières et gazières. 
60. Les Philippines, la Pologne, la Hongrie, la Russie, la Jamaïque et la Chine ainsi que d'autres économies en développement et en transition sont en train de pénétrer à leur tour sur le marché. Aux Philippines, plus de 8000 entreprises étrangères sous-traitent à neuf parcs de technologies de l'information reliés par fibre optique. La Roumanie et la Bulgarie deviennent des ateliers de TI des multinationales allemandes, tandis que les fournisseurs américains et indiens de ces services ouvrent des bureaux en Hongrie, Pologne et République tchèque pour utiliser, à l'intention de leurs clients européens, la nombreuse main-d'œuvre germanophone et anglophone. En Russie, qui compte un réservoir disponible de maîtres et docteurs en sciences, TI et mathématiques, les exportateurs de logiciels emploient une dizaine de milliers d'ingénieurs spécialisés dans des projets complexes. Boeing, Motorola et Intel possèdent tous des centres de recherches dans ce pays. La Chine s'affirme actuellement comme un pôle essentiel de développement de produits pour General Electric, Intel, Philips, Microsoft et d'autres multinationales de l'électronique ; son point fort est la conception de matériels et de logiciels intégrés. Des centres d'appels travaillant pour le Japon et la République de Corée s'installent également dans les villes côtières chinoises [Source: BusinessWeek; www.infoworld.com].

61. Les pays des Caraïbes sont eux aussi de gros fournisseurs de services post-marché. Certains, comme la Jamaïque, ont d'abord investi dans les téléports (centres « intermodaux » des réseaux à bande large) pour obtenir des contrats de réservation et de réception dans le secteur du tourisme ainsi que pour compléter les contrats de base de traitement des données. La Barbade et la Jamaïque possèdent maintenant des activités florissantes de post-marché, qui se sont surtout développées en attirant des entreprises « captives » (c'est-à-dire des filiales que détiennent des sociétés afin d'assurer leurs propres engagements). La République dominicaine et Sainte-Lucie sont entrées plus récemment sur le marché, tandis que les économies plus petites de l'est des Caraïbes collaborent de façon croissante pour promouvoir leurs capacités en matière de travaux post-marché.

62. Les autres zones où existent des activités post-marché s'appuyant sur des téléports sont Hong Kong, la République de Corée, la Malaisie, le Pakistan, Singapour et le Taipeh chinois. Au Vietnam, marché à faibles coûts et doté d'une main-d'œuvre qualifiée, ces activités font leurs premiers pas.

Depuis ses débuts, la société indienne Airline Financial Support Services (AFSS) existe sous forme de coentreprise entre Swiss (qui en détient $75.1 \%$ ) et Tata (qui détient $24.9 \%$ ), spécialisée dans les services de comptabilisation des recettes. Elle emploie actuellement 400 personnes environ et sa clientèle comprend les compagnies aériennes Swiss, Air Autriche et Qualiflyer. AFSS assure notamment les fonctions suivantes : comptabilité des recettes (ventes et remboursements, trafic, transactions intercompagnies et cargaisons), traitement des chèques clients et application des prix, gestion des recettes, centre multiservices (pour les ventes, les remboursements et la comptabilité financière des compagnies), guichet électronique d'assistance, renseignements, exploitation de données, gestion des relations avec la clientèle, traçabilité des palettes, services électroniques de navigation et programmes pour clientèle à vols fréquents [Source : Evalueserve]. AFSS a permis au groupe d'économiser 8 millions de CHF par an dans le traitement des données et les activités post-marché.

Indosuez sous-traite certains travaux en Inde depuis dix ans, tandis que des sociétés internationales comme HewlettPackard, Motorola, PSI Datasystems, Texas Instruments, Verifone, Tata Information Systems et Infosys ont toutes établi des antennes à Bangalore. Infosys Bangalore gère des prêts hypothécaires pour le compte d'un établissement financier californien (Greenpoint Mortgage, de Novato, en Californie) [Source : BusinessWeek].

Le laboratoire de recherche d'IBM en Inde, créé en avril 1998, est le huitième laboratoire dans le monde du département de recherche d'IBM. Installé sur le campus de l'Institut indien de technologie, à New Delhi, il emploie actuellement 75 chercheurs, dont la moitié sont titulaires de doctorats dans des disciplines en rapport avec l'informatique et les technologies de l'information. Ses travaux sont orientés dans les domaines suivants : commerce électronique (personnalisation, coupons en ligne, marché en ligne) reconnaissance audiovisuelle de la voix, traduction automatique, gouvernance électronique, infrastructures intelligentes (services collectifs en ligne, travail en réseau), gestion du savoir (exploitation des médias, bio-informatique) [Source : Evalueserve] 
Les Philippines forment chaque année 700000 nouveaux diplômés de l'enseignement supérieur et sont un grand fournisseur de comptables diplômés, de concepteurs de logiciels, d'architectes, de spécialistes de télémarketing et d'infographistes, maîtrisant tous la langue anglaise ; à ce titre, les Philippines sont l'un des principaux prestataires de services de traitement de l'information. Le pays est parvenu à devenir un centre prééminent de services financiers et comptables partagés. Parmi les sociétés qui sous-traitent aux Philippines figurent Caltex, P\&G et Accenture, qui délèguent leurs fonctions de back-office comptable. AIG a également choisi les Philippines pour y effectuer ses activités de back-office dans la zone Asie-Pacifique.

Evalueserve est une firme indienne qui accomplit diverses fonctions professionnelles pour le compte de clients européens et nord-américains, auxquels elle offre un service moins coûteux, de meilleure qualité et plus rapide que ce qu'ils peuvent réaliser eux-mêmes. Les fondateurs, Alok Aggarwal et Marc Vollenweider, ont remarqué que les entreprises des pays de l'OCDE effectuaient de nombreuses tâches coûteuses, certes nécessaires, mais étrangères à leur " principale compétence » ; ils y ont vu une occasion de débouchés. Les tâches en question exigent surtout un personnel intelligent et anglophone, dont l'Inde dispose en abondance à un niveau de salaire très inférieur à celui des pays riches [Sources : étude de l'OCDE et site Internet d'Evalueserve]

Le téléport de Montego Bay est équipé pour offrir des services aussi variés que la communication vocale, les télé et vidéo-conférences, le traitement de données. BS\&T Direct, fournisseur d'accès à Internet situé à la Barbade, a créé un centre d'appels qui répond aux questions de clients relatives aux sites de commerce électronique. La société a profité du coût du travail relativement faible de la région des Caraïbes, alors que le boum de l'économie des États-Unis raréfiait la main-d'œuvre disponible ; elle bénéficie aussi d'une proximité géographique qui permet des horaires de travail similaires [Source: Financial Times].

63. Malgré l'obstacle que représentent encore les infrastructures de télécommunications en Afrique et au Moyen-Orient, on y trouve quelques exemples de sous-traitance. La Tunisie accueille trois entreprises de traitement des données, tandis que d'autres activités de sous-traitance ont lieu dans les zones franches exportatrices de l'Ile Maurice et des Émirats Arabes Unis. La société israélienne Gateway Ventures Ltd pilote le traitement de données au Zimbabwe.

64. L'Afrique du Sud est en train de devenir rapidement une solution de rechange séduisante à l'Inde en matière de sous-traitance ; un personnel instruit, parlant le français, l'anglais et l'allemand, travaille pour un nombre croissant de centres d'appels utilisés surtout par des entreprises européennes. Ce pays connaît actuellement un chômage élevé et offre un bon potentiel d'arbitrage sur les coûts de maind'œuvre (économies de 25 à $30 \%$ au lieu de 35 à $50 \%$ en Inde). En outre, l'Afrique du Sud dispose d'infrastructures bien développées, en particulier dans les télécommunications, et est culturellement proche de l'Europe. La compagnie aérienne nationale allemande Lufthansa y a créé un centre d'appels et des sociétés britanniques travaillent également avec divers prestataires de services situés dans ce pays [Source: Evalueserve].

En mai 1999, la compagnie aérienne Lufthansa a créé au Cap un centre d'appels captifs, Global TeleSales. La vocation de cette filiale de sous-traitance est de relier les divers centres d'appels, de les rendre plus accessibles aux clients et d'assurer à ces derniers un service de 24 heures, 365 jours par an. Global TeleSales est surtout destiné à la clientèle du marché européen. $80 \%$ des appels traités émanent de clients allemands, et les $20 \%$ restants comprennent des appels en surcharge provenant des États-Unis et quelques-uns de clients sud-africains. Le centre s'occupe des prises de réservation et des ventes de billets des compagnies Lufthansa Allemagne et Lufthansa Afrique du Sud ; le nombre mensuel d'appels est de l'ordre de 10 millions [Source: Evalueserve].

Teknosell est une petite entreprise kényane, qui exporte jusqu'à $20 \%$ de ses services en Ouganda et en Tanzanie ; elle travaille aussi pour les antennes locales de multinationales américaines et allemandes. Selon Andrew Otsieno, concepteur de sites et d'information chez Teknosell, les meilleures occasions d'exporter sur le marché international pour des sociétés comme la sienne se situent dans les activités de back-office spécialisées dans l'" Information Design ». A ses yeux, les principaux obstacles à l'internationalisation de ces fonctions par les entrepreneurs de pays en développement sont le manque de connaissance des opérateurs du marché et l'incapacité à les convaincre que ces entrepreneurs peuvent offrir des services évolués, comme la conception d'information et de sites Internet [Source : étude de l'OCDE]. 
E-Business Solutions Ltd est une petite firme kényane qui propose pour l'exportation aux États-Unis, au Royaume-Uni et en Australie des services de back-office et de recherche sur Internet. A l'heure actuelle, les exportations d'E-Business Solutions Ltd représentent moins de $10 \%$ de ses activités. Mais son propriétaire, M.Mugure Mugu, s'attend à les voir augmenter grâce à l'expansion générale de ce secteur et aux stratégies de marketing agressives centrées sur les marchés américains et européens, qui ont déjà donné des résultats positifs. Selon lui, les perspectives d'exportation sont bonnes pour les services aux entreprises et services professionnels sous-traités aux économies en développement. II considère que les principaux obstacles sont le défaut de soutien public, la médiocrité des infrastructures de communications ainsi que l'absence de crédibilité et de confiance, facteurs particulièrement importants du fait de la nature immatérielle des services. Cette entreprise a fait elle-même l'expérience de la perte d'un client situé aux États-Unis au terme d'un mois de collaboration, par la faute de difficultés de communication et de la lenteur des transmissions Internet locales. Elle se souvient également d'avoir manqué l'occasion de fournir des services de centre d'appels vers l'extérieur, en raison de la mauvaise qualité des infrastructures, de coûts élevés et de l'impossibilité d'utiliser la technologie VOIP (Voice Over Internet Protocol) pour des raisons politiques [Source : étude de l'OCDE].

65. Bien que la fracture numérique soit un problème sérieux, beaucoup des pays les moins développés disposent déjà d'une forme d'accès à Internet et certains comptent au moins cinq fournisseurs de services en ligne (par exemple le Bangladesh, Haïti, le Mali et la Tanzanie). L'essor de ces activités de service s'est opéré dans une large mesure en l'absence d'aides spécifiques et souvent sans le soutien des plans nationaux de développement.

\section{Publicité et études de marché}

66. Les services de publicité comprennent la programmation, la création et le placement des annonces, la vente et la location d'espaces médiatiques publicitaires ainsi que des services divers, comme la mise à disposition d'échantillons et de matériel spécialisé. Ce secteur s'est largement ouvert aux échanges commerciaux, grâce surtout aux stratégies des sociétés multinationales pour renforcer l'image de marque internationale de leurs produits. Les restrictions dont il fait l'objet se relâchent progressivement, à l'exception du problème majeur de l'hétérogénéité des règles nationales relatives à la nature des actions de marketing et de publicité autorisées ; il s'agit notamment des contrôles visant à protéger la santé publique, à défendre la décence et à sauvegarder la vie privée (OCDE, 1999).

67. La profession va probablement connaître des bouleversements sous l'influence des progrès de la vente directe aux consommateurs (désintermédiation), souvent au moyen de versions électroniques des catalogues d'achats classiques, et aussi parce que les bases de données électroniques qui contiennent des informations sur les acheteurs et les abonnés permettent une approche commerciale plus personnalisée. Dans un monde des affaires de plus en plus interconnecté, où les leaders du marché évoluent vers un modèle d'activité en ligne, les entreprises de services de moindre dimension sont poussées à les imiter, et même les plus petites peuvent travailler à l'échelle mondiale. Les centres d'affaires régionaux qui occupent une position centrale, comme Hong Kong en Asie et le Kenya en Afrique orientale, peuvent jouir d'avantages solides pour fournir des services de publicité.

Market Test AD est une société bulgare qui offre des services d'études de marché, des données et des rapports relatifs au marché bulgare. Sa clientèle se compose des succursales locales d'entreprises britanniques, grecques, suisses et néerlandaises ou de sociétés étrangères originaires de Serbie, d'Allemagne, de Grèce et du Royaume-Uni qui souhaitent s'implanter sur le marché bulgare. Les entreprises bulgares proposant des services de publicité et d'études de marché trouvent de nombreux débouchés en aidant des clients étrangers désireux de pénétrer sur le marché bulgare sans investir directement dans des activités de publicité et d'étude du marché spécialement conçues pour ce pays [Source : étude de l'OCDE].

Zen Creation, entreprise de Côte-d'ivoire, fournit des services aux entreprises, tels que campagnes de publicité, graphismes, édition et gestion de cadeaux de société. Elle exporte en France et en République de Corée. Toutefois, ses activités d'exportation ne sont ni importantes, ni censées le devenir. L'entreprise estime qu'elles sont surtout entravées par les taxes perçues aux frontières et le coût élevé du transport [Source: étude de l'OCDE]. 
La Société de marketing du Kenya (MSK) a passé un accord de coopération avec l'entreprise londonienne creativebrief
pour promouvoir un nouveau service Internet proposé à l'industrie kenyane de la communication. Le service creativebrief
favorisera le Kenya en créant une plate-forme internationale où les agences et les talents créatifs locaux pourront présenter
leurs références sur le réseau, en particulier à la clientèle multinationale de haut de gamme. Le vice-président de MSK, Paul
Kukubo, a la conviction que « Creativebrief offre aux collaborateurs de MSK et au secteur kenyan de la communication en
général un moyen nouveau et peu coûteux de se faire mieux connaître à l'étranger et de développer leurs affaires avec des
clients internationaux ». Creativebrief est le seul forum Internet de marketing et de communication au monde qui permette
aux clients et aux agences d'entrer directement en contact - sans intermédiaire - ce qui simplifie la présentation des
arguments de vente tout en économisant du temps et de l'argent. Le service met en relation des entreprises clientes du
monde entier avec les talents qui conviennent le mieux à leurs besoins, que ce soient des réseaux d'agences, des
indépendants et des agences de relation publique ou des spécialistes des médias et de la promotion, des sociétés de
production et même des créateurs individuels. Depuis le lancement de la société, en 2002, environ 200 clients
multinationaux sont devenus détenteurs d'un code d'accès au forum, parmi lesquels Diageo, Unilever, British Airways, Ford,
Pfizer, Kodak, Siemens et Warner Brothers. [Source: www.msk.co.ke]

\section{Services de conseil}

68. L'avantage comparatif des pays en développement pour les services de conseil en gestion résulte d'un niveau de coûts qui leur permet de concurrencer leurs homologues des pays développés. Des possibilités d'exportation existent aussi dans les domaines où la connaissance des marchés locaux est indispensable et difficile à acquérir pour les non initiés.

En Bulgarie, l'Association des services de conseil international propose une large gamme de services aux entreprises, qui comprend le conseil pour l'organisation de foires et d'expositions, les études de marché, la gestion des investissements, la formation du personnel et la participation à des projets d'infrastructures ; elle travaille pour une clientèle située en Serbie, en Allemagne et en Autriche [Source : étude de l'OCDE].

La société Conseil en gestion de performances, de Dakar (Sénégal), emploie 40 personnes. Installée à Dakar, Ouagadougou et Paris, elle envisage d'étendre sa présence en Côte-d'ivoire, au Bénin et au Mali ; sa clientèle se situe en Afrique occidentale, à l'lle Maurice, au Rwanda et en France [Source : étude de l'OCDE].

Consultoria Colombiana S.A. est une société localisée à Bogota qui compte 400 salariés ; elle offre des services de soutien aux entreprises et joue le rôle de consultant pour des projets d'infrastructures dans les domaines de l'énergie, des transports, de l'environnement et de l'hygiène. Son offre de services comprend les études de faisabilité, la conception, la gestion et la supervision de projets. Son expertise s'appuie sur un éventail de professionnels maîtrisant des disciplines aussi variées que l'ingénierie, la science économique, l'administration des entreprises, l'informatique, la géomatique et les sciences de l'environnement. Jusqu'à $25 \%$ des services sont surtout commercialisés à des résidents du Guatemala, du Honduras, du Salvador, du Costa Rica, de Panama, de l'Équateur, du Pérou et de la Bolivie ; la société participe également à plusieurs co-entreprises internationales avec des homologues du Canada, de France, des Pays-Bas, d'Allemagne, d'Autriche et des États-Unis. On peut citer comme exemples de services exportés la conception de la route de Saint-Domingue à Cruce Rincon de Molinillos, en République dominicaine, et l'étude du facteur nodal pour le système de production d'électricité de ce pays ; on mentionnera également l'étude des pertes d'énergie pour le compte de la ville de Tucson en Arizona. Henry Sanchez, représentant de la société, s'attend à une augmentation de la partie internationale de ses activités, en raison des perspectives favorables des marchés des pays susmentionnés et de l'importance des efforts de marketing [Source : étude de l'OCDE].

Au Chili, Logimex Limitada, située à Santiago, est une petite entreprise spécialisée dans les services de conseil aux importateurs, exportateurs et investisseurs étrangers, qui réalise quelque $25 \%$ de son chiffre d'affaires avec la clientèle étrangère. Chargée d'organiser toute la logistique de la branche chilienne d'une société étrangère de télécommunications, elle assure également la représentation locale de sociétés originaires des États-Unis, du Canada, de France, du Pays-Bas, de Corée, de Taïwan et de Chine [Source : étude de l'OCDE]. 
Zhejiang World Trade Information \& Services Ltd. Cet organisme, affilié à la Chambre de commerce du Zhejiang, à la Chambre de commerce internationale de Chine et à la division régionale du Conseil chinois pour la promotion du commerce international, est spécialisée dans la prestation de services d'information et de conseil en vue de favoriser la coopération commerciale et économique du Zhejiang avec d'autres pays et régions. Sa vocation est très large : fourniture et propagation aux entreprises en général et aux sociétés d'import/export en Chine et à l'étranger d'informations internationales relatives aux marchés et aux économies ; conseil en matière de commerce et de partenariats, choix d'agents commerciaux ou de distributeurs ; conseil en matière de technologies et d'équipements de pointe destinées à la Chine et en provenance de celle-ci ; études de marché et de faisabilité des projets ; activités d'affaires, parmi lesquelles organisation de séminaires, de rencontres en vue d'échanges commerciaux et d'investissements, d'exposition de catalogues, de visites d'usines, études de marchés préalable à des missions économiques et commerciales à l'étranger ; on peut également citer la formation de délégations commerciales pour préparer le lancement de co-entreprises dans des pays et régions à l'étranger, les promotions commerciales et études de marché ; enfin, les services d'agence commerciale pour des transactions nationales et internationales.

69. Les activités de conseil sont également en train d'acquérir une importance primordiale pour beaucoup de sociétés spécialisées dans les technologies de l'information (TI). Comme les clients sont demandeurs d'une réduction des coûts et d'une augmentation de la qualité, ces sociétés ressentent une pression accrue pour devenir vraiment novatrices en offrant aux entreprises clientes des solutions fondées sur la technologie. Selon un article publié dans le Financial Times, TCS, Wipro, Infosys et d'autres entreprises indiennes pensent que, si elles installent et entretiennent des réseaux de TI pour améliorer l'activité, elles peuvent tout aussi bien fournir des services de conseil généraux sur les solutions technologiques à destination des entreprises. Elles ont une structure de coûts qui leur permet de concurrencer les sociétés internationales de services, dont les frais généraux sont en partie gonflés par de coûteux partenariats. Cette situation a deux conséquences : les entreprises indiennes, dont le bilan n'est pas assez solide pour lancer des offres sur les leaders des TI, ciblent les domaines du conseil correspondant à des créneaux dans lesquels des compétences spécialisées sont nécessaires. Wipro a montré la voie en faisant récemment l'acquisition de la division conseil en énergie et services collectifs de la société American Management Systems. Infosys a étoffé ses équipes de consultants et d'experts spécialisés. Mastek, un concepteur de logiciels situé à Bombay, a pris une orientation différente en montant une coentreprise avec la société Deloitte Consulting [Source : Financial Times].

Mastek est une société internationale de TI installée en Inde, d'une valeur de 60 millions d'USD, qui propose des solutions économiques aux 1000 entreprises classées par la revue Fortune. Fondée en 1982, Mastek a créé sa première filiale à l'étranger en 1990, à Singapour; Mastek USA a suivi en 1993. En l'espace de 5 ans, 4 nouvelles filiales sont apparues en Malaisie, au Royaume-Uni, au Japon et en Allemagne. Dernièrement, Mastek s'est diversifiée dans le conseil en gestion.

\section{Autres services aux entreprises et appui technique}

70. L'existence sur le marché de créneaux pour les services spécialisés et de grande qualité offre nombre d'occasions supplémentaires d'exportations aux pays en développement. 
B.A.G. Consulting Ltd, société unipersonnelle située à la Barbade, s'est spécialisée dans la sécurité en milieu professionnel et les certificats médicaux, la formation spécifique des organisations, les audits de sécurité, les enquêtes en cas d'accident et d'incident ainsi que l'aide aux relations collectives du travail. Elle est en mesure d'offrir une telle variété de services grâce aux accords stratégiques qu'elle a noués avec d'autres sociétés nationales et étrangères, qui lui fournissent des services auxiliaires et d'appui. Bien que cette entreprise ne fonctionne que depuis environ 1 an, plus de la moitié de ses services sont commercialisés à l'étranger, soit à des homologues de la région (Trinité et Tobago, St Kitts et Antigua), soit au Royaume-Uni. Le propriétaire, M. Rocheford, prévoit que l'orientation déjà fortement exportatrice va s'accentuer grâce aux perspectives d'augmentation de la demande régionale et internationale de normes de sécurité et à une application plus rigoureuse. II est très optimiste pour l'avenir. Selon lui, « le niveau de formation permet à B.A.G de concurrencer n'importe quelle entreprise internationale ; néanmoins, en l'absence de services secondaires comme les tests et le soutien analytique, la société doit recourir à des fournisseurs internationaux qui peuvent aussi être des concurrents ». La mobilisation de ressources financières pour l'activité d'exportation de B.A.G a constitué un obstacle majeur. M. Rocheford remarque que " les petites entreprises de la région peinent à obtenir des financements permettant d'acquérir les services complémentaires nécessaires pour travailler avec les sociétés internationales; celles-ci réclament des acomptes, alors que B.A.G. ne peut être réglée avant d'avoir achevé sa tâche » [Source : étude de l'OCDE].

Exel India Pvt. Ltd., filiale à $100 \%$ d'Exel Royaume-Uni, propose des solutions logistiques sur mesure à un large éventail d'industriels et de distributeurs. Pour offrir des services $3 \mathrm{PL}$ (logistique fournie par des tiers) à sa clientèle, qui comprend plusieurs multinationales, la société dispose d'un réseau de 24 bureaux et d'environ 700 salariés. Son catalogue de produits comprend toute la gamme complète des solutions logistiques innovantes : il couvre la chaîne complète d'approvisionnement, allant de la conception et du conseil jusqu'aux services d'expédition, de fret, d'entreposage et de distribution, pour finir avec la gestion intégrée de l'information, celle du commerce électronique et l'appui à celui-ci [Source : Evalueserve].

Friolatina S.A., l'un des plus grands industriels argentins, est spécialiste de la fabrication et de la vente à l'échelle mondiale d'équipements commerciaux et industriels de réfrigération, de chambres froides et de panneaux d'isolation. II assure les services après-vente à une clientèle du continent américain (Bolivie, Chili, Équateur, Porto Rico, Panama, Pérou et États-Unis) et d'Europe (Finlande, Italie et Espagne) [Source : étude de l'OCDE].

Editorial Lord Cochrane SA est une petite société chilienne, qui se livre à l'exportation de services d'imprimerie, imprimerie commerciale, pré-impression et marketing. Elle doit sa compétitivité sur les marchés d'exportation à d'importants investissements dans les technologies de pointe et à sa capacité à être le vendeur unique d'un ensemble de services en ce domaine [Source : étude de l'OCDE]

Les prix compétitifs offerts par les imprimeurs et éditeurs malaisiens et la qualité élevée de leurs produits et services ont séduit des acheteurs de Singapour, du Royaume-Uni, de Thaïlande, de Hong Kong, d'Afrique du Sud, de Brunei, de l'lle Maurice, du Vietnam et de Russie. La profession s'attache constamment à réduire les délais de réalisation et beaucoup de ses membres ont investi dans les technologies de pointe, pour assurer eux-mêmes les services de finition et de reliure. L'engagement des autorités malaisiennes à soutenir les droits de la propriété intellectuelle et de reproduction donne une assurance qui conforte la réputation de ce pays comme centre d'activités d'imprimerie et d'édition. Le secteur se prépare également à exporter des technologies et du savoir-faire dans des opérations spécialisées, telles que la gestion d'imprimerie. Enfin, les éditeurs et imprimeurs malaisiens se fondent sur leurs compétences et leur expertise pour nouer des partenariats intelligents et investir à l'étranger [Source: MATRADE].

Au Togo, un modeste traducteur, M. Akoli Penoukou, constatant que ses affaires périclitaient, s'est mis en quête de moyens de redressement. II a commencé à chercher de nouveaux débouchés. Alors qu'il se trouvait dans une pharmacie, il a remarqué par hasard que de nombreux produits pharmaceutiques étaient importés d'Europe et avaient des étiquettes rédigées en néerlandais, espagnol ou français. En dépit de la taille très réduite de son entreprise, M. Penoukou n'a pas hésité à se lancer dans un énorme travail: prendre contact avec les grandes sociétés pharmaceutiques étrangères et leur offrir de traduire les étiquettes, en utilisant Internet, pour accroître leurs parts de marché. Il est également entré en relation avec des chambres de commerce et des associations professionnelles. Six mois plus tard, il déclare disposer d'une agence de traduction enregistrée officiellement en Allemagne et recevoir des commandes de ce pays. II parachève actuellement la création de nouvelles agences de traduction dans trois autres pays, parmi lesquels les États-Unis. Cette expansion internationale a permis d'embaucher trois nouveaux traducteurs togolais, qui se préparaient tous à aller travailler à l'étranger en raison de la situation économique [Source : Centre du commerce international]. 
Fast Sprint srl est une petite société roumaine qui propose des traductions techniques, commerciales, notariales et officielles en anglais, français et espagnol, ou à partir de ces langues, à des clients situés en Serbie, Turquie, France et Autriche ainsi qu'aux antennes locales de sociétés françaises, autrichiennes et grecques. Comme les entrepreneurs locaux et les investisseurs étrangers sont de plus en plus demandeurs de conseils pour trouver des débouchés dans le commerce international et à l'étranger, l'entreprise s'est diversifiée dans ce nouveau secteur [Source : étude de l'OCDE].

Le Sino-Trust Business Translating Center de Pékin (Chine), fournit des services de traduction dans la plupart des langues d'importance commerciale du monde, ainsi que d'autres services aux visiteurs, notamment des services d'hébergement, d'interprétation et d'installation de bureaux [Source : Sinotrust].

La Société d'aménagement de zones d'activités exportatrices Shanghai Jinqiao, fondée par deux diplômés de l'université de Princeton, pratique la promotion et la gestion immobilières, offre des services de location-vente et de courtage, investit dans les droits d'utilisation des sols, construits des infrastructures municipales, décore, investit et opère dans des entreprises. Les cessions immobilières ont représenté, en $2000,75 \%$ de son chiffre d'affaires, les locations immobilières $16 \%$, les ventes et les locations de foncier respectivement $5 \%$ et $4 \%$. 


\section{SERVICES INFORMATIQUES ET CONNEXES}

\section{Vue d'ensemble}

71. La classification sectorielle des services de l'OMC (W/120) inclut dans la définition des services informatiques et connexes les services de consultation en matière d'installation de matériel informatique, les services de réalisation de logiciels, de traitement de données, de bases de données, d'entretien et de réparation de machines et de matériel de bureau, y compris d'ordinateurs, et les autres services informatiques. Ce chapitre est consacré aux services de logiciels et connexes et aux services de traitement de données; les entreprises répertoriées ici dans la rubrique «traitement de données » exercent cette activité à titre principal (pour autant que les informations disponibles nous permettent d'en juger). Certaines entreprises ayant aussi des activités de traitement de données dans le cadre d'une offre globale de services offerts à leurs clients figurent également dans la partie consacrée aux services sous-traités des «services fournis aux entreprises ». De même, les entreprises choisies pour figurer ci-dessous dans la partie consacrée aux services de logiciels sont souvent elles aussi prestataires d'autres services, mais ont été répertoriées ici parce qu'il est apparu que les services liés aux logiciels constituaient une part significative de leurs activités.

72. Les services informatiques et connexes, ainsi que les services de télécommunications, contribuent à la création d'une infrastructure essentielle à la fourniture internationale de services, à laquelle peuvent participer les pays en développement.

\section{Services de logiciels}

73. Les avancées technologiques des télécommunications et de l'informatique ont permis aux pays en développement dotés d'une main-d'œuvre bien formée et d'un coût compétitif de produire et d'exporter dans le monde entier des services informatiques et connexes. La réussite spectaculaire de l'Inde au cours des 20 dernières années est le cas le plus remarquable et le mieux attesté.

74. A l'origine, le développement rapide de l'industrie indienne des logiciels et sa forte orientation vers l'exportation ont été le fruit d'une stratégie publique datant des années 1980. De 1985 à 1990, les entreprises indiennes de logiciels, confrontées à un marché intérieur très étroit et à des réseaux de télécommunications insuffisants, ont privilégié la prestation de services sur place à des clients étrangers, une activité qui représentait $80 \%$ des revenus d'exportation du secteur. Les spécialistes indiens se sont surtout consacrés à la mise au point de logiciels sur mesure destinés à des applications spécifiques, pour utilisateurs finaux, plutôt qu'à celle de logiciels pour application standardisée. Beaucoup de sociétés indiennes ont concentré leurs efforts sur la conception de sous-systèmes qui font partie du produit final des grands vendeurs d'équipements de télécommunications et de communication de données. Cette pratique répandue leur a permis d'acquérir des compétences en logiciels de systèmes et en segments de logiciels intégrés, qui leur permettent de se diversifier dans l'intégration complète de systèmes et dans le développement de produits [Source : divers articles du Financial Times]. 
75. L'industrie indienne des services de logiciels est désormais prospère. Des entreprises comme Infosys Technologies, Wipro, Satyam Computer Services, MphasiS, BFL Group et Digital GlobalSoft ont fait état d'une progression de 18 à $35 \%$ de leurs bénéfices ; la société financière HSBC Securities and Capital Markets, située à Mumbai, prévoit une croissance des exportations de $30 \%$ en 2003, soit plus de 7 milliards d'USD. Principal débouché extérieur, le marché américain absorbe plus de $60 \%$ de la production indienne totale de logiciels. Le faible coût des produits indiens par rapport à la concurrence a permis au pays de bénéficier des tendances actuelles à la compression des charges et à la sous-traitance de certaines fonctions d'entreprises [Source: Far Eastern Economic Review]. Les échanges entre l'Inde et d'autres pays en développement sont également en expansion; de nombreux partenariats se forment notamment avec des entreprises brésiliennes.

Infosys Technologies Ltd., société fondée en 1981, emploie actuellement 11304 personnes. Le chiffre d'affaires de l'exercice fiscal 2001-2002 est estimé à 26035.9 millions d'INR (environ 546 millions d'USD). Infosys Technologies fournit des services de conseil et de TI à une clientèle mondiale. Son siège social est en Inde, dans la ville de Bangalore, mais elle a des bureaux en Argentine, en Australie, en Belgique, au Canada, en France, en Allemagne, à Hong Kong, en Inde, au Japon, aux Pays-Bas, à Singapour, en Suède, en Suisse, au Royaume-Uni, aux Émirats Arabes Unis et aux États-Unis. Infosys utilise un modèle de livraison global (Global Delivery Model) à faible risque, pour accélérer les procédures avec une grande prévisibilité des délais et des coûts. Ses prestations comprennent le conseil en technologies de l'information, l'intégration de systèmes, la réingénierie, les solutions de commerce électronique, les services extra-territoriaux, le développement d'applications, les solutions bancaires, la conception et la mise au point de logiciels. On estime les recettes d'exportations de l'exercice 2001-2002 à 25524.7 millions d'INR (environ 535 millions d'USD), les principaux débouchés étant l'Inde, les États-Unis, le Japon, le Canada, le Royaume-Uni, l'Allemagne, la Belgique, la Scandinavie et l'Australie [Source: www.indiasoftware.com].

Satyam Computer Services Ltd., fondée en 1987, emploie aujourd'hui 8634 salariés et réalise un chiffre d'affaires estimé, pour l'exercice 2001-2002, à 18030.95 millions d'INR (environ 378 millions d'USD). Satyam possède en Inde, au RoyaumeUni, aux États-Unis, en Europe continentale, au Japon, à Singapour et en Australie des centres de développement qui travaillent pour 260 sociétés internationales, dont quelque 67 font partie des 500 du classement de Fortune. Elle aurait réalisé en 2001-2002 des recettes d'exportation de 17030.78 millions d'INR (environ 357 millions d'USD); ses principaux débouchés extérieurs sont les États-Unis, le Canada, l'Allemagne, le Royaume-Uni, le Danemark, Singapour, l'Australie, la Pologne, la Malaisie, le Japon, la Suède, les Pays-Bas et le Kenya. Cette entreprise offre une grande diversité de prestations, parmi lesquelles des services de télécommunications et des services financiers, ainsi qu'un large éventail de services de logiciels et de TI [Source: www.indiasoftware.com].

Tata Consultancy Services, société de logiciels créée en 1983 et dont le siège social est à Mumbai, compte 19000 employés. C'est la plus grande entreprise internationale indépendante de logiciels et de services en Asie, son chiffre d'affaires 2001-2002 étant estimé à 41870 millions d'INR (environ 878 millions d'USD). Elle a plus de 600 clients dans le monde entier, dont plusieurs figurent dans les 500 sociétés classées par Fortune. Au cours de l'exercice 2001-2002, les exportations, évaluées à 38820 millions d'INR (environ 814 millions d'USD), ont porté sur des services comme le conseil en TI, les solutions de commerce électronique et de multimédias, le développement d'applications, les solutions bancaires, la conception et la mise au point de logiciels. Les marchés d'exportation de la société sont les États-Unis, le Canada, le Royaume-Uni, l'Europe continentale, le Japon, l'Asie de l'ouest, l'Asie du Sud-Est, l'Afrique du Sud, la Malaisie, Singapour, Hong Kong et l'Amérique latine. [Source: www.indiasoftware.com]

Tata Consultancy Services a ouvert un centre en Uruguay, où elle prévoit d'investir 30 millions d'USD et de créer 500 emplois dans les cinq années à venir. Appelé Global Development Centre, celui-ci offrira des services en Uruguay, mais aussi au Chili, en Argentine au Brésil et au Venezuela, ainsi que dans certaines régions des États-Unis. Parmi ses clients figureront la banque BCl, AIG et Eli Lilly. Le centre uruguayen est le neuvième de Tata Consultancy Services, qui en a ouvert d'autres à Budapest, Melbourne, Toronto, Tokyo, Phoenix, Columbus, Guildford et dans le New Jersey. [Source: www.tcs.com]

HCL Technologies Ltd. est l'une des principales entreprises indiennes à offrir des services de TI et d'ingénierie de produits à l'échelle internationale. Établie en 1991, elle emploie actuellement 6463 salariés et a réalisé, au cours de l'exercice annuel 2001-2002, un chiffre d'affaires estimé à 16242.59 millions d'INR (environ 341 millions d'USD). La large gamme de services qu'elle exporte comprend des solutions pour bases de données, des services de télécommunications, l'analyse et la conception de systèmes, des solutions bancaires et de santé, ainsi que la gestion de la chaîne d'approvisionnement. Les revenus d'exportation auraient atteint, en 2001-2002, 13200 millions d'INR (environ 277 millions d'USD), les principaux marchés se situant en Asie-Pacifique, en Europe, au Japon et aux États-Unis. [Source : www.indiasoftware.com] 
APTECH, société de TI qui dispense en Inde des formations à l'informatique, a conclu des accords avec deux entreprises brésiliennes, A-Hand et MASV Conseils en TI, pour gérer des centres de formation à Sao Paolo, Parana, Santa Catarina et Rio Grande do Sul. Une autre société indienne, Prologix Software Solutions Pty Ltd,. a passé un accord avec son homologue brésilienne SupportComm pour la commercialisation de leurs logiciels de télécommunications au Brésil. Enfin, Online Company, entreprise située à Goa, et la société brésilienne Infocon travaillent en commun pour traduire, adapter localement et perfectionner des logiciels destinés à être commercialisés internationalement [Source : Site Internet du gouvernement indien]

76. Les réussites informatiques ne sont pas une exclusivité indienne. D'autres régions du monde, en particulier le Moyen-Orient et l'Amérique latine, ont également vu leurs services logiciels se développer, notamment grâce à des efforts coordonnés destinés à promouvoir l'expansion de ce secteur. En Europe de l'Est, on assiste actuellement au lancement de nouvelles initiatives.

L'Association libanaise de l'industrie des logiciels (ALIL), créée en 2002, a pour vocation d' " améliorer la compétitivité de l'industrie libanaise des logiciels ", en encourageant activement ses membres à créer, promouvoir et faire progresser cette activité dans le pays. L'ALIL recrute, conserve et élargit un ensemble d'adhérents qui représentent la majorité des entreprises libanaises du secteur, chacun se vouant à la constitution d'un secteur dynamique des logiciels au Liban. Dotée du statut d'association et située à Beyrouth, l'ALIL ne dépend pas d'intérêts politiques, religieux ou partisans. Elle a organisé diverses activités en direction des entreprises adhérentes et d'un plus large public, afin de lancer, d'encourager et d'améliorer l'industrie libanaise des logiciels en prenant des initiatives et en instituant des partenariats [Source: http://www.alsilebanon.org; étude de l'OCDE].

La société libanaise BML Istisharat est un fournisseur réputé d'assurances bancaires, de logiciels de programmation des ressources d'entreprises (ERP) et de services de logiciels, dont plus de $60 \%$ du chiffre d'affaires proviennent d'exportations aux États-Unis, en Europe de l'ouest et au Moyen-Orient ; ses produits se distinguent par le bon accueil reçu à l'étranger. BML Istisharat compte plus de 300 clients dans le monde entier, parmi lesquels plusieurs entreprises figurant dans la liste des 500 établie par Fortune, la Banque mondiale (BIRD) et l'Association internationale du transport aérien (AITA). Ses services de gestion sont fournis par une structure d'enregistrement de données équipée pour employer jusqu'à 150 opérateurs. Le personnel permanent, qui comprend une dizaine de personnes, se compose d'ingénieurs de réseau, de professionnels de la formation et de cadres. L'entreprise offre également des solutions de réponse médiatique interactive (Interactive Media Response), des sites Internet utilisant la voix, l'intégration de la téléphonie à l'informatique, la télécopie à la demande et la reconnaissance avancée de la voix [Source: www.istisharat.com].

Rushmore, située au Koweït, propose aux entreprises des solutions clé en main qui incluent logiciels et services. Le commerce électronique, la recherche-développement, la mise au point de sites Internet et les logiciels d'entreprise sur mesure représentent le cœur de son activité. Les services et produits fournis aident la clientèle à résoudre ses problèmes professionnels en recourant aux technologies de l'information, afin de donner à chaque projet et service vendu un caractère flexible et approprié au client, d'assurer des solutions sur mesure et de livrer avec un bon rapport coût/efficacité. En constituant un solide réseau international de partenaires, Rushmore vise à garantir à sa clientèle l'achat de solutions fiables pour résoudre les problèmes professionnels. Cette clientèle se situe presque exclusivement dans les pays de l'OCDE [Source: www.rushmorent.com]

L'Argentine, le Brésil, le Chili, la Colombie, le Costa Rica, Cuba, la République dominicaine, l'Equateur, le Mexique, Panama, le Paraguay, le Pérou, l'Uruguay et le Venezuela produisent et exportent tous des logiciels et des services de logiciels. Ils ont constitué l'Association latino-américaine des entreprises de technologie de l'information (ALETI), qui a vocation à développer et à promouvoir les industries nationales de logiciels. Selon la Chambre uruguayenne des technologies de l'information, qui adhère à l'ALETI, nombre de ses 170 membres exportent leurs produits et leurs services sur des marchés comme la Communauté européenne, l'Amérique latine, l'Extrême-orient, les États-Unis et le Canada. [Source: www.cusoft.org.uy]

A l'heure actuelle, le Costa Rica possède plus de 100 sociétés de logiciels, qui emploient plus d'un millier de professionnels et exportent à des pays d'Amérique latine, des Caraïbes, d'Amérique du nord, d'Asie du Sud-Est et même d'Afrique. En 1997, les exportations totales des six plus grandes entreprises de ce secteur ont dépassé 25 millions d'USD. L'objectif pour 2001 était qu'elles soient supérieures à 200 millions d'USD. Le gouvernement costaricain estime que « les logiciels vont représenter au $21^{\text {ème }}$ siècle, pour ce pays d'Amérique centrale, la même chose que ce qu'a représenté le café pendant plus de deux siècles ». 
Un élément essentiel de la politique du Costa Rica a été la priorité donnée l'éducation. Le pays, qui a fixé des normes exigeantes dans ce domaine, a pris des mesures pour que le système d'enseignement produise des travailleurs et des cadres qualifiés. Au vu du nombre limité d'ingénieurs et de techniciens, le gouvernement a adopté un programme énergique pour faire évoluer le capital de connaissances du pays conformément aux besoins du secteur des hautes technologies. L'Institut national d'apprentissage (INA), entité autonome financée par des fonds publics et privés, et l'Institut technologique du Costa Rica (ITCR), de statut privé, sont les principaux formateurs d'ingénieurs. Les efforts consentis par le Costa Rica pour moderniser son système éducatif ont été soutenus par la Banque inter-américaine de développement et par des investisseurs privés. Afin d'encourager la demande, les droits perçus sur les ordinateurs ont été supprimés dans les années 1980. La chute des prix de l'informatique a encouragé son usage, au point que le Costa Rica a maintenant l'un des taux d'utilisation les plus élevés d'Amérique latine [Source: Creating a development dynamic: final report of the digital opportunity initiative - PNUD, Accenture, Fondation Markle].

La Chambre des producteurs de logiciels du Costa Rica, CaproSOFT, a été fondée en 1997 pour intégrer les entreprises nationales productrices de logiciels au sein d'un bloc stratégique, qui permette de renforcer le secteur et de concevoir des produits innovants et de grande qualité destinés au marché mondial. Elle aide les producteurs de logiciels du pays au moyen d'accords, de formations qualifiantes et d'actions de promotion nécessaires à leur développement général. CaproSOFT assure également la représentation de ses membres auprès d'instances nationales et internationales. Le nombre d'adhérents a augmenté rapidement, passant de 16 entreprises à ses débuts, en 1998, à 60 en 2000 . Une association formée de CaproSOFT, de l'organisme de promotion du commerce extérieur du Costa Rica (PROCOMER) et de la Fondation nationale pour la haute technologie (FUNCENAT) a obtenu le concours financier de la Banque inter-américaine de développement, afin d'exécuter le plan national de soutien à la compétitivité du secteur des logiciels. Ce plan est orienté vers l'amélioration des ressources humaines, l'application systématique des normes de qualité en vigueur dans le monde entier et le renforcement du rôle institutionnel de CaproSOFT en sa qualité d'organisation représentant les intérêts des producteurs de logiciels [Source : www.caprosoft.org].

Latina Ltda est une entreprise chilienne d'une vingtaine de personnes, spécialiste de la conception de réseaux routiers urbains et des logiciels spécifiques à ce domaine. A l'heure actuelle, ses activités internationales sont entièrement centrées sur le Pérou. Mais son représentant, Jaime Valenzuela Scholz, anticipe de nouvelles possibilités d'exportation pour l'entreprise à la suite des efforts de marketing qu'elle a consacrés à la promotion à l'étranger de la dernière version de son logiciel DIVA. [Source : étude de l'OCDE]

Au Chili également, la société TUXPAN, spécialiste de la conception de logiciels et du conseil, dispose d'une solide base de clientèle qui inclut plus de 50 des principales entreprises privées et publiques du pays. Elle prévoit comme prochaine étape de pénétrer sur les marchés internationaux en s'associant avec des producteurs de logiciels aux États-Unis et en Europe. Jusqu'à présent, ses efforts d'internationalisation ont échoué, en raison de l'ignorance des procédures d'exportation de logiciels, des droits de douane, de taux de change défavorables et de capitaux insuffisants pour exporter efficacement ; ces obstacles sont cités par Gonzalo Galleguillos Vega, qui appartient à la société. Selon lui, les meilleures occasions d'exportations se trouvent dans la fourniture, exigeante en main-d'œuvre, de logiciels de base et dans la conception de processus spécifiques, domaines où les producteurs chiliens jouissent d'un avantage comparatif dû au faible coût du travail manuel ; en revanche, les principaux obstacles qu'il cite sont liés à l'absence de procédures administratives de certification (ISO) ou de développement (CMM) [Source : étude de l'OCDE].

Xymbol, la division de services technologiques et d'infrastructures d'AllAfrica Global Media, commercialise la plate-forme technologique de base de la société ; elle offre des solutions flexibles, à usage multiple et inter-opérables, pour la gestion de données, la livraison de contenus, la gestion des flux de travail, la fonctionnalité transactionnelle et la transférabilité entre plates-formes. La clientèle se compose de grandes organisations demandeuses de technologies complexes et sophistiquées, qui ne font pas toujours partie des secteurs s'intéressant à l'Afrique ou des médias [Source: www.allAfrica.com].

L'Association nationale des experts formateurs en informatique, CPI-SA, est une société roumaine de taille moyenne, spécialiste de l'information, de la recherche en technologies de l'information et du traitement de données. Elle a créé dans l'ensemble du pays un réseau de plus de 125 centres de formation, conçu pour disposer d'un système d'accréditation et d'une structure de fonctionnement aussi proches que possible des réseaux internationaux de logiciels les plus connus ; ce réseau exporte maintenant dans d'autres pays d'Europe centrale et orientale et du Moyen-Orient. [Source : étude de l'OCDE] Les sociétés polonaises Prokom et Optimus ont réussi dans le secteur des logiciels et comptent des clients dans toute l'Europe de l'est [Source: Financial Times]. 


\section{Traitement des données}

77. Les services de traitement des données constituent aussi un créneau intéressant sur le plan des exportations pour les pays en développement ${ }^{11}$.

Equidata, SGV et Software Ventures, trois entreprises des Philippines, font partie des plus grandes entreprises de traitement des données à l'échelle mondiale. On peut citer également aux Philippines l'exemple de STA, qui a une clientèle aux États-Unis, Omega Computer Corporation, axée sur le marché européen, et SVIC qui peut traiter 175 millions de caractères par mois.

Jamerican Associates est une entreprise de la Jamaïque spécialisée dans le traitement de données ; elle est installée dans la localité touristique de Montego Bay et compte des clients à New York, Toronto et au Canada en général.

EDM International, est une entreprise de sous-traitance de traitement de données des États-Unis qui a installé une partie de ses activités dans le nord du Mexique, profitant ainsi de l'important vivier de d'opérateurs informatiques qualifiés et d'ingénieurs en logiciel diplômés des nombreux établissements d'enseignement supérieur de la zone d'échanges internationale. L'entreprise emploie plus de 2300 personnes et traite plus de 300 millions de documents par an pour ses clients internationaux. [Source : www.edmi.com/offshore.html] 


\section{SERVICES DE CONSTRUCTION}

78. Les services de construction comprennent les travaux d'entreprises générales de construction et de génie civil, d'installation et de montage, d'achèvement et de finition des bâtiments, de préparation des chantiers de construction, ainsi que les travaux d'entreprises de construction spécialisées, et les services de location de matériel de construction ou de démolition pour bâtiments ou ouvrages de génie civil, avec opérateur. La structure du marché se caractérise par la présence de nombreuses petites entreprises opérant dans des zones géographiques limitées et d'un nombre relativement réduit de grandes unités, qui sont en concurrence sur le marché mondial des projets de grande envergure. La hiérarchie des entreprises du secteur change d'année en année, ce qui indique le caractère très ouvert de ce marché. La fourniture des services de construction implique d'abord une présence commerciale, qui ne dépasse généralement pas la durée du projet. Les co-entreprises entre intervenants nationaux et étrangers sont très répandues; elles répondent à des motifs de financement, de transfert de technologies et de savoir-faire ou de réglementation locale.

79. On estime le chiffre d'affaires mondial du secteur à 3200 milliards d'USD par an, sachant que les travaux de réparation et d'entretien représentent le plus gros de l'activité dans les pays développés. Au cours des deux dernières décennies, $70 \%$ de l'activité internationale du BTP, mesurée par le nombre et le montant des contrats, ont été entrepris dans les pays en développement. Les services de construction sont une activité fondamentale dans ces pays, au point de constituer souvent le secteur le plus important de l'économie. Le BTP est aussi un service essentiel d'infrastructures pour toutes les autres branches d'activité et un outil pour améliorer les conditions de vie interne. Certains pays, tels que le Lesotho, s'en sont servis pour faire reculer la pauvreté en milieu rural et le chômage des femmes. En effet, ce secteur, en raison de gros besoins de main-d'œuvre, est un employeur relativement important, qui assure en moyenne $10 \%$ de l'emploi total. [CNUCED, 1999]

80. L'activité internationale des entreprises de construction des pays en développement a surtout commencé à prendre de l'ampleur après l'explosion des prix du pétrole en 1975, quand le Moyen-Orient qui n'avait pas de problèmes de financement - est devenu le principal marché régional en ce domaine. Quelques firmes originaires d'une poignée de pays de la région ont saisi l'occasion offerte par ces débouchés lucratifs; elles ont ciblé des projets d'infrastructures et leur ont assigné du personnel d'étude et des ouvriers de leur propre pays ainsi que de la main-d'œuvre à faible coût venant d'autres nations en développement. Au début des années 1980, leur part du marché international a approché son maximum (20\%) [CNUCED, 1999].

KAZEMA Engineering Projects est une entreprise koweïtienne fondée en 1976 et constituée en société en 1977. C'est une organisation intégrée qui commercialise des produits, des projets et des services au Koweït et dans les pays du Conseil de coopération du golfe persique. Elle est parvenue, au terme d'une croissance régulière, à occuper sa position actuelle au premier rang des entreprises du pays. Ses activités commerciales s'exercent sur une large gamme de marchés : États et institutions publiques, secteurs pétrolier et industriel, commerce et immobilier résidentiel. KAZEMA Engineering Projects a noué des partenariats à l'échelle mondiale avec des industriels, des fournisseurs et des entrepreneurs situés aux États-Unis, en Europe, au Japon, en Extrême-orient, au Moyen-orient et dans les pays du golfe persique [Source : étude de l'OCDE]

Osman A. Osman \& Co., l'un des plus gros entrepreneurs de BTP du Moyen-Orient, a vu son chiffre d'affaires augmenter au rythme de 1 milliard d'USD par an. Après avoir diversifié ses services et ses marchés, il possède actuellement 30 antennes en Asie, Afrique et Europe [CNUCED, 2000] 
81. Plus récemment, de nouvelles entreprises de Chine, de la République de Corée, du Brésil, de Singapour, d'Indonésie, d'Argentine, du Mexique et des Philippines on fait leur apparition parmi les 250 plus grands entrepreneurs mondiaux. Désormais les entreprises de pays en développement font également partie des principaux exportateurs de services de construction. Au sein des 150 plus grandes sociétés de construction classées en 2002 sur la base du chiffre d'affaires réalisé hors de leur marché national, 51 étaient situées dans ces pays ; l'une d'entre elles (la société coréenne Hyundai Engineering and Construction) figurait dans les 20 premières. Parmi ces 51 entreprises leaders de pays en développement, 24 étaient originaires de la Chine, six de Turquie, et cinq de Corée; le Brésil, Taïwan et le Liban comptaient tous deux firmes parmi ces 51. Les autres pays dont une entreprise fait partie des 150 plus grandes sont : la Bulgarie ; l’Égypte ; Hong Kong ; la République de Macédoine ; l'Inde ; Israël ; l'Afrique du Sud et les Émirats Arabes Unis (voir tableau A.2 de l'Annexe, partie I).

La Chine est devenue un exportateur d'ingénierie, de main-d'œuvre et de services de conseil pour la conception de projets. A la fin de la décennie 1970, les activités internationales des entrepreneurs chinois se limitaient à un petit nombre de pays du Moyen-Orient et d'Afrique du nord. Elles s'exercent aujourd'hui dans plus de 180 pays. En 2000 , on trouvait 34 sociétés chinoises parmi les 225 plus grands entrepreneurs internationaux et 5 bureaux d'études chinois étaient classés parmi les 200 principaux acteurs internationaux de ce secteur.

A l'origine, les entreprises chinoises travaillaient surtout dans le bâtiment et la construction de routes, mais, depuis la fin des années 1990, elles lancent des projets à grande échelle dans des domaines plus larges, notamment l'édification d'infrastructures civiles, l'énergie, les télécommunications et la restauration de l'environnement. Le dixième « plan quinquennal » chinois fixe les objectifs stratégiques suivants : en 2005, la Chine devra avoir passé des contrats d'une valeur de 28 à 33 milliards d'USD et devra réaliser un chiffre d'affaires de 22 à 25 milliards d'USD; ces deux grandeurs sont donc censées augmenter de 2001 à 2005 à un taux annuel de 14 à $17 \%$. Au cours de la même période, le nombre de chinois travaillant à l'étranger progressera de $6 \%$ par an pour atteindre entre 550000 et 600000 en 2005.

De 1982 à 2000, le marché asiatique a représenté environ $60 \%$ de la valeur des contrats d'exportation. L'Afrique est un autre marché important, à l'origine de 2.29 milliards d'USD de contrats et de 1.29 milliard d'USD de chiffre d'affaires en 2000. A eux deux, les marchés asiatique $(72.2 \%)$ et africain (13.8\%) représentent $86 \%$ des exportations chinoises, mesurées à la fois sur la base du montant des contrats et du chiffre d'affaires, tandis que les marchés américain $(6.4 \%)$, européen $(5.8 \%)$ et océanien $(1.8 \%)$ contribuent au solde $(14 \%)$. Toutefois, les firmes chinoises ont effectué une percée sur le marché européen où, en 2000, l'encours des contrats a augmenté de $19.4 \%$ et le chiffre d'affaires de $77.2 \%$. Leur croissance a été faible sur le marché latino-américain, où le montant annuel des contrats a progressé de $3.6 \%$. Actuellement, les entrepreneurs chinois contrôlent environ $4 \%$ du marché mondial, si l'on se réfère au chiffre d'affaires.

La plupart des entrepreneurs chinois sont, sous une forme ou une autre, propriété de l'État ou d'une collectivité. La faiblesse des coûts de main-d'œuvre leur a donné un important avantage comparatif, mais ils ont été presque multipliés par 20 depuis le début des années 1980. Les entreprises chinoises ont signalé que leur croissance était entravée par les obstacles suivants : lenteur de la réforme des réglementations publiques; faiblesse des associations professionnelles en comparaison avec la situation des pays développés; petite taille des entreprises ; manque de compétence pour appliquer les technologies avancées; expertise insuffisante des contrats internationaux; inadéquation des capacités de financement; déficience des ressources humaines ; absence de techniques modernes et de pratique de la gestion de projets. En outre, l'octroi de licences d'exportation supplémentaires à des sociétés chinoises (2000 licences à la fin de 2001) a suscité une concurrence féroce, certaines faisant valoir qu'il a entraîné une telle baisse de prix pour les lauréats des appels d'offres que ces derniers ne peuvent faire de bénéfices. [Source: Chen et Sherif]

82. Le faible coût de la main-d'œuvre qualifiée et non qualifiée du secteur de la construction n'est plus le seul atout concurrentiel des entreprises de pays en développement. Leur force d'attraction résulte aussi d'améliorations de la gestion et de l'organisation, et de leur capacité accrue à produire des professionnels dotés de compétences spécifiques: ingénieurs, architectes, concepteurs de projets, économistes, spécialistes des TI, analystes financiers et experts écologiques. De nombreuses entreprises asiatiques et latino-américaines sont aujourd'hui en mesure d'offrir, après la construction, tout un éventail 
d'opérations de gestion et de maintenance, grâce à des logiciels spécialisés ; par ailleurs, les réseaux d'information et de télécommunications permettent aux entrepreneurs de tout pays en développement relié à Internet de prendre part à la concurrence de façon plus systématique, même sur des marchés lointains.

83. Dans les économies industrielles avancées, l'évolution rapide du contexte des affaires, la privatisation des services collectifs et l'intensification de la concurrence nationale et internationale ont poussé les entreprises à adopter de nouvelles stratégies ainsi qu'à se réorienter vers une internationalisation plus marquée de la production. Elles expérimentent notamment de nouvelles modalités de production et techniques de commercialisation pour répartir les risques sur différents segments du marché et/ou pays, mais aussi pour comprimer les coûts internes de production et les dépenses d'investissement consacrées aux nouvelles technologies. La sous-traitance, l'externalisation des services intermédiaires et la formation d'alliances stratégiques ont été les moyens choisis pour atteindre ces objectifs.

84. Les pays en développement ont bénéficié du changement de stratégie des entreprises du monde développé. La sous-traitance permet à leurs PME de jouer un rôle sur des créneaux spécifiques du marché. Ainsi, Bechtel (États-Unis), l'un des principaux entrepreneurs de BTP du monde, a décidé d'installer des centres de production à faible coût en Arabie saoudite et en Inde, qui emploient des professionnels d'origine locale ou de pays tiers. Des accords de coopération technologique ont permis de diminuer les coûts d'organisation et de transaction, tout en facilitant la sous-traitance technique internationale et en contribuant à améliorer les pratiques commerciales et les modes de gestion.

Bechtel Inde a été créée en 1994, sous la forme d'une filiale contrôlée à $100 \%$ par le groupe Bechtel, pour fournir des services d'études, d'achat, de construction et d'appui. Elle emploie environ 400 personnes, qui travaillent sur les projets de Bechtel en Inde et dans le reste du monde. Cette société a collaboré, en matière d'études et de construction, à divers projets de Bechtel, dont la centrale électrique de Quezon (Philippines), celle de Gazlan (Arabie Saoudite), le projet énergétique d'Emcali (Colombie), le projet pétrochimique Ras-Tanura (Arabie Saoudite), la fabrique de composés aromatiques d'Ibn Rushd (Arabie Saoudite), le projet énergétique de Samalayuca II (Mexique), celui de paraxylène pour Chevron (États-Unis), l'agrandissement de l'aéroport international de Dubaï (Émirats Arabes Unis), le projet énergétique de Rocksavage (Royaume-Uni) et celui de Spalding (Royaume-Uni) [Source: Evalueserve].

Orascom Construction Industries $(\mathrm{OCI})$ est l'une des plus grandes entreprises de BTP et de matériaux de construction d'Égypte ; elle offre à des clients publics et privés des services de conception de projets, d'achat et de construction. $\mathrm{OCl}$ a noué des alliances pour la réalisation de projets spécifiques avec diverses sociétés internationales du même secteur opérant en Égypte, parmi lesquelles Bechtel, Consolidated Contractors, Besix, Morrison-Knudsen et Krupp. On a également assisté à une croissance des échanges entre pays en développement, notamment sous forme d'investissements en commun ou d'autres formes de coopération entre exportateurs et importateurs. Les coentreprises, les procédures d'offres conjointes et la coopération inter-entreprises pour mobiliser des ressources financières internationales au service du financement de projets on fait leur apparition.

85. L'édification du pont malaisien de Penang, le plus long d'Asie (13.5 km), a été rendue possible par un accord de coopération avec des partenaires sud-coréens. Pour leur part, les entreprises malaisiennes de construction ont lancé des projets dans des pays en développement d'Afrique et d'Asie, parmi lesquels l'Inde, la Chine, le Vietnam, le Chili, l'Argentine, le Myanmar, le Cambodge, les Philippines, le Soudan et la Namibie. Il s'est agi de routes et d'autoroutes, de logements, d'hôtels, de barrages, d'usines et de centrales électriques [Source: MATRADE Société de développement du commerce extérieur malaisien]. Dans la même veine, une co-entreprise indo-malaisienne a permis à un personnel technique malaisien de se former en Inde à la construction de fabriques textiles modernes [Source : Far Eastern Economic Review]. A la fin de 1999, le Brésil avait 193 projets d'investissements en cours en Chine ; les principaux étaient l'offre de conseils pour l'édification du barrage hydroélectrique en terre du pont de Tiansheng, un projet de construction de routes et la soumission à l'appel d'offres pour la deuxième étape de la construction de l'ascenseur de bateaux permanent du barrage des trois Gorges [Source : Site Internet du gouvernement chinois]. 


\section{SERVICES DE DISTRIBUTION}

\section{Vue d'ensemble}

86. Seuls les opérateurs de taille relativement importante semblent avoir étendu leur activités à l'étranger, sans doute en raison de considérations commerciales et des restrictions qui pèsent sur les échanges. La liste des 200 plus grands distributeurs mondiaux comporte peu d'entreprises des pays en développement, et ce sont celles des États-Unis, d'Allemagne, de France, du Royaume-Uni et du Japon qui dominent. Il faut cependant noter qu'une grande partie des distributeurs de ces pays travaillent aussi pour l'essentiel, voire exclusivement, sur le marché national. Un peu moins de la moitié de ces 200 grands distributeurs se limite au marché national (90 sur 200), et la majorité des 50 premiers distributeurs ont des activités dans plusieurs pays.

87. Même s'ils ne sont pas encore exportateurs, plusieurs entreprises de vente en gros et au détail de pays en développement occupent aussi une place importante sur le marché national, en particulier face à la concurrence des entreprises des grands pays développés. Parmi les exemples de réussite figurent le Groupe Wumart de Pékin, Matahari Putra Prima Tbk, en Indonésie, l'entreprise chilienne Mall Plaza et la société mexicaine Soriana and Controladora Comercial Mexicana.

88. Parmi les neuf entreprises de pays en développement figurant parmi les 200 plus grands distributeurs, quatre sont des entreprises mexicaines (Wal-Mart Mexique se trouve à la $58^{\text {ème }}$ position, Controladora Comercial Mexicana à la $140^{\text {ème, }}$ Grupo Gigante à la $152^{\text {ème, }}$ et Soriana à la $156^{\text {ème }}$ ) mais toutes sauf une (Grupo Gigante) ne vendent que sur le marché mexicain. De même, la seule entreprise brésilienne de la liste (Pao de Acucar) ne fonctionne que sur le marché national. Deux entreprises coréennes poursuivent leurs activités à la fois sur le marché coréen et sur le marché chinois; elles se trouvent au $94^{\text {ème }}$ rang (Lotte Shopping) et au $133^{\text {ème }}$ (Shinsegae). D'autres entreprises de pays en développement occupent la $101^{\text {ème }}$ place (Dairy Farm International, Hong Kong Chine) et la $109^{\text {ème }}$ (Pepkor, Afrique du Sud). [Deloitte Touche Tohmatsu, 2003].

89. Même lorsqu'ils n'exportent pas de services de distribution, ces distributeurs peuvent promouvoir l'exportation d'autres services et produits. Ainsi, Companhia Brasiliera de Distribuicao (CBD), la plus grande entreprise de distribution du Brésil, possède les chaînes de supermarché Pao de Acucar et Barateiro, ainsi que les magasins d'électronique et d'appareils ménagers Eletro et des sites de commerce électronique. La division des exportations de Pao de Acucar cherche à utiliser le pouvoir d'achat du groupe pour amener les produits alimentaires brésiliens sur les marchés internationaux. CBD est pour Casino (qui détient $24 \%$ de son capital) une plate-forme d'exportation vers l'Argentine, l'Uruguay, le Venezuela, la Colombie et la France. La division des exportations a aussi conclu un arrangement pour approvisionner une chaîne de supermarchés de Montréal et cherche à exporter vers l'Australie et l'Afrique. [Source: http://biz.yahoo.com] 
Grupo Gigante (Mexique) regroupe des magasins à bas prix, des services alimentaires, des hypermarchés, des magasins spécialisés, des supermarchés et des entrepôts, le chiffre d'affaires de ses points de vente en 2001 étant estimé à 3243 millions d'USD. Le groupe est présent aux États-Unis et au Mexique [Deloitte Touch Tohmatsu, 2003].

Le distributeur chilien Cencosud devrait racheter la chaîne de supermarchés argentine Disco (qui possède environ 237 magasins en Argentine) au distributeur néerlandais Ahold à la fin de 2003, après avoir acquis auprès de lui la chaîne de supermarchés chilienne Santa Isabel au début de l'année [Source : http:biz.yahoo.com].

Mall Plaza, l'un des principaux opérateurs de centres commerciaux du Chili, a pénétré le marché des États-Unis en ouvrant un établissement à Los Angeles en mars 2003. [Source : www.mallplaza.cl].

\section{Afrique}

Le groupe Shoprite, qui est le premier distributeur de produits alimentaires d'Afrique, possède 645 points de vente en Afrique. Le siège de la société se trouve en Afrique du Sud dans la province du Cap occidental, et le groupe est actuellement présent dans 15 pays d'Afrique (Angola, Ghana, Égypte, Maurice, Madagascar, Ouganda, Zambie, Tanzanie, Mozambique, Zimbabwe, Namibie, Lesotho, Swaziland, Botswana et Malawi) ; il prévoit d'ouvrir un établissement à Mumbai (Inde) fin 2003. Shoprite a adopté cette stratégie d'expansion au-delà des frontières de l'Afrique du Sud neuf ans plus tôt, et la considère comme une évolution essentielle. Ce programme d'expansion en Afrique a aussi abouti à la création d'un important marché à l'exportation pour les producteurs sud-africains [Source: http://www.shoprite.co.za/]

D'abord dirigée par l'entreprise italienne Spa, Uchumi s'est dotée en 1989 d'une direction kenyane. Uchumi est la plus importante chaîne de supermarchés du Kenya, avec un réseau de 15 succursales situées en majorité à Nairobi. En 2002 l'entreprise a ouvert à Kampala (Ouganda) son $27^{\text {ème }}$ point de vente [Source : AllAfrica]

Pepkor (Afrique du Sud) possède des grands magasins, des magasins à bas prix, des magasins spécialisés et des supermarchés, et a enregistré en 2001 un chiffre d'affaires de 4601 millions d'USD. L'entreprise est présente non seulement sur le marché national mais aussi en Australie, au Ghana, au Malawi, au Mozambique, au Royaume-Uni et en Zambie [Deloitte Touch Tohmatsu, 2003].

Le groupe Pick 'n Pay est depuis une trentaine d'années l'un des distributeurs de produits alimentaires, de vêtements et de produits usuels les plus grands et les plus prospères d'Afrique. Fondé en 1967 et introduit en bourse (à la JSE Securities Exchange d'Afrique du Sud) en 1968 (sous le nom de Pick 'n Pay Stores Limited), le groupe a pénétré le marché australien en 1985. II possède maintenant en Australie 74 magasins acquis auprès des chaînes Franklin's et Fresco. Tous ces magasins portent l'enseigne Franklin. Le groupe a d'autres activités comme Go Banking, division chargée de rechercher de nouvelles possibilités d'investissement pour le groupe en Afrique du Sud et ailleurs [Source : http://www.pnp.co.za/]

\section{Asie}

Lotte Shopping (Corée) gère des épiceries, des grands magasins et des supermarchés en Corée et en Chine. L'entreprise a annoncé un chiffre d'affaires de 5400 millions d'USD environ en 2001 [Deloitte Touch Tohmatsu, 2003].

Dairy Farm International (Hong Kong Chine) possède divers magasins - épiceries, magasins à bas prix, drogueries, hypermarchés, magasins spécialisés et supermarchés - pour un chiffre d'affaires de 4877 millions d'USD en 2001. En plus du marché national, l'entreprise est présente en Australie, en Chine, en Inde, en Indonésie, en Malaisie, en Nouvelle-Zélande, à Singapour et au Taipeh chinois [Deloitte Touch Tohmatsu, 2003].

AS Watson \& Co a commencé ses activités à Guangzhou (Chine) en 1828 les a étendues à Hong Kong Chine en 1841. AS Watson Group possède maintenant plus de 3000 magasins et emploie plus de 50000 personnes dans 20 pays d'Asie et d'Europe. Ses trois grands secteurs d'activité sont les produits alimentaires, les produits non alimentaires et les boissons. La vente au détail de produits alimentaires concerne l'enseigne PARKnSHOP qui comporte 250 magasins à Hong Kong Chine et en Asie ainsi que la plus grande chaîne de magasins sépcialisés en vin de Hong Kong Chine, qui vient de s'implanter en Europe par l'acquisition d'un grossiste suisse. La division des produits non alimentaires comprend la chaîne de magasins de produits de beauté et de parapharmacie Watsons Your Personal Store, qui dispose de 650 points de vente dans la région Asie-Pacifique, Nuance-Watson, important exploitant de magasins en franchise à Hong Kong Chine et Singapour; Fortress, qui possède 40 points de vente d'appareils électriques à Hong Kong Chine et dans le reste de l'Asie; et Savers, qui exploite 280 magasins à bas prix au Royaume-Uni. En outre, le groupe a acquis récemment Kruidvat, chaîne comportant 1900 drogueries et parfumeries répartis dans six pays. 
Lianhua Supermarket Holdings Co., l'un des plus grands distributeurs chinois, établi à Shanghai, s'apprête à installer sa première filiale étrangère en Belgique, dans le cadre d'une stratégie d'ouverture de magasins en Europe. Lianhua pense profiter de la facilité d'accès aux produits chinois dont il dispose pour créer une entreprise de distribution en Belgique, spécialisée dans l'exportation de produits alimentaires. Créée en 1991, Lianhua détient plus de 2000 magasins en Chine et a enregistré un chiffre d'affaires de plus de 2.1 milliards d'USD en 2002. [Source: www1.chinadaily.com.cn]

Convenience Retail Asia Limited, membre du groupe de distribution Li \& Fung, a le droit exclusif d'utiliser la marque Circle K pour la vente de produits usuels à Hong Kong, Macau et en Chine.

NTUC Fairprice est la première chaîne de supermarchés de Singapour, avec un chiffre d'affaires annuel de plus de 1 milliards de SGD et une part de marché supérieure à $50 \%$. Après s'être retirée de Malaisie il y a quatre ans, l'entreprise envisage de nouveau d'étendre ses activités à l'étranger, en ouvrant des magasins en Chine et en Inde. Ses activités dans ces pays seront gérées en partenariat avec un distributeur local et une grande entreprise internationale. [Source: www.dbs.com.sg ; www.siamfuture.com] 


\section{SERVICES D'ENSEIGNEMENT SUPÉRIEUR ET DE FORMATION}

\section{Vue d'ensemble}

90. Près de deux millions d'étudiants dans le monde entier reçoivent un enseignement officiel en dehors de leur propre pays. On estime que le marché international des services d'enseignement va tripler au cours des vingt prochaines années. Cela dépasse de beaucoup les taux de croissance observés au cours des vingt années précédentes, période pendant laquelle il a doublé.

91. Cet essor résulte d'un ensemble de facteurs, notamment l'augmentation de la demande de compétences linguistiques et de connaissance des autres pays, au fur et à mesure que se développe une « économie du savoir ». Selon les calculs du gouvernement malaisien, la main-d'œuvre dotée d'un savoir a progressé de $16 \%$ en moyenne depuis 1995. Ceux qui ont été faits pour les pays de l'OCDE montrent que près de $30 \%$ de la population active des économies avancées est hautement qualifiée et occupe des emplois exigeant beaucoup de connaissances. On estime à 30 milliards d'USD le marché mondial des étudiants étrangers, ce qui représente à peu près $3 \%$ des échanges de services au sein des pays de l'OCDE. [Source : www.sitrends.org]

92. Le mode 2, c'est-à-dire la consommation à l'étranger (personnes allant étudier dans un autre pays), est actuellement le plus fréquemment utilisé pour la vente de services d'enseignement, précédant la présence commerciale selon le mode 3 (exemple des universités qui s'installent sur les campus d'autres pays). Toutefois, les nouvelles technologies de l'information sont en train de modifier la configuration des échanges internationaux dans ce domaine. Elles rendent possible la mise à disposition à bas prix de contenu, sous des formes audio et visuelles, ce qui a suscité un développement spectaculaire du mode 1, l'offre internationale de services d'enseignement électronique. Aux États-Unis, le marché de l'enseignement électronique dépasse déjà 8 milliards d'USD et augmente à un taux moyen de $98 \%$ depuis cinq ans. Bien que la quasi totalité des clients de ce service reste des résidents américains, le potentiel mondial de l'enseignement électronique est énorme ; en effet, le coût de fourniture par Internet est à peu près le même pour un résident américain situé à proximité que pour un résident malaisien de Kuala Lumpur, quand l'infrastructure de technologies de l'information est en place. L'usage de toutes sortes de formes d'enseignement interactif et à distance se répand; conjugué souvent à une offre internationale croissante de services d'éducation et de formation, il représente un potentiel considérable [Source: www.sitrends.org].

\section{Services d'enseignement supérieur}

93. Le déplacement des étudiants en quête de formation dans les différents cycles de l'enseignement supérieur s'effectue entre pays de tous niveaux de développement : entre pays développés, des pays en développement aux pays développés et vice versa mais, aussi entre pays en développement. Selon une enquête de la CEAP, la région asiatique constitue le principal point de départ des étudiants (46\%), tandis que l'Amérique du nord et l'Europe sont des destinations importantes.

94. Si l'essentiel des échanges de services d'enseignement supérieur a lieu entre les nations membres de l'OCDE (qui accueillent $85 \%$ des étudiants étrangers du monde), plusieurs pays en développement 
affirment fortement leur présence sur ce marché. Certaines institutions de ces pays, chinoises et sudafricaines par exemple, tout en cherchant surtout à inciter les étudiants à rester dans leur pays d'origine, tentent elles-mêmes d'étendre leurs activités à l'étranger. L'université indienne qui a pris le nom du Mahatma Gandhi possède une antenne dans les Émirats Arabes Unis [The Observatory on borderless education (Obervatoire de l'enseignement supérieur sans frontières) (2002)].

95. Au sein des pays en développement, la Malaisie est dans le peloton de tête des exportateurs de services d'enseignement. En 2000, quelque 26000 étudiants étrangers originaires de près de 100 pays, parmi lesquels l'Indonésie, la Chine, l'Inde et des pays de l'Asie de l'est et d'Afrique y étudiaient. Des accords d'équivalence existent avec des universités étrangères du Royaume-Uni, des États-Unis et d'Australie pour permettre aux étudiants d'obtenir des diplômes reconnus par ces pays dans un environnement où les coûts sont moins élevés [Source: MATRADE : Société de développement du commerce extérieur malaisien].

En Malaisie, à la fin des années 1980, le système d'enseignement supérieur a introduit une nouveauté : des accords de jumelage ont été passés avec des universités étrangères, permettant aux étudiants d'effectuer une partie de leur cursus dans leur pays et le reste à l'étranger. Pour répondre à la demande, tant nationale qu'étrangère, plus de 600 institutions d'enseignement privé sont apparues, allant des écoles secondaires aux universités ; des départements dépendant d'universités étrangères se sont également installés. Les accords de jumelage ont surtout été passés avec des universités britanniques, américaines, canadiennes, australiennes et néo-zélandaises. La qualité de l'enseignement fait l'objet d'une stricte surveillance. Les programmes des institutions supérieures privées sont soumis à des normes sévères fixées par l'Office national d'accréditation (NAB). Beaucoup d'organismes privés d'enseignement supérieur ont également été créé un service spécialement destiné à prendre soin des étudiants étrangers. Outre le haut niveau de l'éducation, la Malaisie présente un certain nombre de caractéristiques qui exercent un attrait sur les étudiants étrangers. On peut citer son climat ensoleillé, la diversité des cultures et des communautés, le coût de la vie raisonnable, les prix compétitifs des services d'enseignement, la sécurité, l'usage généralisé de l'anglais comme moyen de communication et une excellente qualité de vie. Les étudiants peuvent obtenir des diplômes dans des domaines aussi divers que les arts libéraux, le droit, l'informatique, la gestion des entreprises, les professions d'ingénieurs, les communications et les multimédias. Enfin, le système d'éducation de la Malaisie est très concurrentiel sur le plan des coûts, puisque les étudiants peuvent suivre les cursus jumelés avec des universités étrangères du Royaume-Uni, des États-Unis et d'Australie [Source : www.studymalaysia.com ]

96. De même, la Thaïlande, où l'enseignement est considéré comme un secteur vital d'exportation de services, a fait de gros efforts de publicité sur le plan international pour vanter la qualité des programmes offerts dans de nombreuses disciplines spécialisées: métiers d'ingénieurs, agriculture, santé publique, lettres, arts libéraux, entretien des forêts, sciences, gestion des entreprises et secteur touristique [Source: www.exporter.thaitrade.com].

Le collège Dusit Thani, tirant parti de la réputation internationale de la cuisine thaïlandaise, propose des licences d'administration des entreprises spécialisées en gestion hôtelière, tourisme et gestion en général. Outre ces programmes en quatre ans, le collège propose également de nombreuses formations ainsi que des préparations aux fonctions de direction réservées aux cadres moyens et supérieurs. Tous les cursus sont sous la responsabilité d'experts et de formateurs expérimentés, venant d'institutions d'enseignement renommées du monde entier, et bénéficient des meilleurs équipements. Dusit Thani dispose de salles de classes modernes dotées d'équipement audiovisuel, de laboratoires informatiques, d'une imitation de salle de réception, d'une vaste bibliothèque, d'un laboratoire de langues, d'une salle de démonstration et d'un grand auditorium. Les étudiants ont l'obligation de suivre 1000 heures de formation pratique qui s'ajoutent à leur programme théorique [Source: Internet].

97. En Amérique latine, il existe, dans un certain nombre de pays, une tradition ancienne de formation universitaire d'étudiants étrangers. L'Argentine profite de la forte baisse de son taux de change, à la suite de la crise financière, pour offrir l'accès à son système d'éducation de haut niveau international à des prix compétitifs. 
L'université de Belgrano, à Buenos Aires, est l'une des plus grandes d'Argentine ; elle reçoit des étudiants venant de tout le continent américain, mais aussi de I'Union européenne, d'Israël, de Nouvelle-Zélande, d'Australie, de Chine et du Japon. Elle propose des MBA aux normes internationales, des programmes d'études latino-américaines, l'apprentissage de l'espagnol pour les étrangers, des cours d'été sur l'environnement et l'anthropologie qui ont lieu en Patagonie (à La Angostura). L'université de Belgrano utilise largement l'argument de la récente crise financière argentine dans ses actions de marketing visant à attirer les étudiants étrangers. Elle ne fait pas seulement sa propre publicité en se présentant comme un établissement d'éducation abordable, mais de grande qualité ; elle offre aussi un programme diplômant sur la globalisation et les occasions qui en découlent pour les entreprises, considérées du point de vue argentin: l'accent est mis sur l'interdépendance accrue des États-Unis et d'autres pays ainsi que sur l'expérience argentine. On lit sur son site Internet : "Inscrivez-vous à un programme d'Executive MBA sur mesure, permettant une analyse sur le terrain, et vivez votre propre expérience d'une crise qui a marqué un tournant dans les méthodes internationales de redressement économique » [Source : étude de l'OCDE].

La petite Université pédagogique nationale de Bogota exporte des programmes d'enseignement supérieur et des services de conseil en matière éducative, en vertu d'accords passés avec des institutions similaires du Chili, d'Argentine, du Brésil, du Mexique, de Cuba, d'Espagne, d'Italie et d'Allemagne. A l'heure actuelle, son activité internationale est très modeste (pas plus de $10 \%$ du total), mais son représentant, Mme Eliska Krausova, prévoit que l'intensification des efforts d'internationalisation va faire augmenter ce pourcentage. Elle estime que les principaux obstacles sont l'obtention de visas et les restrictions légales concernant l'approbation des programmes [Source : étude de l'OCDE].

L'Institut technologique d'études supérieures de Monterrey (ITESM), généralement connu sous le nom de Tec de Monterrey, a été créé en 1943 par un groupe d'entrepreneurs ayant à sa tête Don Eugenio Garza Sada. Cette université mexicaine a acquis une position dominante en Amérique latine : elle compte 3500 étudiants étrangers, un corps enseignant international comprenant des professeurs associés de renom, neuf spécialisations, un programme d'enseignement virtuel très vanté et 15 bureaux de liaison aux États-Unis, au Canada, en Europe et en Amérique latine [Source: magazine Latin Trade et Internet].

98. En Afrique, l'Ouganda, l'Afrique du Sud et le Kenya sont dotés d'institutions d'enseignement post-secondaire qui ont formé de nombreux dirigeants régionaux du monde des affaires et de la politique.

\section{Services de formation}

99. A côté des services traditionnels d'enseignement universitaire diplômant, on a également assisté à une forte croissance internationale d'autres formes d'éducation et de formation; c'est ainsi que la formation aux technologies de l'information se développe particulièrement vite.

En 1981, un groupe d'informaticiens de Delhi, Rajendra S. Pawar, Vijay K. Thadani et P. Rajendran ont créé ensemble la société NIIT, spécialisée dans la formation aux TI, les logiciels et les solutions basées sur le savoir ; elle s'adresse à la fois aux particuliers et aux entreprises. Depuis l'origine, le projet de la société est « de rapprocher les gens et les ordinateurs dans un pays qui s'éveille tout juste aux $\mathrm{TI}$ » ; en outre, sa stratégie a été de privilégier l'enseignement de masse à l'informatique, y compris en initiant aux TI les enfants des milieux les plus défavorisés. Aujourd'hui, NIIT est devenue une entreprise internationale, présente dans 38 pays, et figurant parmi les 10 principaux exportateurs indiens de logiciels. Elle compte plus de 1000 entreprises clientes avec lesquelles travaillent ses filiales contrôlées à $100 \%$, disséminées dans 38 pays (zone Asie-Pacifique, Europe, Japon et États-Unis). NIIT privilégie la formation avancée sur diverses plates-formes de logiciels et la préparation de professionnels "immédiatement employables ", auxquels elle donne les compétences nécessaires pour faire carrière dans les TI. NIIT pratique l'enseignement à distance/électronique, qui est dispensé par NetVarsity, son université en ligne. La moitié de son chiffre d'affaires est réalisé aux États-Unis, en Europe et dans la région Asie-Pacifique. En 2002, le chiffre d'affaires mondial a été de 7.9 milliards d'INR [Source : Internet].

IPSEL Training est un département de la société Indo-Pacific Software and Entertainment, qui a noué une alliance et des accords techniques avec la société allemande Mindrome Technologies. II offre des technologies de certification de haut de gamme ainsi que des formations aux procédures douanières à des personnes, sociétés et administrations. IPSEL est une société cotée, qui a des bureaux et des franchisés dans toute l'Inde, de même que des associés pour la formation dans le monde entier. En 2002, son chiffre d'affaires a été de 61.52 millions d'INR in 2002 [Source Evalueserve]. 
Le Centre d'amélioration des performances de gestion, situé en Roumanie, est un organisme non étatique de conseil en gestion et en formation, tourné à la fois vers le marché interne et l'extérieur. Créé en 1992, il est le fruit d'une initiative commune d'une entité officielle britannique, le "Fonds pour le savoir-faire », des ministères roumains de l'industrie et du développement, de l'université du Hertfordshire et de l'université polytechnique de Bucarest. Le Centre a des clients au Royaume-Uni, au Danemark, en Belgique, en Irlande, en France, aux Pays-Bas et en Allemagne. II offre des programmes très intensifs et interactifs dans de nombreux domaines touchant à la gestion et à d'autres sujets : stratégie, efficacité, marketing et vente, ressources humaines, qualité totale, finance, dynamique de groupe, résolution des conflits et formations pour l'administration publique [Source : étude de l'OCDE].

Des spécialistes cubains de la gastronomie et de la gestion hôtelière dispenseront, au deuxième semestre de 2003, des cours à Luanda, capitale de l'Angola, et dans les provinces de Huila, Benguela et Cabinda. Le directeur de l'association des hôtels-restaurants et entreprises similaires de Luanda (Horsil), Joao Gonçalves, a révélé que ce projet résultait de contacts entre les gouvernements angolais et cubain. L'objectif est d'aider les institutions locales à réaliser leur projet de former un personnel plus nombreux, afin que les services hôteliers du pays soient bien préparés à répondre à la demande. Selon lui, la mesure vise aussi à former en Angola des professionnels de la gestion hôtelière en vue du festival international de gastronomie qui doit avoir lieu en 2005 dans ce pays [Source : Cubanet]. 


\section{SERVICES FINANCIERS}

\section{Vue d'ensemble}

100. Selon la classification de l'AGCS (W/120), les services financiers comprennent les assurances et les services relatifs à l'assurance, de même que les services bancaires et les autres services financiers (y compris l'information financière).

101. Les exportations de services financiers des pays en développement sont souvent le fait de coentreprises ou de filiales de grandes sociétés financières ou de compagnies d'assurance des pays de l'OCDE. Non seulement les filiales créées dans les pays en développement fournissent des services à la maison mère et au marché local, mais elles exportent, notamment vers les marchés d'autres pays en développement. Il convient de noter que, dans plusieurs exemples cités dans cette partie, l'entreprise participe également à diverses activités de la maison mère qui pourraient être classées dans la catégorie des autres services aux entreprises. Toutefois, elles ont été incluses ici en raison de leur composante de services financiers.

\section{Services bancaires et autres services financiers}

102. Plusieurs grandes sociétés de services financiers de pays de l'OCDE ont pris la décision stratégique d'investir dans des entreprises de pays en développement, à la fois pour améliorer l'efficacité de leurs opérations et se lancer sur de nouveaux marchés, comme ceux des autres pays en développement de la région. Ainsi, le secteur bancaire américain aurait économisé jusqu'à présent 6 à 8 milliards d'USD en sous-traitant certains travaux en Inde et aussi grâce à des progrès qualitatifs et de productivité. De fait, certains experts ont calculé qu'une banque occidentale standard pouvait sous-traiter 17 à $24 \%$ de ses sources de coûts, réduisant ainsi son coefficient d'exploitation de 6 à 9 points et doublant fréquemment ses bénéfices [Source : Evalueserve].

La société E-Serve International, dénommée auparavant Citicorp Securities and Investments, a commencé à opérer en Inde en 1992. Sa raison d'être est de fournir des services dépendant des technologies de l'information au secteur financier international et de répondre aux besoins des sociétés travaillant dans la banque, l'assurance, les marchés de capitaux, les fonds de placement et les marchés électroniques. Elle assure par exemple l'exécution des transactions, le traitement des chèques et les services de cartes de crédit et de débit. Elle emploie environ 3000 personnes et a réalisé en 2002 un chiffre d'affaires à l'exportation de 1947 millions d'INR au titre de services liés aux TI. E-Serve a une clientèle internationale, qui comprend notamment des entités dépendant de Citigroup situées dans plusieurs régions du monde, telles que l'Asie, l'Afrique du Sud et l'Europe [Source: Evalueserve].

Le concept d'un grand prestataire européen de services financiers est la collaboration avec des distributeurs et gestionnaires de fonds indépendants pour offrir des services novateurs de classe internationale. Alors que la plus grande partie de la chaîne de valeur est sous-traitée, la société concentre ses activités dans certains domaines définis comme le cœur de ses compétences (mise au point de produits, marketing, sélection des fonds et de leurs gestionnaires, aide aux distributeurs). La société est présente dans des pays en développement (Chili, Chine, Colombie, Chypre, Mexique et Émirats Arabes Unis) où elle possède de nombreuses filiales. Ces dernières ont pour vocation de faire la liaison avec les gestionnaires externes de fonds sur place, de sous-traiter les segments de la chaîne de valeur à forte intensité de main-d'œuvre d'autres filiales situées dans des pays où le coût du travail est élevé et de conseiller les entreprises. La filiale colombienne vend des services de conseil à ses homologues du Chili, du Mexique, de Suède et des États-Unis ; elle sous-traite également des fonctions liées aux TI pour le compte des filiales mexicaines et américaines [Source : étude de l'OCDE]. 


\begin{abstract}
Une des principales sociétés indiennes de TI et une grande banque européenne ont créé une co-entreprise, dont le siège social se trouve à Bangalore en Inde. Au fil des années, elle est devenue un puissant prestataire de services bancaires et financiers centrés sur les TI, qui emploie plus de 450 professionnels des logiciels et de la banque répartis entre Bangalore, Singapour, Francfort, New York et Londres. Elle a aussi acquis une vaste expérience et obtenu d'excellents résultats dans la fourniture de services de TI à la grande banque européenne dont elle fait partie. La société offre des solutions complètes en matière d'application et de développement. En outre, elle est en mesure d'apporter des connaissances professionnelles approfondies dans des domaines comme les financements commerciaux, les services de conservation de titres, la gestion de fortune, celle d'actifs et la banque électronique, qu'elle associe à des compétences dans les $\mathrm{TI}$ pour rendre des services de grande qualité avec un bon rapport coût/efficacité. L'infrastructure dont elle dispose comprend un moderne Centre d'excellence, à la pointe du progrès, qui occupe plus de 25000 mètres carrés de surface de bureaux à Bangalore. Cette co-entreprise donne à la société indienne de TI une base solide pour renforcer sa présence internationale dans les services financiers, qui constituent l'un des principaux marchés utilisateurs finaux des TI [Source : étude de l'OCDE].

La société Reuters Hong Kong propose des informations financières ainsi que des services de données et de nouvelles à des antennes étrangères de banques et à des industriels de Chine, de Taïwan et de Hong Kong, qui ont besoin de se renseigner sur l'ensemble de l'espace chinois. Elle vend également ses services à des sociétés internationales désireuses de faire des affaires avec des entreprises chinoises sans installer une représentation sur place. Enfin, elle renseigne les branches locales d'entreprises de toute l'Asie, des États-Unis, d'Europe et d'Australie [Source : étude de l'OCDE].
\end{abstract}

103. On dispose d'autres exemples d'exportations de services financiers de pays en développement qui montrent où ils sont parvenus à faire naître des activités financières sur place; c'est notamment le cas du vigoureux marché du micro-financement qui existe dans ces pays.

En 1984, le programme pour les entreprises rurales du Kenya a démarré sous la forme d'un projet à cinq ans visant à satisfaire les besoins financiers, de gestion et techniques d'ONG travaillant au développement de micro-entreprises et de petites entreprises. K-Rep est devenu un organisme respecté de micro-financement multi-fonctionnel, qui est prêt à relever les défis de ce secteur en Afrique au $21^{\text {ème }}$ siècle. L'ancien projet temporaire financé par USAID a donné naissance à un groupe de trois sociétés autonomes : l'agence de développement K-Rep, la banque du même nom et la société de conseil K-Rep, qui exporte ses services dans toute l'Afrique. Le directeur général du groupe, John Kashangaki, est optimiste sur l'avenir du secteur des micro-financements, grâce à l'apparition de banques de village qui jouent le rôle de prêteur principal. Ces banques et les associations de services financiers sont des institutions gérées localement dans lesquelles les petits entrepreneurs peuvent prendre des participations et espérer un certain rendement [Source : The East African Evening Standard, 31/03/2003]

104. Il arrive aussi que les prestataires de services financiers parviennent à occuper un créneau sur les marchés offshore. A l'Ile Maurice, des activités extra-territoriales se sont développées, parmi lesquelles des fonds de placement, des sociétés de portefeuille et des transactions internationales. En 1999, la CNUCED a estimé que les retombées directes ou indirectes de ce secteur représentaient $2.5 \%$ du PIB. Un accord de double imposition avec l'Inde, qui coïncidait avec la libéralisation de l'économie indienne et le lancement d'activités extra-territoriales à Maurice, a permis de canaliser des investissements vers l'Inde. Six milliards d'USD d'investissements auraient été effectués en Inde par l'Ile Maurice, ce qui représente $32 \%$ des entrées d'investissements étrangers directs et de portefeuille dans ce pays (chiffres de 1999). Plus récemment, le secteur extra-territorial mauricien s'est enrichi d'activités d'assurance captives ainsi que d'un centre d'enregistrement d'avions et d'une structure de financement pour l'hypothèque et la location des appareils et de pièces détachées. Les principaux facteurs de réussite ont été l'excellente image de marque donnée par la qualité des services touristiques, la situation géographique du pays, son appartenance aux blocs régionaux et l'existence d'un cadre juridique réglementaire et fiscal sain [Source : CNUCED et MOBAA]

105. La mise en place de services bancaires par Internet, le commerce électronique et la création de systèmes de paiements sécurisés et d'autres fonctions d'appui au commerce électronique pourraient aussi constituer des créneaux. Mais les services d'exécution des paiements électroniques dépendent d'un dosage réussi de technologies avancées et de compréhension du marché ainsi que des cadres juridiques qui les réglementent et définissent leurs possibilités. Pour exporter ces services, on doit souvent donner des 
preuves crédibles de sa capacité à répondre aux besoins du marché ; cela signifie que la réussite à l'exportation peut être conditionnée par les résultats obtenus sur des marchés développés. Ce facteur risque de freiner un certain nombre de pays, au même titre qu'une mauvaise perception de la capacité des fournisseurs de pays en développement à offrir des solutions de haute technologie.

DBS Bank est la banque de prestige de DBS Group Holdings à Singapour. Elle fait partie des premières banques d'Asie et occupe le $70^{\text {ème }}$ rang à l'échelle mondiale. Renommée dans le secteur des services bancaires par Internet et du commerce électronique, elle occupe la première place sur le marché des prêts et dépôts en dollars de Singapour et de la mobilisation de capitaux. En dehors de Singapour, le groupe DBS offre ses services à des entreprises, des institutions et des particuliers par l'intermédiaire de ses filiales de Hong Kong Chine, des Philippines, d'Indonésie et de Thaïlande et propose des services bancaires internationaux grâce à un réseau de 13 succursales et bureaux à l'étranger [Source : www.dbs.com.sg].

Decidir, société mexicaine offrant des prestations d'entreprise à entreprise sur Internet, assure des services de paiements sécurisé et d'authentification à environ 130000 entreprises d'Argentine, du Brésil, du Chili et du Mexique. Ses principaux clients comprennent Banco Rio, Citibank, Peugeot, Unilever et American Express [Source http//:corp.decidir.com].

Une société africaine, qui exporte maintenant des services de paiements sécurisés, en Afrique comme sur les marchés d'Europe et d'Amérique du nord, rapporte que la principale difficulté initiale a été de vaincre dans son propre pays le scepticisme à l'égard de la capacité de compatriotes à mettre au point et à utiliser des technologies de pointe. De tels comportements, assez caractéristiques des économies en développement, pénalisent les entrepreneurs locaux aptes à recourir à la technologie pour répondre aux besoins du marché, et jouent en faveur de leurs concurrents des pays développés [Source : étude de l'OCDE].

\section{Services d'assurance}

106. A l'instar des autres prestataires de services bancaires et financiers, plusieurs grandes compagnies d'assurance ont créé des filiales dans des pays en développement ; ces dernières exportent des services à la fois vers les bureaux de la société mère dans le monde entier et vers d'autres clients de divers pays.

GE Capital International Services (GECIS) a été fondée en 1996 avec le statut de filiale à $100 \%$ de la société GE Capital Services India. Installée à Gurgaon, Hyderabad, Bangalore et Jaïpur, elle emploie quelque 13000 personnes. Le chiffre d'affaires prévu au titre du traitement des procédures d'assurance était de l'ordre de 300 millions d'INR. La société s'occupe de l'exécution d'opérations d'assurance et de banque pour les entreprises du groupe et pour d'autres clients comme J.C. Penny. Ses responsabilités comprennent aussi bien des travaux à faible qualification d'entrée de données et de service clientèle que des fonctions de direction exigeant de grandes compétences. Les services à haute valeur ajoutée sont la souscription d'assurances et l'actuariat. Au centre d'Hyderabad, GE a employé 10 ingénieurs en génie civil spécialisés dans la souscription d'assurances contre les inondations. Les services fournis par GECIS peuvent être généralement classés dans les catégories de l'exécution de transactions, de la comptabilité internationale et des centres d'appels [Source: Evalueserve].

EXL Services (I) Pty. Ltd. qui avait commencé comme jeune pousse du secteur indien des assurances offrant des services pour les tiers, a rapidement été rachetée par la grande compagnie d'assurance américaine Conseco. Vers la fin de 2002, elle a repris ses activités d'assureur de tiers et est actuellement l'un des principaux vendeurs de procédures complètes d'assurance en Inde. Ses activités sont réparties entre trois sites dans la ville de Noida et elle compte actuellement environ 1900 salariés. Les services financiers qu'elle offre sont les contrats d'agents, le règlement des sinistres, la comptabilité, la gestion des dettes, la souscription à de nouvelles assurances, le versement et l'administration de rentes viagères. La société est titulaire aux États-Unis d'une licence de tiers administrateur, qui l'autorise aussi à faire souscrire. Elle détient des licences pour la souscription de polices d'assurance dans environ 30 états des États-Unis [Source : Evalueserve]. 
Le centre de services partagés de Suisse Ré a été créé dans la ville indienne de Bangalore, en août 2000, pour faire bénéficier de cette méthode les activités administratives de réassurance. Cette initiative s'inscrivait dans le cadre d'une concentration des procédures d'exécution, visant à exploiter des synergies et à améliorer les résultats des départements d'assurance non-vie. L'investissement initial était de l'ordre de CHF 4 millions. Le centre de Bangalore s'occupe actuellement de procédures comme la gestion des contrats, celle des sinistres et la comptabilité de la réassurance pour les marchés de Chine, d'Australie/Nouvelle-Zélande, d'Asie du Sud-Est, du Japon, de Corée, d'Inde et des pays arabes; il travaille également pour le siège de Zurich. Son chiffre d'affaires actuel est estimé à CHF 2 milliards et il emploie 70 à 80 personnes [Source : Evalueserve].

Le groupe American International (AIG) et la compagnie PhilAm Life Philippines ont créé une co-entreprise (à $99.99 \%$ américaine) pour mettre en place une unité utilisant les technologies de l'information. Cette dernière emploie actuellement 484 salariés. Elle est surtout chargée des procédures d'assurance-vie d'AIG, c'est-à-dire du centre d'appels, de l'enregistrement des données et du codage, de la transcription des formulaires de demande et de la reproduction sous forme d'images, du traitement des sinistres, de la préparation des chèques, de la comptabilité et des paiements. La société, enregistrée auprès de l'Autorité de la zone économique des Philippines et installée dans un parc de TI, a commencé ses activités en décembre 2002. AIG a consenti à un investissement initial de 3.6 millions d'USD [Source : Evalueserve].

107. En Amérique latine, les réassureurs collaborent avec les prestataires de services financiers et les assureurs pour proposer une série de nouveaux produits concurrentiels. On peut citer l'alliance stratégique de Swiss Reinsurance avec Enhance et Banco Pactual S.A. (et avec la filiale brésilienne de cette dernière Seguradora Brasileira de Fiancas S.A.). Cette alliance offre des assurances cautionnement et des montages sophistiqués à base de crédits pour assurer des opérations de privatisation et de grands projets d'infrastructures au Brésil ainsi que dans d'autres pays d'Amérique latine. En Colombie, la Compañía Central de Seguros S.A., située à Bogota, est une compagnie d'assurance employant plus de 260 personnes, qui travaille surtout avec des réassureurs étrangers (Mexique, Panama, États-Unis, Espagne, Suisse et Allemagne). Son activité internationale est très importante (jusqu'à $75 \%$ du total) [Source : étude de l'OCDE].

108. L'assurance santé est aussi un domaine où l'Amérique du Sud exporte. Les compagnies d'assurance brésiliennes ont des clients en Colombie et les chiliennes au Mexique [Source : OMS]. 


\section{SERVICES DE SANTÉ}

\section{Vue d'ensemble}

109. Le secteur de la santé comprend les services médicaux, dentaires, infirmiers, paramédicaux, hospitaliers, sociaux et les autres services de santé humaine. C'est l'un des domaines de l'économie mondiale où la croissance est la plus rapide. On estime qu'il génère un chiffre d'affaires de 3000 milliards d'USD par an dans les pays de l'OCDE, qui pourrait atteindre 4000 milliards d'USD en 2005. La mondialisation des services de santé se manifeste de plusieurs manières : augmentation de l'offre en provenance de l'étranger (par courrier traditionnel, courrier électronique et autres moyens), mouvement des prestataires et des consommateurs ; développement des co-entreprises et des accords de coopération.

\section{Mode 1}

110. La fourniture transfrontières (mode 1) comprend l'envoi d'échantillons de laboratoire, de diagnostic et d'une seconde opinion médicale ainsi que la consultation par les canaux habituels de la poste ou par des moyens électroniques. Des pays situés dans diverses zones géographiques offrent un nombre croissant de services de santé sur Internet, tandis que la télémédecine se développe en Asie et en Amérique latine. Ainsi, la Chine propose des diagnostics en ligne à des patients de Taiwan, de Macao et de certains pays d'Asie du Sud-Est, [Source: CNUCED/OMS]. En Inde, cinq radiologues travaillant pour la société Wipro. interprètent quotidiennement 30 examens par scanner pour le compte de l'hôpital général du Massachusetts [Source: BusinessWeek]. De plus en plus d'échantillons médicaux provenant d'Amérique centrale sont adressés au Mexique pour y être diagnostiqués. Beaucoup d'organismes médicaux font transcrire numériquement leurs dossiers ou les entretiens avec les patients au Bangladesh, en Inde, au Pakistan, aux Philippines ou au Zimbabwe.

Max Health Scribe est une co-entreprise fondée en 1992 par la société Max India et son homologue américaine HealthScribe. Elle a commencé ses activités à Bangalore, en 1994, et a été la première société indienne de transcription médicale. Elle emploie actuellement plus de 1100 spécialistes du vocabulaire médical et 40 médecins lui sont attachés. Elle travaille avec des hôpitaux et des cliniques de première importance aux États-Unis ; son chiffre d'affaires a atteint 7.8 milliards d'USD au cours de l'année financière 2001-2002 [Source : Evalueserve].

111. Il est probable que l'apparition d'un secteur du «bien-être», prévue par Paul Zane Pilzer (l'économiste qui avait prédit le succès d'Internet), qui y voit la grande industrie de l'avenir, va offrir de nouveaux créneaux aux services d'aide. Les professionnels de la santé en auront besoin pour recentrer leur expertise du traitement des maladies vers la prévention. Aux États-Unis, par exemple, les praticiens proposent déjà à leurs patients des sites Internet spécialisés qui leur donnent accès à des outils d'auto-examen, à des aides au traitement de maladies chroniques, à leurs propres dossiers médicaux sur Internet, à des informations médicales sur mesure et à beaucoup plus encore. L'existence de plus de 20000 sites Internet d'information médicale offre des possibilités aux fournisseurs de contenu, tandis que le tournant vers la médecine électronique donne toutes sortes de moyens de consultation [Source : Centre du commerce international]. 


\section{Mode 2}

112. La consommation à l'étranger (mode 2) se réfère au mouvement des consommateurs, en quête de diagnostic et de traitement, vers le pays qui fournit le service. En usant de ce mode, les patients recherchent dans des pays avancés ou en développement des traitements de grande qualité à des prix abordables ou des formes nouvelles de médecine et de soins. Plusieurs pays en développement exportent des services de santé sous forme de consommation à l'étranger. Ainsi, dans les pays du MERCOSUR (Argentine, Brésil, Paraguay, Uruguay), la création de la "carte MERCOSUR » permet aux adhérents de coopératives médicales d'un pays de se faire soigner dans un organisme affilié d'un autre pays du MERCOSUR.

113. Cuba s'est attaché délibérément à attirer dans ses hôpitaux hautement spécialisés des patients étrangers en provenance d'Amérique latine, des Caraïbes, d'Europe et de Russie. Le pays a également diversifié son offre en privilégiant la mise au point de nouvelles procédures et de nouveaux médicaments ainsi que le traitement de certaines maladies de peau incurables dans d'autres pays, comme la rétinopathie pigmentaire ou le vitiligo, affection qui détruit le pigment de la peau et laisse des taches blanches. On estime qu'en 1995-1996 plus de 25000 patients étrangers sont allés se faire traiter à Cuba, ce qui a donné lieu à des ventes de services de santé d'un montant d'environ 25 millions d'USD [CNUCED/OMS]. Les cliniques du Costa Rica font état d'une augmentation de la clientèle originaire du Royaume-Uni, de Norvège, du Canada et des États-Unis [Source: Guardian 16/12/2000]. Le Chili accueille aussi des patients à revenus moyens et élevés qui viennent de Bolivie et du Pérou et, dans une moindre mesure, d'Equateur [Source : Organisation panaméricaine de la santé/OMS].

Cira Garcia, une clinique attenante au quartier aisé de Miramar, à La Havane, a accueilli en 2000 près de 1300 étrangers en tant que malades hospitalisés, et des milliers d'autres en tant que malades externes. A des prix qui sont inférieurs d'environ un tiers à ceux pratiqués aux États-Unis, elle offre toutes les prestations possibles, du traitement d'une hernie discale (pour 4570 dollars, anesthésie et séjour de deux semaines à l'hôpital compris) à de la chirurgie oculaire au laser, en passant par des opérations de liposuccion. Les patients qui règlent leur note en dollars bénéficient de "toutes les commodités des cliniques les plus modernes ", soulignent les dépliants, notamment de la télévision par câble, de la climatisation et d'un service de télécopie international fonctionnant 24 heures du 24 . La plus prisée des prestations proposées par Cira Garcia est la chirurgie plastique. Environ 80 pour cent des patients de cet établissement viennent d'Amérique latine et des Caraïbes, mais il attire des clients de pays aussi lointains que le Japon et la Finlande. [Source : Cubanet, 2001].

L'Inde est également un gros exportateur de services de santé par le biais de la consommation à l'étranger. Des patients venus du Bangladesh, de l'est du bassin méditerranéen, des États-Unis, du Népal, du Royaume-Uni et du Sri Lanka se rendent en Inde pour y subir des opérations chirurgicales et bénéficier de services spécialisés, notamment en matière de neurologie, de cardiologie, d'endocrinologie, de néphrologie et d'urologie. Ils y sont attirés par des soins de haute qualité dispensés à des prix abordables. Le coût des services médicaux en Inde est compris entre un dixième et un trentième de leur coût aux États-Unis. Ainsi, une opération de pontage, qui est facturée 13000 dollars aux États-Unis, ne coûte que 800 dollars en Inde, sur la base des mêmes technologies et équipements. Le Ministre britannique de la santé a même évoqué la possibilité d'emmener par avion des patients britanniques en Inde pour qu'ils y soient soignés, afin de réduire le fardeau que représente pour l'État la prise en charge des soins médicaux. [Source : Financial Times]

Le groupe Apollo Hospitals est la plus grande chaîne hospitalière privée indienne ; elle est présente dans l'assurance-maladie, les pharmacies et la formation. En activité depuis près de 20 ans, elle dispose d'installations médicales à Chennai, Hyderabad, New Delhi et Dubaï. L'hôpital offre plusieurs formules de distribution de services, dont une réservée spécialement aux non-résidents qui a pour vocation d'offrir des soins de qualité à prix réduit [Source : Evalueserve]. 
L'Afrique du Sud est devenue une des principales destinations des patients en quête de chirurgie plastique. Le site web de l'Association of Plastic and Reconstructive Surgeons of South Africa (Association des spécialistes en chirurgie plastique et reconstructive d'Afrique du Sud) contient des informations spécifiquement destinées aux visiteurs étrangers qui se rendent dans ce pays pour y subir des opérations de chirurgie esthétique. Ces renseignements portent notamment sur la manière de trouver un chirurgien et de préparer une opération, ainsi que sur les hôpitaux, l'anesthésie, et les frais à engager. Une rapide recherche sur Internet a fait apparaître plus de 20 cliniques proposant des services de chirurgie cosmétique aux étrangers en visite en Afrique du Sud. Parmi les raisons avancées par les patients pour faire un tel choix figurent la haute qualité des prestations conjuguée à leur faible coût, et l'avantage de pouvoir subir l'opération désirée en toute intimité pendant leurs vacances. On ne dispose pas de chiffres exacts, mais des centaines de Britanniques au moins ont fait l'objet d'interventions de chirurgie cosmétique en Afrique du Sud ces dernières années. [Source : The Guardian, 16/12/2002].

Surgeon et Safari, une des entreprises d'Afrique du Sud qui ont le mieux réussi à associer chirurgie et tourisme, déclare qu'elle reçoit 20 à 30 personnes par mois et que le nombre des clients augmente très rapidement. $90 \%$ d'entre eux - moitié de britanniques, moitié d'américains - subissent des opérations esthétiques. Afrisurge, autre entreprise sud-africaine, propose des services analogues, mais, de plus en plus prend aussi en charge des clients qui se rendent dans des cliniques spécialisées dans le traitement des migraines, les problèmes dentaires et les troubles oculaires. De fait, l'Association sud-africaine des dentistes et un organisme touristique du Cap signalent la montée du «tourisme dentaire» [Source : www.safrica.info]. D'autres sociétés (Mediscapes par exemple) proposent de nombreux services médicaux, notamment en matière de cardiologie, de traitement de la toxicomanie, de soins gastrointestinaux et de traitement de la stérilité [Source : "Sun surf and surgery ", 24/7/2002, disponible sur le site www.safrica.info].

Un des moyens de promouvoir l'Afrique du Sud comme destination pour la chirurgie esthétique a été l'association d'un des plus grands hôpitaux du pays et d'un site d'enchères sur Internet; l'objectif est de vendre sous cette forme des opérations de chirurgie esthétique, une opération différente étant mise aux enchères chaque jour de la semaine [Source: BBC en ligne, 24/7/2000].

114. Plusieurs pays en développement se sont également diversifiés dans des domaines tels que l'enseignement médical et paramédical, le tourisme de santé et la médecine non conventionnelle. Ainsi, en Thaïlande, l'hôpital universitaire Pramongkutklao et d'autres écoles de médecine ont des places réservées pour des étudiants d'autres pays en développement, tandis que certaines institutions médicales thaillandaises proposent des formules qui conjuguent soins et tourisme. La Thaïlande exporte des médecines traditionnelles au sein de la région et dans le monde entier. L'Indonésie importe des médecines traditionnelles orientales de Chine, de Taïwan et de Corée et exporte la médecine traditionnelle javanaise, appelée jamu, chez ses voisins. En Inde, l'Ecole Ayurvedic de Kottakkal, au Kérala, est un centre de soins holistiques qui reçoit des patients venant de la zone orientale de la méditerranée, d'Allemagne, de Malaisie, du Royaume-Uni et des États-Unis. En Malaisie, les voyagistes élaborent des formules de services médicaux au choix du patient et d'hospitalisation dans les 49 centres médicaux privés réservés aux étrangers [Source : MATRADE - Société de développement du commerce extérieur malaisien].

\section{Mode 3}

115. La présence commerciale à l'étranger (mode 3) implique l'établissement d'hôpitaux, de cliniques, de centres de diagnostic et de traitement ainsi que d'infirmeries. L'Inde, l'Indonésie, le Népal et le Sri Lanka font partie des pays qui s'ouvrent de plus en plus aux investissements étrangers. Gleaneagles International est une co-entreprise qui a été montée par des associés situés en Inde, en Indonésie, en Malaisie, au Sri-Lanka et au Royaume-Uni, dans le but de lancer une chaîne internationale d'hôpitaux. Un autre important groupe hospitalier indien a prévu de construire, d'ici à 2008, 1000 nouveaux hôpitaux en Inde, au Sri Lanka et au Népal : 16 d'entre eux, situés dans les grandes métropoles, prodigueront des soins extrêmement spécialisés ; 32 hôpitaux spécialisés prendront place dans d'autres grandes villes ; le reste se trouvera dans de petites agglomérations.

116. La Chine pratique activement l'installation à l'étranger de cliniques à capitaux totalement chinois ou ayant un statut de petites co-entreprises. A la fin de 1995, il existait une centaine d'établissements hospitaliers régis par l'une ou l'autre formule, situés dans un peu plus de 20 pays hôtes d'Asie, du MoyenOrient et d'Europe (y compris l'ancienne URSS et les pays de l'est) ainsi qu'aux États-Unis. Ils sont de 
taille relativement réduite, mobilisent environ 200000 à 300000 USD et pratiquent généralement la médecine chinoise traditionnelle (par exemple l'acupuncture et la moxibustion) [Source : CNUCED]. Au Brésil, la Chine a investi dans une cinquantaine d'entreprises pour un montant de capitaux de l'ordre de 50.3 millions d'USD. Ces investissements ont surtout été affectés à d'autres activités (agroalimentaire, fabrication de vêtements et de certains types de produits industriels légers, fonderie, développement forestier, traitement du bois, transport), mais ils ont aussi porté sur les services médicaux et sanitaires [Source : site Internet du gouvernement chinois].

\section{Mode 4}

117. Les échanges de services de santé s'effectuent aussi par le biais de déplacements du personnel médical (mode 4): médecins, spécialistes, infirmières, paramédicaux, sages-femmes, techniciens, consultants, formateurs, gestionnaires de santé et autres professionnels. On inclut dans les mouvements des professionnels de la santé les flux temporaires et permanents.

118. Si la migration permanente est essentiellement un phénomène nord-sud, suscité par les écarts de salaires et l'attente d'une amélioration des niveaux de vie, la mobilité temporaire résulte surtout d'accords bilatéraux entre des gouvernements désireux de coopérer. Ainsi, la Chine et Cuba envoient en Afrique du personnel médical dans le cadre de contrats temporaires rémunérés. Un exemple très récent est le programme d'une durée de deux ans, lancé en février 2003, en vertu duquel 74 médecins et spécialistes de la santé ont été déployés dans les hôpitaux provinciaux de tout le Zimbabwe. Ce groupe de praticiens, qui comprend 50 généralistes et des spécialistes en urologie, dentisterie, gynécologie et pédiatrie devrait atténuer la pénurie criante de médecins que connaît le pays [Source: All Africa]. De même, le Ghana envoie des professionnels de la santé en Jamaïque et l'Inde dans la partie orientale du pourtour méditerranéen. Cette dernière contrée est un important marché d'accueil pour les médecins, infirmières, spécialistes des rayons $\mathrm{X}$, techniciens de laboratoire, hygiénistes dentaires, physiothérapeutes et rééducateurs en provenance de nombreux pays en développement. 


\section{SERVICES INTERNET}

119. La fourniture de services par Internet comprend à la fois l'accès à Internet (qui fait intervenir des services de télécommunications) et la fourniture de contenu, qui se compose souvent d'un assortiment de services aux entreprises, d'audiovisuel et de services informatiques et connexes. Si l'AGCS classe séparément l'instrument, c'est-à-dire le moyen d'acheminement, et le contenu, de nombreux fournisseurs d'accès à Internet offrent aussi du contenu. Comme il est difficile de distinguer ces activités et de décider dans quelle catégorie classer les entreprises, on a regroupé les services liés à Internet dans une partie séparée.

120. Les sociétés latino-américaines spécialistes d'Internet ont profité de la communauté de langues pour prendre pied dans les autres pays du sous-continent. Certaines d'entre elles, très désireuses d'étendre leurs activités en dehors de la région, ont de plus en plus ciblé les hispanophones des États-Unis, d'Espagne et du Portugal.

Starmedia est l'un des plus grands et des plus prospères prestataires de services Internet d'Amérique latine ; il est représenté dans 7 pays et a des millions d'abonnés dans l'ensemble de la région. De même, El Sitio, installé à Buenos Aires, travaille pour des millions d'internautes hispanophones et lusophones d'Amérique latine. Mercado Libre est un site d'enchères sur Internet [Source: New York Times, août 2000].

Universo Online International (UOL), dont le siège social est à Sao Paulo, est le plus grand fournisseur d'accès à Internet d'Amérique du Sud; il a des portails en Argentine, au Chili, au Venezuela, en Colombie, au Mexique et en Espagne. UOL, qui existe depuis 1996, fait étalage d'une masse de manœuvre financière croissante. II a comme actionnaires majoritaires Folha, le plus puissant groupe de presse du Brésil, et Abril, le principal éditeur de magazines. Son point fort au Brésil et à l'étranger a été d'accueillir des pages Internet en passant des accords d'exclusivité de contenu avec des groupes de médias locaux. A l'instar d'autres sociétés latino-américaines du secteur de l'Internet, UOL a été contraint d'étendre son activité à l'étranger, afin d'être rentable et de compenser la faiblesse du pouvoir d'achat et du taux de connexion au Brésil [Source : New York Times, août 2000 et Newsweek 1999].

La société PACnet, domiciliée à Singapour, tire près de la moitié de son chiffre d'affaires d'autres pays anglophones, situés surtout dans la zone Asie-pacifique (Hong Kong, Philippines, Thaïlande, Inde et Australie) et, dans une moindre mesure de pays asiatiques non-anglophones. Elle a pu opérer dans ces derniers grâce aux services de traduction que lui offre son associé israélien Babilon.com. Suite à l'acquisition en 2000 d'un voyagiste d'entreprise, PACnet a commencé à collecter des portails verticaux d'entreprise à entreprise qui permettent d'amener des sociétés traditionnelles sur Internet; sa division de commerce électronique, PACfusion sert de portail pour l'Asie. PACnet a ensuite élargi son offre de contenu à des services d'accès global en matière de correspondance électronique, aux produits et services en ligne de la banque Standard chartered ainsi qu'aux services de réservation en ligne d'Asiahotel.com. Larry Ang, président de PACfusion, explique que la stratégie de la société est de recruter des professionnels dans tous les pays où elle pénètre, afin de donner au contenu un caractère local [Source : Financial] Times]. 
TerraGroup est une société de portefeuille qui a été fondée en 1997 pour fournir au marché local, au Moyen-Orient et aux marchés internationaux un large éventail de produits et de services de télécommunications et d'Internet. Outre son siège social de Beyrouth, TerraGroup possède des bureaux dans diverses parties du monde. II tire sa force et sa position unique sur le marché de l'avantage que lui confère la conjonction des capacités spécifiques des entreprises qu'il contrôle. II se compose en effet de trois sociétés principales : TerraNet, TerraGate et TerraVision. TerraNet est un fournisseur d'accès à Internet, qui offre les principaux services disponibles sur le réseau. TerraGate est spécialisée dans la fourniture, l'intégration et l'installation de services de commerce et de banque électronique. TerraVision est une société de conception de sites et de solutions électroniques, dont la mission est d'utiliser pleinement le potentiel de la technologie Internet pour aider les entreprises à faire passer leurs activités sur le réseau, à obtenir un rendement positif de leurs investissements et à atteindre des positions dominantes sur le marché international [Source : http://corp.terra.net.lb].

Alibaba.com a son siège social à Hong Kong et sa base opérationnelle à Hangzhou (Chine) ; elle possède aussi une filiale au Japon, des bureaux en Californie, à Londres, à Shanghaï et à Pékin ainsi que 10 points de vente disséminés en Chine continentale. Elle se présente elle-même comme le plus grand marché pour les échanges internationaux et comme le principal fournisseur de services de marketing sur Internet aux importateurs et aux exportateurs. Le nombre de membres inscrits dépasse 1600 000, en provenance de plus de 216 pays, et il augmente de plus de 3000 par jour. Alibaba.com a été désigné par le magazine Forbes "numéro 1 du réseau B2B » et choisi comme site Internet d'entreprise à entreprise le plus populaire par les lecteurs de la « Far Eastern Economic Review » [Source: Internet].

www.amarillas.com, lancé en mai 2002 par la société Mercantil, est un portail latino-américain destiné aux PME ; il leur permet d'acheter et de vendre leurs biens et services dans toute la région ainsi qu'en Amérique du nord et en Europe. Ce portail, de même que www.mercantil.com, donne aux PME la possibilité de concevoir leur propre page d'accueil, de faire figurer la liste de leurs produits et services sur Internet et de commercialiser leurs marques dans le monde. Ces deux portails proposent une gamme d'autres services, notamment une rubrique d'actualités, des instruments pour trouver des clients et des études sur les marchés boursiers [Source: www.mercantil.com]. 


\section{SERVICES PORTUAIRES ET CONNEXES, SERVICES DE TRANSPORT MARITIME}

\section{Vue d'ensemble}

121. La plus grande partie du transport de marchandises s'effectue par mer. Un porte-conteneurs moderne fonctionnant à $80 \%$ de sa capacité transporte 3000 conteneurs nécessitant plus de 100000 documents [Source : Latin Trade Magazine].

\section{Services portuaires et connexes}

122. On trouve plusieurs pays en développement dans la liste des 20 plus grand terminaux de conteneurs du monde, qui représentent ensemble $54.34 \%$ de ce mode de transport maritime. On remarque en particulier que quatre des cinq terminaux ayant le plus fort débit se situent dans ces pays : Hong Kong est en tête, suivi de Singapour, du port de Kaohsiung au Taipeh chinois et de celui de Busan en Corée du Sud. Les autres pays en développement classés dans les 20 premiers terminaux sont la Chine (Shanghai), les Émirats Arabes Unis (Dubaï), la Malaisie (Port Klang), l'Indonésie (Tanjung Priok) et les Philippines (Manille) (voir Tableau A.3 de l'Annexe, Partie I). Si Shanghaï, Port Klang et Tanjung Priok, qui sont tous des ports de rang secondaire, ont connu une très forte progression du trafic de conteneurs, c'est parce que les alliances internationales de transporteurs ont commencé à proposer des formules de services multiples, se chevauchant, qui prévoient le débarquement uniquement dans les principaux centres portuaires ou dans les centres portuaires secondaires. En outre, les trois ports en question ont beaucoup investi en collaboration, avec le secteur privé, dans les infrastructures, les technologies améliorant l'efficacité et les systèmes de gestion [Source : CESAP].

123. Le Sri Lanka a également exploité avec succès ses avantages naturels en développant ses services portuaires et il est devenu une plaque tournante du trafic de conteneurs en Asie du Sud. Le secteur des transports joue un rôle croissant et dégage un excédent net en hausse au rythme de $13 \%$ depuis quelques années. La spécialisation délibérée en terminal de conteneurs s'est appuyée sur la mise en place de capacités internes, qui a permis des gains de productivité et une progression des recettes d'exportations. Pendant la période 1979-1997, le trafic a presque centuplé, à tel point qu'en 1993 le port de Colombo a été confronté à une demande supérieure à ses capacités de manipulation des conteneurs. Depuis lors, un nouvel effort d'expansion et de modernisation des équipements est en cours; on a également mis l'accent sur l'adaptation de la main-d'œuvre à l'utilisation des technologies modernes et de l'informatique [Source : CNUCED].

124. Le port de Dar-es-Salaam traite une grande partie du fret transporté par route et par rail à destination, et en provenance, de la Zambie, du Burundi, du Rwanda, de l'Ouganda et de la République démocratique du Congo. Les autorités portuaires de Tanzanie ont loué le terminal de conteneurs à un opérateur privé dans le cadre du programme de privatisation et, en 2002, le port de Dar-es-Salaam s'est doté de terminaux s'approchant des normes internationales et affichant un taux de productivité supérieur de $50 \%$ aux autres ports africains (comme celui de Mombassa au Kenya) ${ }^{12}$ [PricewaterhouseCoopers

12 La tendance à la libéralisation du secteur tanzanien des transports maritimes s'est récemment inversée. Après avoir essayé de faire de nouveau de l'activité d'agent maritime un monopole d'État, le gouvernement 
(2003a)]. La création à l'Ile Maurice d'une zone franche portuaire, destinée à être la plaque tournante du commerce maritime au sud et à l'est de l'Afrique, illustre la réussite d'une stratégie gouvernementale plus vaste visant à accroître et à diversifier les exportations de services [Source : CNUCED]. Au Maroc, la quasi totalité des échanges extérieurs emprunte la voie maritime. Les ports marocains enregistrent chaque année près de 14000 escales de navires qui chargent ou déchargent des cargaisons en provenance ou à destination de 120 pays. [Source : www.arabicnews.com].

125. Avant 1990, les ports argentins se distinguaient par des dysfonctionnements institutionnels (y compris un sérieux problème de corruption), un système inefficace de subventions croisées et l'insuffisance des investissements de modernisation. Les tarifs étaient parmi les plus élevés du monde. Dans les années 1990, l'État a mis en oeuvre une réforme globale qui a permis de quadrupler en dix ans le volume du trafic maritime (passé de 249000 à 1070 millions d'EVP). Le projet de réforme prévoyait deux dispositions importantes : la participation du secteur privé et l'encouragement à la concurrence sur les services portuaires. Dans le cas du port de Buenos Aires, six terminaux ont été concédés au secteur privé selon une procédure compétitive ; l'utilisation des infrastructures donne lieu au paiement d'un loyer à l'État, conformément au modèle de propriété portuaire. On a intensifié la concurrence en permettant la libre entrée sur le marché, tout opérateur étant autorisé à construire, entretenir et gérer un port à usage public ou privé. A la suite de ces réformes, les ports argentins sont devenus les moins chers d'Amérique latine et les investissements privés ont commencé à affluer. La productivité s'est vivement accrue, entraînant une diminution significative des coûts d'exploitation et du temps de stationnement dans les ports. La conjonction de ces facteurs et de ceux qui ont déjà été mentionnés s'est traduite par une réduction des prix globaux de manipulation des conteneurs dans les terminaux (voir tableau 2).

Tableau 2. Tarifs de manutention des conteneurs dans les terminaux argentins

\begin{tabular}{lll}
\hline \multicolumn{1}{c}{$\quad$ Indicateur } & $\mathbf{1 9 9 1}$ & $\mathbf{1 9 9 7}$ \\
Cargaison (en milliers de tonnes) & 4000 & 8500 \\
Conteneurs (en milliers d'EVP) & 300 & 1023 \\
Capacité (en milliers d'EVP) & 400 & 1300 \\
Grues (nombre) & 3 & 13 \\
Productivité (par salarié) & 800 & 3100 \\
Temps moyen d'un conteneur au port (en jours) & 2.5 & 1.3 \\
Coût par conteneur (USD/EVP) & 450 & 120 \\
Note : EVP, unité de mesure équivalant à 20 pieds & & \\
\hline
\end{tabular}

Source : B. Hoekman et al. (2000) « Development, Trade and the WTO : A Handbook », Banque mondiale.

126. Plusieurs sociétés de pays en développement se trouvent désormais au premier rang mondial pour la fourniture de services portuaires et connexes. Dans bien des cas, comme ceux des entreprises de Hong Kong Chine et de Singapour, elles sont parvenues à cette position grâce à la réussite des grands ports nationaux.

a adopté une nouvelle loi qui prévoit que toute entreprise demandant une licence d'agent maritime doit appartenir à un ressortissant tanzanien ou, conformément à l'arrêté sur les sociétés, être détenue à plus de $51 \%$, directement ou indirectement, par un ressortissant tanzanien. Certains pensent que la confrontation entre les entreprises de transport maritime et les autorités tanzaniennes a suscité parmi les opérateurs des inquiétudes concernant l'avenir des activités d'agent maritime et a conduit à un retrait des investissements privés et étrangers de ce secteur [Source : The East African on the Web, 3 juin 2002] 
Hutchison Port Holdings (HPH), de Hong Kong Chine, est un grand investisseur, aménageur et exploitant portuaire, qui détient des intérêts dans 15 pays d'Asie, du Moyen-Orient, d'Afrique, d'Europe et des Amériques. HPH exploite un total de 175 postes d'amarrage dans 31 ports ainsi qu'un certain nombre de sociétés de services liés aux transports. La société gère également le port le plus actif du Royaume-Uni (Felixstowe - voir tableau 4). En 2002, HPH a traité 35.8 millions d'EVP. Sa division de logistique sur Internet a été lancée en 2000. HPH fait partie du groupe Hutchison Whampoa, un conglomérat multinational qui a des activités dans 41 pays. Les services portuaires et connexes ne sont que l'un des cinq principaux métiers du groupe [Source: www.hutchison-whampoa.com].

La société PSA, de Singapour, exploite actuellement le plus grand centre mondial de transbordement de conteneurs, qui se trouve dans cette ville. Elle offre à chaque armateur un choix de 200 itinéraires de transport maritime, reliés à 6000 ports de 123 pays. Outre ses activités à Singapour, le groupe PSA participe à 14 projets portuaires dans huit pays, à savoir la Belgique, Brunei, la Chine, l'Inde, l'Italie, la Corée, le Portugal et le Yémen [Source: www.psa.com].

La société philippine International Container Terminal Services s'occupe de gérer, d'exploiter et d'aménager des ports et des terminaux de conteneurs à l'échelle mondiale. En 2001, elle gérait plusieurs ports aux Philippines, six terminaux à l'étranger et prévoyait de développer davantage ses activités internationales. Elle a réalisé récemment le terminal de conteneurs de Suape, au Brésil, et un autre en Pologne, dans la mer Baltique. Elle cherche également à se développer en Asie, au Moyen-Orient et dans les Amériques. En sa qualité d'opérateur portuaire, la société offre divers services de manipulation des cargaisons, dont le chargement, le déchargement et le stockage des cargaisons commerciales; en tant que gestionnaire et aménageur, elle programme, finance et met en œuvre un schéma de développement qui prévoit l'amélioration constante des infrastructures portuaires, des équipements et des techniques informatiques, afin de répondre efficacement aux besoins du commerce international [Source : www.ictsi.com].

La société colombienne Empresa Portuaria Iquique, spécialiste du déchargement et du chargement de cargaisons, exporte jusqu'à $25 \%$ de ses services. Elle a des clients sur tout le continent américain, en Europe (de l'ouest et de l'est), en Asie et au Moyen-Orient. Selon Ronnie Manzo Barriga, son représentant, les installations du port d'lquique sont le point de passage naturel des importations/exportations boliviennes. Compte tenu de cette situation et des accords commerciaux en cours de négociation avec la Bolivie, mais aussi l'Union européenne, les États-Unis et la Corée, on prévoit que les activités portuaires d'Iquique vont connaître un grand essor [Source : étude de l'OCDE].

\section{Services de transport maritime}

127. Des entreprises d'un certain nombre de pays en développement comme le Taipeh chinois, la Chine, Singapour, la Malaisie, le Koweït et la République de Corée ont également acquis un rôle dominant dans le transport maritime sur le plan mondial ; d'autres, d'origine mexicaine, colombienne et chilienne, sont des acteurs importants sur le plan régional. En outre, plusieurs sociétés originaires de pays en développement comptent parmi les principaux opérateurs de services de conteneurs, le critère de classement étant le nombre de bateaux et la capacité totale d'embarquement. Plusieurs facteurs ont contribué à faire de ces entreprises des leaders sur le marché : la formation de co-entreprises régionales pour fournir des types particuliers de services sur des routes sélectionnées du commerce maritime, la concentration sur l'activité intra-régionale ou la fourniture de services annexes en coopération avec les transporteurs internationaux empruntant les principaux itinéraires.

128. Les pays en développement sont également les principaux fournisseurs de main-d'œuvre de la flotte commerciale mondiale. Les Philippines, premier contributeur mondial d'équipages, représentent plus de $20 \%$ du nombre total de marins. On estime que la moitié des équipages de navires battant pavillon des pays membres de l'OCDE sont des ressortissants de pays non membres.

La société Orient Overseas Container Line (OOCL), dont le siège social se trouve à Hong Kong Chine, est un fournisseur international de transport par conteneurs et de logistique. Elle est l'un des principaux transporteurs desservant la Chine, mais offre aussi ses services à toutes les grandes économies commerçantes du monde et relie l'Asie, l'Europe, l'Amérique du nord, la Méditerranée, le sous-continent indien, le Moyen-Orient et l'Australie/NouvelleZélande. OOCL exploite le plus grand porte-conteneurs mondial ( OOCL Shenzhen »), qui a été construit par le groupe coréen Samsung Heavy industries. Neuf autres navires du même type sont en projet, dont le premier (« OOCL Long Beach ») pour livraison en juin 2003 [Source : www.psa.com]. 
Mitsui O.S.K. Lines est une grande compagnie maritime qui recourt à des partenaires des Philippines, du Vietnam et d'Indonésie pour recruter des équipages. L'un de ses fournisseurs de services de main-d'œuvre est la compagnie maritime Magsaysay. Mitsui Lines, fondée en 1948, se livre à de nombreuses activités de services liés au transport maritime comme l'achat de navires, leur gestion, la fourniture des équipages, la formation, les services d'assureur et d'agent maritime. Elle exploite divers types de navires et les équipe en personnel ; elle utilise un effectif total de plus de 17000 personnes, aux fonctions, à l'expertise et aux compétences diverses, dont certaines travaillent à terre et d'autres sont embarquées [Source : étude de l'OCDE].

La société de services de transport international Yansha, de Pékin (société de commerce électronique à longue distance de Pékin), est un expéditeur international de fret spécialisé dans les effets personnels, qui dispose d'une licence délivrée par le ministère chinois du commerce extérieur et de la coopération économique. Créée en 1992, la société compte parmi sa clientèle des invités étrangers, des entreprises et les ambassades étrangères en Chine. Elle offre une très grande variété de services : import/export, réservation d'espace sur les navires, formalités douanières, vérifications, conditionnement professionnel, stockage, assurance, certificats d'antiquité, chargement et déchargement de conteneurs, consultation de fret. Elle dispose d'un réseau d'agents dans certains des plus grands ports du monde. En outre, elle joue le rôle de courtier pour des transactions entre chinois et étrangers portant sur des éléments particuliers, tels que les antiquités chinoises, les entrepôts de meubles anciens et les fabriques, les objets en bronze, la porcelaine, les sculptures en pierre, les tapis et les tableaux [Source: Internet].

Marindolestari Guna est une compagnie de transport maritime, créée en 1984, qui exploite actuellement des barges pour répondre aux besoins de clients indonésiens et de pays voisins. Elle possède une importante flotte de remorqueurs et de barges à grand rayon d'action, qui transporte des cargaisons dans l'ensemble de l'Indonésie, à Singapour et en Malaisie. Couvrant tout l'archipel indonésien, de Sabang à Merauke, elle décharge des cargaisons dans tout le pays. Marindolestari Guna est spécialisée dans le transport d'agrégats, d'asphalte, de charbon, d'équipements lourds et de bûches [Source: Internet].

La compagnie Essar Shipping (Inde) offre des solutions logistiques optimales de transport par mer, grâce à une flotte diversifiée, à des équipements de stockage et à des services de gestion de la chaîne d'approvisionnement. Elle possède 1 milliard d'USD d'actifs et se divise en deux branches : le Groupe de transport d'énergie (ETG) et le Groupe de transport côtier intégré (ICT). Alors qu'ETG fournit des services de gestion de transport maritime à l'industrie mondiale de l'énergie, ICT assure la gestion de la chaîne d'approvisionnement pour le transport par mer de cargaisons en vrac et de produits raffinés. La société dispose d'installations aux États-Unis et en Europe. Au cours de l'année financière 2000-2001, ESL a réalisé un chiffre d'affaires total de 4822 millions d'INR (101 millions d'USD) [Source : Evalueserve] 


\section{SERVICES PROFESSIONNELS}

\section{Vue d'ensemble}

129. Les services professionnels se composent des catégories suivantes: services juridiques, comptables, d'audit et de tenue de livres ; services de conseil fiscal ; services d'architecture, d'ingénierie et connexes ; services médicaux, dentaires, d'infirmerie, paramédicaux et vétérinaires. Cette partie traitera des professions juridiques et comptables ainsi que des services d'architecture, d'ingénierie et connexes. Les services médicaux, dentaires, paramédicaux et d'infirmerie sont abordés dans la partie consacrée aux services de santé.

130. La demande internationale de services aux entreprises et de services professionnels, surtout spécialisés, est très forte, car toutes les organisations - publiques ou privées, à but lucratif ou non lucratif, grandes ou micro-entreprises - ont des fonctions secondaires dont dépendent leur survie et leur compétitivité, mais qui ne sont pas au cœur de leur vocation ou de leur compétence. On peut citer par exemple la comptabilité (dans les entreprises dont ce n'est pas la spécialité), la formation (dans les institutions dont ce n'est pas le métier), la recherche de marchés (idem) et les services informatiques (idem). Aucune organisation, quelle qu'en soit la taille, ne peut perdurer en l'absence de ces fonctions qui améliorent la gestion et la productivité de l'entreprise.

131. Les services professionnels revêtent une importance particulière pour le développement économique, par leur contribution au renforcement des infrastructures (construction, architecture) et à la création d'un environnement favorable à l'investissement et aux entreprises (services juridiques et comptables). Il peut être important d'ouvrir l'accès de ces services au marché pour attirer l'IDE et faciliter le transfert de connaissances.

132. Les pays en développement ont clairement fait part de leur intérêt pour l'exportation de services, pour deux raisons. Premièrement, leurs spécialistes sont concurrentiels sur les marchés internationaux. Deuxièmement, il n'est pas nécessaire d'établir une présence commerciale pour offrir des services professionnels. Cet intérêt est apparent dans plusieurs propositions de négociations et dans les demandes adressées par les pays en développement dans le cadre de la procédure de demandes et d'offres de l'OMC.

133. Les services professionnels ont au moins quatre caractéristiques en commun. En premier lieu, la compétence et la qualité du capital humain sont des facteurs essentiels de réussite. En deuxième lieu, la présence commerciale et les mouvements de personnel restent d'importants modes de fourniture, en dépit des avancées récentes des technologies de l'Internet et des télécommunications. En troisième lieu, la plupart des services professionnels sont soumis à de strictes réglementations internes, pour des raisons de politique publique et de protection du consommateur. Enfin, il y a souvent pénurie de ces services dans les pays en développement.

134. Des recherches effectuées dans les années 1980 en Amérique latine par la CNUCED ont montré que l'un des facteurs critiques de différenciation des pays développés et en développement était la disponibilité de services aux entreprises et de services professionnels de nature spécialisée et de grande qualité. Toutefois, cette situation évolue maintenant. Un nombre croissant de pays en développement sont 
en mesure d'offrir d'excellents services professionnels, grâce à la conjonction d'un capital humain hautement qualifié, d'une technologie de pointe et de prix compétitifs.

135. Le Kenya est un bon exemple de pays où le secteur des services professionnels est bien développé. Il le doit largement aux dépenses publiques consacrées à l'enseignement supérieur et technique, le pays possédant six universités publiques et cinq privées. En 1999, les services professionnels, privés et publics, donnaient du travail à environ 140000 personnes très qualifiées, concentrées surtout dans le domaine médical, l'enseignement, la comptabilité et la gestion.

136. Toutefois, l'activité exportatrice des prestataires de services de pays en développement reste confrontée à de sérieux obstacles. Selon plusieurs entrepreneurs, la principale contrainte qui pèse sur l'exportation de leurs services professionnels est un manque de confiance généralisé des acheteurs potentiels. Une société africaine, qui commercialise maintenant des systèmes de paiement dans les pays de la région et sur les marchés d'Europe et d'Amérique du nord, rapporte que la principale difficulté de départ a été de surmonter le scepticisme des compatriotes et des étrangers quant à la capacité d'entreprises de pays en développement à mettre au point et à exploiter des technologies avancées. De plus, dans l'architecture et l'ingénierie, les préférences des multinationales et l'existence de dispositifs d'aide liée favorisent l'emploi de professionnels étrangers plutôt que locaux. Les restrictions à la délivrance de visas pour les séjours professionnels temporaires, l'absence de reconnaissance mutuelle des références professionnelles et les barrières réglementaires nationales à la fourniture de services professionnels par des étrangers sont perçues comme des obstacles supplémentaires à l'exportation.

\section{Services juridiques}

137. Dans la grande majorité des pays, les métiers du droit sont pratiqués par des professionnels libéraux ou par de petites entreprises, tandis que les grandes sociétés de services juridiques restent un phénomène local, circonscrit à un petit nombre de pays anglo-saxons de droit coutumier. Néanmoins, la mobilité internationale croissante des personnes, des capitaux, des biens et des services, au cours des vingt dernières années, s'est accompagnée du développement d'un marché florissant du conseil juridique international. Un autre facteur de croissance a été l'apparition de nouvelles activités, en particulier dans le domaine du droit commercial. Les restructurations d'entreprises, les privatisations, les fusions-acquisitions internationales, les droits de la propriété intellectuelle et le droit de la concurrence ont engendré une demande de services juridiques de plus en plus sophistiqués. Comme on demande aux juristes une connaissance approfondie du contexte réglementaire où opère une entreprise ainsi que des pratiques et lois en vigueur sur le lieu d'activité, le potentiel de transactions internationales augmente quand il existe des différences sur tous ces plans.

138. Malheureusement, seule une poignée de pays de l'OCDE dispose de données détaillées et exhaustives sur la dimension et les échanges internationaux de ce secteur. La demande de services juridiques émane d'entreprises et d'organisations qui ont besoin d'un courant régulier de conseils juridiques, mais aussi de clients individuels; pour ces derniers, les besoins ne se font sentir qu'occasionnellement et dans des domaines aussi variés que les divorces, les successions, les acquisitions foncières et les questions pénales. Les juristes étrangers, qui offrent des services juridiques transfrontières ou au moyen d'un établissement, jouent surtout le rôle de conseillers juridiques étrangers ; en d'autres termes, ils prodiguent des services de conseil relatifs au droit international, au droit de leur propre pays ou à celui de tout pays tiers pour lequel ils sont qualifiés.

139. Dans la plupart des cas, les exportations des pays en développement s'effectuent par prestation de services transfrontières (mode 1) et, quand le marché de destination le permet, par le déplacement de professionnels libéraux ou de salariés/associés d'une société juridique établie à l'étranger (mode 4). La présence commerciale (mode 3) est relativement peu usitée. Parmi les 135 principales sociétés juridiques 
du monde, seuls trois établissements de pays en développement ont des bureaux à l'étranger : Kim \& Chang (République de Corée), Deacons (Hong Kong) et Pinheiro Neto (Brésil) disposent tous d'une représentation hors de leur pays.

140. L'affiliation à des réseaux internationaux fiables ou l'intégration à des partenariats et une bonne stratégie internationale de communication sont importantes pour des sociétés juridiques petites et moyennes qui cherchent à commercialiser leurs services au niveau mondial.

\begin{abstract}
Mohamed Remmal et Fruhbeck consultants (Maria Elena Pubillones Marin) sont deux sociétés juridiques situées respectivement à Casablanca (Maroc) et à la Havane (Cuba). Malgré leur petite taille, elles commercialisent leurs services à l'étranger grâce à leur affiliation à " International Law firms », une association de cabinets indépendants de droit commercial de toutes les grandes villes du monde, chaque cabinet étant spécialisé dans les domaines importants dans son pays. Ce réseau est fondé sur des relations personnelles entre tous les membres, ce qui permet de recommander en toute confiance les clients à des cabinets associés d'autres pays.
\end{abstract}

Castro Sammartino \& Pierini est une société juridique argentine de taille moyenne, multidisciplinaire, qui a des clients en Argentine et dans le monde entier. Elle est divisée en plusieurs secteurs : services aux entreprises et aux clients institutionnels, services aux clients privés, fiscalité, comptabilité et services financiers, conseils en matière de santé. Au fil des années, elle a tissé un large réseau de relations avec des juristes du monde entier, ce qui lui permet de faire bénéficier les clients des services d'une société juridique internationale, tout en conservant la relation étroite et personnalisée entre clients et professionnels qui caractérise une petite organisation.

En Chine, la société de droit du commerce international Zhejiang offre à sa clientèle des services de conseil juridique relatifs au commerce international, aux projets de coopération économique, à l'exploitation des ressources, aux investissements étrangers et aux questions financières, à l'immobilier, à la fiscalité, au transport maritime international, aux affaires maritimes, au transport aérien, à l'assurance et à l'organisation des sociétés. Elle apporte une assistance à ses clients pour la rédaction, l'examen, la révision et la négociation de contrats, d'accords, de réglementations et d'autres documents juridiques ; elle formule aussi des opinions juridiques dans le cadre de services d'arbitrage et de solution de litiges; enfin, elle contribue à la médiation ou à la solution de différends nés dans le contexte d'affaires internationales.

\title{
III. Services de comptabilité, d'audit et de fiscalité
}

141. Les auditeurs ont pour fonction à garantir aux utilisateurs d'états financiers que les faits rapportés sont exacts et d'attirer l'attention sur tout problème relatif à ces états ou sur la situation financière de la société, indépendamment de la conformité aux normes. Comme la plupart des utilisateurs ne comprennent guère comment les normes de comptabilité et d'audit sont appliquées, ils considèrent probablement les états financiers comme crédibles s'ils sont établis par des experts comptables réputés. Le besoin de crédibilité et l'augmentation des coûts de transaction dans un secteur très gourmand en capitaux ont abouti à la domination du marché mondial par une poignée de sociétés communément appelées les "Quatre Grandes ». Pourtant, le travail est souvent effectué par des «entreprises affiliées », qui bénéficient de la réputation résultant de leur rattachement à un acteur de premier plan, tout en offrant des audits d'un bon rapport coût/efficacité aux branches locales de leurs clients multinationaux. Ainsi, en Europe de l'est, l'audit des rapports financiers est effectué par des sociétés locales affiliées aux Quatre Grandes.

142. Le marché mondial des services comptables croît et change rapidement. Le commerce électronique, la libéralisation des échanges et la fixation d'un ensemble de principes comptables internationaux communément acceptés ont ouvert les marchés nationaux à la concurrence étrangère et contribué à élargir le champ d'action des professions liées à la comptabilité. En outre, les consommateurs de services fiscaux, d'audit et comptables traditionnels sont de plus en plus demandeurs de solutions financières globales. En conséquence, cette discipline s'est éloignée de sa base étroite d'audit et de conseil fiscal pour embrasser tout un ensemble de services comme la finance d'entreprise, le redressement et la 
solvabilisation de sociétés, la remise en ordre des comptes à la suite de contentieux et la sécurité des entreprises.

143. Toutefois, au fur et à mesure que les ventes croisées de services de conseil aux entreprises et d'autres services d'assurance sont devenues plus fréquentes, l'indépendance des commissaires aux comptes a été entamée. De sérieux conflits d'intérêts sont apparus, comme on l'a vu avec l'affaire EnronAndersen et d'autres cas similaires (Waste Management, Cendant, MicroStrategy, Xerox). Dans ces conditions, les firmes d'expertise comptable se défont de leurs activités non essentielles et concentrent leurs ressources sur les fonctions les plus importantes, tout en sous-traitant d'autres domaines. La soustraitance des processus d'entreprise (Business Process Outsourcing - BPO) consiste soit à transférer une fonction ou un service, soit à déléguer des responsabilités quotidiennes non fiduciaires à un tiers fournisseur. Cela permet d'obtenir les meilleures pratiques à un coût parfois très inférieur à celui d'une réalisation au sein de l'entreprise. La révolution technologique de la dernière décennie a rendu la soustraitance plus efficace et accessible que jamais, tout en améliorant sensiblement le contrôle et la qualité des fonctions déléguées. On a prévu que la BPO se développerait à une vitesse spectaculaire ; selon Gartner Dataquest, elle dépassera 38 milliards d'USD en 2004 dans les services financiers.

Les opérations communément sous-traitées comprennent : la tenue de la comptabilité pour les clients, les solutions de conseil fiscal, la gestion de document, les services de mise à disposition de personnel et de technologies de l'information. Kishore Mirchandani est directeur général d'itAccounts, une " société de sous-traitance de processus d'entreprise », qui fournit des services de comptabilité, d'exécution d'opérations fiscales et d'imagerie documentaire ; elle est installée à Bangalore, dans l'état indien de Karnataka. Dans la grande majorité des cas, les exportations de cette nature s'effectuent par passage de frontières (mode 1), les documents et les conseils étant transmis par les canaux traditionnels de la poste ou par courrier électronique. L'évolution des TIC permet de livrer des services au-delà des frontières selon des modalités de plus en plus efficaces et accessibles. Le mouvement de professionnels libéraux ou de salariés/associés d'une entreprise établie à l'étranger (mode 4) est également fréquent.

Les entreprises qui ne sont pas affiliées à une des " quatre grandes » peuvent adhérer à la Conférence des praticiens de la comptabilité affiliés (Affiliated Conference of Practicing Accountants - ACPA), un réseau mondial de 80 firmes comptables indépendantes de 35 pays des 5 continents, qui se recommandent mutuellement. En Amérique du Sud, les sociétés affiliées sont Senso Auditores Indipendentes (Brésil), UC\&CS (Mexique), Flores Konja y Asociados Contadores Publicos S.C. (Pérou), et Briner \& Asociados (Venezuela). En Afrique, les principales références sont la société égyptienne Talaat I. Hanna \& Associates et la société kényane Aswin Brothers. Le MoyenOrient et le sud de la Méditerranée sont représentés par la société chypriote Constantinou \& Kyriacou, par Hogan, Ginzburg Anvi \& Co. en Israël, par la société comptable et de conseil financier Vizyon en Turquie et par la société d'expertise comptable Puthran à Dubaï. Enfin, les sociétés asiatiques affiliées sont Sunil Goel \& Associates (Inde), Salaki \& Salaki (Indonésie) et First United CPA Office (Taipeh chinois).

\section{Services d'architecture et d'ingénierie}

144. Les services d'architecture comprennent le conseil en matière d'assurance, l'avant-projet, le projet, la gestion des contrats, l'aménagement urbain et l'architecture paysagère. Les services d' « ingénierie » se composent de la conception et du conseil en matière d'édification des fondations et des structures de bâtiments, d'installations mécaniques et électriques dans les constructions, de travaux de génie civil, de processus et production industriels. Dans la grande majorité des pays, ces deux secteurs de services sont relativement fermés aux fournisseurs étrangers.

145. Les améliorations de la gestion et de l'organisation, conjuguées aux progrès notables de la formation d'ingénieurs et d'architectes dotés de compétences et d'expertises spécifiques, ont contribué à multiplier le nombre de professionnels originaires de pays en développement. Ce facteur est primordial, car les échanges transfrontières de services d'architecture et d'ingénierie tendent à augmenter parallèlement à la complexité et à la spécialisation des services concernés.

146. En outre, les liens réciproques que les services d'architecture et d'ingénierie établissent entre différents secteurs de l'économie - particulièrement entre les unités de production, les activités locales de 
R\&D, la fabrication de biens de capital et les services financiers - sont éminemment importants. Par conséquent, les pays en développement qui disposent de capacités en ce domaine sont mieux en mesure de faire acte de candidature pour l'exécution de projets de construction sur leur marché et à l'international.

147. Quinze sociétés de pays en développement font partie des 151 premières entreprises mondiales de conception (en architecture et ingénierie), classées en fonction du chiffre d'affaires réalisé dans l'exécution de projets à l'étranger. La mieux placée est une société égyptienne, qui occupe le douzième rang mondial, tandis que quatre autres entreprises de pays en développement, originaires respectivement de Chine, du Liban, d'Israël et de Corée, figurent dans les 100 premières. Parmi celles qui sont classées de $100^{\mathrm{e}}$ à $151^{\mathrm{e}}$, on trouve sept entreprises chinoises, une taïwanaise, une sud-africaine et une yougoslave. (voir tableau A.4 en Annexe, Partie I).

Dar Al-Handasah (Shair and Partners) est un bureau d'études international, spécialiste de l'architecture, de l'ingénierie, de l'aménagement, de l'environnement et de l'économie. Fondée en 1956, la société est aujourd'hui au premier rang mondial en matière de conception et d'ingénierie (voir tableau A1 à l'Annexe de la Partie I). Surtout active à Londres, au Caire et à Beyrouth, Dar emploie 4000 professionnels répartis dans une cinquantaine de bureaux en Afrique, au Moyen-Orient et en Europe.

Un courant d'échanges existe aussi entre le Brésil et d'autres pays en développement, notamment la Chine et l'Inde. La Société brésilienne de conception, d'ingénierie et de construction a ouvert un bureau de représentation à Pékin et, dans le cadre du développement de la sous-traitance de main-d'œuvre chinoise au Brésil, 8 projets de contrats d'ingénierie, d'un montant de 18.41 millions d'USD, ont été lancés jusqu'à la fin de 1999. Une société brésilienne de conseil en projets énergétiques, M/s Enerconsult S.A., a monté une co-entreprise avec l'entreprise indienne Tata Consulting Engineers, installée à Sao Paulo, pour fournir des services dans ce domaine.

148. Les services de conseil spécialisés en ingénierie représentent une importante activité d'exportation pour l'Inde, d'abord à destination de l'Afrique et de l'Asie, ensuite du Moyen-Orient et dernièrement d'autres marchés. La plupart des consultants indiens en ingénierie s'occupent d'aspects touchant à l'environnement et à l'énergie. L'avantage comparatif avéré de l'Inde dans ce secteur a incité le gouvernement à ouvrir le marché national à la concurrence, en supprimant les contrôles sur la participation de capitaux étrangers et en étendant le traitement national intégral aux firmes étrangères commercialisant leurs services en Inde [Source : CNUCED].

149. Les architectes malaisiens ont conçu des lieux de vacances, des hôtels, des immeubles d'habitation et de bureaux, des usines et des zones résidentielles dans des pays comme le Vietnam, les Philippines, la Namibie, l'Afrique du Sud et l'Australie [Source : MATRADE - Société de développement du commerce extérieur malaisien]. 


\section{SERVICES DE TÉLÉCOMMUNICATIONS}

\section{Vue d'ensemble}

150. Avec les progrès de la technologie, il est devenu plus important pour l'ensemble de l'économie de disposer de services de télécommunications modernes et efficaces. Ils jouent en effet un rôle fondamental pour les échanges de beaucoup d'autres services, notamment plusieurs de ceux que cette étude a distingués comme services aux entreprises, professionnels et d'enseignement. De nombreux pays, reconnaissant le rôle essentiel des télécommunications et recherchant des investissements pour développer les infrastructures nécessaires et les technologies de pointe, ont entrepris une libéralisation notable de ce secteur. Le résultat est une industrie fortement internationalisée, dont les principaux acteurs - souvent originaires de pays de l'OCDE - opèrent sur une série de marchés dans le monde par l'intermédiaire de filiales contrôlées intégralement, de co-entreprises et de partenariats. Bien que nombre de leaders mondiaux des télécommunications appartiennent aux pays de l'OCDE, certaines entreprises de pays en développement jouent un rôle significatif sur un plan régional et, parfois, international.

151. Les exemples qui suivent de sociétés de pays en développement qui exportent des services de télécommunications sont divisés par régions, plutôt que par nature de service, et cela pour deux raisons. En premier lieu, la plupart des entreprises citées ci-dessous offrent une large gamme de services de télécommunications, allant de la téléphonie de base et mobile aux derniers services à bande large. En deuxième lieu, il ressort de ces exemples initiaux que beaucoup de sociétés de pays en développement se livrent principalement au commerce intra-régional : celles d'Amérique latine alimentent surtout le marché latino-américain, tandis que celles d'Égypte et d'Afrique du Sud exportent vers les autres pays du continent africain. Bien qu'elles s'impliquent également dans les échanges régionaux, les sociétés asiatiques semblent avoir plus d'activités à l'échelle mondiale ; il est vrai que, dans certains cas, cela peut s'expliquer par les différents types de services fournis.

\section{Afrique}

152. Le phénomène de la téléphonie mobile en Afrique présente la particularité d'être conduit par des entreprises africaines. Les grands groupes étrangers, comme Vodaphone ou le groupe français Orange, ont hésité à investir depuis la crise qu'a connue le secteur des télécommunications en 2000 [Source: "How Africa joined the wireless world”, Financial Times, 27 novembre 2003].

MSI Cellular, entreprise enregistrée aux Pays-Bas, dirigée par le Soudanais Mohamed lbrahim et plus connue sous la marque Celtel, est présente dans 12 pays d'Afrique [Source: "How Africa joined the wireless world", Financial Times, 27 novembre 2003].

Econet, créée par l'entrepreneur du Zimbabwe Strive Masiyiwa et seule entreprise de technologie cotée à la Bourse de Harare, possède des intérêts à la fois en Afrique - elle s'est implantée récemment au Kenya - et ailleurs, notamment en Nouvelle-Zélande où elle propose un service de mobile GSM [Source: "How Africa joined the wireless world", Financial Times, 27 novembre 2003]. 
Orascom Telecom, société égyptienne dont les activités sont maintenant concentrées en Afrique du Nord, était à une époque le plus grand opérateur de téléphonie mobile GSM au Moyen-Orient, en Afrique et dans le sous-continent indien; elle disposait alors de 21 licences dans la région. En 2000, elle a acquis Telecel, un groupe africain établi à l'origine au Congo. Au plus fort de son expansion, Orascom Telecom gérait des réseaux GSM dans de nombreux pays du Moyen-Orient et d'Afrique : Algérie, Bénin, Burkina Faso, Burundi, Congo, Côte d'Ivoire, Égypte, Gabon, Jordanie, Niger, Ouganda, Pakistan, République centrafricaine, République démocratique du Congo, Syrie, Tchad, Togo, Tunisie, Yémen, Zambie et Zimbabwe. En janvier 2002, la société et ses filiales GSM comptaient plus de 6757 salariés. Depuis, elle a cependant revendu une grande partie de ses intérêts pour diminuer ses dettes.

Outre ses activités en téléphonie mobile, Orascom Telecom possède d'autres filiales dans les domaines de l'Internet et des technologies satellitaires. La filiale Internet LINKdotNET est le principal fournisseur d'accès à Internet égyptien et elle est associée à MSN Arabie, le premier portail international en arabe et en anglais du monde arabe. LINKdotNET a des bureaux régionaux à Dubaï, dans les Émirats et en Jordanie. Les deux filiales de services par satellite fournissent des équipements et des liaisons satellitaires dans la totalité du Moyen-Orient et des marchés africains : Egyptian Space Communications s'occupe de la conception, de l'installation et de la gestion des liens de communication par satellite, tandis que M-Link offre des services d'entrée pour acheminer le trafic téléphonique en direction et en provenance de l'Afrique [Source : www.orascomtelecom.com et "How Africa joined the wireless world", Financial Times, 27 novembre 2003].

Le secteur sud-africain de la téléphonie mobile comprend Vodacom (contrôlé par le groupe semi-public Telkom et par Vodafone en tant que partenaire minoritaire) et son concurrent MTN. Ils regroupent à eux deux plus de 15 millions d'utilisateurs de téléphones mobiles et ne se limitent pas au marché intérieur. MTN a dépensé jusqu'à présent 600 millions d'USD en dehors de l'Afrique du Sud et recherche de nouveaux débouchés sur le continent africain. Elle a notamment dépensé 285 millions d'USD en 2001 pour obtenir une licence au Nigeria [Source: "How Africa joined the wireless world", Financial Times, 27 novembre 2003].

South Africa's MTN, lancée en 1994, possède l'un des plus grands réseaux GSM du monde, auquel $94.5 \%$ de la population de l'Afrique du Sud a accès. En outre, MTN s'est rapidement développée dans l'ensemble du continent africain, en s'installant au Cameroun, au Rwanda, au Swaziland, en Ouganda et au Nigeria [Source : www.mtn.co.za ; www.cellular.com].

MTN Cameroun couvre 8 des 10 provinces du pays au moyen de 66 stations opérationnelles. La société proclame son engagement en faveur de la formation professionnelle et technique, à la fois interne et externe. MTN Swaziland couvre $75 \%$ du pays et emploie 72 salariés. Elle a presque doublé la taille des marchés de télécommunications nationaux et s'attribue largement le mérite d'une augmentation de la télédensité de près de $7 \%$. MTN a consacré 285 millions d'USD à l'achat d'une licence GSM au Nigeria, ce qui représente son plus gros investissement en dehors d'Afrique du Sud. MTN Nigeria a maintenant 453 cellules sur 173 sites radio, à quoi s'ajoutent 55 sites de transmission. Selon certaines informations [Source : Lagos Vanguard, 26/12/2002, sur www.allafrica.com], les investissements de MTN au Nigeria ont incité d'autres sociétés sud-africaines (comme Eksom et SAA) à envisager des opérations similaires. MTN Ouganda contrôle actuellement $65 \%$ du marché total des télécommunications et $83 \%$ de celui de la téléphonie mobile. Conformément à ses obligations de service public, MTN Publicom (une filiale) a installé 1800 téléphones publics payants dans l'ensemble du pays. MTN RwandaCell, établie en 1998, couvre les 11 principales régions du Rwanda et emploie 30 salariés d'origine locale. Conformément à la philosophie de MTN en faveur de la participation d'éléments locaux, MTN Rwanda a donné à des entreprises locales un rôle crucial dans l'extension de son réseau de distribution; elle dispose de plus de 70 points de vente dans l'ensemble du pays.

Selon le site Internet de MTN, ses filiales entretiennent des relations avec les communautés des pays hôtes. MTN Rwanda est engagée dans des actions communes de parrainage avec des services d'urgence, un orphelinat, des associations de lutte pour la sécurité routière et la protection des gorilles; elle soutient aussi financièrement l'équipe nationale de football. MTN Ouganda a consacré plus de 1 milliard d'UGX à divers projets communautaires et humanitaires. MTN Swaziland a également financé pendant un an le salaire d'un conseiller du groupe national d'action contre les mauvais traitements et a servi de sponsor officiel de l'Association du Swaziland pour les jeux olympiques et les jeux du Commonwealth. 


\section{Asie/Asie du Sud}

Les filiales de Telecom Malaisie offrent des services de télécommunications mobiles au Bangladesh, au Cambodge et au Sri Lanka ; des services de télécommunications et connexes en République de Guinée ; des services de télécommunications au Malawi. Des sociétés associées fournissent des services de télécommunications et connexes au Ghana, des services de télécommunications en Afrique du Sud, des services de télécommunications et de radiotélédiffusion en Thaïlande, enfin des services de transmission radio mobile au Cambodge [Source: www.telekom.com.my].

Hutchison Telecommunications (Hong Kong Chine) assure à l'échelle mondiale un grand éventail de services de télécommunications intégrés et est l'un des principaux opérateurs mondiaux de communications mobiles. En dehors de Hong Kong, l'activité internationale du groupe se déploie en Europe (où il a édifié et ensuite vendu le réseau PCS d'Orange), en Australie, Israël, Inde, Thaïlande, Malaisie, Sri Lanka, Paraguay, Argentine et Ghana. Le groupe s'est engagé activement pour l'obtention de licences de $3^{\text {ème }}$ génération au Royaume-Uni (dans le cadre d'une co-entreprise avec NTT DoCoMo et KPN) ; il a fait de même en Irlande, Italie, Suède, Autriche, au Danemark et en Israël, par l'intermédiaire de filiales. La filiale de télécommunications d'Hutchison en Australie s'est fait également attribuer une licence de $3^{\text {ème }}$ génération dans ce pays et a noué une alliance stratégique avec Nouvelle-Zélande Telecom pour fournir les services correspondants [Source : www.hutchison-whampoa.com].

Hughes Software Systems (HSS) est une entreprise indienne qui fait partie de la société Hughes Electronic. Fondée en 1992, HSS propose à ses clients des services de sous-traitance, qui vont de " la conception et de la mise au point de nouveaux produits » à " l'ingénierie et aux tests » dans divers domaines. II s'agit de réseaux sans fil, de gestion de réseau, de mise en réseau optique, de systèmes de commutation, de réseaux convergents et à bande large, etc. HSS offre également des produits pour transmission d'informations à satellite ( Voice over Packet ») et pour des " données mobiles » ainsi que des solutions d'intégration de nouvelles technologies (« Legacy solutions »). La société a plus de 180 clients dans le monde entier et emploie environ 1600 personnes. Elle possède des bureaux aux ÉtatsUnis, au Royaume-Uni, en Allemagne et en Inde. Elle est représentée par des canaux de distribution en Chine, à Taïwan, en Corée du Sud, au Japon, en Australie, en Nouvelle-Zélande et au Brésil. Ses centres de développement sont situés en Inde, à New Delhi et à Bangalore [Source : Evalueserve].

La société Packetware India, créée en 1996, emploie quelque 50 personnes hautement qualifiées et réalise un chiffre d'affaires d'environ 15 millions d'INR. Elle fournit des logiciels de télécommunications et des services à une clientèle située aux États-Unis, en Europe et en Asie-Pacifique. La société offre, à partir de ses bureaux à l'étranger et du centre de développement indien d'Hyderabad, des services de développement extra-territorial et d'entretien, la mise au point d'applications pour les télécommunications et des conseils sur ses sites avec un bon rapport coût/efficacité [Source : Evalueserve].

La société Asia Satellite Telecommunications, de Hong Kong, est un producteur moyen de capacité de transpondeur satellitaire pour la radio-télédiffusion et les télécommunications. Elle est très tournée vers les marchés étrangers et a des clients sur tous les continents, à l'exception de l'Afrique. En Asie, elle exporte vers la Corée du Sud, le Myanmar, la Malaisie, Singapour, le Vietnam et le Pakistan ; au Moyen-Orient vers le Koweït et l'Arabie Saoudite ; en Europe vers la France, l'Allemagne et le Portugal ; en Amérique du nord vers les États-Unis ; et en Océanie vers l'Australie. Compte tenu de la surcapacité qui existe dans ce secteur et de la dure concurrence des autres producteurs, elle ne prévoit pas de croissance de ses activités [Source : étude de l'OCDE].

L'entreprise indienne Data Access a créé un réseau mondial d'opérateurs de téléphonie et de transmission de données entre l'Asie du Sud, l'Europe et les États-Unis. En Inde, elle a mis en place un réseau comportant cinq centres (situés à Delhi, Mumbai, Bangalore, Chennai et Kolkata) et de nombreux points d'interconnexion avec d'autres réseaux d'opérateurs [Source : Commission européenne].

Reliance Infocom (Inde) a annoncé en octobre 2003 son intention d'acquérir FLAG Telecom, fournisseur international de communications et d'infrastructures de réseaux de télécommunications, pour un montant global de 207 millions d'USD. D'après M. Anil Ambani, vice-président et directeur général de Reliance Industries, il s'agit de la première acquisition internationale de Reliance, et de la plus importante acquisition internationale effectuée par une entreprise indienne dans le secteur des services. FLAG possède un réseau sous-marin de câbles en fibre optique de 50000 kilomètres qui complétera le réseau numérique de $3^{\text {ème }}$ génération de Reliance Infocom. [Source; www.thehindubusinessline.com]. 


\section{Amérique latine}

América Móvil, la division de téléphonie mobile de l'ancien monopole d'État mexicain et leader des télécommunications Teléfonos de México (Telmex), a acquis des licences au Brésil et étendu ses activités dans des pays d'Amérique centrale et dans la partie nord des Andes. La société déclare 30.7 millions d'abonnés en Argentine, en Colombie, au Brésil, en Equateur, au Guatemala et au Mexique (du fait de ses participations), ce qui en fait le numéro 1 de la région devant la société espagnole Telefónica. América movil compte 20 millions d'abonnés au Mexique. En 2002, leur nombre a augmenté de $18 \%$ sur le marché national et de $37 \%$ dans l'ensemble de la région [Source : LatinTrade].

Impsat Fiber Networks, société argentine de télécommunications, est un fournisseur important de données à large bande passante totalement intégrées ainsi que de services Internet et de télécommunications vocales en Amérique latine. Impsat possède et exploite un réseau à large bande de forte capacité dans l'ensemble de l'Amérique latine, en utilisant les technologies les plus évoluées. La société offre des services de télécommunications intégrés à plus de 3 000 clients : entreprises nationales, multinationales et entités publiques; elle fournit aussi des services de gros à des opérateurs fournisseurs d'accès à Internet et à d'autres fournisseurs de services dans l'ensemble de la région. Mis à part l'Argentine, Impsat est installée en Colombie, au Brésil, au Venezuela, en Equateur, au Mexique, au Chili, au Pérou et aux États-Unis. En 2000, les services de réseau représentaient $66 \%$ du chiffre d'affaires, Internet $11 \%$, les projets clé en main $18 \%$ et les services de télécommunications $5 \%$ [Source : www.impsat.com; http//:biz.yahoo.com; www.business.com].

Au Chili, le secteur des télécommunications était caractérisé au début des années 80 par une pénurie de lignes téléphoniques et de fonds pour développer les infrastructures nécessaires. Dix ans plus tard, l'État, tout en renonçant complètement à ses participations, a ouvert ce secteur aux investissements privés nationaux et étrangers. A la suite de la libéralisation, les technologies de pointe ont fait leur entrée dans le pays, notamment les réseaux numérisés, les fibres optiques et les équipements satellitaires. Grâce à sa compétitivité-prix et à des infrastructures efficaces, le Chili a pu passer des accords avec le Pérou et l'Argentine pour fournir à la région frontalière des services de télécommunications; cette première expérience d'exportation régionale a constitué une bonne base pour commercialiser ensuite des services de télécommunications sur les marchés internationaux [Source : CNUCED].

La société chilienne ENTEL fournit des services dans de nombreux pays d'Amérique latine et aux États-Unis. Sa filiale Americatel est présente au Pérou, au Venezuela et en Amérique centrale sur le marché des appels longue distance intérieurs et internationaux. Le chiffre d'affaires réalisé par l'intermédiaire d'Americatel représente $30 \%$ du chiffre d'affaires total d'ENTEL. Au Pérou et au Venezuela, la société détient des licences de télécommunications sans fil [Source : Commission européenne]. 


\section{SERVICES RELATIFS AU TOURISME ET AUX VOYAGES}

153. Les agences de voyage, les voyagistes, les guides touristiques ainsi que les hôtels et restaurants sont tous des exportateurs importants de services dans les pays en développement, créant des emplois et faisant entrer des devises étrangères. Les organismes de commercialisation des destinations - qui se composent souvent de partenariats entre les divers prestataires de services (compagnies aériennes, hôtels, organisateurs d'attractions, voyagistes) et administrations publiques compétentes - jouent un rôle croissant dans l'industrie mondiale du tourisme; ils aident les destinations à promouvoir leurs "produits d'information », à se faire une place sur le marché et à attirer des clients. Ils peuvent être un facteur crucial de la réussite touristique des pays en développement, dans la mesure où ils permettent à l'industrie nationale de créer de nouveaux canaux de distribution et de vente.

154. Étant donné l'importance du tourisme pour les pays en développement, l'objectif de cet article n'est pas de donner des exemples tirés de tous ceux qui sont fortement engagés dans le tourisme. L'accent est plutôt mis sur trois domaines. En premier lieu, on présente l'exemple d'un pays qui a abondamment répondu à l'enquête, afin de montrer la gamme des activités susceptibles d'être concernées. Ce pays, la Colombie, fait face à de graves problèmes internes, mais, si certains opérateurs ne sont pas optimistes, d'autres croient au développement du tourisme. Un cas retient particulièrement l'attention : une société colombienne a exporté un concept touristique (les parcs à thème agricole) dans d'autres pays; soit cette société investit directement, soit elle vend le concept ou encore intervient comme consultant. En deuxième lieu, on examine comment les pays en développement exploitent des créneaux ou de nouveaux types de tourisme, tels que l'écotourisme. En troisième lieu, on donne des exemples de tourisme sur Internet (portails et services de réservation), un domaine dans lequel le commerce électronique a un gros potentiel dans les pays en développement.

Expreso Viajes y Turismo - American Express est une société colombienne de 120 salariés, qui travaille dans le secteur des services de voyage. Son principal marché d'exportation est les États-Unis, avec environ $10 \%$ du total. Toutefois, le propriétaire de la société estime que les perspectives du tourisme sont mauvaises en raison de la situation très difficile du pays dans un contexte de violence et guérilla [Source : étude de l'OCDE].

En revanche, l'hôtel Casa Madrigal, un hôtel colombien de taille moyenne spécialisé dans les réceptions organisées pour des institutions et des sociétés, s'attend à une expansion de son activité internationale due surtout à la dollarisation du voisin équatorien, à l'abaissement des droits de douane et à des prix compétitifs. Une autre entreprise colombienne, La Casona del Patio Amarillo, partenaire d'une société britannique dotée d'un système mondial de réservation, prévoit d'attirer plus de touristes étrangers au moyen d'une intense activité promotionnelle ciblant des portails Internet. [Source : étude de l'OCDE].

Turismo Abordo est une agence de voyage qui fait la promotion du tourisme dans la capitale colombienne, Bogota, et dont $25 \%$ des clients viennent d'Espagne, de Grèce et de France. Elle travaille aussi pour des succursales de sociétés étrangères installées en Colombie comme Pronet, Alborada Venezuela et Monigramm Gracia. Turismo Abordo prévoit d'augmenter sa clientèle internationale grâce à ses contacts dans le monde et à sa participation aux foires et manifestations internationales. Si l'on en croit son propriétaire, les possibilités d'expansion dans ce secteur se trouvent surtout dans le tourisme de loisir et les voyages d'affaires [Source : étude de l'OCDE]. 
Hacienda El Eden Resort est une station touristique de taille moyenne, spécialiste de la mutltipropriété et située dans une région de la Colombie qui se prête particulièrement à l'écotourisme et au tourisme sportif, la Valle del Cauca. Le propriétaire, Mario Cifuentes Ortiz, estime que son affiliation à des réseaux internationaux de multipropriété ainsi que ses partenariats avec des sociétés et des stations touristiques d'Equateur, du Pérou, du Venezuela et des États-Unis sont à l'origine de 25 à $50 \%$ du chiffre d'affaires total. M. Ortiz anticipe une progression de la part internationale de son activité sous l'influence de plusieurs facteurs : l'essor général du secteur de l'écotourisme, les débouchés offerts par la multipropriété en Colombie, les tarifs compétitifs qu'il est en mesure de proposer et le succès croissant du sudouest du pays. "Une amélioration de l'accès au financement et des connexions téléphoniques donnerait une impulsion supplémentaire à la profession » affirme-t-il [Source : étude de l'OCDE].

Panaca (Colombie) est une société importante, d'un effectif dépassant 250 personnes, qui englobe un parc à thème agricole, un espace culturel et hôtelier ainsi qu'une fondation. Elle propose des services interactifs à caractère éducatif, culturel, commercial et de conseil dans les domaines de l'agriculture et de l'agro-tourisme. La société attire une clientèle colombienne et étrangère avec l'organisation en 2003 de plus de 20 manifestations, qui vont des foires animalières et des concours de beauté pour animaux domestiques aux journées éducatives consacrées à des sujets comme le développement viable et les traditions locales. Aux dires de son représentant, M. Novoa, la base de clientèle internationale, qui contribue à plus de $10 \%$ du chiffre d'affaires total, devrait augmenter à l'avenir. En outre, les dirigeants de la société sont en train d'exporter le concept dans d'autres pays, soit en investissant directement dans de nouveaux parcs, soit en vendant leurs services de consultants aux investisseurs locaux. Au Mexique, ils ont déjà souscrit à un dispositif d'exonération fiscale pour la création d'un parc dans la région rurale autour de la ville de Reinosa, proche de la frontière avec les États-Unis. Après l'ouverture, prévue au premier trimestre 2004, trois parcs supplémentaires verront le jour dans d'autres régions du Mexique (un à Mexico, le deuxième dans la zone comprise entre Guadalajara et Querétaro, le troisième à San Miguel Allende). Un autre parc à thème est programmé au Costa Rica dans la région d'Orotina, au sein d'un grand parc national ; en Espagne, la société va chercher un emplacement proche de Madrid [Source : étude de l'OCDE].

Travel Depot Representaciones est une entreprise colombienne qui vend des services touristiques aux États-Unis, aux Caraïbes et en Amérique du Sud. Son représentant, Juan Carlos Camacho, prévoit une croissance de la part internationale de l'activité, qui représente actuellement $75 \%$ du total. II estime que la baisse des tarifs de transport aérien et du coût des voyages ont beaucoup contribué à cette croissance, au même titre que l'attrait des destinations latino-américaines [Source: étude de l'OCDE].

155. Ces deux dernières décennies, une nouvelle forme de tourisme est devenue de plus en plus à la mode, l'écotourisme. Plusieurs pays en développement ont commencé à faire la promotion de leur patrimoine environnemental préservé et de leur diversité écologique, afin d'attirer ceux qui recherchent des solutions de remplacement au tourisme de masse et aux voyages organisés. En Tanzanie, la contribution du tourisme au PIB est aujourd'hui de $14 \%$ et les entrées de devises à ce titre ont atteint 725 millions d'USD en 2001, contre 259.4 millions d'USD en 1995. Cette augmentation s'explique en partie par le succès de nouveaux produits touristiques comme l'écotourisme (qui tire parti des vastes parcs nationaux et de la grande biodiversité du pays) et le tourisme de plage [PricewaterhouseCoopers (2003)].

A Cuba, les circuits écologiques « au-delà des plages » ont été conçus pour permettre aux touristes de découvrir l'environnement naturel exceptionnel de l'est du pays. Organisés à partir de Santiago de Cuba, ville située entre les Caraïbes et la Sierra Maestras, les tours suivent la côte et pénètrent dans les montagnes pour explorer certains des micro-climats locaux. L'accompagnement est toujours assuré par des traducteurs expérimentés et un personnel bilingue. On offre aux clients des logements choisis avec soin (chez l'habitant, dans des éco-stations et des hôtels 3 à 5 étoiles), le transport avec air conditionné et un forfait global [Source : Cubanet].

Depuis 30 ans, TOURINDIA fait la promotion du tourisme dans l'État de Kérala, au sud de l'Inde. Son directeur général, Babu Varghese, a lancé Tourindia sous forme d'office de tourisme au début des années 1970, époque où cette activité n'en était qu'à ses premiers pas. Pour répondre à la demande naissante d'un tourisme "vert », il a inventé une formule de croisières confortables sur les canaux locaux en transformant les cargos traditionnels du Kérala, appelés "Kettuvallam », en bateaux de plaisance du même nom. Parmi les initiatives ultérieures d'écotourisme, on peut citer tout d'abord " la maison dans les arbres dans un cadre naturel magique » : il s'agit d'une véritable expérience de vie en forêt dont l'incidence sur l'environnement est minimale, puisque tout le nécessaire doit être emporté, les touristes n'utilisant que des matériaux locaux et des techniques indigènes; il existe également " la piste du tigre de Periyar », une randonnée aventurière à vocation protectrice de l'espèce à laquelle participent d'anciens braconniers [Source : ecotourism2002.org]. 


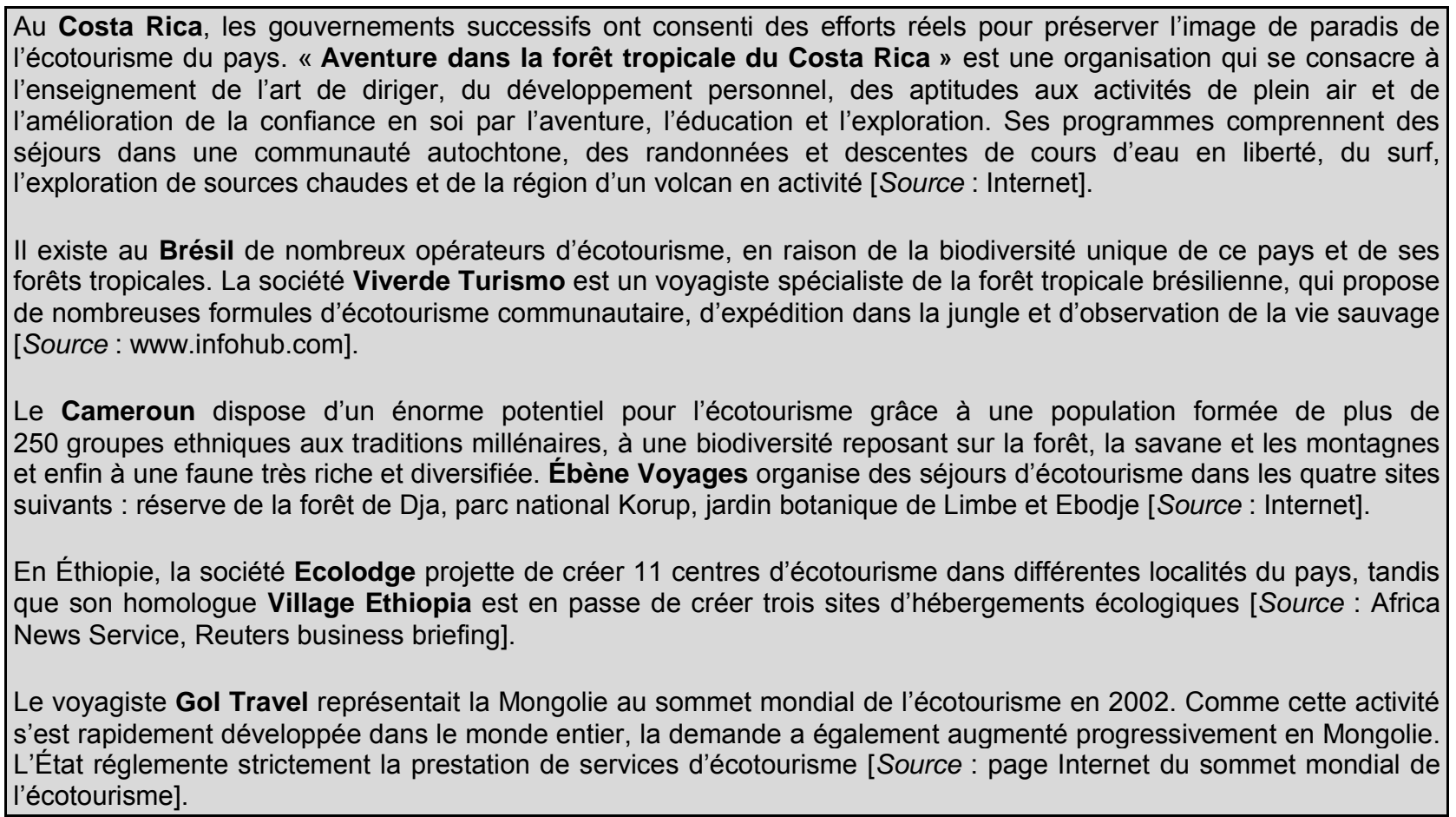

156. Le tourisme électronique est un autre domaine prometteur pour l'exportation et l'un des secteurs du commerce électronique dont la croissance est la plus rapide. La CNUCED (2001) rapporte que la main-d'œuvre compétente, moderne et initiée au numérique des pays en développement jouit d'un avantage comparatif dans le tourisme par Internet, qui, au point de vente, est largement un produit d'information. Selon cette organisation, les réservations de voyages par Internet ont presque doublé en 2000 aux États-Unis et en Europe, pour atteindre 15.5 milliards d'USD ; ce montant dépasse celui des achats électroniques de logiciels et matériel informatique, qui étaient auparavant la principale catégorie de transactions. Sur les 64 milliards d'USD de chiffre d'affaires du commerce électronique réalisé en 1999 dans les pays développés, les réservations de voyages, de transports et d'hôtels prises en bloc ont représenté, avec $38.5 \%$ du total, la proportion la plus importante.

157. La CNUCED estime que, dans les pays en développement, le tourisme représente au moins la moitié des activités de commerce électronique, dont il constitue la branche la plus importante. Ce marché potentiel est évalué à 5 milliards d'USD, mais ils ne semblent pas actuellement en mesure d'en capter plus de $10 \%$, en raison surtout du manque d'ordinateurs, de la pénétration insuffisante d'Internet et du manque de matériel de paiement électronique par carte de crédit.

158. Toutefois, le fait que la plupart des consommateurs de produits touristiques se trouvent dans des pays développés dotés d'infrastructures financières et de TIC modernes, atténue sans doute les difficultés rencontrées par les pays en développement lorsqu'il s'agit d'effectuer des réservations en ligne. Les organisations de promotion de ces destinations dans les pays en développement peuvent jouer un rôle déterminant dans le succès des initiatives de tourisme électronique, en aidant l'industrie nationale à participer à la concurrence sur le marché en ligne; elles peuvent même devenir des portails nationaux à l'intention des touristes potentiels. Asiatravelmart.com et Lakbay.Net sont deux exemples d'initiatives de tourisme électronique qui ont su exploiter avec succès ces partenariats dans le monde en développement. 
La société Asiatravelmart.com qui se présente comme le "numéro un de la vente en ligne de voyages en Asie ", exerce une double fonction: elle exploite un système de réservation sur Internet et fait office de chambre de compensation pour les règlements sécurisés du commerce électronique, en collectant les paiements auprès des acheteurs pour le compte des fournisseurs. Dans le monde entier, les particuliers et les entreprises peuvent interagir avec 43000 prestataires et agents de voyages de 200 pays, qui leur proposent 110000 produits sans passer par des intermédiaires. [Source: CNUCED].

La société Kalakbayan Travel Systems a créé Lakbay.Net, un système de réservation nationale de voyages sur Internet, pour fournir aux voyageurs des informations précises sur le tourisme aux Philippines ainsi qu'un dispositif de réservation et de paiement simple d'emploi. Son modèle d'activité en ligne comprend: un site pour le commerce entreprises-particuliers (B2C) ; un portail interentreprises (B2B) pour les professions du tourisme ; des services électroniques à destination des milieux d'affaires, qui se composent d'un système de réservation et de règlement électronique ; un service de commercialisation hors ligne par une chaîne de télévision spécialisée, Lakbay TV. Lakbay est un pionnier du concept d'« appro-tech » qui consiste à utiliser la technologie appropriée à la compétence de chaque membre du réseau, messages SMS, courrier électronique et moyens de communication hors ligne [Source: CNUCED].

159. Les activités touristiques dans les pays en développement peuvent aussi être exercées par des filiales de sociétés internationales comme la société suisse Kuoni Travel, qui possède 300 bureaux dans le monde entier. De même, des entreprises de pays en développement peuvent s'installer à l'étranger pour promouvoir les voyages dans leur pays.

Sita Inbound, filiale de KUONI Inde, est l'un des principaux voyagistes en Inde. Elle existe depuis 45 ans et dispose d'un réseau de bureaux en Inde, avec des correspondants au Népal et au Sri Lanka. En outre, elle a des représentations dans plus de huit autres pays. Sita Inbound organise des voyages en groupe et en individuel dans le sous-continent indien. En 2001, elle a eu une clientèle d'environ 100000 touristes étrangers et réalisé un chiffre d'affaires de 1300 millions d'INR. Ses principaux marchés sont l'Europe, le Royaume-Uni et l'Amérique du nord [Source : Evalueserve].

Travel Corporation of India (TCl) est un voyagiste qui offre des services nationaux et internationaux dans une même structure. Son cœur de métier comprend la gestion de destinations, ainsi que les voyages d'affaires et de loisir. La société possède 27 bureaux en Inde et 10 à l'étranger, situés à des emplacements stratégiques au regard des besoins intégrés de ces voyages. Les implantations étrangères sont en Allemagne, au Royaume-Uni, aux États-Unis, en Espagne, en Chine, au Japon, en Corée du Sud, au Canada et en Australie. TCl est membre d'un certain nombre d'associations internationales de voyagistes telles que IATA [Source : Evalueserve]. 
ANNEXE

Tableau A1. Liste de sociétés multinationales qui sous-traitent à des pays en développement

\begin{tabular}{|c|c|c|c|c|c|}
\hline \multicolumn{6}{|l|}{ Inde } \\
\hline & Sociétés & Opérations & Nature & Vendeur & $\begin{array}{l}\text { Effectifs } \\
\text { (approx) }\end{array}$ \\
\hline 1 & McKinsey & R\&D (logiciels d'application sur mesure) & captive & & 300 \\
\hline 2 & British Airways & $\begin{array}{l}\text { Opérations post-marché pour BA et } 9 \text { autres } \\
\text { compagnies }\end{array}$ & captive & & 1200 \\
\hline 3 & American Express & $\begin{array}{l}\text { Modélisation de fraudes et risques } \\
\text { Exécution des opérations financières } \\
\text { Centre d'appels }\end{array}$ & captive & & \\
\hline 4 & General Electric & $\begin{array}{l}\text { Centre d'appels } \\
\text { Services comptables internationaux } \\
\text { Exécution de transactions }\end{array}$ & captive & & 13000 \\
\hline 5 & Swissair & $\begin{array}{l}\text { Comptabilité et gestion des recettes } \\
\text { Chèques passagers et application des prix } \\
\text { Guichet électronique d'assistance, } \\
\text { renseignements }\end{array}$ & Co-entreprise & & \\
\hline 6 & Netscape Communication & Centre d'appels & captive & & 1200 \\
\hline 7 & Oracle & R\&D & captive & & 2500 \\
\hline 8 & Adobe & R\&D & captive & & \\
\hline 9 & Banque mondiale & $\begin{array}{l}\text { Comptabilité, achats et décaissement des prêts } \\
\text { Comptabilité } \\
\text { Achats et décaissement des prêts }\end{array}$ & captive & & 100 \\
\hline 10 & Accenture & R\&D (logiciel) & captive & & 1000 \\
\hline 11 & Standard Chartered Bank & $\begin{array}{l}\text { Opérations bancaires, Services TI, services de } \\
\text { ressources humaines }\end{array}$ & captive & & 2200 \\
\hline 12 & HSBC & Traitement électronique de données & captive & & 2000 \\
\hline 13 & Banque Mondiale & Services TI et administratifs & Tiers & $\begin{array}{l}\text { Satyam } \\
\text { Computers, HCL } \\
\text { Technology }\end{array}$ & \\
\hline 14 & Bechtel & Bureaux d'études & captive & & 400 \\
\hline 15 & Pfizer & R\&D & captive & & NA \\
\hline
\end{tabular}

\begin{tabular}{|c|c|c|c|c|c|}
\hline \multicolumn{6}{|c|}{ Philippines } \\
\hline & Sociétés & Opérations & Nature & Vendeurs & Effectifs (approx) \\
\hline 1 & $\begin{array}{l}\text { American International } \\
\text { Group (AIG) }\end{array}$ & $\begin{array}{l}\text { Centre d'appels, } \\
\text { Entrée de données et codage }\end{array}$ & Co-entreprise & & \\
\hline 2 & $P \& G$ & $\begin{array}{l}\text { Services comptables/rapports financiers } \\
\text { Services de personnel }\end{array}$ & captive & & \\
\hline 3 & Caltex & Opérations post-marché & captive & & \\
\hline 4 & UPS Asia & Comptabilité financière consolidée & captive & & \\
\hline 5 & SPI technologies & & & & \\
\hline 6 & $\begin{array}{l}\text { Croix rouge internationale } \\
\text { (Suisse) }\end{array}$ & Comptabilité & captive & & \\
\hline 7 & Citibank & $\begin{array}{l}\text { Appui technologique à la société } \\
\text { Services de gestion financière } \\
\text { Aide au développement de systèmes }\end{array}$ & captive & & \\
\hline 8 & Alitalia & $\begin{array}{l}\text { Comptabilité générale et comptabilité } \\
\text { passagers }\end{array}$ & captive & & \\
\hline 9 & $\begin{array}{l}\text { Bechtel Fluor Daniel } \\
\text { Parsons }\end{array}$ & Bureaux d'études & captive & & \\
\hline 10 & Barnes\&Noble AOL & Service Internet & captive & & \\
\hline 11 & Andersen Consulting & $\begin{array}{l}\text { Conception de logiciels/logiciels sur } \\
\text { mesure }\end{array}$ & captive & & \\
\hline
\end{tabular}




\begin{tabular}{|r|l|l|l|l|r|}
\hline \multicolumn{1}{|c|}{ Afrique du Sud } & \multicolumn{1}{|c|}{ Opérations } & Nature & Vendeurs & Effectifs (approx) \\
\hline $\mathbf{1}$ & Swisscard & $\begin{array}{l}\text { Exécution des opérations par carte de } \\
\text { crédit }\end{array}$ & Tiers & Nedcor & \\
\hline $\mathbf{2}$ & Lufthansa & Centre d'appels & captive & & 120 \\
\hline
\end{tabular}

\begin{tabular}{|r|l|l|l|r|r|}
\hline \multicolumn{2}{|c|}{ Chine } & \multicolumn{1}{|c|}{ Opeciétés } & \multicolumn{1}{|c|}{ Nature } & Vendeurs & Effectifs (approx) \\
\hline $\mathbf{1}$ & Microsoft & Centre d'appui technique & captive & & \\
\hline $\mathbf{2}$ & GE Capital & & captive & & 350 \\
\hline
\end{tabular}

\begin{tabular}{|c|c|c|c|c|c|}
\hline Malaisie & & & & & \\
\hline & Sociétés & Opérations & Nature & Vendeurs & Effectifs (approx) \\
\hline 1 & ABN Amro & Services administratifs clientèle & Tiers & Vsource & \\
\hline
\end{tabular}

Source: Evalueserve. 
Tableau A.2. Entreprises de pays en développement figurant dans les 150 principales sociétés de construction classées en fonction du chiffre d'affaires réalisé en dehors du pays d'origine en 2001 (en millions d'USD)

\begin{tabular}{|c|c|c|c|c|c|}
\hline Classement & Entreprises & Pays & $\begin{array}{c}\text { Chiffre d'affaires } \\
\text { international } 2001 \text { (en } \\
\text { millions d'USD) }\end{array}$ & $\begin{array}{c}\text { Chiffre d'affaires } \\
\text { total } 2001 \text { (en millions } \\
\text { d'USD) }\end{array}$ & $\begin{array}{c}\text { Nouveaux } \\
\text { contrats (en } \\
\text { millions } \\
\text { d'USD) }\end{array}$ \\
\hline 12 & $\begin{array}{l}\text { Hyundai } \\
\text { Engineering \& } \\
\text { Construction Co. Ltd }\end{array}$ & Corée & 2036.2 & 4245 & 4709 \\
\hline 22 & $\begin{array}{l}\text { China State Constr. } \\
\text { Engineering Corp. }+\end{array}$ & Chine & 1093.5 & 5815.8 & 7326.9 \\
\hline 29 & $\begin{array}{l}\text { Construtora } \\
\text { Odebrecht }+ \\
\end{array}$ & Brésil & 843 & 1327 & 1890 \\
\hline 41 & $\begin{array}{l}\text { China Harbour } \\
\text { Engineering Co. } \\
\text { (Group) }+\end{array}$ & Chine & 589.1 & 2225.7 & 1953.3 \\
\hline 42 & $\begin{array}{l}\text { Paul Y.-ITC Constr. } \\
\text { Holdings Ltd. }+\end{array}$ & Hong Kong & 586 & 1407 & 833 \\
\hline 43 & SKEC + & Corée & 576 & 1476 & 1568 \\
\hline 46 & Grinaker-LTA Ltd. + & $\begin{array}{l}\text { Afrique du } \\
\text { Sud }\end{array}$ & 492 & 1164 & 1170 \\
\hline 50 & CNMEG + & Chine & 456.6 & 783.1 & 2061.4 \\
\hline 57 & $\begin{array}{l}\text { Shanghai } \\
\text { Construction } \\
\text { (Group) General Co. }\end{array}$ & Chine & 379.5 & 2491.3 & 1814.5 \\
\hline 61 & $\begin{array}{l}\text { Daewoo E\&C Co. } \\
\text { Ltd }+\end{array}$ & Corée & 328 & 2343 & 3093 \\
\hline 68 & $\begin{array}{l}\text { China Civil } \\
\text { Engineering } \\
\text { Construction Corp. }+\end{array}$ & Chine & 277.4 & 286.3 & 252.3 \\
\hline 72 & $\mathrm{CMEC}+$ & Chine & 250.7 & 384.7 & 1582.6 \\
\hline 73 & $\begin{array}{l}\text { China Railway } \\
\text { Engineering Corp. } \\
(\text { CREC })+\end{array}$ & Chine & 249.2 & 4781.8 & 5575.6 \\
\hline 74 & $\begin{array}{l}\text { China Road \& } \\
\text { Bridge Corp. }+\end{array}$ & Chine & 243.4 & 1389.1 & 1895.4 \\
\hline 76 & $\begin{array}{l}\text { Dongfang Electric } \\
\text { Corp. }+\end{array}$ & Chine & 217.2 & 217.2 & 361.4 \\
\hline 78 & $\begin{array}{l}\text { Tekfen Construction } \\
\& \text { Installation Co. } \\
\text { Inc. }+\end{array}$ & Turquie & 196.6 & 337.7 & 318 \\
\hline 83 & $\begin{array}{l}\text { Enka Construction \& } \\
\text { Industry Co. }\end{array}$ & Turquie & 177.6 & 599.2 & 151.3 \\
\hline
\end{tabular}


Tableau A.2 (suite)

\begin{tabular}{|c|c|c|c|c|c|}
\hline $\begin{array}{c}\text { Classe } \\
\text { ment }\end{array}$ & Entreprises & Pays & $\begin{array}{c}\text { Chiffre d'affaires } \\
\text { international } 2001 \\
\text { (en millions d'USD) }\end{array}$ & $\begin{array}{c}\text { Chiffre d'affaires } \\
\text { total } 2001 \text { (en } \\
\text { millions d'USD) }\end{array}$ & $\begin{array}{c}\text { Nouveaux } \\
\text { contrats (en } \\
\text { millions } \\
\text { d'USD) }\end{array}$ \\
\hline 86 & $\begin{array}{l}\text { The Arab Contractors O.A.O \& } \\
\text { Co. }+\end{array}$ & Égypte & 164.4 & 1344.7 & 673.6 \\
\hline 88 & $\begin{array}{l}\text { Solel Boneh International la Ltd } \\
+\end{array}$ & Israël & 157.6 & 157.6 & 344.4 \\
\hline 90 & $\begin{array}{l}\text { China Metallurgical Construction } \\
\text { (Group) Corp. }+\end{array}$ & Chine & 151 & 1737.3 & 2117 \\
\hline 92 & $\begin{array}{l}\text { National Petroleum Construction } \\
\text { Co. (NPCC) }\end{array}$ & $\begin{array}{l}\text { Émirats Arabes } \\
\text { Unis }\end{array}$ & 147 & 296 & 428 \\
\hline 94 & Arabian Construction Co. & Liban & 139.2 & 162.2 & 442.5 \\
\hline 96 & $\begin{array}{l}\text { Construtora Andrade Gutierrez } \\
\text { SA }+\end{array}$ & Brésil & 137 & 544 & 698 \\
\hline 98 & $\begin{array}{lcc}\text { Ssangyong } & \text { Engineering } \quad \& \\
\text { Construction } & \text { Co. Ltd }+\end{array}$ & Corée & 132 & 1100 & 486.5 \\
\hline 99 & $\begin{array}{lll}\text { China } & \text { Petroleum } & \text { Eng. } \\
\text { Construction }+ & \\
\end{array}$ & Chine & 131.9 & 213.9 & 434.2 \\
\hline 100 & $\begin{array}{l}\text { China Jiangsu International } \\
\text { Econ-Tech Coop. Corp. }+\end{array}$ & Chine & 127.6 & 178.3 & 250.2 \\
\hline 102 & $\begin{array}{l}\text { Contracting \& Trading } \quad \text { CAT } \\
\text { Group of Co.s }+\end{array}$ & Liban & 121.6 & 143.7 & 255 \\
\hline 103 & Energoprojekt Group + & Yougoslavie & 121.3 & 165.2 & 188 \\
\hline 105 & Ircon International Ltd & Inde & 115.5 & 179.4 & 91.2 \\
\hline 107 & $\begin{array}{l}\text { China International Water \& } \\
\text { Electric Corp. }+\end{array}$ & Chine & 109.9 & 174.4 & 383.6 \\
\hline 113 & $\begin{array}{lll}\text { China National } & \text { Chemical } \\
\text { Engineering Corp. }+ & \\
\end{array}$ & Chine & 96.5 & 504.2 & 337.7 \\
\hline 115 & $\begin{array}{l}\text { China Railway Construction } \\
\text { Corp. }+\end{array}$ & Chine & 91.5 & 4941.8 & 4901.6 \\
\hline 116 & $\begin{array}{lcc}\text { China } & \text { National } & \text { Overseas } \\
\text { Engineering Corp. }+ & \\
\end{array}$ & Chine & 89.8 & 93.3 & 115.3 \\
\hline 118 & $\begin{array}{l}\text { TEKSER Construction Industry } \\
\text { and Trading Inc. + }\end{array}$ & Turquie & 86.1 & 99.5 & ND \\
\hline 119 & $\begin{array}{l}\text { China Nat'l Complete Plant } \\
\text { Import \& Export Corp. }+\end{array}$ & Chine & 85.4 & 85.4 & 83.6 \\
\hline 120 & $\begin{array}{lll}\text { China } & \text { Wanbao } & \text { Engineering } \\
\text { Corp. } & & \\
\end{array}$ & Chine & 83.5 & 99.9 & 110.3 \\
\hline 121 & China Wu Yi Corp. + & Chine & 80.8 & 131.2 & 112.9 \\
\hline 123 & STFA Construction Group & Turquie & 76.2 & 133.9 & 86.3 \\
\hline
\end{tabular}


TD/TC/WP(2003)23/FINAL

Tableau A.2 (suite)

\begin{tabular}{|c|c|c|c|c|c|}
\hline Classement & Entreprises & Pays & $\begin{array}{c}\text { Chiffre d'affaires } \\
\text { international } 2001 \\
\text { (en millions d'USD) }\end{array}$ & $\begin{array}{c}\text { Chiffre d'affaires } \\
\text { total } 2001 \text { (en millions } \\
\text { d'USD) }\end{array}$ & $\begin{array}{c}\text { Nouveaux } \\
\text { contrats (en } \\
\text { millions } \\
\text { d'USD) }\end{array}$ \\
\hline 125 & Bayindir Construction Inc. & Turquie & 73.8 & 179.1 & ND \\
\hline 127 & $\begin{array}{l}\text { Hanjin Heavy Industry \& } \\
\text { Construction }\end{array}$ & Corée & 72.8 & 675.8 & 991.2 \\
\hline 128 & $\begin{array}{l}\text { China Nat'l Water Res. \& } \\
\text { Hydro Engineering Corp. } \\
+\end{array}$ & Chine & 71.4 & 1555.1 & 1945 \\
\hline 129 & GAMA AS + & Turquie & 70.8 & 244.3 & 278.8 \\
\hline 132 & $\begin{array}{l}\text { RSEA Engineering Corp. } \\
+\end{array}$ & Taïwan & 64 & 752.4 & 967.7 \\
\hline 133 & China Shanghai SFECO + & Chine & 63 & 63 & 156.9 \\
\hline 138 & Glavbolgarstroy & Bulgarie & 57 & 98.3 & 52 \\
\hline 139 & $\begin{array}{l}\text { Harbin } \\
\text { Engineering Co. Ltd. }\end{array}$ & Chine & 55.5 & 96.4 & 286.4 \\
\hline 141 & $\begin{array}{ll}\text { Zhejiang } & \text { Construction } \\
\text { Engineering } & \text { Group Co. } \\
\text { Ltd. }+ & \end{array}$ & Chine & 51 & 953.6 & 907.8 \\
\hline 144 & CTCI Corp. + & Taïwan & 48.4 & 305.2 & 542.2 \\
\hline 146 & $\begin{array}{l}\text { Granit Construction Stock } \\
\text { Co. }\end{array}$ & $\begin{array}{l}\text { République de } \\
\text { Macédoine }\end{array}$ & 45 & 181 & 127 \\
\hline 147 & 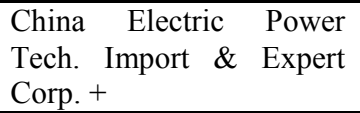 & Chine & 44.2 & 302 & 333.1 \\
\hline 149 & $\begin{array}{l}\text { Italian-Thai Development } \\
\text { PCL }+\end{array}$ & Thaïlande & 43.5 & 401 & 548.9 \\
\hline
\end{tabular}

Source : 225 plus grandes sociétés ENR (Engeneering News Record) (C) The McGraw-Hill Companies, Inc (http://enr.construction.com). Critères : les sociétés sont classées en fonction du chiffre d'affaires en millions d'USD des activités de construction réalisées en dehors de leur pays d'origine en 2001. Celles dont les filiales sont incluses (+) sont classées en fonction du rang de la société. ND =Non disponible. Les chiffres cités englobent les contrats d'entreprise générale, les participations à des co-entreprises, la sous-traitance, les contrats conception-construction et les contrats de gestion de construction « à risque », pour lesquels une firme assume des risques similaires à ceux d'une entreprise générale. Ils prennent aussi en compte la valeur des équipements installés, quand une entreprise a la responsabilité principale pour les spécifier et les fournir dans le cadre d'un contrat de construction. Note: la société classée $27^{\text {ème }}$ au niveau mondial, Joannou \& Paraskevaides (activités étrangères) est officiellement rattachée à Guernesey (Royaume-Uni), mais elle a été créée à Chypre. 
Tableau A.3. Les vingt principaux terminaux de conteneurs et leur débit en 1999

\begin{tabular}{lll}
\hline Classement & Ports & EVP* \\
& & \\
\hline 1 & Hong Kong Chine & 16100 \\
2 & Singapour & 15900 \\
3 & Kaohsiung (Taipeh chinois) & 6985 \\
4 & Busan (Corée) & 6439 \\
5 & Rotterdam & 6400 \\
6 & Long Beach (États-Unis) & 4408 \\
7 & Shanghai & 4210 \\
8 & Los Angeles & 3829 \\
9 & Hambourg & 3750 \\
10 & Anvers & 3614 \\
11 & New York/New Jersey & 2863 \\
12 & Dubaï & 2845 \\
13 & Felixstowe (Royaume-Uni) & 2700 \\
13 & Tokyo & 2700 \\
14 & Port Klang (Malaisie) & 2550 \\
15 & Tanjung Priok (Indonésie) & 2273 \\
16 & Gioia Tauro (Italie) & 2253 \\
17 & Kobe & 2200 \\
17 & Yokohama & 2200 \\
18 & Brême & 2181 \\
19 & Manille & 2104 \\
20 & San Juan & 2085 \\
\hline Total des 20 & & 109221 \\
& & \\
\hline
\end{tabular}

* = équivalent vingt pieds - c'est-à-dire conteneurs. Source : Mukherjee, (2001) 
Tableau A.4. Entreprises de pays en développement figurant dans les 150 premières sociétés mondiales de conception de projet (en architecture et ingénierie), classées en fonction du chiffre d'affaires réalisé pour des projets à l'étranger en 2001

\begin{tabular}{|c|c|c|c|c|c|}
\hline Classement & Entreprises & Pays & Types d'entreprise & $\begin{array}{l}\text { Chiffre d'affaires } \\
\text { international en } \\
2001 \text { (en millions } \\
\text { d'USD) }\end{array}$ & $\begin{array}{c}\text { Chiffre } \\
\text { d'affaires } \\
\text { international } \\
\text { en \% du C.A. } \\
\text { total }\end{array}$ \\
\hline 15 & $\begin{array}{l}\text { Dar Al-Handasah } \\
\text { Consultants }\end{array}$ & Égypte & EA & 350 & 99 \\
\hline 64 & Sinopec Engineering Inc. & Chine & $\mathrm{EC}$ & 50 & 21 \\
\hline 71 & Khatib \& Alami & Liban & EA & 39.9 & 85 \\
\hline 73 & $\begin{array}{l}\text { TAHAL Consulting } \\
\text { Engineers }\end{array}$ & Israël & $\mathrm{E}$ & 39.7 & 73 \\
\hline 92 & SKEC & Corée & $\mathrm{EC}$ & 24.2 & 11 \\
\hline 102 & $\begin{array}{l}\text { China International Water } \\
\text { and Electric Corp. }\end{array}$ & Chine & $\mathrm{EC}$ & 20.4 & 75 \\
\hline 112 & CNMEG & Chine & $\mathrm{EC}$ & 16.1 & 18 \\
\hline 113 & $\begin{array}{l}\text { China National Chemical } \\
\text { Engineering Corporation }\end{array}$ & Chine & EAC & 15.9 & 30 \\
\hline 122 & Africon & Afrique du Sud & $\mathrm{E}$ & 12.9 & 34 \\
\hline 132 & $\begin{array}{l}\text { Beijing Urban Construction } \\
\text { Group }\end{array}$ & Chine & $\mathrm{AEC}$ & 10.7 & 30 \\
\hline 133 & CTCI Corp. & Taïwan & $\mathrm{EC}$ & 10.7 & 23 \\
\hline 139 & $\begin{array}{l}\text { China Huangqiu Chemical } \\
\text { Engineering Corp. }\end{array}$ & Chine & $\mathrm{EC}$ & 9.7 & 67 \\
\hline 142 & $\begin{array}{l}\text { Chengdu Chemical } \\
\text { Engineering }\end{array}$ & Chine & $\overline{\mathrm{EC}}$ & 9.3 & 42 \\
\hline 147 & $\begin{array}{l}\text { China Power Engineering } \\
\text { Consulting }\end{array}$ & Chine & $\mathrm{EC}$ & 8.3 & 7 \\
\hline
\end{tabular}

Source : 225 plus grandes sociétés ENR (C) The McGraw-Hill Companies, Inc (http://enr.construction.com). A=architecture ; $\mathrm{E}=$ conception ; $\mathrm{EC}=$ conception-constructeur ; $\mathrm{AE}=$ architecture-conception ; $\mathrm{EA}=$ conception-architecture Les entreprises se sont classées elles-mêmes. 


\section{PARTIE II}

\section{MODÉLISATION DES AVANTAGES ÉCONOMIQUES DE LA LIBÉRALISATION DES ÉCHANGES DE SERVICES}

\section{INTRODUCTION}

160. Cette partie examine les conséquences, pour les pays développés comme pour les pays en développement, d'une libéralisation des échanges internationaux dans les secteurs de services. Un grand nombre de pays en développement ayant le sentiment qu'ils seront globalement pénalisés par cette évolution, la présente étude propose une synthèse et une analyse des documents existants sur l'évaluation quantitative des effets d'une telle libéralisation.

\section{Pour une libéralisation multilatérale}

161. Dans la plupart des économies, les services sont en passe de devenir le premier secteur d'activité, en volume comme en importance. Non seulement ce secteur génère la plus grosse partie des emplois et des recettes, mais de nombreux services - finances, télécommunications et transport - fournissent un apport intermédiaire essentiel pour la production d'autres biens et services. L'efficacité de ce secteur est indispensable à celle de l'économie générale. Par ailleurs, tous les pays en développement enregistrent une augmentation considérable du volume global des échanges de services, même si, dans la plupart des cas, ces échanges étaient au départ très limités.

162. Les restrictions aux échanges de services entraînent des coûts, généralement sous la forme de prix plus élevés pour les entreprises et les consommateurs. Elles limitent la concurrence intérieure et internationale, réduisent l'efficience et permettent aux fournisseurs en place de majorer leurs prix par rapport à ceux d'un marché concurrentiel. En évaluant la mesure dans laquelle les restrictions augmentent les prix ou entravent la concurrence, on fait ressortir les avantages qu'entraînerait leur suppression pour les consommateurs, les décideurs et les négociateurs commerciaux. La libéralisation multilatérale peut être considérée dans ce contexte comme un élément inestimable des efforts généraux déployés par les pays pour profiter des avantages de la concurrence. En fait, les modèles étudiés ici, en dépit de leurs différences techniques, indiquent tous que la libéralisation des services apporte des avantages avant tout, non pas aux partenaires commerciaux des pays où se produit cette libéralisation, mais aux économies libéralisées ellesmêmes.

\section{Sujets de préoccupation dans les pays en développement : quelques exemples}

163. Le secteur des services étant inefficace et non concurrentiel dans un grand nombre de pays en développement, ceux-ci ont le sentiment qu'une libéralisation mondiale des échanges dans ce domaine leur sera finalement préjudiciable. Ce point de vue va à l'encontre des arguments des économistes : selon eux, en effet, la baisse des prix qui découlant de la libéralisation jouerait en faveur des consommateurs; quant aux pays exportateurs et importateurs, ils bénéficieraient tout à la fois de la mise en balance des avantages 
comparatifs, de meilleures possibilités d'accès aux marchés étrangers et de l'efficacité accrue des marchés intérieurs. Les craintes exprimées par les pays en développement contredisent également l'opinion, très répandue, selon laquelle de nombreux services nécessitent une main-d'œuvre importante, ce qui — de l'avis des spécialistes - devrait constituer un avantage pour les plus pauvres des pays en développement en ce qui concerne la prestation de services.

164. Cette réticence des pays en développement à l'égard d'une libéralisation du commerce des services à l'échelle mondiale semble être le reflet de deux grandes inquiétudes. Premièrement, ces pays considèrent généralement que toute libéralisation négociée de ces échanges donnera des résultats en grande partie déséquilibrés. Deuxièmement, ils s'interrogent quant à la nature et à l'ampleur des ajustements que cette libéralisation pourrait nécessiter dans leurs propres économies.

165. La conviction qu'une telle négociation serait d'office partiale repose sur l'hypothèse selon laquelle, en cas de négociation sur la libéralisation des échanges de services à l'échelle multilatérale (voire régionale) dans le cadre de l'OMC, les fournisseurs de services des pays développés obtiendront probablement un bien meilleur accès aux marchés de services des pays en développement, alors que l'inverse (c'est-à-dire un meilleur accès des fournisseurs de services des pays en développement aux marchés des pays développés) aura peu de chances de se produire. L'une des raisons invoquées à l'appui de ce point de vue tient au décalage entre les capacités de négociation des différents pays; une autre des raisons mentionnées est l'ampleur des engagements pris à l'issue du Cycle d'Uruguay.

166. La deuxième préoccupation exprimée par les pays en développement concerne la nature et l'ampleur des ajustements qu'une libéralisation des échanges de services pourrait nécessiter dans leurs économies respectives. L'un des aspects mentionnés porte sur l'éventualité d'une participation et d'un contrôle étrangers majoritaires dans la prestation de services pour certains secteurs clés, ce qui suscite des inquiétudes liées à la sécurité mais aussi aux aspects culturels. Par exemple, certains pays jugent inacceptable que des entités étrangères puissent avoir accès aux écritures bancaires ou aux données financières concernant leurs résidents, voire même exercer un contrôle à cet égard. En outre, une industrie cinématographique ou télévisuelle très vivante peut être considérée comme faisant partie intégrante de l'identité culturelle du pays concerné. Outre ces motifs d'inquiétude, il faut citer l'ampleur des adaptations qui pourraient s'avérer nécessaires sur le marché du travail si des banques nationales venaient à être remplacées par des banques étrangères (ou des compagnies aériennes intérieures par des compagnies étrangères), ainsi que les autres modifications importantes que la libéralisation pourrait entraîner dans l'organisation des secteurs à forte densité de main-d'œuvre.

167. Plusieurs points peuvent être soulignés, dans un premier temps, pour répondre à ces différents sujets de préoccupation. Tout d'abord, les mécanismes qui permettent un meilleur accès aux marchés des fournisseurs de services des pays en développement sont en train d'évoluer, en raison de la réforme en cours dans le cadre de l'OCDE en matière de réglementation; il est vrai, cependant, que ces modifications ne sont pas nécessairement prises en compte dans les engagements prévus à l'OMC au titre de l'Accord général sur le commerce des services (AGCS). Les négociations en cours offrent la possibilité de refléter cette ouverture dans les engagements concernant les secteurs et les modes de fourniture qui présentent un intérêt pour les pays en développement. De plus, les échanges entre pays en développement constituent un volet important, mais négligé, de l'accès aux marchés; il convient enfin de souligner les possibilités qu'offrira, pour certains pays en développement, la libéralisation des marchés intervenant dans d'autres.

168. Par ailleurs, l'évolution qui découlera de la libéralisation, tout en représentant un défi, pourra être facilitée par l'adoption de structures politiques appropriées; les faits montrent à ce propos que les coûts d'adaptation pourraient être moins élevés pour les échanges de services que dans le cas du commerce de produits manufacturés. Dans ce contexte, il sera essentiel de prendre en considération la nature, le rythme et le déroulement du processus de libéralisation afin d'effectuer au mieux les ajustements nécessaires en 
matière de gestion et de faire en sorte que ce processus s'appuie sur des cadres réglementaires solides. La structure de l'AGCS, par sa souplesse intrinsèque et l'importance qu'elle accorde au caractère progressif de la libéralisation, traduit bien la nécessité d'un examen attentif de ces différents aspects. Pour de nombreux pays en développement, cependant, la mise en place de réglementations appropriées pour régir ce processus - ainsi que la possibilité d'en assurer l'application - nécessitera un renforcement considérable des capacités.

169. La présente étude se propose de contribuer à la réflexion menée par les membres de l'OMC sur ces différentes questions en présentant une évaluation quantitative des effets de la libéralisation du commerce des services, pour les pays en développement comme pour les pays développés. 


\section{PRINCIPALES CONCLUSIONS DES ÉTUDES DISPONIBLES}

\section{Méthodologie}

170. Cette partie de l'étude vise à fournir des évaluations objectives et quantifiables des avantages d'une libéralisation des échanges de services, à partir des calculs économétriques et des travaux de modélisation les plus récents effectués sur le sujet, ainsi que des études menées précédemment par la Direction des échanges ${ }^{13}$. Qu'il s'agisse d'études récentes ou des travaux mentionnés dans des documents plus anciens de l'OCDE, les résultats qui concernent les pays en développement et les pays développés sont considérés séparément afin de permettre une analyse comparative des conclusions. A propos des obstacles aux échanges de services, l'étude en présente une estimation pour les pays en développement, analysant les variations en fonction des secteurs, et compare ces données avec les estimations relatives aux économies avancées. De même, pour ce qui est de la modélisation des conséquences que la suppression de ces obstacles auraient sur le bien-être, l'étude présente un tableau comparatif des avantages que pourraient en retirer les pays en développement et les pays développés, pour le secteur des services dans son ensemble et pour différentes catégories de services considérées séparément.

\section{Obstacles aux échanges de services}

171. L'évaluation quantitative des effets sur le bien-être d'une libéralisation du commerce des services nécessite deux étapes : une estimation des obstacles aux échanges et la prise en compte de cette estimation dans un cadre d'équilibre général (Jomini et al., 2002). Il est donc essentiel de mesurer l'ampleur des restrictions et des obstacles pour déterminer correctement l'impact d'une libéralisation. Cette étape est également importante en soi, car «elle donne une vue d'ensemble des coûts induits par le choix d'une attitude protectionniste de la part des gouvernements ainsi que des bénéfices qui découleraient d'une levée de ces obstacles, stimulant ainsi la réforme » (McGuire, 2002).

172. Les documents évaluant la nature et l'ampleur des obstacles au commerce des services appliquent généralement les méthodes élaborées antérieurement pour mesurer l'importance des barrières non tarifaires appliquées aux produits manufacturés. ${ }^{14}$ Dès lors, les outils utilisés pour évaluer ces obstacles et les

Voir les études antérieures sur l'évaluation des obstacles aux échanges de services et les avantages d'une libéralisation en termes de bien-être : «Quantification des conséquences pour le bien-être national des obstacles au commerce de services : tour d'horizon de différents ouvrages » [TD/TC/WP(2000)24/FINAL].

Les obstacles non tarifaires découlent généralement de mesures prises par les gouvernements. Celles-ci peuvent concerner l'entrée sur le marché et les activités de fournisseurs étrangers, mais aussi de nouveaux fournisseurs nationaux, et provoquer ainsi directement la hausse des prix ou des coûts des produits extérieurs et intérieurs. Les barrières non tarifaires conventionnelles peuvent être considérées comme des instruments d'accès aux marchés ou des mesures relatives au traitement national, et peuvent prendre la forme de restrictions quantitatives, de limitations fondées sur les prix, d'exigences en matière de licences ou d'homologation, et de discrimination pour l'accès aux systèmes de distribution ou de communication. De même, en ce qui concerne les services, les restrictions aux échanges peuvent limiter l'accès aux marchés ou instaurer une discrimination à l'encontre de fournisseurs étrangers ; par ailleurs, les obstacles concernent soit le droit d'établissement (mode 3), soit le droit de fournir ou de consommer des services 
conséquences de la libéralisation restent quelque peu limités : il faudra les améliorer afin de tenir compte des caractéristiques propres au secteur des services.

173. Outre le plus large éventail d'obstacles à prendre en compte que dans le cas des marchandises, il importe de déterminer si les réglementations en vigueur constituent réellement un frein aux échanges : on ne peut en effet se contenter d'un amalgame entre règlements et obstacles. Par ailleurs, les règles concernant les services étant généralement conçues à l'appui d'un certain nombre d'objectifs politiques, il pourrait s'avérer utile d'établir si elles ne sont pas plus pesantes que nécessaires dans la poursuite de ces objectifs et si d'autres mesures, tout aussi efficaces mais moins restrictives, ne seraient pas envisageables. Ces mesures de portée générale étant difficiles à quantifier, il faudrait en affiner les modalités.

174. Ces dernières années ont vu la mise au point de plusieurs méthodes nouvelles afin de mesurer l'ampleur des obstacles au commerce des services [voir l'Appendice 1 pour un examen plus approfondi de la question]. En particulier, de nouvelles études ont été menées afin de tenter, pour la première fois, de prendre en compte l'impact de la réglementation, en proposant un traitement sectoriel et modal de la question, ainsi que des méthodes de pondération plus modernes. Tout en proposant des méthodes novatrices, elles se fondent sur les données disponibles, qui s'arrêtent généralement au milieu ou à la fin des années 90, de sorte que l'évolution récente du marché des services dans certains pays n'est pas toujours bien prise en compte. En outre, ces études restent limitées dans la mesure où elles se servent des listes d'engagements au titre de l'AGCS comme sources d'information sur les obstacles à la libéralisation des échanges de services. En effet, le niveau réel de libéralisation d'un secteur est souvent plus élevé que celui des listes AGCS ; de plus, certains types de mesures n'ont pas besoin d'être inscrites sur ces listes.

175. Pour récapituler les différentes méthodes utilisées pour l'évaluation des obstacles, la présente étude suit la classification proposée par Deardoff et Stern (1998) et distingue trois types de mesures, reposant respectivement sur la fréquence, sur la quantité et sur l'évolution des prix. Toutefois, ces méthodes doivent être adaptées pour tenir compte des spécificités du commerce de services. Dans ce contexte, il est également possible de distinguer entre les méthodes utilisées pour mesurer le niveau des restrictions appliquées aux services et celles qui permettent de mesurer les effets de ces restrictions. De manière générale, on trouve dans la première catégorie les mesures portant sur la fréquence, tandis que la seconde regroupe les mesures privilégiant les notions de quantité et de prix.

\section{Mesures tenant compte essentiellement de la fréquence des obstacles}

176. Les restrictions au commerce de services sont généralement mesurées au moyen d'un indice, c'est-à-dire par l'attribution de notes et de coefficients de pondération, un système qui permet de convertir des informations qualitatives en mesures quantitatives, en fonction du nombre et de la rigueur des restrictions. Depuis les toutes premières estimations chiffrées de Hoekman (1995) fondées sur les listes d'engagements relatifs à l'AGCS, la qualité et la complexité des indices proposés ont augmenté progressivement. Les améliorations les plus sensibles concernent l'éventail des obstacles considérés — les sources d'information étant de plus en plus nombreuses - ainsi que les techniques d'évaluation et les méthodes de pondération employées.

177. Hardin et Holmes (1997) ont mis au point des indices de fréquence afin de mesurer l'ampleur des obstacles à l'investissement direct étranger (IDE) dans un certain nombre de secteurs d'activité. Ces indices présentent trois caractéristiques majeures : premièrement, les auteurs ont choisi de ne pas se limiter aux listes AGCS et ont ainsi calculé l'importance des restrictions au commerce à partir d'informations

dans un pays étranger (mode 1, 2, 3, 4). (Voir Findlay et Warren (2000), Chen et Schembri (2002) et McGuire (2002b) pour la présentation détaillée de documents consacrés à ces questions et des méthodes utilisées pour évaluer les obstacles au commerce des services). 
puisées dans les Plans d'action individuels (PAI) des pays de l'APEC, qui offrent l'avantage de fournir un tableau plus précis des obstacles réels. Deuxièmement, ces indices couvrent une large gamme de restrictions. Enfin, des facteurs de pondération sont attribués aux différents types de barrières en fonction de l'impact économique supposé des différentes politiques constatées. Les résultats montrent que les les restrictions les plus fortes à l'IDE concernent les télécommunications et le secteur financier, alors que les services aux entreprises, la distribution, l'environnement et les loisirs sont les secteurs où les limitations sont les plus faibles. Hardin et Holmes ont appliqué leur méthode à 15 pays de l'APEC, ce qui leur a permis de constater que la République de Corée, l'Indonésie, la Thaïlande et la Chine figurent parmi les économies les plus protectrices ${ }^{15}$.

178. Dans le cadre d'un projet commun, l'Australian Productivity Commission et l'Australian National University ont conçu une méthode de mesure de la fréquence des obstacles pour six secteurs de services : télécommunications (Warren, 2001), banque (McGuire et Schele, 2001), transport maritime (McGuire et al., 2001), éducation (Kemp, 2001), distribution (Kalirajan, 2000) et services professionnels (Nguyen Hong 2000). Le taux de restriction des échanges a été établi d'après des données provenant de sources différentes, dont plusieurs organisations internationales et organismes de commerce nationaux. Comme dans le cas de Hardin et Holmes, ces travaux conjoints marquent un progrès par rapport à la méthode de Hoekman, dont la principale limite était de s'en tenir exclusivement aux listes AGCS, lesquelles ne donnent qu'un aperçu très incomplet des barrières aux échanges de services. On relèvera toutefois que la richesse des informations recueillies et la complexité de la méthode élaborée par l'Australian Productivity Commission et l'Australian National University varient d'un secteur à l'autre.

179. D'autres méthodes ont été mises au point pour des secteurs particuliers, notamment par Marko (1998) concernant le secteur des télécommunications, Claessens et Glaessner (1998) et Mattoo (1998) pour les services financiers, et Colecchia (2001) pour les services professionnels.

180. Toutes les études recensées parviennent à la conclusion que, quels que soient le secteur concerné et la méthodologie retenue, les restrictions sont en moyenne plus importantes dans les pays en développement que dans les pays développés. Les paragraphes qui suivent résument brièvement les principales constatations quant aux obstacles existants dans les pays en développement pour six secteurs particuliers. En complément de cette analyse, on trouvera à l'Appendice 5 des tableaux illustrant la situation relative à certains secteurs. Tout en donnant les chiffres relatifs à certains pays, ces tableaux fournissent des notes moyennes globales pour les économies à revenu faible ou moyen et les économies à revenu élevé.

\section{Services financiers et bancaires}

181. Claessens et Glaessner (1998), Mattoo (1998), McGuire (1998), McGuire et Schuele (1999 et 2000) ont tous élaboré des méthodes afin de mesurer l'ampleur des obstacles aux échanges de services financiers. Bien qu'elles mettent en œuvre des méthodologies différentes, toutes ces études relèvent que les économies d'Asie et d'Amérique du Sud sont beaucoup plus restrictives et discriminatoires que celles des pays d'Amérique du Nord et d'Europe. A partir de cette constatation, McGuire (2002) a établi un tableau comparatif reprenant les résultats des études susmentionnées, auxquels il a ajouté des notes en fonction de la sévérité des obstacles (de 0 à 1 , c'est-à-dire du régime le moins restrictif au régime le plus restrictif. [Voir Appendice 4]

15 Les constatations de Hardin et Holmes se fondent sur des données du milieu des années 90. L’évolution récente de la libéralisation du marché des services dans les pays de l'APEC n'est pas prise en compte dans ce document. 
182. Kalirajan (2001) a calculé un taux de restriction des échanges de services de distribution pour 38 économies englobant la région Asie-Pacifique, l'Europe et l'Amérique. Il constate à propos de ce secteur que, dans l'ensemble, les économies à revenu faible ou moyen sont davantage protégées que les économies à revenu élevé. Les marchés les plus fermés sont ceux de l'Inde, de l'Indonésie, de la République de Corée, de la Malaisie, des Philippines et de la Thaillande. Parmi les mesures restrictives les plus répandues dans ces pays figurent les interdictions frappant le commerce de détail, la limitation du nombre de licences d'importation accordées aux étrangers, ainsi que les limitations et les exigences de performances imposées à la participation de capitaux propres étrangers dans des entreprises nationales. Cependant, la libéralisation récente du marché de la distribution dans certains des pays étudiés n'est pas prise en compte. La Corée, par exemple, a procédé à une importante libéralisation du secteur de la distribution dans la deuxième moitié des années 90 .

\section{Services maritimes}

183. McGuire et al (2000) ont évalué l'ampleur et la nature des restrictions relatives aux services maritimes pour 35 pays répartis dans le monde entier. En moyenne, les restrictions entravant le commerce de services maritimes sont plus importantes dans les économies à revenu faible ou moyen que dans les économies à revenu élevé. Toutefois, les chiffres varient considérablement d'un pays à un autre. Si le Canada, les États membres de l'UE, la Nouvelle-Zélande et Singapour sont des marchés relativement ouverts, l'Inde, l'Indonésie, la République de Corée, les Philippines, la Thaïlande, la Malaisie, les États-Unis, le Brésil et le Chili sont les pays qui imposent les barrières les plus strictes aux étrangers. Par ailleurs, c'est en Turquie, aux Philippines, en Thaillande, au Chili et aux États-Unis que les obstacles sont les plus discriminatoires.

\section{Services professionnels}

184. Nguyen-Hong (2000) a calculé le taux de restriction des échanges dans 34 économies de la région Asie-Pacifique, d'Europe et d'Amérique. Il ressort de ses travaux que les pays où les obstacles aux échanges de services professionnels sont les plus élevés sont l'Indonésie, la Malaisie, le Mexique, les Philippines et la Turquie. Tous ces pays imposent des conditions de nationalité et de résidence pour autoriser la prestation de tels services. Dans certains secteurs professionnels, ils exigent des sociétés étrangères désireuses d'accéder aux marchés qu'elles s'associent à des entreprises locales, testent les professionnels admis à exercer leur activité en fonction des besoins économiques et limitent les formes d'établissement ainsi que les investissements étrangers dans les firmes locales. Ce sont l'Indonésie, la Malaisie et les Philippines qui pratiquent les mesures les plus discriminatoires à l'encontre des étrangers. L'Argentine et l'Afrique du Sud sont, en revanche, les moins restrictifs des pays en développement : reconnaissant les qualifications des prestataires étrangers, elles se montrent également plus souples en ce qui concerne la pénétration de sociétés et d'intérêts étrangers.

\section{Télécommunications}

185. Selon Warren (2001), le Burkina Faso, le Costa Rica, l'Éthiopie, Malte, la Syrie et la Tunisie sont, parmi 136 pays répartis dans le monde entier, ceux qui appliquent la réglementation la plus restrictive dans le secteur des télécommunications. Pour la région Asie-Pacifique, l'Europe et l'Amérique, les obstacles les plus importants sont imposés par l'Inde, l'Indonésie, la République de Corée, la Thaïlande et la Turquie. Ces économies se caractérisent par des limitations très strictes quant à l'investissement direct étranger (IDE) dans la téléphonie fixe et mobile, les restrictions étant d'ailleurs plus marquées pour la première que pour la seconde. Par ailleurs, l'importance des obstacles varie en ce qui 
concerne l'accès à des lignes et réseaux en location : l'Argentine, le Brésil et le Chili sont, à cet égard, les pays les moins restrictifs ${ }^{16}$.

\section{Éducation}

186. Kemp (2001) a étudié les obstacles aux échanges de services dans le domaine de l'éducation. A l'instar d'Hoekman (1995), il utilise comme source de données les listes AGCS afin de cerner la nature des restrictions au commerce et à l'investissement, et d'en mesurer l'ampleur. Sa démarche est cependant novatrice en ce sens que le calcul des taux tient compte de la moyenne pondérée des notes attribuées pour cinq sous-secteurs de l'éducation, des quatre modes de fourniture de services et de deux catégories de limitations (accès au marché et traitement national). Deux taux sont proposés, qui se différencient par la méthode d'attribution de la note : tandis que, dans la première méthode, les secteurs ne figurant pas dans les listes AGCS sont considérés comme soumis à un régime très restrictif (ce qui leur vaut la note 1), la seconde estime au contraire qu'aucune restriction ne frappe ces secteurs et leur attribue la note 0 . Les taux de restrictivité obtenus sont donc très différents. Dans le premier cas, le secteur de l'éducation apparaît comme assez protégé, la plupart des pays obtenant une note supérieure à 0.5 , et un seul (le Lesotho) moins de 0.3. Dans le second cas, le secteur apparaît au contraire relativement ouvert : seul le Japon obtient une note supérieure à 0.5 , et 21 pays restent en deçà de 0.3 . Ni l'un ni l'autre de ces deux indices ne font ressortir de différence notable entre le groupe des pays en développement et celui des pays développés.

187. La différence constatée dans les résultats obtenus par Kemp souligne la difficulté d'une évaluation des barrières existantes à partir des engagements pris dans le cadre de l'AGCS ; par conséquent, les deux séries de taux de restriction doivent être considérées comme les deux extrémités d'une fourchette. Un marché peut être ouvert même en l'absence d'engagements; en revanche, il se peut que les engagements pris ne soient pas représentatifs du degré d'ouverture d'un marché, en raison, par exemple, d'une libéralisation entreprise unilatéralement depuis le Cycle d'Uruguay. Si les faits donnent à penser que les échanges internationaux se développent pour certains services éducatifs, les engagements souscrits par les pays au titre de l'AGCS ne reflètent pas toujours cette évolution. Un premier examen des engagements de membres de l'OMC connus pour pratiquer le commerce de services dans le secteur de l'enseignement post-secondaire semble indiquer qu'il est probablement inexact de voir dans l'absence d'engagement le signe d'un marché fermé ; par contre, l'hypothèse inverse (consistant à déduire de l'absence d'engagements qu'un marché est totalement ouvert) reviendrait probablement à surestimer le degré d'ouverture réel du marché concerné.

\section{Mesures quantitatives de la restrictivité des obstacles au commerce}

188. Ces mesures découlent généralement des modèles standard d'évaluation des échanges, c'est-àdire le modèle de Hecksher Olhin sur les avantages comparatifs ou celui de Helpman-Krugman pour la différenciation des produits, et donnent lieu à des estimations économétriques par le biais d'équations gravitationnelles. Le raisonnement qui sous-tend cette approche est très similaire à celui qui détermine les méthodes d'évaluation des échanges de marchandises. Il s'agit de déterminer ce que ces échanges seraient en l'absence de barrières et de comparer ces estimations à la situation réelle. Les obstacles sont évalués en termes de résiduels (différence entre les volumes réel et possible des échanges) ou au moyen de variables

16

Cependant, les travaux de Warren ne rendent pas compte de l'évolution récente de la libéralisation des services dans les pays mentionnés. Après la crise financière asiatique de 1997, la Corée a ainsi beaucoup progressé dans la libéralisation de différents secteurs. D'importantes restrictions ont été supprimées dans les services de télécommunications. En avril 2001, le gouvernement a autorisé les entreprises étrangères à prendre des prises de participation pouvant atteindre $49 \%$ dans les services de télécommunications filaires et sans fil. 
indicatrices ${ }^{17}$. Jusqu'à ces derniers temps, cependant, il était difficile d'appliquer ce cadre à l'étude des obstacles en raison du caractère insuffisant des données bilatérales (de pays à pays) concernant les échanges de services ${ }^{18}$.

189. En dépit du manque de données, trois documents présentent des méthodes de mesure des obstacles fondées sur la quantité : Hoekman et Francois (1999), Fink, Mattoo et Neagu (2001) et Warren (2001). Les deux premiers définissent un modèle gravitationnel visant à expliquer les échanges entre les États-Unis et leurs principaux partenaires commerciaux, alors que le dernier présente un système d'évaluation des restrictions aux échanges de services dans le secteur des télécommunications. Warren calcule l'ampleur des entraves à partir d'une enquête sur les politiques en vigueur plutôt qu'en extrapolant d'après les engagements pris dans le cadre de négociations commerciales. Il s'appuie sur les quantités consommées plutôt que sur le volume des échanges. Son document ne comporte aucune estimation de résiduels ou de variables. Au lieu de cela, un taux de restriction des échanges permet d'estimer l'impact des entraves au commerce de services. Warren calcule cinq taux différents pour 136 pays développés ou en développement. Il ressort de son analyse que neuf pays développés et un pays en développement (le Chili) figurent parmi les économies les plus libérales, les dix pays les plus fermés étant tous des pays en développement : six en Afrique (Burkina Faso, Ethiopie, Tunisie, Angola, Mali, Mozambique), 1 en Amérique centrale (Costa Rica) et 3 au Proche-Orient ou au Sud de la Méditerranée (Syrie, Jordanie, et Malte).

190. La préférence donnée par Warren aux quantités consommées plutôt qu'au volume des échanges peut s'avérer justifiée pour deux raisons. Premièrement, les obstacles aux échanges de services ne sont pas discriminatoires à l'encontre des seuls prestataires étrangers : ils peuvent également entraver l'entrée sur le marché de nouvelles entreprises nationales. Il se peut que ce type de restrictions à l'accès au marché réduise les services d'origine nationale tout autant que ceux venant de l'étranger. Les méthodes axées sur l'évaluation quantitative de ces échanges transfrontières pourraient négliger de prendre en compte le premier aspect. Deuxièmement, dans certains secteurs de services, dont les télécommunications, le commerce transfrontières est peu important. L'essentiel des échanges s'effectue par le biais d'une présence commerciale; or, cette dernière ne figure tout simplement pas dans les statistiques conventionnelles relatives au commerce. Lorsque la présence commerciale est nécessaire, la production ou la consommation totales sont plus proches du concept quantitatif correspondant que le total des échanges.

\section{Mesures fondées sur les prix}

191. Jusqu'à ces derniers temps, on jugeait trop difficile de mesurer les incidences des obstacles au commerce des services sur les données variables que sont les prix ou les coûts. Ce point de vue s'explique principalement par la difficulté d'identifier les restrictions entravant les services et par la complexité du calcul d'un élément-témoin, c'est-à-dire du prix qui serait pratiqué en l'absence de barrières ${ }^{19}$. Deux

Il convient toutefois de noter que ces taux concernant la restrictivité des obstacles aux échanges et les politiques en vigueur sont plus élaborés que les variables traditionnelles, qui s'appuient sur des valeurs allant de 0 à 1 .

Depuis 2002, l'OCDE fournit des données bilatérales sur les échanges de services. Toutefois, le processus étant encore relativement récent, ces données font l'objet de certaines limitations [Source: voir www.oecd.org/std/trade-services]. L'OCDE les a utilisées récemment pour évaluer les incidences des obstacles réglementaires sur les échanges bilatéraux.

Dans le contexte des échanges portant sur des produits homogènes, les estimations relatives aux barrières fondées sur les prix s'appuient sur les écarts entre prix intérieurs et extérieurs. L'hypothèse sous-jacente est alors la suivante: dans un marché libre d'accès, les prix sont égaux aux coûts marginaux. Lorsque le produit national et le produit étranger peuvent se substituer parfaitement l'un à l'autre, le prix étranger «sortie d'usine» constitue une référence concrète pour déterminer ce que serait le prix intérieur en 
méthodes ont été proposées : Francois et Hoekman (1999) ont conçu un système de mesure fondé sur les marges d'exploitation brutes. Celles-ci donnent une indication de la rentabilité relative de différents secteurs et, à partir de là, de l'importance des différents obstacles à l'entrée sur le marché qui sont susceptibles d'exister. La deuxième méthode, élaborée par l'OCDE (2001), évalue l'impact des différentes mesures réglementaires sur les indicateurs de performance pour un certain nombre de secteurs de services. Les mesures relatives aux performances varient, en fonction des incidences que les barrières sont censées avoir (création de rentes ou augmentation des coûts).

192. Cette dernière méthode a également été perfectionnée par d'autres chercheurs en vue d'évaluer les effets des régimes réglementaires sur les prix, pour différents secteurs et dans un certain nombre de pays en développement ou développés (voir, en particulier, les travaux menés en collaboration par l'Australian Productivity Commission et l'Australian National University). L'équipe australienne a obtenu des résultats basés sur les prix pour des secteurs spécifiques: Trewin (2001) et Warren (2001) pour les télécommunications, Kalirajan et al. (2001) pour les services bancaires, Kang (2001) pour les services maritimes, Kalirajan (2001) pour la distribution de produits alimentaires, Nguyen-Hong (2001) pour les services professionnels (ingénieurs), Doove et al. (2001) pour le transport aérien, les télécommunications et l'électricité.

193. Les études susmentionnées proposent des méthodes permettant de mesurer l'ampleur des restrictions et leur impact sur les prix ou sur la production au niveau sectoriel. Toutes calculent des taux de restriction pour les secteurs considérés, ainsi que des systèmes de pondération visant à refléter, de manière subjective, les incidences économiques probables des différentes restrictions. L'aspect essentiel à souligner est le suivant : si les composantes qui ont permis d'établir un taux sont considérées individuellement, elles peuvent être introduites séparément dans le modèle économétrique, de sorte que la modélisation puisse contribuer à l'évaluation des facteurs de pondération. Elles font en outre la distinction entre les obstacles frappant le commerce transfrontières, la présence commerciale ou les mouvements de personnes (différenciation modale). On trouvera ci-après une brève synthèse des études qui modélisent séparément différents pays en développement. Cette synthèse s'inspire en partie de travaux antérieurs de l'OCDE.

\section{Télécommunications}

194. Trewin calcule et décompose les différences de coût observées dans le secteur des télécommunications d'après un échantillon de 13 pays à faible revenu et de 24 pays à revenu élevé, et constate l'existence de différences structurelles entre les deux groupes de pays. Par ailleurs, Warren a évalué les effets des obstacles au commerce et à l'investissement dans les services de télécommunications (téléphonie mobile ou fixe). Selon lui, les estimations pour les pays industrialisés avancés sont assez basses par rapport à celles, bien plus élevées, calculées pour les pays en cours d'industrialisation. Il a constaté que l'effet-prix des restrictions applicables aux prestataires étrangers de services de

l'absence de restrictions aux échanges. Par conséquent, l'écart entre les prix représente une évaluation pertinente des incidences de ces restrictions sur les coûts. Par contre, les services sont différenciés, non seulement en fonction des fournisseurs, mais aussi, pour chaque fournisseur, selon les différents consommateurs. On ne saurait partir de l'hypothèse que le prix étranger constitue la référence pertinente. Par contre, l'élément-témoin, c'est-à-dire le prix qui serait appliqué en l'absence d'obstacles aux échanges, doit être défini à partir d'un modèle économétrique de ce qui détermine le prix intérieur dans chaque économie. Les estimations relatives à ces modèles utilisent souvent des données portant sur plusieurs pays, mais la variable dépendante est le prix (ou l'écart prix-coût) à l'intérieur de chacun des pays, et non l'écart entre prix intérieur et prix extérieur. Par ailleurs, les secteurs de services se caractérisent par une concurrence imparfaite, des produits différenciés et des coûts croissants. Les marges prix-coûts seront positives, même en l'absence d'obstacles aux échanges, mais seront plus faibles s'il n'y a pas d'obstacles aux échanges. Les modèles économétriques s'appuient sur les variations entre pays pour évaluer l'importance de cette différence. 
télécommunications était inférieur à 20 pour cent dans la majorité des économies considérées. En la matière, les pays les plus restrictifs sont l'Indonésie, la Colombie, les Philippines, l'Afrique du Sud, la Thailande et la Turquie (l'effet sur les prix y oscille entre $20 \%$ et plus de $100 \%$ ). Les moins restrictifs sont les États-Unis, le Danemark, les Pays-Bas, la Nouvelle-Zélande, l'Australie, l'Allemagne et la Suède. On constate que les obstacles à l'accès au marché — qui concernent aussi bien les prestataires nationaux qu'étrangers - ont pour effet une majoration des prix qui peut atteindre $70 \%$.

\section{Services bancaires}

195. Kalirajan et al. ont évalué l'effet-prix des restrictions non prudentielles touchant aux marges d'intérêt de 294 banques dans 27 économies de la région Asie-Pacifique, d'Europe et d'Amérique. Le taux de restriction est calculé comme étant l'écart entre le taux prêteur d'une banque et le taux de rémunération des dépôts (ou coût des financements). S'appuyant sur une méthode économétrique en deux temps, Kalirajan et al. ont distingué les effets de règles prudentielles propres au secteur bancaire (ratios de fonds propres, réserves obligatoires ou contraintes de liquidités et charges d'exploitation nettes hors intérêts) d'autres facteurs tels que la volatilité des taux d'intérêt, la structure du marché et le taux de restriction des échanges. Ils ont constaté que les restrictions commerciales non prudentielles appliquées de manière discriminatoire aux banques étrangères augmentent de 5 à $60 \%$ le prix, ou la " marge d'intérêt nette », des différents services bancaires. C'est en Indonésie, aux Philippines, en Malaisie, au Chili, à Singapour, en Corée du Sud et en Thaïlande que l'impact sur les prix des restrictions imposées aux établissements étrangers est le plus important. En revanche, les règles non prudentielles imposées aux banques étrangères sont relativement peu importantes en Argentine, en Australie, au Canada, dans l'Union européenne, à Hong Kong Chine, en Suisse et aux États-Unis. Les obstacles qui entravent l'accès au marché - et affectent identiquement les banques nationales et les banques étrangères — ont un effet plus modeste sur les prix des services bancaires (de 0 à $24 \%$ ).

\section{Services maritimes}

196. Kang a utilisé les indicateurs de mesures restrictives (indices de fréquence chez McGuire) pour estimer l'impact de mesures restrictives (accès au marché et traitement national) sur les marges des échanges de services maritimes bilatéraux. Il a constaté que les restrictions imposées par les pays exportateurs ont généralement un impact beaucoup plus fort que celles pratiquées par les pays importateurs. Sachant que les pays à faible revenu ont des marchés moins libres d'accès, il semble qu'ils aient davantage à gagner que les pays à revenu élevé en éliminant les restrictions qui frappent les services maritimes.

\section{Distribution de produits alimentaires}

197. Kalirajan a mesuré les effets des entraves aux échanges de services de distribution dans 38 pays développés et en développement sous la forme de l'effet-coût sur le droit d'établissement. C'est en Belgique, en France, en Malaisie et en Suisse que sont relevées les restrictions les plus sévères, les coûts des entreprises s'en trouvant augmentés de 5 à $8 \%$. Ces économies se caractérisent par des entraves à l'établissement pouvant prendre la forme de restrictions à l'entrée de capitaux étrangers, aux fusions et aux acquisitions, ou à l'acquisition de terrains à usage commercial. Les pays les plus libéraux sont l'Australie, la Grèce, Hong Kong Chine, la Nouvelle-Zélande, Singapour et l'Afrique du Sud, avec une majoration des coûts inférieure à $1 \%$ et quelques restrictions sur les mouvements permanents de personnes et l'investissement étranger. Enfin, les pays qui se situent à un niveau intermédiaire sont le Canada, le Chili, l'Indonésie, l'Irlande, le Japon, les Pays-Bas et les États-Unis. 
Services professionnels (ingénierie)

198. Nguyen-Hong a calculé les incidences sur les prix et les coûts des restrictions imposées aux sociétés de services d'ingénierie dans 20 pays d'Asie-Pacifique, d'Europe et d'Amérique. Les répercussions, tant sur les prix que sur les coûts, peuvent dans l'ensemble être qualifiées de faibles à modérées, signe d'un environnement commercial relativement ouvert dans un grand nombre de pays pour ce qui concerne les services d'ingénierie. S'agissant des effets sur les prix, qui se répercutent principalement sur le droit d'établissement, c'est en Autriche, au Mexique, en Malaisie, en Indonésie et en Allemagne qu'ils sont les plus marqués, et en Afrique du Sud, aux Pays-Bas, en Australie et dans la plupart des pays européens qu'ils sont les plus faibles. Les effets les plus importants sur les coûts, essentiellement liés aux exigences nationales en matière de qualifications et aux restrictions sur la création d'entreprises et l'investissement non professionnel, ont été relevés en Autriche, en Malaisie et aux Pays-Bas. Dans toutes les autres économies, ils sont très faibles (moins de $5 \%$ ).

\section{Transport aérien et électricité}

199. Doove et al. (2001) se sont employés très tôt à quantifier les effets des régimes réglementaires nationaux en termes d'augmentation des coûts des échanges internationaux dans les domaines du transport aérien de passagers, des télécommunications et de l'approvisionnement en électricité. La démarche adoptée dans leur étude consiste à comparer les effets des réglementations en vigueur dans différents pays de la région Asie-Pacifique, d'Europe et d'Amérique avec ceux des régimes dont l'OCDE (2000) suppose qu'ils limiteraient les prix dans les secteurs de services pertinents. Doove et al. utilisent les indices réglementaires et les estimations économétriques de l'OCDE pour évaluer l'impact des restrictions sur les prestations de services dans les domaines du transport aérien et de l'approvisionnement en électricité, et appliquent les résultats aux pays en développement comme aux pays développés. Si l'indice proposé pour le secteur des télécommunications doit encore être affiné, ceux qui concernent le transport aérien et la fourniture d'électricité livrent déjà de précieuses indications.

200. L'impact sur les prix des restrictions appliquées dans le secteur du transport aérien varie selon les régions économiques et peut représenter de 3 à $22 \%$ pour les tarifs réduits. Il est relativement plus élevé dans les pays de la région Asie-Pacifique, où il se situe entre 12 et $22 \%$, en raison de réglementations plus strictes sur le transport de passagers et d'une libéralisation encore limitée. Les chiffres oscillent entre 11 et $18 \%$ dans les économies d'Amérique du Sud, tandis que l'Europe et l'Amérique du Nord affichent l'effet-prix le plus faible.

201. Dans le secteur de l'énergie, comme dans celui des télécommunications, les prix réagissent aux évolutions, même faibles, dans la valeur de certaines variables. Il n'est donc guère surprenant que ce soit dans les pays dont la réforme du secteur de l'approvisionnement en électricité est la plus avancée que les prix sont les moins touchés. Il s'agit notamment des pays d'Europe du Nord, du RoyaumeUni, de l'Australie, de la Nouvelle-Zélande et, parmi les pays en développement, de l'Argentine, du Chili et de la Colombie.

III. Éventail des résultats et méthodologie de base pour l'élaboration de modèles calibrés du commerce international

202. Les estimations portant sur les répercussions de la libéralisation à l'échelle d'une économie s'effectuent le plus souvent au moyen de modèles calibrés définis pour les échanges internationaux. Ces modèles sont dits «calibrés » ou «calculables » parce qu'ils supposent l'existence de formes explicites pour les fonctions de demande et d'offre, ce qui permet de résoudre numériquement les valeurs d'équilibre des prix et des quantités dès lors que le modèle correspond à un ensemble de données. Les valeurs de paramètres de ces modèles sont généralement choisies parmi les estimations existantes des obstacles aux 
échanges, le modèle étant résolu à partir de la théorie retenue en matière de commerce international. Les résultats sont donc fortement tributaires de la qualité des estimations relatives au flux des échanges et aux obstacles, ainsi que du type de représentation choisi pour le commerce international. Les modèles peuvent être «d'équilibre partiel » ou « d'équilibre général ». [voir l'Appendice 2]

203. Les modèles calibrés — dans lesquels il convient de voir un complément à la théorie — nous aident à déterminer les types de forces susceptibles de jouer un rôle important et à comprendre comment différentes forces peuvent parfois interagir de manière surprenante. C'est davantage la représentation qu'ils donnent de ces forces et de ces relations globales qui fait leur intérêt, que les chiffres obtenus sur tel ou tel pays.

204. La seule analyse publiée sur l'équilibre partiel semble être celle de Johnson et al. (2000). Les auteurs s'emploient à évaluer les effets de la concurrence dans le secteur des services de transport aérien, ainsi que les conséquences pour le bien-être de la création d'une alliance ouverte entre l'Australie, la Chine, Hong Kong Chine et le Japon. A une exception près, les estimations montrent que l'entrée d'un nouveau transporteur aérien australien sur les liaisons avec d'autres pays asiatiques a permis d'accroître le surplus et le bien-être des consommateurs australiens et étrangers. Les gains relatifs au bien-être sont passés de $28.4 \%$ en 1995 à $32.4 \%$ en 1997 pour l'Australie, contre $12.4 \%$ en 1995 et $31.7 \%$ en 1997 pour les autres pays d'Asie (Chine, Hong Kong Chine, Indonésie, Japon, Malaisie, République de Corée, Singapour, Taipeh chinois et Thaïlande).

205. Les analyses consacrées aux modèles d'équilibre général reposent généralement sur l'un des modèles suivants :

- Le Michigan Model of World Production and Trade, conçu de manière à prendre en compte des caractéristiques de la Nouvelle théorie commerciale : concurrence imparfaite, accroissement des rendements d'échelle et différenciation des produits - (voir Brown et al. (1996), Chadha (2000), Chadha et al. (2000) et Brown et Stern (2001).

- Le modèle GTAP, modèle d'équilibre général calculable, multisectoriel et multirégional, http://www.gtap.agecon.purdue.edu/products/ images/model.gif, tablant sur une concurrence parfaite et des rendements d'échelle constants - voir Hertel et al. (1999), Ministère australien des affaires étrangères et du commerce (1999) et Robinson et al. (1999).

- Le modèle FTAP, version élargie du modèle GTAP, conçue expressément pour tenir compte de la présence commerciale au plan bilatéral - voir Dee et Hanslow (2000) et Verikios et Zhang (2001).

- Le cadre APG (Asia Pacific G-Cubed), fondé sur une optimisation intertemporelle dynamique, qui inclut les interactions du secteur réel et du secteur financier - voir Ministère australien des affaires étrangères et du commerce (1999) et McKibbin et Wilcoxen (1996).

206. Jomini et al. (2002), Brown et Stern (1999) et une étude antérieure de l'OCDE évaluant les conséquences sur le bien-être national des obstacles au commerce de services (TD/TC/WP(2000)24/FINAL) fournissent un examen approfondi de ces modèles et de leurs caractéristiques principales, telles que l'ampleur, l'origine et la couverture sectorielle des obstacles initiaux. Nous présentons ici un simple résumé des travaux qui modélisent séparément les économies en développement. Il importe de rappeler que les écarts entre les différentes estimations des avantages d'une libéralisation des échanges de services sont liés principalement à l'importance accordée aux obstacles au départ. On trouvera en Appendice des tableaux comparatifs des résultats. 


\section{Études modélisant les services sous la forme d'échanges transfrontières (mode 1 de fourniture de services)}

- Brown et al. (1996) simulent les effets d'une réduction de $25 \%$ des équivalents tarifaires ad valorem des obstacles aux échanges de services, et s'appuient pour ce faire sur différentes hypothèses concernant les types de barrières, leur importance relative et la concurrence. Ils concluent que la libéralisation favorise le commerce pour tous les pays. S'agissant du PIB, c'est au Mexique (2\% du PIB), en Australie (1.9\%) et dans les nouveaux pays industriels (NPI) d'Asie $(1 \%)$ que le gain de bien-être est le plus élevé. Toutefois, les termes de l'échange varient légèrement en faveur des NPI d'Asie, de l'UE et des États-Unis, et au détriment du Canada, du Mexique, du Japon, de l'Australie et de pays répertoriés comme nouveaux pays industriels non asiatiques. Cette étude utilise les estimations approximatives de Hoekman (1995) concernant les équivalents tarifaires.

- Chadha (2000) étudie les effets d'une réduction de $25 \%$ de l'équivalent tarifaire ad valorem pour les secteurs des services. Les données qu'il utilise portent sur trois pays et régions d'Asie en développement (Inde, reste de 1'Asie du Sud-Est, ANASE-4 et NEI-4) et sur trois pays/régions développés (Union européenne, Japon, États-Unis). Les estimations font état d'une amélioration du bien-être assez importante, relativement plus élevée dans les pays d'Asie en développement que dans les pays développés. Les conséquences pour le bien-être de la libéralisation des échanges de services se traduisent par une évolution de $0.7 \%$ en Inde, de $0.9 \%$ dans le reste de l'Asie du Sud-Est (notamment au Bengladesh, au Bhoutan, aux Maldives, au Népal, au Pakistan et au Sri Lanka), de $1.8 \%$ pour l'ANASE-4 (Indonésie, Malaisie, Philippines et Thaïlande) et de $1.7 \%$ pour les quatre NPI (Hong Kong Chine, Singapour, Corée du Sud et Taipeh chinois). Parmi les développés, les États-Unis affichent une amélioration représentant $1 \%$, le chiffre étant de $0.8 \%$ pour l'UE et le Japon.

- Chadha et al. (2001) étudient les incidences d'une réduction de $33 \%$ des équivalents tarifaires définis par Hoekman (1995) pour les obstacles aux échanges de services. Ils fondent leurs travaux sur des données relatives à 15 pays en développement et 5 pays développés. Le gain de bien-être est estimé à $2.5 \%$ pour les pays en développement, contre $2 \%$ pour les pays développés. C'est dans les pays suivants qu'une libéralisation multilatérale semble générer les gains relatifs les plus importants : Hong Kong Chine (8.3\%) et Thaillande (4.2\%), suivis par Singapour, les Philippines, le Mexique et la Turquie (tous entre 3 et $4 \%$ ), le Sri Lanka, la Corée du Sud, l'Indonésie, les pays d'Europe centrale et les pays d'Amérique centrale et d'Amérique du Sud (plus de $2 \%$ ), l'Inde, la Chine, la Malaisie et le reste de l'Asie du Sud-Est (plus de $1 \%$ ). En ce qui concerne les pays développés, les gains s'échelonnent de $2.9 \%$ (Australie et NouvelleZélande) à $1.9 \%$ (Japon et Europe). Le cas de l'Inde mérite d'être souligné, puisque ce pays enregistre un accroissement du bien-être de $1.6 \%$ en cas de libéralisation multilatérale et de $1.3 \%$ dans un scénario de libéralisation unilatérale. Une libéralisation globale entraînerait une amélioration plus forte qu'une libéralisation limitée aux services.

- Benjamin et Diao (2000), qui se fondent sur la base de données GTAP (Global Trade Analysis Project) dans un scénario de concurrence monopolistique, modélisent la libéralisation des échanges dans onze secteurs de services en supprimant la segmentation du marché et en réduisant les coûts fixes de $10 \%$. Leurs estimations portent sur les gains de bienêtre dans les pays en développement suivants: Mexique et Chili (gains allant de 0.5 à $1.87 \%$ ), Hong Kong Chine (de 0.98 à $5.99 \%$ ), Taipeh chinois (de 0.61 à $10.80 \%$ ), Chine (de 0.02 à $7.33 \%$ ), reste de l'Asie du Sud-Est (de 0.91 à $7.72 \%$ ). Parmi les pays développés, c'est en Australie que l'amélioration est la plus nette $(2.95 \%)$ et c'est l'Union européenne qui enregistre la plus faible $(0.19 \%)$. 
- Le Ministère australien des affaires étrangères (1999), à partir du modèle et de la base de données GTAP couvrant 45 régions et 50 secteurs de services, et du modèle dynamique APG portant sur 18 régions et six secteurs, simule les effets de deux scénarios possibles : $50 \%$ et $100 \%$ de réduction des distorsions du niveau de protection des échanges de services. Les gains de bien-être pour l'Afrique, l'Europe orientale et l'Amérique latine atteignent 4.7 milliards d'USD, 7.7 milliards d'USD et 12.6 milliards d'USD respectivement. On dispose également de résultats concernant certains pays en développement : la Chine, l'Inde et la Corée du Sud, dont les gains représentent 9.8 milliards d'USD, 2.6 milliards d'USD et 3.9 milliards d'USD respectivement.

- Robinson et al. (1999) simulent plusieurs scénarios faisant intervenir une évolution de la politique. Ils constatent que, dans l'ensemble, les gains de bien-être obtenus par suite d'une réduction de $50 \%$ de la protection dans les secteurs des services sont cinq fois plus importants que ceux qui résultent d'une réduction similaire dans les échanges de produits. Par ailleurs, lorsque les transferts de technologie résultant des importations sont pris en compte, le bien-être s'accroît sensiblement. Quatre types d'évolution font l'objet d'une évaluation : (a) libéralisation des échanges de services sans transfert de technologie ; (b) libéralisation des échanges de services avec transfert de technologie; (c) libéralisation des échanges de services et de produits sans transfert de technologie ; (d) libéralisation des échanges de services et de produits avec transfert de technologie. Les estimations concernant les gains de bien-être portent sur les pays et régions en développement ci-après : NPI d'Asie (de $1.85 \%$ dans le cas d'une libéralisation des échanges de services sans transfert de technologie à $7.87 \%$ pour une libéralisation globale avec transfert de technologie), Chine (gains allant de 0.34 à $3.14 \%$ ), Asie du Sud-Est (de 1.13 à $3.14 \%$ ), Amérique latine (de 0.98 à $2.45 \%$ ). Les gains sont extrêmement divers dans les pays développés, mais les schémas correspondent à ceux des pays en développement.

- McKibbin et Wilcoxen (1996) n'utilisent pas d'estimation spécifique des obstacles aux échanges de services ; seuls les changements intervenant de façon exogène dans la productivité totale des facteurs sont pris en compte. Les auteurs axent leurs travaux sur les ajustements prévus entre 1995 et 2020 dans un grand nombre de pays de l'APEC, pour faire suite à la Déclaration de Bogor de 1994, qui proposait l'instauration du libre échange d'ici à 2020 dans la région AsiePacifique. L'un des aspects essentiels de l'étude concerne l'incidence des flux de capitaux internationaux, des prévisions et de l'accumulation de capitaux matériels sur l'estimation du volume et de la répartition des recettes supplémentaires que devrait générer cet ambitieux programme de réforme des échanges commerciaux. Le document compare les effets respectifs d'une libéralisation totale des échanges dans les pays de l'APEC, d'une libéralisation préférentielle entre les membres de l'APEC et d'une libéralisation limitée aux économies membres de l'ANASE. Il ressort de cette analyse que les économies participantes ont davantage à gagner d'une libéralisation totale non préférentielle.

- Francois et al (2002) ont évalué les avantages économiques globaux du Cycle de Doha, caractérisé par une structure entrées-sorties qui lie clairement les secteurs dans une chaîne de valeur ajoutée dès le stade des biens primaires, tout au long des différentes étapes intermédiaires, jusqu'à l'assemblage final des biens et services avant leur consommation. Les services apparaissent comme une source importante de gains, dont le montant est évalué à plus de 50 milliards d'USD à l'échelle mondiale. C'est aux États-Unis et en Inde que ces gains sont les plus élevés.

- La Banque mondiale (2002) a simulé un programme quinquennal de changements d'ordre politique devant conduire à l'élimination progressive des taxes à l'importation, des subventions aux exportations et des subventions nationales à la production. C'est ainsi que, de 2005 à 2010, les obstacles aux échanges seraient réduits chaque année de 1/6e par rapport à leur niveau initial. 
D'après cette étude, une réduction des barrières aux échanges de services capable de stimuler le commerce transfrontières et de réduire de $10 \%$ non seulement les pratiques inefficaces dues à la protection des monopoles dans le domaine des services, mais aussi les augmentations de prix inhérentes à la concurrence imparfaite, devrait accroître, d'ici à 2015, les revenus des pays en développement de 900 milliards d'USD (soit 9.4\%) par rapport aux niveaux de 1997.

\section{Études dont la structure de modélisation traite séparément les flux d'IDE, les activités de filiales étrangères et les mouvements temporaires de personnes (modes 3 et 4 de fourniture de services)}

207. Plusieurs études ont été consacrées récemment aux avantages de la libéralisation des services fournis selon les modes 3 et 4 . Dans le cas du mode 3 (présence commerciale), la libéralisation insuffle une énergie supplémentaire à l'économie en éliminant les facteurs d'inefficacité grâce à une concurrence internationale accrue. Ainsi que le relève la Banque mondiale (2002) : «les services sont non seulement moins onéreux, mais aussi plus variés grâce à l'augmentation du nombre d'entreprises en activité dans un pays donné ». Le mode 4 (mouvements de personnes fournissant des services) demeure un mode essentiel de fourniture de services pour les pays exportateurs.

- Dans une étude de Dee et Hanslow (2000), la libéralisation est modélisée sous la forme d'une suppression des obstacles aux échanges de services. Les avantages varient alors suivant les économies considérées : c'est dans les économies où les obstacles sont les plus importants que les auteurs prévoient les gains les plus élevés, alors que les pays plutôt plus libéraux devraient enregistrer des gains plus limités voire, dans certains cas, des pertes. Les gains dus à la libéralisation des échanges de services atteignent $14.6 \%$ du PIB en Chine, $5.1 \%$ en Indonésie, $0.7 \%$ en Malaisie, $0.4 \%$ aux Philippines et au Chili, $0.2 \%$ en Thailande et au Taipeh chinois et $0.1 \%$ dans les autres pays d'Amérique du Sud (Brésil, Argentine, Colombie et Uruguay). Cette étude impute les pertes de bien-être à des transferts de rentes ou aux effets des termes de l'échange. Les pays où les sorties d'IDE sont importantes enregistrent plutôt une régression du bien-être : en effet, la réduction des obstacles aux échanges dans les pays hôtes s'accompagne d'une diminution des rentes. Il importe cependant de souligner que les restrictions au commerce de services ne sont pas toutes créatrices de rentes. Lorsque les pays sont en mesure de baisser le coût des ressources, les gains s'accumulent de façon plus directe et les effets de redistribution des rentes sont moins importants. Dee et Hanslow emploient un système complexe de mesure des obstacles ainsi qu'une version actualisée du modèle GTAP, avec modélisation explicite de la mobilité du capital et de l'IDE, et, pour tous les secteurs, concurrence imparfaite, rendements d'échelle croissants et concurrence monopolistique de grands groupes (modèle FTAP).

- Verikios et Zhang (2001), qui s'inspirent de la structure de modélisation de Dee et Hanslow (2000), fournissent une analyse sectorielle plus détaillée et décomposent les avantages de la libéralisation en deux catégories : ceux qui découlent d'un accès aux marchés plus facile et ceux qui sont liés au traitement national. La simulation d'une libéralisation totale dans les secteurs des télécommunications, des finances, des assurances et des services aux entreprises se traduit pas des effets positifs en matière de bien-être. La répartition des avantages globaux entre les régions varie selon les scénarios politiques envisagés. Une libéralisation complète des échanges de services dans le domaine des télécommunications entraîne un gain de bien-être pour toutes les régions, à l'exception de la Malaisie, de la Thaïlande et du Mexique. Dans le cas des services financiers, les conséquences sont positives pour la plupart des économies. Les plus grands bénéficiaires sont les pays d'Asie du Sud-Est et d'Amérique latine. La libéralisation du traitement national permet à toutes les régions qui investissent un meilleur accès aux marchés étrangers. Les régions dans lesquelles les barrières discriminatoires et les entrées d'IDE sont importantes pourraient donc se trouver pénalisées (par exemple : l'Indonésie en ce qui concerne les services de télécommunications). Toutefois, la plupart d'entre elles ont tout à gagner 
d'une suppression des obstacles à la pénétration des marchés. On soulignera par ailleurs que les gains découlant d'une libéralisation intégrale tendent à être plus importants que la somme des gains produits par les deux scénarios de libéralisation partielle dans le cas de régions à obstacles élevés (généralement des pays en développement), et moins importants pour les régions où la situation est plus souple au départ (pays développés le plus souvent). Cela s'explique par le fait que, contrairement à la libéralisation intégrale, une libéralisation partielle laisse en place certains obstacles, susceptibles d'être générateurs de rentes.

- Brown et Stern (2001) modélisent la libéralisation des échanges de services sous la forme d'une réduction de $33 \%$ de l'équivalent ad valorem des obstacles, fondée sur le calcul des marges brutes d'exploitation des entreprises de services. Les données concernant les investissements étrangers reprises dans cette étude proviennent de l'Australian Productivity Commission. Des gains de bien-être apparaissent dans les pays qui attirent des capitaux matériels, quel que soit leur niveau de développement. Dans ces pays, les entrées de capitaux sont liées à une expansion de la production dans la plupart, voire dans la totalité, des secteurs de l'économie. Par ailleurs, les entreprises des secteurs en développement augmentent également leur production, réalisant ainsi des économies d'échelle. En revanche, les conséquences sur le bien-être dans les pays qui enregistrent des sorties de capitaux s'avèrent généralement négatives. Par conséquent, dans l'hypothèse d'une parfaite mobilité internationale des capitaux et d'un stock mondial de capitaux fixe, ce sont l'Indonésie, le Canada, la Nouvelle-Zélande et le Taipeh chinois qui enregistrent les gains les plus importants par rapport au PIB (15.6\%, $14.8 \%, 9.1 \%$ et $7.6 \%$ respectivement). Dans la plupart des pays et régions d'Asie, dont la Chine, Hong Kong, le Taipeh chinois, l'Indonésie, la Malaisie et les Philippines, les gains de bien-être se situent entre $1.7 \%$ et $6.6 \%$, dépassant ainsi les chiffres relatifs à l'UE $(0.5 \%)$, aux États-Unis $(0.5 \%)$ et à l'Australie $(1.8 \%)$. Certaines économies enregistrent des pertes : pays d'Amérique latine (entre -4.3 et $2 \%)$, la Corée du Sud $(-2.8 \%)$, la Thaïlande $(-2.2 \%)$ et le Japon $(-2 \%)$. Les termes de l'échange évoluent à petite dose en faveur de certains NPI d'Asie (Indonésie, Corée, Malaisie et Thaïlande), des pays d'Amérique latine, du Japon, de l'UE et des États-Unis, mais à l'encontre de l'Australie, du Canada, de la Chine, de Hong Kong, du Taipeh chinois, des Philippines et de Singapour.

- Konan et Maskus (2002), modélisant les gains de bien-être d'une libéralisation des échanges en Tunisie, étudient les écarts entre les gains selon que la libéralisation des échanges concerne les services ou les marchandises, le niveau et la composition de la production et les prix des facteurs dans une économie en développement. Les auteurs établissent un modèle d'équilibre général de l'économie tunisienne et modélisent la libéralisation des services sous la forme d'une suppression des restrictions sur la fourniture transfrontières de services et sur la participation étrangère par le biais d'IDE. Ils constatent qu'une libéralisation complète des services vaudrait à la Tunisie un gain de bien-être de plus de $5 \%$ (contre $1.5 \%$ pour la libéralisation du commerce de marchandises). L'essentiel de cette amélioration provient de l'ouverture des marchés dans les secteurs des services financiers, des services aux entreprises et des télécommunications.

- La méthodologie élaborée par l'Australian Productivity Commission afin de mesurer les obstacles au commerce des services a été utilisée dans le modèle conçu par Markusen et al (2001) pour évaluer les gains qui découleraient de la suppression d'une catégorie particulière de restrictions appliquées au mode 4 et portant sur les mutations internes aux entreprises. Cette étude examine la première utilisation faite de ce modèle, dans le cadre des travaux menés par Jensen, Rutherford et Tarr sur les conséquences, à l'échelle de l'économie entière, de l'accession de la Russie à l'OMC, en vue d'une analyse concrète des effets de la libéralisation dans un pays donné. Le modèle numérique innove, en ce qu'il reconnaît la nécessité des compétences étrangères pour permettre aux entreprises étrangère de se montrer compétitives dans le secteur 
des services nationaux. Les auteurs estiment que la suppression des obstacles à l'IDE dans les services explique environ 70 \% des gains de bien-être accumulés par la Russie depuis la libéralisation préconisée par l'OMC. En outre, ils situent entre 3 et $15 \%$ du PIB les gains obtenus par la levée des restrictions concernant les mouvements de personnel au sein des entreprises. L'un des résultats les plus intéressants de ce modèle, toutefois, réside dans le fait que le salaire réel de la main-d'œuvre spécialisée nationale augmente avec la libéralisation des politiques à l'égard des prestataires de services étrangers et que cette augmentation est proportionnelle au nombre d'entreprises étrangères sur le marché national.

- S'agissant du mode 4 de fourniture de services, Markusen et Rutherford (2002) ont étudié l'incidence de l'importation de compétences, rendue possible par la libéralisation de ce mode de prestation, sur le développement de l'esprit d'entreprise au plan national et sur la croissance intérieure. Leur analyse part du principe que le contact direct avec des experts étrangers — par exemple : ingénieurs-conseils ou conseillers en gestion - offre un moyen très important de transmettre des connaissances aux pays en développement ou aux économies de transition. L'étude montre en effet que le recours à des consultants étrangers peut avoir des répercussions considérables sur la croissance et le bien-être dans une économie nationale. Même si l'économie en question retrouve par la suite une courbe plus plate, elle conserve des revenus permanents plus élevés, qui s'ajoutent aux gains enregistrés pendant la période de transition. Les réformes permettant à un pays d'effectuer des emprunts internationaux à des taux favorables, ainsi que les retombées directes sous la forme d'une productivité accrue pour la masse des professionnels du savoir, amplifient les bénéfices engrangés sur le long terme.

- Toujours à propos du mode 4, Winters (2001), estime qu'une mobilité accrue de la main-d'œuvre internationale pourrait générer des gains annuels de plus de 300 milliards d'USD par an, à partir des hypothèses suivantes : un employé quittant un pays à faible revenu pour un pays à revenu élevé ne comble qu'un quart de la différence de productivité ou de salaire entre les deux pays; 50 millions de personnes supplémentaires originaires de pays en développement partent travailler à l'étranger au cours d'une année donnée, ce qui représente une augmentation d'environ $5 \%$ de la population des pays industrialisés; l'écart initial entre les salaires s'élève à 24000 USD par an.

- Dans d'autres études, Winters et Walmsley (2002) et Winters, Walmsley, Wang et Grynberg (2002) suggèrent qu'une hausse des quotas fixés par les pays développés sur les entrées de maind'œuvre en provenance de pays en développement, correspondant à $3 \%$ de la main-d'œuvre totale des pays développés, se traduirait par un gain global de 150 milliards d'USD par an, soit $0.6 \%$ du revenu mondial initial; les gains des pays développés comme ceux des pays en développement découleraient principalement des mouvements de personnes peu qualifiées (Winters et al., 2002). Toutefois, les auteurs reconnaissent que l'adaptation des salaires à la concurrence des travailleurs peu qualifiés des pays en développement a un coût social élevé, et ils proposent plusieurs pistes pour lutter contre ce phénomène (par exemple : une libéralisation progressive, programmes d'aides compensatoires à l'intention des travailleurs concernés). De même, Rodrick (2001) modélise la création d'un programme de visa de travail temporaire, assorti d'un quota correspondant à $3 \%$ de la main-d'œuvre des pays développés, selon lequel des personnes qualifiées et non qualifiées des pays en développement travailleraient dans des pays développés pour une durée de trois à cinq ans, avant de retourner chez elles et d'être remplacées par une nouvelle vague d'arrivants. Selon Rodrick, un tel système rapporterait 200 milliards d'USD chaque année. 


\section{Le rôle de la concurrence et de la structure des marchés}

208. Le modèle d'équilibre général comparatif (CGE) repose sur la conviction que les réformes visant à libéraliser les secteurs de services se traduiront par des structures de marchés plus compétitives. Toutefois, le remplacement des monopoles d'État par des monopoles privés - nationaux ou étrangers ne produit que des bénéfices négligeables dans la plupart des cas. Francois et Wooton (2001) étudient l'incidence de la structure des marchés, dans le secteur des transports, pour la répartition des gains générés par la libéralisation du commerce. Ils montrent que les répercussions sur le bien-être, dans trois macro-régions (Amérique latine, Asie du Sud-Est, Afrique australe et sub-saharienne), d'un accès entièrement libre aux marchés dans le secteur maritime dépend très largement du degré de concurrence dans le secteur du transport. Plus celle-ci est forte, plus importante est la part des gains liés à un meilleur accès au marché qui profite aux fournisseurs et aux consommateurs plutôt que de rester dans le cartel.

\section{IV. Études économétriques}

209. Afin de compléter la réflexion sur les études de modélisation concernant les gains de bien-être, le présent document examine différentes études économétriques centrées sur les effets de la libéralisation dans certains secteurs de services en termes de croissance économique et de taux de production. Les paragraphes ci-après présentent, dans leurs grandes lignes, les avantages potentiels du recours à une approche économétrique pour évaluer les conséquences à l'échelle de l'économie. Il est cependant admis que la plupart font l'objet de réserves importantes.

210. En vérifiant le bien-fondé de l'hypothèse selon laquelle la croissance, la production ou d'autres indicateurs de performance sont des aspects fonctionnels de la libéralisation économique, et en tenant compte des ajustements à long terme qui interviennent par le biais des accumulations de capitaux, de la croissance démographique ou du progrès technologique, ces études apportent une dimension dynamique importante aux modèles d'équilibre général, dont la majorité se limitent aux avantages statiques ponctuels de l'élimination ou de la réduction des obstacles aux échanges de services. ${ }^{20}$. D'une portée certes plus limitée, leurs prévisions n'en constituent pas moins un instrument précieux d'évaluation des effets de la libéralisation : en premier lieu, elles nécessitent peu d'informations au départ, ce qui limite l'impact sur les résultats en cas de données imprécises concernant les obstacles aux échanges; deuxièmement, les résultats de ces études s'appuient sur des données et sur des hypothèses relativement peu sujettes à caution (comme les répercussions positives d'une plus grande ouverture économique sur la productivité), plutôt que sur la validité supposée d'un vaste cadre théorique; enfin, la précision et la fiabilité des estimations économétriques sont plus faciles à juger, et les résultats parfois divergents de différentes études plus faciles à analyser au moyen de tests statistiques confirmés et de théories éprouvées.

211. La plupart de ces études, cependant, suscitent les critiques de Rodriguez et Rodrik, qui montrent que les régressions portant sur plusieurs pays ne permettent aucun contrôle sur la plupart des facteurs qui le justifieraient lorsqu'elles tentent d'isoler les effets des politiques commerciales, et surtout le taux

20 Les modèles EGC ne sont conçus ni pour prendre en compte les éventuels effets à long terme sur le taux de croissance (gains dynamiques) ni pour modéliser le lien entre réforme du commerce et productivité. Ce lien étant absent de leurs structures de modélisation, on considère souvent qu'ils ne reflètent que les gains statiques découlant de la réduction des sources d'inefficience économique liées aux distorsions de la politique commerciale. Dans la réalité, toutefois, certains avantages dynamiques découlant d'une évolution dans les investissements ou les structures apparaissent même dans les modèles EGC. En outre, les études EGC qui retiennent l'hypothèse d'une concurrence imparfaite comprennent au moins une source de croissance endogène -- l'impulsion endogène due à une plus grande diversification (à l'exemple de DixitStiglitz). On en trouve des illustrations chez Markusen, Rutherford et Tarr, dans Brown et Stern, et chez Dee et Hanslow. 
d'investissement ou le taux d'épargne. Qui plus est, lorsque des variables de contrôle satisfaisantes sont retenues, les liens économétriques devraient être vérifiés après un certain temps (par le biais d'analyses de séries chronologiques ou de coupes transversales). Rodriguez et Rodrik formulent une autre critique importante concernant la façon dont les politiques commerciales sont (ou, plus exactement, ne sont pas) évaluées dans un grand nombre d'analyses en régression de la croissance/de l'ouverture.

212. Les éléments économétriques disponibles sur le rôle des services comme données intermédiaires permettent de penser que l'ouverture du secteur des services influe sur la croissance à long terme. Cela confirme les prévisions des théories économiques, notamment la théorie de la croissance endogène et la nouvelle théorie commerciale, qui font état des avantages dynamiques qu'entraîne l'augmentation de la productivité et de la croissance obtenue au moyen de transferts de technologie, d'une efficience X, d'une plus grande diversité de l'offre et d'une amélioration de la qualité des services. Une productivité accrue dans les secteurs en aval, obtenue grâce à un meilleur accès à une gamme plus large de services, à une meilleure qualité et à des coûts à la production moins élevés (Rivera-Batiz et Rivera-Batiz 1992 et Markusen, 1989), est une source importante de gains dynamiques. D'après les modèles de croissance endogène, en revanche, il semblerait qu'en raison de l'un des traits caractéristiques du secteur des services - à savoir : la mobilité des facteurs de production - la croissance générée par le commerce des services est également renforcée si le pays qui a choisi de libéraliser ces échanges a un désavantage comparatif dans ce secteur (Mattoo et al., 2001) ${ }^{21}$. La mobilité des facteurs de production implique des mesures de libéralisation applicables sans distinction aux résidents et aux non-résidents, en modifiant la contestabilité d'un marché et, partant, son potentiel de croissance. Francois et Wooton (2000) ont appuyé cette thèse, estimant qu'il existe des liens importants entre le degré de concurrence, le mode et le degré d'accès au marché, et le rôle de la libéralisation en faveur de la concurrence.

\section{Services financiers}

213. De nombreuses études ont confirmé l'influence favorable sur la croissance d'une ouverture du secteur des services financiers — c'est-à-dire la thèse de Schumpeter selon laquelle le développement du secteur bancaire facilite la croissance économique par le biais du progrès technologique et de l'accumulation de capitaux.

$21 \quad$ Ainsi que l'indiquent Rodriguez et Rodrik (1999), l'impact de la libéralisation des échanges sur la croissance est lié à la question de savoir si les transferts de ressources s'effectuent à partir de secteurs n'ayant pas les caractéristiques nécessaires pour assurer la croissance à long terme vers des secteurs qui possèdent ces caractéristiques. Il s'agit principalement de la création de connaissances et d'externalités positives. Lorsque certaines conditions sont réunies (c'est-à-dire si la libéralisation des services réussit à mobiliser les ressources de secteurs ne possédant pas les caractéristiques nécessaires pour assurer la croissance à long terme vers des secteurs qui les possèdent, si ces secteurs permettent les transferts de technologie et s'ils ont la capacité de générer des effets d'échelle), on peut dire que la caractéristique particulière en cause du commerce de services est source de croissance. Cette caractéristique est la nécessité de la production et de la consommation simultanées de nombreux services faisant l'objet d'échanges, ce qui nécessite la mobilité des facteurs de production (capitaux et main-d'œuvre) afin de produire localement les «importations» de services (modes 3 et 4), ou celle du consommateur, qui utilisera à l'étranger les services « exportés » (mode 2). Il est donc probable qu'une libéralisation effective modifiera la contestabilité d'un marché et, par conséquent, son potentiel de croissance. Compte tenu des modifications sous-jacentes à la structure du marché, la croissance générée par les échanges de services se trouve renforcée si le pays engagé dans le processus de libéralisation a un désavantage comparatif dans le secteur concerné. Il n'en va pas ainsi pour le commerce des marchandises (Mattoo et al., 2001). 


\section{Travaux antérieurs}

214. Bien que la présente étude mette en évidence l'existence d'une relation étroite et importante entre l'évolution de la croissance et le degré d'ouverture des principaux secteurs de services, ces aspects sont, en réalité, à l'examen depuis beaucoup plus longtemps. Dans une étude antérieure déterminante, Goldsmith (1969) soulignait avec force le rôle des services financiers, grâce auxquels les investissements peuvent être orientés vers les utilisations les plus fructueuses, ce qui permet d'accroître production et revenus. Il voyait dans le rapport entre la valeur des moyens financiers intermédiaires et le PIB une variable représentant, d'une certaine façon, les performances du secteur financier à l'échelle d'un pays. Goldsmith s'en est servi dans une régression pour laquelle les taux de croissance économique constituaient la variable dépendante, et a constaté ce qu'il a appelé un "parallélisme global » entre la croissance et le niveau de développement financier représenté par cette variable. Les travaux qui ont suivi allaient tous dans le même sens que les études récentes, à savoir : l'ouverture et le degré de développement des secteurs de services sont liés à l'amélioration de la croissance.

215. King et Levine (1993) ont par la suite cherché à déterminer d'autres facteurs ayant un impact sur la croissance à long terme. Ils ont employé, dans leurs équations de croissance, d'autres mesures du développement d'un pays pour ce qui concerne le secteur financier, notamment le volume du passif dans le système financier rapporté au PIB et le ratio entre le passif du secteur privé et le PIB. Ils ont constaté que ces mesures jouaient un rôle important et supposaient une relation significative sur le plan économétrique. Cherchant à déterminer si le niveau de développement du secteur financier d'un pays en 1960 - mesuré à partir de l'un de leurs ratios - permettait de prévoir le taux de croissance de ce pays pour la période 19601990, ils sont parvenus à la conclusion que le degré de développement du secteur financier en 1960 constituait effectivement un bon indicateur de ce que serait la croissance économique par la suite.

216. Dans un document de 1997, Levine a fait une évaluation plus poussée du rôle des secteurs financiers dans le développement économique. Il s'est intéressé à cinq fonctions du secteur des services financiers: aide à la négociation du risque, affectation des capitaux aux usages les plus productifs, suivi des performances de gestion, mobilisation de l'épargne grâce aux innovations financières, et facilitation des échanges de biens et services.

\section{Travaux récents}

217. C'est plus récemment, toutefois, que le rôle des échanges et l'ouverture des secteurs de services, ainsi que leur influence sur la croissance, sont apparus avec plus de clarté. Mesurant l'impact de la participation de banques étrangères sur différents indicateurs de l'efficience bancaire d'un pays, Claessens et al. (2001) constatent que l'entrée de banques étrangères entraîne une efficience accrue du système bancaire d'un pays. Leurs conclusions s'appuient sur une estimation effectuée selon la méthode des moindres carrés, sur un échantillon de 7900 banques de 80 pays du monde entier, en tenant compte des effets fixes de pays et de temps. Les estimations s'accompagnent d'un contrôle de tous les facteurs financiers et macroéconomiques standard intervenant dans les relations étudiées.

218. Le lien entre efficience et croissance est explicitement modélisé et vérifié dans les travaux de Eschenbach et Francois (2002). Leur document présente et teste différentes voies par lesquelles les échanges de services financiers peuvent avoir des effets dynamiques favorables à la concurrence. Ils aboutissent à la conclusion suivante : indépendamment des autres éléments statistiques pertinents et toutes choses constantes par ailleurs, si un pays à faible revenu, représentatif de cette catégorie de pays, devait passer de la situation d'ouverture moyenne qui caractérise ces pays à un régime plus libéral, et donc plus ouvert, plutôt caracéristique des pays à revenu élevé (soit une libéralisation d'environ $50 \%$ d'après les mesures employées ici), le résultat indiquerait une différence de taux de croissance qui se situerait approximativement entre $0.4 \%$ et $0.6 \%$ par an et par habitant. 
219. Mattoo et al. (2001) ont testé une équation de croissance standard sur un échantillon de 60 pays répartis dans le monde entier, dont 37 en développement. Ils ont constaté que le taux de libéralisation du secteur financier était positif, voire important, et se situait à $5 \%$. Les pays ayant déjà en partie libéralisé le secteur des services financiers affichent une croissance de $0.25 \%$ plus rapide que celle des pays dans lesquels ce secteur est resté fermé. Pour les pays où la libéralisation est totale, la croissance est de $1.2 \%$ plus rapide que pour les autres. Par ailleurs, l'importance des effets de la libéralisation est beaucoup plus élevée pour les régressions portant exclusivement sur les 37 pays en développement que pour celles qui concernent l'échantillon complet (60 pays). Dans les pays en développement dont le secteur financier connaît une certaine libéralisation, la croissance tend à être de $0.36 \%$ plus rapide que ceux dont ce même secteur est fermé. Les pays en développement ayant totalement libéralisé les services financiers ont une croissance plus rapide de $2.3 \%$ que les autres pays en développement.

220. De même, Francois et Schuknecht (2000) ont construit des régressions de la croissance portant sur un échantillon de 93 pays : ils ont constaté que le lien était étroit et positif entre la concurrence dans le secteur financier et l'ouverture de ce même secteur, mais aussi entre croissance et concurrence. Cet élément est selon eux révélateur de l'existence d'une chaîne causale associant ouverture et concurrence, ainsi que concurrence et croissance. Compte tenu des effets nets de cet enchainement causal, le passage d'un régime fermé à un régime relativement ouvert dans le secteur des services financiers est en corrélation étroite avec d'importantes pressions en faveur de la concurrence et, finalement, avec l'obtention de taux de croissance élevés $(0.7 \%$ pour un pays en développement représentatif de sa catégorie).

\section{Télécommunications}

221. Les effets sur la croissance de la libéralisation des services de télécommunications peuvent être démontrés par l'existence d'un lien positif entre les performances enregistrées dans ce secteur et la croissance et par les répercussions positives de la libéralisation sur ces performances.

222. En dépit d'un intérêt politique manifeste, peu d'études examinent l'influence particulière des performances dans le secteur des télécommunications sur la croissance et le développement économiques ; toutes, cependant, soulignent la relation fortuite mais positive qui existe entre les performances enregistrées dans le secteur des télécommunications et la croissance économique. Boylaud et Nicoletti (2000) et l'OCDE (2001) aboutissent à la conclusion que la libéralisation — prévue et effective — des télécommunications a des effets bénéfiques sur l'efficacité du secteur, la qualité des services et les prix dans les pays de l'OCDE. Roller et Waveman (2001) constatent qu'il existe un lien positif entre l'infrastructure des télécommunications et la croissance économique d'un pays. Greenstein et Spiller (1996) ont cherché à définir l'impact de l'infrastructure des télécommunications (mesurée d'après le nombre de câbles en fibres optiques et de lignes RNIS) sur les performances économiques des États-Unis ; d'après les résultats de leurs travaux, une part non négligeable de l'augmentation de l'excédent de consommation enregistré dans le pays au cours des années 1990 est à porter au crédit de l'investissement dans cette infrastructure. Norton (1992) s'appuie sur des données relatives à 47 pays développés et en développement sur la période 1957-1977. Nonobstant certains problèmes d'endogénéité éventuellement constatés, son étude souligne que la quantité moyenne de téléphones constitue une variable indépendante, importante et positive en matière de taux de croissance annuel moyen d'un pays. Norton a en outre évalué les taux de croissance plus élevés que le Myanmar, le Honduras, Sri Lanka et la Bolivie auraient enregistré compte tenu des coefficients estimés et du taux de pénétration du téléphone pour le Mexique ou le Canada. Constatant que les impacts sont très élevés, il conclut qu'ils doivent s'expliquer par l'effet conjugué de la réduction des coûts de transaction, en fonction de l'infrastructure des télécommunications, et de la croissance de tous les autres secteurs, encouragée par le secteur des télécommunications. 
223. L'impact de la politique menée en matière de télécommunications sur les performances du secteur fait l'objet d'une étude de Fink, Mattoo, et Rathindran (2002). Ceux-ci abordent plus spécialement trois questions : premièrement, quelles répercussions certaines modifications de la politique commerciale - concernant la participation et la concurrence - ont-elles sur les performances du secteur ? Deuxièmement, en quoi l'impact de modifications apportées à une politique donnée peut-il être influencé par la mise en œuvre d'une autre politique, et par le cadre réglementaire global? Troisièmement, l'ordre de mise en oeuvre des réformes a-t-il une incidence sur les performances? Des données concernant 86 pays en développement d'Afrique, d'Asie et d'Amérique latine pour la période 1985-1999 montrent, selon eux, que la privatisation et la concurrence se traduisent toutes deux par une amélioration notable des performances. Un programme de réforme détaillé, prévoyant à la fois une évolution des politiques commerciales et le soutien d'un organe de réglementation indépendant, a conduit à une augmentation de $8 \%$ du nombre de lignes téléphoniques et à une hausse de $21 \%$ du niveau de productivité par rapport aux années où la réforme n'était que partielle ou inexistante. Il est intéressant d'observer que l'ordre de mise en oeuvre des réformes a une importance : la pénétration du téléphone est plus faible si la concurrence est postérieure à la privatisation au lieu d'intervenir simultanément.

224. Plus généralement, une étude de Mattoo, Rathindran et Subramanian (2001), déjà mentionnée dans le présent document, prévoit que les pays ayant commencé à libéraliser les services de télécommunications connaîtront une croissance de $0.15 \%$ plus rapide que les pays dans lesquels ce secteur est un monopole, où l'entrée d'IDE est interdite et où il n'existe pas d'organe de réglementation. Les pays dans lesquels la libéralisation des télécommunications est complète tendent à afficher unr croissance de $1 \%$ plus rapide que celle des autres. Le coefficient appliqué à l'indice de libéralisation des télécommunications est positif et élevé $(10 \%)$. Il laisse prévoir que les pays en développement ayant procédé à une certaine privatisation de leurs services de télécommunications ont une croissance de $0.32 \%$ plus rapide que celle des pays en développement dont les télécommunications se caractérisent par une situation de monopole, qui empêchent toute entrée d'IDE et qui ne disposent d'aucun organe de réglementation. Les pays en développement dans lesquels la libéralisation des télécommunications est totale ont une croissance de $2 \%$ plus rapide que celle des autres. Ces pourcentages sont deux fois plus élevés que ceux concernant l'échantillon complet de 60 pays.

\section{Transport maritime}

225. Fink, Mattoo et Neagu (2001) ont exploité une nouvelle base de données de la Banque mondiale pour tester un modèle économétrique des prix de transport de ligne. L'analyse économétrique, qui confirme l'importance de tous les déterminants standard des prix des services de transport, montre que les politiques officielles comme les pratiques privées exercent une influence considérable sur le prix des transports maritimes. ${ }^{22}$ Les restrictions appliquées à la fourniture de services portuaires font augmenter les prix de façon non négligeable, tandis que les politiques de réservation de cargaisons ${ }^{23}$ semblent pour la plupart sans effet. Ce sont toutefois les pratiques anti-concurrentielles de prestataires privés qui contribuent le plus au maintien de prix élevés. Alors que la libéralisation des échanges permettrait une réduction moyenne de $9 \%$ des prix des transports et une économie sur les coûts pouvant atteindre 850 millions d'USD, la suppression des accords passés entre transporteurs ferait baisser les prix de $25 \%$ et

22 Le terme «accords entre transporteurs privés » peut recouvrir une multitude de pratiques, certaines franchement anti-concurrentielles (comme la fixation de tarifs communs), d'autres probablement propices à une meilleure efficacité (comme le partage de couloirs de navigation et les consortiums).

23 La politique de réservation de cargaisons consiste à exiger qu'une partie de la cargaison transportée dans le cadre d'échanges commerciaux avec d'autres pays soit réservée à des navires battant pavillon national ou considérés comme «nationaux » selon d'autres critères. Les arguments avancés pour justifier ces politiques concernaient généralement la sécurité du pays (autonomie en périodes de guerre) ou les aspects économiques (industries naissantes). Il importe néanmoins de souligner qu'elles ne sont plus appliquées suite à la disparition de facto, ces dernières années, du Code des conférences maritimes de l'ONU. 
pourrait entraîner jusqu'à 2 milliards d'USD d'économies supplémentaires sur les seuls transports de marchandises à destination des États-Unis. Il est par ailleurs probable que les bénéfices indirects d'une réduction des entraves au commerce seraient également considérables. Ce document confirme les indications de l'étude - déjà mentionnée — de Francois et Wooton (2001) sur le rôle essentiel de la concurrence dans la libéralisation des services de transport.

226. Clark, Dollar et Micco (2001), au terme d'une analyse de l'efficacité portuaire comme déterminant des coûts de transport maritime, expliquent par un excès de réglementation l'inefficacité relative des ports d'Amérique du Sud. Si les exigences particulières imposées aux prestataires étrangers de services de manutention du fret ne semblent pas d'avoir d'incidence marquante sur les coûts, la pratique des services portuaires obligatoires pour les navires entrants est bénéfique à un faible niveau mais entraîne des effets négatifs à un niveau trop élevé. La réduction de l'efficacité portuaire imputable à des services portuaires obligatoires excessifs est estimée à $5 \%$. S'agissant de l'échantillon étudié dans ce document, l'Argentine semble avoir tiré parti du caractère modéré de la réglementation en vigueur dans ses ports maritimes; en revanche, les ports brésiliens, qui appliquent une réglementation excessive, enregistrent une baisse considérable d'efficacité.

\section{Conclusion}

227. Tant le sentiment général que les conclusions des études empiriques appuient l'idée selon laquelle les échanges de services restent soumis à des restrictions beaucoup plus fortes que le commerce des produits manufacturés, et de façon nettement plus marquée dans les pays en développement que dans les pays développés. Pourtant, les pays en développement forment en réalité un groupe extrêmement hétérogène, dont les membres présentent des caractéristiques très diverses et appliquent des restrictions très différentes en matière de fourniture de services.

228. Il existe peu de documents permettant de connaître avec précision l'ampleur de la segmentation des marchés de services dans les différents pays; nombreux sont ceux, toutefois, qui la jugent importante. Par exemple, en dépit des affirmations concernant une mondialisation toujours croissante, ce sont les banques locales qui continuent d'assurer la majeure partie des services bancaires dans la plupart des pays, les polices d'assurances sont toujours rédigées par des sociétés locales, et les liaisons aériennes intérieures restent assurées par les compagnies locales, tout comme le transport routier, ferroviaire et maritime. Aujourd'hui encore, les services de détail restent dominés par les prestataires intérieurs sur la plupart des marchés nationaux.

229. Dans les pays développés, certains éléments permettent d'affirmer que les échanges de services transfrontières sont en hausse. Cette évolution s'explique en partie par des conditions sous-jacentes au marché, engagements fermes pris en faveur d'une amélioration de l'accès au marché, ouverture, transparence et concurrence intérieure par exemple, et en partie par des causes plus immédiates: IDE effectués entre les pays, rachats de prestataires de services locaux, ou fusions avec des entités étrangères. L'intégration du marché semble donc progresser dans ces pays en ce qui concerne les secteurs de services, en dépit d'une évolution plutôt lente des réglementations.

230. Dans les pays en développement, en revanche, le processus d'intégration et d'interpénétration des marchés marque plutôt un ralentissement, bien qu'il soit en fait sur le point de se développer plus rapidement que dans les pays avancés. Pour le moment, la plupart des fournisseurs de services locaux demeurent des entités d'appartenance locale, et les échanges de services entre pays en développement sont limités, en partie à cause des obstacles imposés aux prestataires étrangers. Rapporté au commerce global, le commerce des services est également moins important dans les pays en développement que dans les pays développés. 
231. Toutes les études prises en compte montrent que, quels que soient le secteur analysé et la méthode employée, les obstacles au commerce sont en moyenne plus restrictifs dans les pays en développement que dans les pays développés.

- Les télécommunications ainsi que les secteurs bancaire et financier se caractérisent par des obstacles élevés dans les pays en développement et moins contraignants dans les pays développés.

- Dans un certain nombre d'autres secteurs (éducation, transport maritime, services professionnels et distribution), la différence entre pays en développement et pays développés est moins nette.

- De même, les mesures des obstacles fondées sur les prix ne font pas ressortir clairement l'existence d'une corrélation entre le niveau des obstacles estimés et les indicateurs du bien-être et de la production.

232. A ce jour, la plupart des études indiquent que la libéralisation des services procurera probablement des avantages importants aux pays dans lesquels les obstacles au commerce sont élevés au départ. Par conséquent, les pays en développement devraient être, à long terme, ceux qui tireront le plus d'avantages de la libéralisation des services. La plupart de ces avantages découlent de la libéralisation du secteur national des services, et non de la recherche d'un meilleur accès aux marchés de services étrangers. A court et à moyen terme, cependant, les avantages attendus pourront être atténués par les coûts d'ajustement en rapport avec l'élimination des obstacles aux échanges et la re-réglementation, qui représenteront sans doute une charge particulièrement importante pour les pays en développement.

- Les ajustements découlant de la libéralisation, tout en représentant un défi, pourront être facilités par l'adoption de structures appropriées; les faits montrent à ce propos que les coûts d'ajustement pourraient être moins élevés pour les échanges de services que pour les échanges de produits manufacturés. Dans ce contexte, il sera essentiel de prendre en considération la nature, le rythme et le déroulement du processus de libéralisation afin de contrôler les ajustements nécessaires et de faire en sorte que ce processus s'appuie sur des cadres réglementaires solides. La structure de l'AGCS, par sa souplesse intrinsèque et l'importance qu'elle accorde au caractère progressif de la libéralisation, traduit bien la nécessité d'un examen attentif de ces différents aspects. Pour de nombreux pays en développement, cependant, la mise en place de réglementations appropriées pour régir ce processus - ainsi que la possibilité d'en assurer l'application — nécessitera un renforcement considérable des capacités.

233. On a constaté en outre que les avantages de la libéralisation des échanges des services sont jusqu'à cinq fois supérieurs à ceux de la libéralisation des échanges de marchandises. Les estimations varient cependant en fonction de l'importance des obstacles initiaux, des cadres théoriques, des techniques de modélisation et des séries de données utilisées. Il ne paraît donc pas judicieux de considérer un chiffre ou une fourchette spécifique comme représentatif des avantages potentiels de la libéralisation des échanges.

- L'éventail complet des degrés initiaux d'intervention des pouvoirs publics trouve son reflet dans la diversité des effets de la libéralisation des services sur le bien-être obtenus avec des modèles d'équilibre général. Les études utilisant les estimations approximatives ("guesstimates ») de Hoekman pour les barrières initiales font généralement apparaître des gains de bien-être importants. Par contre, les études employant les estimations calculées à partir des mesures d'impact sur les prix ou les volumes donnent habituellement des gains de bien-être moins importants, quoique non négligeables. 
- L'ampleur des avantages tirés de la libéralisation dépend de la nature des obstacles et des autres restrictions qui pèsent sur les échanges. Lorsque les restrictions sont créatrices de rentes, elles font monter les prix au-dessus des coûts, créant ainsi des rentes dont bénéficient les producteurs concernés. L'élimination des restrictions créatrices de rentes peut alors se traduire par d'importants transferts des prestataires aux utilisateurs de services, et par un gain net relativement faible pour l'économie en général. En revanche, lorsque les restrictions se traduisent par une hausse des coûts, elles faussent les conditions du marché et sont responsables de modes de production inefficaces et coûteux. L'élimination des restrictions qui font monter les coûts peut être avantageuse pour les prestataires de services comme pour les utilisateurs, et représenter un gain relativement important pour l'économie en général. Elle peut donc être plus bénéfique que l'élimination des restrictions créatrices de rentes.

- On obtient des gains plus élevés, mais inégalement répartis selon les pays, lorsqu'on modélise explicitement la mobilité des capitaux et l'IDE et que l'on reconnaît le caractère imparfait de la concurrence. Il importe de signaler qu'apparemment les effets importants obtenus à l'aide de modèles ne découlent pas forcément de la libéralisation des échanges de services en soi, mais de la libéralisation supposée des marchés de facteurs qui l'accompagne. Cette observation est parallèle à celle qui concerne les travaux sur les échanges de marchandises, où la libéralisation des marchés de facteurs qui accompagne la libéralisation du commerce des marchandises produit parfois, au plan international, des effets importants et inégaux selon les pays.

- Les études qui traitent explicitement l'investissement direct étranger comme un mode de fourniture de services indiquent que la libéralisation des échanges de services augmente le revenu mondial global, mais que certains pays voient leur bien-être diminuer légèrement. Les pertes de bien-être peuvent avoir trois origines. Premièrement, la levée des restrictions à l'investissement étranger peut détourner les capitaux vers des pays qui, auparavant, avaient des obstacles à l'investissement relativement élevés. Deuxièmement, les obstacles à l'entrée génèrent des rentes, dont certaines vont aux détenteurs de capitaux étrangers. Avec la libéralisation, ces rentes sont érodées par la concurrence et les pays qui sont d'importantes sources d'investissement direct étranger peuvent donc être perdants. Enfin, il y a des effets sur les termes de l'échange, examinés plus haut.

- Les études centrées sur les mouvements des personnes qui fournissent des services montrent qu'il s'agit d'un moyen de livraison essentiel pour les pays exportateurs. Comme c'est le cas avec les études analysant la mobilité des facteurs et l'IDE, il semble que la libéralisation concomitante supposée des marchés de facteurs soit la principale source de gains importants. Cependant, les travaux sur le mode 4 ne vont pas sans difficultés, en particulier parce que la plupart des pays exigent que les travailleurs étrangers soient payés au même tarif que les ressortissants nationaux - ce qui atténue l'avantage des fournisseurs des pays en développement sur le plan des coûts. $\mathrm{Ne}$ pas reconnaître ce fait peut limiter la pertinence des études quantitatives faisant état de gains attribuables à la mobilité accrue de la main-d'oeuvre. De même, les études ne tiennent pas toujours suffisamment compte des cadres réglementaires existants qui régissent les marchés du travail, notamment en ce qui concerne la rémunération, les conditions de travail et la reconnaissance des qualifications.

- Les études économétriques analysant les effets dynamiques de la libéralisation et son incidence sur des secteurs particuliers aboutissent à des gains plus importants que les simulations des modèles d'équilibre général calculable (EGC). Ce résultat est sans doute imputable à l'élément dynamique des études économétriques qui tiennent compte des ajustements à long terme s'effectuant à travers l'accumulation de capital, la croissance démographique et le progrès technologique. 
234. D'un point de vue méthodologique, l'étude de l'OCDE montre qu'il est important d'envisager la libéralisation des échanges de services dans un contexte général, plus large en tout état de cause que celui de la libéralisation des échanges des marchandises.

- Premièrement, la libéralisation des services ouvrira des marchés à de nouveaux fournisseurs locaux, aussi bien qu'étrangers, et aura comme conséquence notable d'entraîner des gains qui découleront non pas de la recherche d'un meilleur accès aux marchés des services étrangers, mais du renforcement de la concurrence et de l'efficience sur le plan local.

- Deuxièmement, il est essentiel, pour connaître les effets de la libéralisation des services, de déterminer si un marché véritablement concurrentiel a été créé. La transformation de monopoles publics en monopoles privés engendre des avantages négligeables et, de même, la structure de la concurrence dans l'industrie influe considérablement sur la répartition des avantages de la libéralisation. Les travaux que mène actuellement l'OCDE sur la libéralisation des échanges de services dans les pays d'Europe du Sud-Est montrent que ces pays ne retireraient vraisemblablement pas d'avantages à ouvrir leurs services de télécommunications sur réseau fixe à des capitaux étrangers sans faire disparaître dans le même temps les restrictions qui s'appliquent actuellement aux nouvelles entrées.

- $\quad$ Enfin, certaines études sectorielles montrent que l'ordre de succession des réformes (par exemple, la privatisation, puis l'introduction de la concurrence) peut aussi être une considération importante.

235. Une réserve s'impose. L'évaluation des obstacles aux échanges de services et la quantification des avantages de la libéralisation dans ce secteur constituent un champ d'étude assez nouveau, caractérisé par des cadres théoriques, des techniques de modélisation et des séries de données qui ont toujours besoin d'être complétées. Les résultats présentés dans cette partie sont par conséquent soumis aux limites méthodologiques et statistiques inhérentes à l'état actuel des connaissances. Certaines d'entre elles sont examinées plus en détail dans la section suivante. 


\section{PROBLÈMES DE MÉTHODOLOGIE ET D'INTERPRÉTATION}

\section{Caractéristiques distinctives des services : différences entre services et biens}

236. La plupart des discussions sur les avantages potentiels de la libéralisation du commerce des services partent de l'hypothèse qu'une plus grande ouverture de ces échanges est bénéfique aux pays selon un schéma analogue à celui de la libéralisation du commerce des marchandises. Ce raisonnement se fonde sur deux impressions : les divers pays ont des avantages comparatifs différents, mais ces derniers sont les mêmes pour la production de biens et pour les services, et une plus grande ouverture permettra de mieux exploiter les avantages comparatifs de tous les pays. Il est certes tentant de considérer que les théories portant sur les avantages du libre échange s'appliquent de façon uniforme aux biens et aux services, mais cette optique soulève un certain nombre de difficultés.

237. A supposer même que l'on puisse traiter les biens et les services comme analytiquement similaires, il existe des nuances quant aux avantages que procure aux pays en développement la libéralisation des échanges de services; ces nuances ont été exposées dans de nombreux documents sur la politique des échanges, dont certains avancent des arguments préconisant un tarif douanier optimal (amélioration des termes de l'échange par le biais de la protection), la protection des industries naissantes, des tarifs douaniers qui transfèrent les rentes (transferts de rentes) ou qui viennent compenser certaines distorsions intérieures.

238. De plus, les services comportent des caractéristiques qui ne permettent pas de se servir d'un raisonnement conçu à l'origine pour les échanges de marchandises et nécessitent un effort de modélisation tenant explicitement compte de certaines de leurs spécificités. Ainsi :

- De nombreux services exigent une proximité entre producteurs et consommateurs : les échanges des services ne se limitent donc pas à des livraisons transfrontières, mais suppose également des déplacements de la part des fournisseurs et des consommateurs.

- Certains obstacles aux échanges de services ne sont pas discriminatoires (ainsi, un examen des besoins économiques limitant le nombre total de pharmacies pour 1000 habitants peut restreindre l'entrée des entreprises locales comme des entreprises étrangères). La libéralisation des échanges de services, en même temps qu'elle permettra l'entrée d'éléments étrangers, ouvrira donc les marchés à de nouveaux fournisseurs locaux. Par conséquent, elle apportera des avantages considérables découlant de l'intensification de la concurrence et à de l'efficacité accrue de la production.

- Le terme de services recouvre un ensemble hétérogène d'activités : banque, assurance, transport, télécommunications, services de consultation, commerce de détail et de gros, etc. Une partie de ces activités joue un rôle important en facilitant les transactions et en fournissant la fonction économique d'intermédiation, soit dans le temps (banque, assurance), soit dans l'espace (télécommunications, transport, commerce de détail, commerce de gros), de sorte que, lorsqu'elles sont explicitement modélisés en tant que telles, elles peuvent avoir des incidences théoriques différentes du point de vue de la libéralisation des échanges, comme l'a fait remarqué Melvin (1989). 
- Pour les marchandises, ce sont en général les douanes aux frontières nationales et d'autres obstacles physiques au commerce qui entravent le flux des échanges. Dès lors, les discussions afférentes à la libéralisation des échanges de biens portent en majeure partie sur les droits de douane, et moins sur les autres instruments. Dans le cas des services, les mesures affectant les échanges sont plus nombreuses et plus diverses, et comprennent des actions qui concernent les prestataires nationaux tout autant qu'étrangers. La tendance est de réglementer strictement les services, afin, notamment, d'atteindre des objectifs politiques majeurs, et les réglementations ne correspondent pas nécessairement à des obstacles aux échanges. En outre, pour certains secteurs de services, comme la banque, l'assurance et les télécommunications, les échanges transfrontières sont faibles. En effet, la présence commerciale joue un rôle important pour la fourniture de ces services, mais elle n'est pas prise en compte dans les statistiques usuelles sur les échanges. Dans les cas ou une présence commerciale est nécessaire, la production totale ou la consommation totale sont plus proches du concept quantitatif correspondant que le total des échanges transfrontières.

239. Dès lors, pour analyser la libéralisation des échanges de services et son impact sur les pays en développement, il importe de spécifier clairement et exhaustivement les types et les formes de libéralisation en cause. Par conséquent, ces derniers doivent souvent être étudiés selon des critères qui n'interviennent pas dans la libéralisation du commerce des marchandises.

\section{Importance de l'accès au marché et du traitement national : renforcement de la concurrence intérieure et amélioration de l'efficacité de la production}

240. Les restrictions des échanges n'ont pas les mêmes caractéristiques que pour les marchandises aux frontières ; leur variété est plus grande et elles s'appliquent aussi sur les marchés intérieurs et non pas seulement aux frontières. L'AGCS mentionne les obstacles à l'accès au marché qui protègent les entreprises en place contre de nouveaux concurrents locaux aussi bien qu'étrangers. Il suppose ainsi que la libéralisation des échanges de services ouvrira les marchés des services des pays en développement à de nouvelles entreprises locales autant qu'étrangères.

241. Outre les avantages que procurent l'accès aux marchés extérieurs et l'exploitation des avantages comparatifs, on peut attendre d'importants gains de bien-être des évolutions suivantes :

- renforcement de la concurrence intérieure résultant de la libéralisation de l'économie nationale ;

- réduction des coûts intérieurs et diminution des possibilités de rentes au bénéfice des producteurs en place, grâce à des réformes accentuant la concurrence et à l'abolition de règlements tatillons générateurs d'inefficacités.

242. En raison des changements sous-jacents à la structure du marché, la croissance provenant du commerce des services se trouvera vraisemblablement accentuée également dans le cas où le pays qui procède à la libéralisation se trouve comparativement désavantagé dans le secteur concerné. Cet argument est explicité par Mattoo et al. (2001) ; Francois et Wooton (2000) le sous-entendent également lorsqu'ils mettent l'accent sur les liens entre l'intensité de la concurrence, le type et le degré d'accès au marché et les effets d'incitation à la concurrence que fournit la libéralisation. Cet aspect des services doit absolument être pris en considération dans toute évaluation des effets de la libéralisation. Tant la communauté des fournisseurs de services que les publications relatives à la politique générale s'accordent à dire que les résultats d'une libéralisation des services seront fortement tributaires du contexte réglementaire et de la perception de la nécessité d'inscrire la libéralisation dans un cadre réglementaire adéquat. 


\section{Le problème des services ayant un rôle d'intermédiaires}

243. Comme indiqué plus haut, certains services ne donnent pas directement un résultat final, mais servent uniquement à faciliter les transactions, en remplissant une fonction économique d'intermédiation, soit en temps, soit en espace. Les économistes appellent «marges » de telles activités. Comme le relève Melvin (1989), les services, s'ils sont explicitement modélisés en tant que tels, peuvent avoir des conséquences différentes pour la libéralisation du commerce. C'est ainsi que Ryan $(1990,1992)$ démontre que, lorsque la fonction bancaire est modélisée explicitement comme service d'intermédiation ne fournissant pas en soi un résultat mais servant à faciliter les relations entre emprunteurs et prêteurs, la libéralisation des échanges de services bancaires peut faire baisser le PIB, et même le niveau de vie. Chia et Whalley (1997) ont fourni un exemple chiffré de libéralisation de services bancaires responsable d'une diminution du bien-être sur la base de cette approche. Les résultats de tels exemples proviennent d'une utilisation de formulations spécifiques, de valeurs paramétriques déterminées et de formes fonctionnelles particulières : ils n'ont donc pas un caractère général, mais ils pourraient néanmoins jeter le doute sur l'hypothèse généralement retenue selon laquelle les pays développés et les pays en développement plus pauvres se partageraient automatiquement les bénéfices d'une libéralisation du commerce des services à l'échelle mondiale. Bhatterai et Whalley (1999) procèdent à une analyse similaire des implications de la libéralisation des services de réseaux (c'est-à-dire les télécommunications) : on y retrouve l'idée que la prise en compte des caractéristiques particulières de services déterminés modifie l'analyse des impacts de la libéralisation des services.

244. Selon l'expérience acquise en Australie en matière de modélisation des activités de «marges », il ne fait guère de différence, en pratique, selon que l'on traite les services comme des « marges » ou comme des intrants intermédiaires traditionnels (qui, eux non plus, ne fournissent pas directement de résultat final). S'il est vrai que le résultat sera différent selon que l'on traite ces activités comme des intermédiaires ou comme des produits finaux, les études EGC considèrent systématiquement les services comme des intermédiaires. Il est possible, théoriquement que lorsque des services d'intermédiation sont représentés sous leur forme économique réelle, plutôt qu'ad valorem, les deux théorèmes fondamentaux de l'économie du bien-être ne se vérifient plus. Il a cependant été démontré à suffisance que cela ne constitue pas un problème en pratique. Ces démonstrations proviennent de plusieurs décennies de modélisation EGC de l'économie australienne depuis le modèle ORANI (Dixon, Parmenter, Sutton et Vincent). Dans ce cadre, on a modélisé explicitement jusqu'à huit «services marges » majeurs (commerce de gros, commerce de détail, transport - aérien, routier, ferroviaire et maritime - assurance, hôtels et restaurants) en tant qu'activités de marges non-ad valorem, plutôt qu'en tant qu'apports intermédiaires traditionnels, sur la base de données fournies par le Bureau australien des statistiques. Dans les nombreuses applications universitaires et gouvernementales exécutées, le traitement des marges n'a jamais rien donné d'autre que des résultats conventionnels.

\section{Identification et mesure des obstacles et des réglementations concernant les services}

245. La manière dont les règlements et les obstacles s'appliquent aux services posant un certain nombre de problèmes conceptuels, plusieurs réserves et mises en garde s'imposent si l'on veut évaluer avec précision l'efficacité des différentes restrictions.

- Les échanges de services sont touchés par une plus grande diversité d'obstacles que les échanges de biens : il ne s'agit pas uniquement de mesures discriminatoires à l'encontre des entreprises étrangère : ces obstacles peuvent aussi empêcher l'entrée sur le marché d'entreprises nationales. Par conséquent, les effets de la réglementation qui s'applique aux services dépassent les frontières puisque celle-ci soulève des problèmes de contestabilité du marché local tant en ce qui a trait aux mesures régissant l'accès au marché (qui restreignent la contestabilité des marchés) qu'aux politiques relatives au traitement national (qui établissent une distinction entre les 
fournisseurs locaux et étrangers au bénéfice des fournisseurs locaux). Il se peut que ces obstacles à l'accès au marché réduisent l'offre locale de même que l'offre étrangère, les approches axées sur l'évaluation quantitative de cette dernière ne tenant peut-être pas compte des effets de la première.

- Étant donné que les réglementations applicables aux services visent généralement à répondre à divers objectifs d'action, il convient, dans le cadre du débat sur la libéralisation des services, d'évaluer le poids global de la réglementation et d'identifier, si possible, des mesures tout aussi efficaces mais ayant un effet moins restrictif sur les échanges. Les comparaisons quantitatives de ces moyens d'action sont ardues et nécessitent l'élaboration de méthodes de mesure perfectionnées [voir Appendice 1].

\section{Identification et mesure des obstacles}

246. Les obstacles à la libre circulation des services à l'échelle internationale sont beaucoup plus divers que dans le cas des marchandises et il est donc nécessaire de disposer d'autres moyens de les formaliser et de les conceptualiser. La nature des restrictions au commerce de services est déterminée par les caractéristiques propres à ces derniers : leur nature intangible, l'encadrement réglementaire très dense visant à éviter toute défaillance des marchés et à atteindre des objectifs sociaux non économiques, la nécessaire proximité des fournisseurs de services et des consommateurs, et la mobilité des facteurs associée à leur commerce. Nombre de ces restrictions revêtent une forme réglementaire et s'inscriraient peut-être mieux dans la vaste documentation sur l'organisation industrielle que dans des documents ordinaires sur les échanges. C'est ainsi, par exemple, qu'il peut exister des obstacles à l'accès de fournisseurs de services étrangers aux marchés nationaux. Ces obstacles, à classer dans la catégorie du mode 3 de l'AGCS, peuvent néanmoins concerner non seulement le droit d'établissement, mais également des règles d'accès et des codes de conduite spécifiques au secteur. Chacun de ces ensembles de règles est propre à un domaine d'activité déterminé et fonctionne différemment d'un secteur à l'autre.

247. En gros, deux approches différentes ont été utilisées dans le cadre des travaux qui ont été menés pour classer les obstacles aux échanges de services afin d'en mesurer la taille et de soutenir le débat sur leur libéralisation plus poussée. La première, typique des premiers travaux, et susceptible de donner des résultats trompeurs, consistait à traiter tous les services comme une entité homogène. La deuxième, retenue dans les travaux plus récents, examine séparément la structure de la réglementation, les obstacles à l'entrée et les restrictions affectant la mobilité dans chaque marché de services. Les restrictions/réglementations diffèrent selon qu'elles s'appliquent, par exemple, aux banques et aux transports routiers, en raison des caractéristiques différentes de chaque type de service. Le fait de restreindre la valeur des réserves qu'une banque étrangère peut maintenir dans un pays n'a pas tellement à voir avec celui d'exiger que des camions passent une frontière avec un réservoir plein. Pour comparer l'impact économique de ces restrictions, on peut par exemple examiner leurs implications en termes de coûts. Toutefois, certaines restrictions supposent l'engagement de frais fixes pour entrer sur un marché, d'autres modifient le coût marginal, et d'autres établissent concrètement une quantité maximale de services fournis, ou définissent des normes de qualité minimum. Comparer les obstacles et évaluer leurs impacts est donc par définition une tâche difficile, et l'hétérogénéité qui caractérise les grands secteurs de services (secteur bancaire et secteur des transports, par exemple) rend les choses encore plus complexes, de même que celle qui existe à l'intérieur des différentes catégories (par exemple entre les types de services offerts par les institutions financières).

248. Il est pourtant possible de tenter de procéder à des évaluations sur la base des quatre modes de fourniture de services définis par l'AGCS, qui reconnaît que les restrictions à ces transactions revêtent souvent la forme d'obstacles non tarifaires et sont mises en place pour limiter non seulement l'entrée de services étrangers, mais aussi l'accès de fournisseurs et/ou consommateurs nationaux et étrangers en général au marché intérieur. 
249. La multiplicité des pratiques restrictives en matière d'échanges rend difficile l'identification des contraintes. Premièrement, alors que les données sur la fréquence ne font qu'évaluer si les restrictions s'appliquent à chacun des 620 secteurs couverts par l'AGCS pour un type de service donné et à chacun des quatre modes de fourniture, il n'est pas certain que l'ensemble des restrictions constituent, dans la pratique, des contraintes aux échanges. Certaines pratiques peuvent être restrictives et d'autres non. Certaines pratiques peuvent avoir un effet amplifié et d'autres peuvent s'annuler. Deuxièmement, les restrictions n'ont pas dans tous les cas le même impact quantitatif. Leurs effets marginaux sur les échanges sont en général différents. Enfin, les mesures des restrictions peuvent masquer la discrimination exercée par les pays dans l'application des obstacles, même si la discrimination de jure et la discrimination de facto sont toutes deux considérées comme des infractions à la clause du traitement national de l'AGCS. Les subventions et les restrictions font parfois l'objet d'une compensation dont il faut tenir compte. Il arrive également que l'application de facto de la réglementation diffère de son application de jure, tout comme les droits appliqués diffèrent des droits consolidés dans le cas des échanges de marchandises.

250. Pour mettre en évidence les contraintes, les études économétriques font de plus en entrer séparément différents composants du cadre réglementaire dans les régressions. Dans les travaux récents, on a cherché à distinguer les différentes façons dont les services peuvent être fournis. Un traitement typique consiste à modéliser les flux d'IDE comme étant liés à la prestation de services suivant le mode 3 de fourniture de l'AGCS (présence commerciale à l'étranger). Les dispositions sur la mobilité de la main-d'œuvre en vertu du mode 4 de fourniture de l'AGCS (mouvement temporaire des fournisseurs de services) ont également été modélisées séparément. La désagrégation des données sur les obstacles aux échanges de services demeure une priorité de premier plan des recherches à venir.

251. Une évaluation détaillée des obstacles et de la réglementation devrait permettre de déterminer :

- $\quad$ si la réglementation constitue réellement un obstacle ;

- $\quad$ si une réglementation donnée, visant à atteindre un objectif donné, mais influant défavorablement sur les échanges de services est inutilement pesante ;

- $\quad$ si d'autres mesures tout aussi efficaces mais ayant un effet moins restrictif sur les échanges sont envisageables.

252. En résumé, les progrès enregistrés dans la compréhension des services et des échanges de services se reflètent également dans l'évaluation des obstacles et des restrictions qui les touchent. Des méthodes de plus en plus perfectionnées montrent que les obstacles posés à la prestation des services sont complexes, que leurs effets sont nombreux et qu'ils peuvent concerner l'entrée sur des marchés locaux (droits d'établissement, ou droit de fournir des services), les règles de conduite (réglementation), le nombre et la taille des concurrents sur un marché (réglementation relative à la concurrence), et d'autres aspects. Là encore, la structure du marché, le comportement et les résultats sont des éléments clés et doivent être évalués dans le cadre de l'examen des impacts quantitatifs de la libéralisation des échanges internationaux de services dans les pays en développement.

\section{Impact de la libéralisation sur l'ensemble de l'économie}

253. Il existe deux méthodes quantitatives principales pour évaluer l'impact économique de la libéralisation des services. La première fait appel à des modèles calibrés (ou calculables) du commerce international, essentiellement d'équilibre général, et s'efforce de quantifier les effets de la libéralisation des services sur le bien-être mondial et sur les économies des différents pays, grâce à une approche structurelle 
visant à montrer comment les entraves à un secteur se répercutent sur d'autres et sur l'économie tout entière. La seconde recourt à des techniques économétriques pour étudier l'impact d'une libéralisation spécifique à un secteur sur la croissance économique dans son ensemble, ou sur des mesures de la performance propre à un secteur (c'est-à-dire productivité, efficacité, coûts de production, etc.). Ces deux approches sont exposées aux Appendices 2 et 3.

\section{Interprétation des résultats de la modélisation EGC}

254. L'interprétation des résultats obtenus sur la base de publications exploitant des modèles existants pour quantifier les impacts de la libéralisation dans le domaine des services donne lieu à controverse, même s'il ne faut pas accorder d'importance excessive aux chiffres produits par les études EGC pour des pays déterminés : les résultats obtenus à partir de modèles calibrés devraient simplement être pris comme des compléments à la théorie, pour aider à comprendre quels types de forces peuvent être importants et comment différents facteurs peuvent interagir.

255. Le calibrage fait intervenir des opérations de simulation numérique utilisant des modèles d'équilibre général (souvent à l'échelle mondiale) qui reposent sur des modèles conventionnels de libéralisation du commerce de marchandises (voir Whalley (1985). Pour ces opérations, les services de production sont généralement représentés sous forme d'un intrant destiné à une production intermédiaire; les obstacles au commerce de services se présentent sous forme de restrictions de type droits ad valorem, de taxes ou d'incidents de productivité affectant le rendement, de facteurs ou de frais fixes. Les résultats sont alors déterminés par l'importance des obstacles initiaux, la manière dont ils évoluent dans le cadre d'une libéralisation, leur élasticité, l'importance des échanges de services ainsi que par la taille relative du pays en cause et les différences éventuelles de structure du marché.

\section{Résultats contradictoires}

256. Un des problèmes avec l'interprétation des résultats des études disponibles est qu'à première vue, les résultats peuvent paraître contradictoires et semer le doute, en particulier pour certains pays en développement pris individuellement. Par exemple, Dee et Hanslow (2000) ont publié des résultats faisant état des bénéfices considérables tirés par certains pays en développement de la libéralisation des services dans le cadre du cycle d'Uruguay (progression de $14.6 \%$ du PIB dans de cas de la Chine et de $5.1 \%$ pour l'Indonésie). Ils indiquent également que, d'une manière générale, plus de la moitié du total des avantages provenant de la libéralisation des biens et des services sont dus à la libéralisation de ces derniers. Par contre, en utilisant des données GTAP similaires, Robinson et al (1999) arrivent pour la Chine à une progression de $0.34 \%$ du PIB, de $1.29 \%$ pour les pays de l'ANASE, et de $1.13 \%$ pour l'Asie du Sud. Verikios et Zhang (2001) indiquent que la libéralisation des télécommunications a entraîné des pertes pour la Malaisie et qu'il en a été de même pour l'Indonésie à la suite de la libéralisation des services financiers. Ils n'affichent que des bénéfices modestes dans le cas de la Chine.

257. On peut cependant relever d'importantes différences d'approche entre ces études, ce qui montre qu'il n'existe encore aucune convergence, à ce stade précoce des travaux de modélisation des échanges de services, quant à la méthode et à l'ensemble d'obstacles initiaux à privilégier ; en outre, les auteurs n'ont pas tous la même maîtrise des modalités de modélisation des services et de prise en compte des éléments critiques. C'est ainsi que Dee et Hanslow incorporent explicitement les restrictions du mode 3 de l'AGCS, alors que l'étude antérieure de Robinson et al. utilise une approche de modélisation qui ne fait pas la distinction entre les flux de facteurs entre pays et la fourniture de biens et de services. De plus, les deux modèles comportent des différences d'évaluation des obstacles. Brown, Deardorff et Stern estiment que les avantages résultant du cycle de Doha représenteront 574 milliards d'USD par an à l'échelle mondiale, la plus grande partie (413 milliards d'USD) provenant des services, et que les bénéfices les plus importants, en termes absolus, iront aux pays développés. Verikios et Zhang, tenant compte des critiques faites 
habituellement aux modèles calibrés, séparent le traitement des services financiers et des télécommunications, ce qui leur permet de prendre en considération des éléments spécifiques à chacun de ces services. Ils appliquent aussi des estimations distinctes pour les obstacles rencontrés dans chacun de ces secteurs. Ils ne prennent pas pour hypothèse (contrairement à Dee et Hanslow) que ces obstacles sont représentatifs des obstacles rencontrés dans d'autres secteurs de services. Enfin, leur estimation de la mobilité des capitaux est inférieure aux chiffres retenus par Dee et Hanslow.

258. On voit donc que les écarts dans les estimations sur les avantages tirés de la libéralisation du commerce de services proviennent surtout de divergences relatives à l'importance des obstacles initiaux et reflètent l'absence d'accord sur un ensemble d'estimations à privilégier. Il faut par conséquent analyser et comparer les résultats des diverses études en tenant compte de l'importance que chacune de ces études attache aux obstacles initiaux. Ce simple fait explique une grande partie des contradictions apparentes.

\section{Types de modèles : prise en compte des modes 3 et 4}

259. Les modèles EGC prédisent tous que la libéralisation du commerce et/ou des investissements afférents aux services fera augmenter les revenus réels à l'échelle de la planète. Par contre, la répartition entre pays des profits de la libéralisation tend à dépendre fortement des spécifications de chacun de ces modèles. Dans ceux qui ne modélisent que le mode 1 (transfrontières) de fourniture, sans traitement endogène de l'IDE, les bénéfices de la libéralisation des échanges de services tendent à être positifs dans le monde entier. Certains modèles plus récents, cependant, prennent en compte les firmes multinationales qui décident, pour des raisons qui leur sont propres, soit d'exporter, soit de créer une succursale dans le pays visé. D'autres modèles, comme celui de Markusen, Rutherford et Tarr (2000) considèrent les fournisseurs étrangers de services de production comme un bien intermédiaire.

260. Les études qui traitent explicitement l'investissement direct étranger comme un mode de fourniture de services parviennent à des conclusions très différentes. Dee et Hanslow (2000) trouvent que la libéralisation du commerce de services fait augmenter les revenus mondiaux dans leur ensemble, tout en admettant que certains pays enregistrent de faibles baisses de leur bien-être. Leur modèle prévoit une libéralisation des restrictions touchant à l'IDE ainsi qu'au commerce. Les origines de la baisse du niveau de vie peuvent être de trois ordres. Tout d'abord, la libéralisation peut inciter certains investisseurs à se tourner vers des pays où les obstacles aux investissements étrangers étaient auparavant relativement élevés. En deuxième lieu, les obstacles peuvent générer des rentes, dont certaines bénéficient aux détenteurs de capitaux étrangers. Avec la libéralisation, ces rentes se trouvent érodées par la concurrence et les pays qui constituent d'importantes sources d'IDE peuvent y perdre. Restent enfin les effets liés aux termes de l'échange, évoqués plus hauts. Par exemple, Dee et Hanslow attribuent essentiellement à des diminutions de rentes les pertes subies par les États-Unis, cependant que celles frappant le Canada sont dues avant tout aux effets des termes de l'échange. Brown et Stern (2001), s'efforçant d'améliorer leur précédente étude (Brown et al., 1996), incorporent un traitement explicite de l'IDE, ainsi que certains éléments de la structure de production, s'inspirant ainsi de Markusen, Rutherford et Tarr (2000). Ils obtiennent des prévisions relativement larges concernant les effets de la libéralisation des échanges de services sur le bien-être, qui seraient là aussi globalement positifs à l'échelle mondiale, mais négatifs pour certains pays. Ils rendent compte de six simulations distinctes avec, pour chacune d'elles des hypothèse différentes en matière de mobilité des capitaux ou de demande. Brown et Stern font remarquer que, dans leur modèle, les baisses de bien-être tendent à être associées à des sorties de capitaux suscitées par des perspectives d'investissement plus prometteuses dans d'autres parties du monde. Ils postulent que, dans le cadre de scénarios selon lesquels tous les pays libéraliseraient, à l'exception d'un seul, les pertes qu'enregistrerait ce dernier seraient plus fortes que dans tout autre scénario. Ce point fait écho à un argument de la théorie économique qui veut que si le reste du monde libéralise, les prix mondiaux évoluent et un pays isolé peut se retrouver perdant s'il choisit le statu quo à l'intérieur de ses frontières. 
261. Le mode 4 (mouvement temporaire de personnes en relation avec la fourniture de services), essentiel à de nombreuses exportations de services, revêt une importance cruciale pour les pays en développement où la main-d'œuvre et/ou le capital humain abondent. En modélisant explicitement ce mode 4, la libéralisation de la fourniture de services semblerait une source possible d'impacts plus marqués. Néanmoins, les travaux portant sur le mode 4 présente certaines difficultés, notamment du fait que la plupart des pays exigent que les travailleurs étrangers soient rétribués selon les mêmes barèmes que les nationaux (ce qui réduit les avantages dont pourraient bénéficier les fournisseurs de pays en développement sur le plan des coûts). En ne tenant pas compte de ce facteur, on limite la portée des études quantitatives montrant les avantages d'une plus grande mobilité de la main-d'œuvre. De même, les études n'accordent pas toujours l'attention nécessaire aux cadres réglementaires existants sur le marché du travail, notamment en ce qui concerne les salaires, les conditions de travail et la prise en compte des qualifications. En fait, on pourrait même soutenir l'argument selon lequel l'avantage que représentent actuellement les coûts salariaux moindres dans les pays en développement se vérifie davantage, pour les échanges des services, dans le mode 1 que dans le mode 4. Dans certains secteurs, le recours à des services extérieurs (fourniture transfrontières du mode 1) est dû, notamment pour les services professionnels et informatiques, aux salaires plus bas et à la grande qualité des professionnels de pays en développement. Ce type de sous-traitance, qui ne se limite plus à la saisie de données mais porte de plus en plus sur des travaux de recherches et autres fonctions spécialisées, fait appel à la fourniture de services au titre du mode 1 ; cette réalité n'est peut-être pas adéquatement prise en compte dans les études disponibles actuellement.

262. En conclusion, les études qui modélisent explicitement les modes de fourniture 3 et 4 indiquent que, si les services sont libéralisés sans que cela les marchés de facteurs le soient également, comme dans le cas des marchandises, les bénéfices pour le commerce sont modestes. Si, par contre, la libéralisation des services se transforme en mécanisme éliminant les entraves aux flux de facteurs (IDE), les bénéfices sont importants et ne sont pas répartis de façon identique entre les pays. Il est utile de relever que d'après ces modèles, les effets les plus marquants ne proviennent pas de la libéralisation des échanges de services en tant que telle, mais de la libéralisation concomitante des marchés de facteurs qu'elle sous-entend. On retrouve en cela ce que l'on avait constaté pour le commerce des biens ; la libéralisation des marchés des facteurs accompagnant celle des échanges de marchandises à l'échelle internationale a des effets parfois prononcés mais inégalement répartis selon les pays.

263. L'une des fonctions majeures de la libéralisation du commerce des services pourrait donc consister à faciliter la libéralisation des marchés de facteurs à l'échelon mondial, en facilitant les flux de facteurs au titre des modes 3 et 4 de l'AGCS. Une telle libéralisation pourrait être difficile à atteindre directement; si l'on y parvenait indirectement dans le cadre d'une libéralisation des échanges de services, on pourrait peut-être en retirer des bénéfices considérables, notamment pour les pays en développement. En attirant davantage de capitaux et en dégageant de la main-d'œuvre pour mieux répartir les ratios de facteurs entre de grands blocs de pays, on peut sans doute obtenir le gain le plus important à l'échelle mondiale et la meilleure répartition entre les pays.

\section{Interprétation des résultats des études économétriques}

264. Si leur portée est plus limitée que celle des modèles EGC, les études économétriques n'en constituent pas moins un complément important aux modèles calibrés. Il est possible de recourir à des essais statistiques et à des théories confirmées pour évaluer la fiabilité et l'exactitude des prédictions qui en découlent, par ailleurs, l'apport de données nécessaire étant moindre, l'impact d'informations de piètre qualité s'en trouve réduit. Tout en ayant apparemment une portée politique considérable, les études économétriques posent quelques problèmes en matière d'interprétation des résultats : en effet, il est indispensable de disposer de variables de contrôle appropriées (pour tous les autres facteurs influant sur les performances), ainsi que de moyens permettant de traiter, lorsque cela est nécessaire, des problèmes de simultanéité. [Voir Appendice 3 pour un exposé plus détaillé de cette question] 
265. Au premier abord, les études économétriques visant à déterminer les effets de la libéralisation sur la croissance à l'échelle de l'économie semblent montrer sans ambiguïté que la libéralisation des secteurs de services ouvre la voie à une croissance et à un développement plus rapides, suggérant que la levée des obstacles aux échanges de services pourrait également permettre aux pays en développement d'accélérer leur croissance. Parmi les études économétriques récentes qui associent croissance et libéralisation du commerce de services, on citera celle de Francois et Schuknecht (2000), et celle de Mattoo, Rathindran et Subramanian (2001). Il importe par ailleurs de souligner que Mattoo et al. constatent des taux de croissance pouvant atteindre jusqu'à $1.5 \%$ de plus dans les économies qui ont fait le choix de libéraliser leurs télécommunications et leurs services bancaires; selon Francois et Schuknecht, l'expérience montre également que les mesures de libéralisation des services ont des effets considérables sur la croissance.

- Toutefois, l'interprétation des résultats présente certains problèmes : les études réalisées sont critiquées par Rodriguez et Rodrik (1999) qui estiment que ce sont les variables exclues qui pourraient entraîner la croissance la plus importante. Par exemple, une croissance plus forte peut résulter en grande partie d'une hausse des taux d'épargne et d'investissement, créant un recours accru à l'intermédiation, et donc aux services. L'amélioration de la croissance pourrait donc résulter davantage du renforcement des investissements que de l'utilisation plus intensive des services suite à leur libéralisation.

- Le fondement théorique de ces travaux économétriques présente des faiblesses et ne fournit pas des données analytiques suffisantes pour tirer des conclusions satisfaisantes sur la libéralisation des échanges de services et sur ses répercussions en termes de croissance. Bien que Francois et Schuknecht soulignent le rôle des économies d'échelle et des structures de coûts dans les services financiers, ainsi que leurs liens avec la structure du marché, leur argumentation théorique (comme c'est le cas dans la plupart des travaux théoriques sur la libéralisation des échanges) met en évidence des effets de niveau isolés plutôt que des effets permanents sur la croissance. Il reste donc à déterminer si le gain de croissance découlant de la libéralisation n'est qu'une conséquence temporaire ou un résultat plus permanent.

\section{Conclusion et travaux à venir}

266. On peut tirer deux conclusions des travaux récents d'évaluation des avantages de la libéralisation des échanges de services. Premièrement, les prévisions concernant des pays particuliers sont très sensibles à la structure de modélisation; sans doute devons-nous chercher à mieux comprendre les forces qui régissent les échanges et l'investissement, ainsi que la manière dont les réglementations du secteur des services peuvent faire obstacle aux échanges. En ce qui concerne les modèles EGC, leur application au secteur des services est relativement récente ; ils sont utiles dans le cadre des travaux de recherche, mais doivent être maniés avec précaution dès lors qu'il est question de s'y référer pour définir des orientations politiques. La deuxième conclusion est la suivante : les modèles montrent bien la difficulté d'écarter, comme s'il s'agissait d'éléments d'importance mineure, certaines des difficultés ou des ambiguïtés qui ressortent d'un traitement théorique de la libéralisation des échanges de services. L'ordre de mise en œuvre des mesures de libéralisation et leur interaction avec le commerce de biens déjà en place pourraient avoir une influence déterminante sur le plan du bien-être. 
267. Toutefois, la théorie comme les techniques empiriques ne cessent de s'affiner, et l'on s'efforce également d'améliorer les données statistiques. Les nouvelles études de modélisation visent à donner un tableau plus fidèle des aspects économiques des secteurs de services et des échanges dans ce domaine, notamment pour ce qui concerne les différents modes de fourniture, leurs fonctions et caractéristiques distinctives, et une approche plus désagrégée des secteurs de services. D'un point de vue empirique, on introduit progressivement des estimations cohérentes des chiffres de l'IDE et des données FATS relatives aux activités des filiales étrangères aux niveaux bilatéral et sectoriel, afin de tenir compte de la présence commerciale, qui représente une part importante des échanges de services. Par ailleurs, les milieux universitaires et les organisations internationales comme le FMI, Eurostat, l'OCDE et l'OMC s'emploient depuis quelques années à accroître la fiabilité globale des données sur le commerce des services et à résoudre certains problèmes bien connus: insuffisance des données, absence de concordance et de compatibilité entre les séries de données existantes, désagrégation sectorielle insuffisante et absence de classification par mode de fourniture.

268. Il serait néanmoins intéressant de procéder à une analyse plus approfondie de certains aspects :

- Base théorique - Aussi bien en ce qui concerne les études économétriques que les modèles calibrés, il faut consolider les assises théoriques de la libéralisation des échanges de services et de ses liens avec la croissance.

- Désagrégation sectorielle - elle permettrait de mettre en évidence les éventuelles différences entre les secteurs afin de déterminer le type de libéralisation à entreprendre et d'aider à identifier les secteurs qui devraient être libéralisés en priorité.

- Distorsion du choix du mode de fourniture - Il faudrait s'attacher plus attentivement à déterminer dans quelle mesure les restrictions existantes incitent à fournir des services suivant certains modes plutôt que d'autres (par exemple le choix de la consommation à l'étranger mode 2 - plutôt que de la présence commerciale à l'étranger - mode 3 - dans le secteur des services d'enseignement) et l'impact de ces choix sur l'économie et la société.

- Échanges entre pays en développement - Les avantages potentiels pour les pays en développement de la libéralisation des marchés dans d'autres pays en développement sont un aspect important et insuffisamment traité dans le cadre des discussions sur la libéralisation des services $^{24}$.

- Suppression des obstacles qui induisent la création de rentes et font monter les coûts. On ne sait pas bien dans quelle mesure les avantages varient suivant que les obstacles génèrent des rentes ou font monter les coûts.

- Mesures relatives à l'accès aux marchés et au traitement national. Il serait très intéressant, d'un point de vue pratique, de savoir si les principaux avantages de la libéralisation découlent de la suppression des mesures limitant l'accès aux marchés ou de celles qui concernent le traitement

Sur le plan pratique, les résultats de la libéralisation des échanges entre pays en développement devraient faire l'objet d'analyses. D'un point de vue théorique, les travaux relatifs aux accords commerciaux régionaux font état d'un certain nombre de conséquences importantes en matière de répartition, d'accumulation et de localisation. [Voir Baldwin et Venables (1995) qui font le bilan de ces travaux dans Handbook of International Trade]. La baisse des prix devrait profiter aux consommateurs; en outre, les pays exportateurs et importateurs devraient bénéficier de l'exploitation des avantages comparatifs, ainsi que de l'amélioration des conditions d'accès aux marchés, pour les uns comme pour les autres. 
national, autrement dit, de déterminer comment l'arrivée de nouveaux fournisseurs nationaux intervient dans l'impact de la libéralisation.

- Effets sur la redistribution et les revenus à l'échelle internationale et nationale - Il faudrait déterminer dans quelle mesure l'efficience accrue influe sur le revenu national par le biais d'une baisse des prix des services produits sur le territoire national par des fournisseurs nationaux ou internationaux.

- Effets secondaires (négatifs) possibles, notamment : risque d'appauvrissement (rare, toutefois, dans le cas du commerce de marchandises), interactions possibles avec l'épargne et l'investissement au niveau national, effets négatifs éventuels sur les termes de l'échange. 


\section{Appendice 1. Comment mesurer les obstacles aux échanges de services}

L'évaluation des obstacles aux échanges de services s'appuie sur des travaux visant à mesurer les barrières non tarifaires imposées au commerce des marchandises. Les méthodes généralement employées pour mesurer la présence et l'ampleur de barrières non tarifaires s'inspirent de Deardoff et Stern (1998), qui classent les obstacles en fonction de leur fréquence et de leur impact sur les prix et les quantités. Il importe cependant d'introduire certaines adaptations afin de tenir compte des caractéristiques particulières du commerce des services. Dans ce contexte, elles seront réparties en deux catégories :

\section{Méthodes utilisées pour mesurer l'ampleur des restrictions sur les échanges de services.}

Ces méthodes convertissent des informations qualitatives sur les restrictions en données quantitatives comparables. Leur mise en œuvre comporte deux grandes étapes :

i. Collecte et classement des informations sur les restrictions, émanant de différentes sources.

ii. Élaboration et calcul d'un taux de restriction des échanges.

\section{i. $\quad$ Collecte des informations sur les restrictions}

Il est essentiel, avant de mesurer l'impact des entraves au commerce des services, de définir ces obstacles et d'en dresser la liste. L'AGCS a fourni une première liste des barrières qui freinent les échanges dans le secteur des services. Hoekman, par exemple, qui a fait œuvre de précurseur dans ses travaux sur le calcul de taux de restrictivité des obstacles aux échanges, a puisé des informations dans les listes d'engagements de l'AGCS afin de déterminer la fréquence des restrictions, pour l'ensemble des secteurs de services, dans les pays membres de l'OMC. De même, certaines mesures restrictives pesant sur le secteur des services financiers [Mattoo (1998), Classens et Glassner (1998)] ont été évaluées d'après ces mêmes listes d'engagements. Ces dernières ne tenant pas compte, cependant, de la totalité des barrières en place (*), elles ont été complétées dans les études ultérieures (et principalement celles menées sous les auspices de la Productivity Commission) par d'autres sources d'information sur la législation en vigueur en matière d'obstacles au commerce des services. Les rapports gouvernementaux, ceux des associations industrielles, et les documents établis par l'APEC (Organisation de coopération économique AsiePacifique), l'OCDE, l'OMC et les représentants des États-Unis pour le commerce extérieur, se sont révélés précieux pour le recensement des autres restrictions existantes.

\section{ii. $\quad$ Élaboration et calcul d'un taux de restriction des échanges}

A partir de ces informations qualitatives, on établit un taux afin de mesurer la sévérité des restrictions, en se fondant de préférence sur les modes de fourniture de services mentionnés par l'AGCS. On fixe, au moyen d'un système d'attribution de notes et de coefficients de pondération, un taux permettant de convertir les données qualitatives sur les restrictions en données quantitatives. Les notes sont attribuées en fonction du caractère plus ou moins contraignant des restrictions.

Comme indiqué précédemment, Hoekman (1995) a été le premier à instaurer un taux pour mesurer les limitations imposées au commerce des services. Il a mis au point, afin d'évaluer la nature et l'importance des engagements au titre de l'AGCS, une méthode de pondération comportant trois catégories. Aux fins de 
quantification, des notes -1 (régime très restrictif, aucun accès au marché), 0.5 (restrictions limitées à certains aspects) ou 0 (libre accès) - sont ainsi attribuées aux mesures restreignant l'accès aux marchés et recensées par l'AGCS, et les taux de couverture sont également évalués. Cependant, ce système ne prend pas en compte l'impact réel des différentes barrières sur l'économie, le même coefficient de pondération étant appliqué aux restrictions relativement mineures et aux mesures fermant quasi-complètement les marchés.

Les études menées récemment s'efforcent de surmonter certaines de ces faiblesses par le biais d'estimations fondées sur des bases de données qualitatives plus complètes et relatives aux mesures affectant les échanges de services, en appliquant des méthodes de pondération complexes pour évaluer le caractère restrictif des différentes mesures (cf. travaux réalisés conjointement par l'Australian Productivity Commission et l'Australian National University). Le classement et l'évaluation des coefficients de pondération tiennent compte des informations concernant différents types d'obstacles et l'impact économique probable de chacun d'eux. Enfin, s'agissant des restrictions examinées dans ces études, les auteurs font la distinction entre les restrictions relatives au traitement national et à l'accès aux marchés, mais aussi entre les restrictions portant sur l'établissement et celles qui concernent les activités en cours (c'est-à-dire après implantation).

Toutefois, ces méthodes ne permettent qu'une appréciation approximative du caractère restrictif des différents obstacles au commerce de services. Elles ne fournissent aucune information quant aux répercussions économiques de ces obstacles sur les prix, la production, la consommation et les échanges internationaux, ni sur les conséquences qui découleraient de leur maintien ou de leur suppression. Ces méthodes devraient donc, idéalement, être complétées par d'autres, à savoir :

\section{Méthodes utilisées pour mesurer l’impact des restrictions sur le commerce des services :}

En règle générale, trois méthodes sont possibles pour mesurer les effets des restrictions sur les prix et/ou les coûts des services :

i) conversion du taux de restrictivité des échanges en équivalent tarifaire ;

ii) méthodes indirectes consistant à déterminer un prix ou une quantité de référence et à imputer aux restrictions - en partie ou en totalité — tout décalage constaté par rapport à ces références ;

iii) méthodes directes consistant à faire une estimation des effets des restrictions — d'après le taux de restrictivité des échanges - sur la production, les prix et/ou les coûts des prestataires de services.

\section{i. $\quad$ Conversion du taux de restrictivité des échanges en équivalent tarifaire}

Ainsi que nous l'avons déjà mentionné, Hoekman (1995) utilise le taux de restrictivité comme point de départ pour calculer les équivalents tarifaires de l'importance relative des obstacles au commerce de services. Il effectue cette conversion au moyen d'un ensemble d'équivalents tarifaires de référence pour différents secteurs, allant d'une valeur de $200 \%$ pour les secteurs considérés comme très fermés (cabotage, transport aérien, services postaux, télécommunication vocale, assurance-vie) à des valeurs comprises entre 20 et $50 \%$ pour les secteurs dans lesquels l'accès au marché est moins limité. En multipliant les taux de couverture par les tarifs de référence, Hoekman a calculé les équivalents tarifaires par secteur et par pays. 


\section{ii. $\quad$ Méthodes indirectes}

Ces méthodes consistent à déterminer un prix ou une quantité de référence et à imputer aux restrictions - en partie ou en totalité — tout décalage constaté par rapport à ces références.

D'autres formules ont été appliquées pour quantifier l'impact économique des obstacles au commerce de services : elles tiennent compte de l'impact sur les prix (c'est-à-dire les répercussions des barrières non tarifaires sur les prix intérieurs, en comparant ces derniers aux prix mondiaux) et de l'impact sur la quantité de services fournis (comparaison du volume réel des échanges et du volume qu'ils pourraient atteindre).

S'agissant de l'impact sur les prix, les références sont calculées à partir de l'hypothèse selon laquelle un marché libre d'accès se caractériserait par des prix équivalents aux coûts marginaux. En présence de restrictions, en revanche, il y aurait un décalage entre le prix et le coût marginal. Par conséquent, les études empiriques fondées sur la mesure de l'impact financier ou de l'impact sur les prix dans les secteurs de services voient dans la marge prix-coût un indicateur de l'ampleur des obstacles aux échanges (voir Francois et Hoekman, 1999).

Des méthodes plus fiables sont aujourd'hui en cours d'élaboration. A partir de la constatation que les services fournis ne sont pas les mêmes, non seulement d'un prestataire à l'autre mais aussi, pour un même fournisseur, en fonction des consommateurs, les recherches ont montré l'impossibilité de présumer que le prix étranger est la référence la plus pertinente. Au lieu de cela, l'élément-témoin — à savoir : le prix qui serait pratiqué en l'absence d'obstacles au commerce — doit être établi à partir d'une modélisation économétrique des facteurs qui déterminent le prix intérieur pour chaque économie. Ces modèles sont généralement calculés au moyen de données relatives à plusieurs pays, mais c'est le prix intérieur (ou la différence prix-coût) qui constitue la variable dépendante dans chaque pays, et non l'écart prix intérieurprix étranger. Par ailleurs, les secteurs de services se caractérisent par une concurrence imparfaite, des produits différenciés et des coûts en hausse. Les marges prix-coûts seront positives, même sans barrières aux échanges. Le plus souvent, toutefois, elles seront plus faibles en l'absence d'obstacles. Les modèles économétriques s'appuient sur les variations entre pays pour évaluer l'importance de cette différence.

En ce qui concerne l'impact sur les quantités, il est possible d'évaluer - souvent de manière très globale - le volume des importations effectuées dans le cadre d'un régime imposant des restrictions, mais on ne dispose pas d'autres données quantitatives avec lesquelles établir une comparaison. Il faut pour cela un modèle fiable des déterminants commerciaux, ainsi que des données portant sur un éventail suffisamment large de cas de figure, de manière à pouvoir définir une situation qui permette au moins une certaine liberté dans les échanges. La méthode généralement utilisée pour mesurer les incidences des barrières non tarifaires sur les quantités fait appel à des modèles de régression applicables à différents produits ou à un ensemble de pays, qui permettent de calculer ce qu'auraient été les échanges en l'absence de barrières et de comparer les résultats aux chiffres réels. Ce type de méthode économétrique (modèles gravitationnels du commerce international) s'applique également au commerce de services. L'écart entre les importations réelles et les prévisions, considéré comme représentatif des obstacles, est converti en effetprix ou effet-coûts.

Les études les plus récentes (Warren, 2001) privilégient toutefois les quantités consommées ou produites. Deux raisons laissent à penser que ces quantités permettent une mesure plus exacte des performances que le volume des échanges. Premièrement, les obstacles aux échanges de services ne frappent pas exclusivement les prestataires étrangers : elles peuvent également empêcher l'entrée de services produits par de nouvelles entreprises nationales. Il se peut que ces entraves à l'accès au marché limitent la fourniture de services par des fournisseurs nationaux tout autant que les prestations transfrontières. Les méthodes axées essentiellement sur ce dernier aspect risquent alors de ne pas tenir compte du premier. Deuxièmement, dans certains secteurs de services, notamment les télécommunications, 
les échanges transfrontières sont peu développés. C'est par le biais de la présence commerciale que s'effectuent surtout les échanges; or, celle-ci ne figure tout simplement pas dans les statistiques commerciales traditionnelles. Lorsque la présence commerciale est nécessaire, la production ou la consommation totales sont plus proches du concept quantitatif correspondant que le total des échanges.

\section{iii. $\quad$ Méthodes directes}

Les méthodes les plus élaborées permettent d'obtenir directement une estimation de l'impact des restrictions sur les prix et/ou les coûts des prestataires de services. A partir de la théorie économique, on construit un modèle économétrique tenant compte des déterminants pertinents de la performance économique des entreprises, par exemple :

- les facteurs qui influent sur le secteur d'activité et sur l'économie dans son ensemble ;

- une mesure de la restrictivité des obstacles qui entravent les échanges (représentée par le taux de fréquence de ces obstacles, calculé précédemment).

La méthode économétrique est alors utilisée pour déterminer les incidences relatives des différents déterminants sur les prix et les coûts. Chaque fois que cela est possible, les composantes du taux de restriction des échanges (taux extérieur et taux intérieur) sont entrées séparément, afin que la modélisation économique révèle les effets des différents obstacles, ce qui améliore considérablement l'intérêt pratique des estimations.

Cette méthode, fondée sur les documents relatifs à l'organisation industrielle, peut également renseigner sur la mesure dans laquelle les obstacles aux échanges augmentent les marges prix-coût, générant ainsi des rentes économiques, ou accroissent les coûts au-delà de ce qui se serait passé en l'absence de restrictions :

- $\quad$ si les restrictions sont considérées comme créatrices de rentes, leurs effets sont modélisés sous la forme d'équivalents fiscaux (taxes sur la production, sur les facteurs de production ou sur les coûts fixes) ;

- $\quad$ si elles sont considérées comme la cause d'une augmentation des coûts, leurs effets sont modélisés sous la forme de modifications radicales de la productivité ayant un impact sur la production, les facteurs de production et les coûts fixes.

Les obstacles aux échanges de services pouvant entraîner ces deux effets en même temps, des recherches plus approfondies s'imposent pour mieux quantifier ces deux types d'incidences.

Note :

(*) Par exemple, McGuire (1998) a constaté que seules 38 des 165 mesures qui s'appliquent actuellement aux services financiers en Australie figuraient sur les listes établies dans le cadre de l'AGCS. Un certain nombre d'obstacles liés aux monopoles gouvernementaux sur la prestation de certaines catégories de services financiers, les réglementations prudentielles, les restrictions sur l'investissement étranger direct dans le secteur bancaire et les assurances, les exigences discriminatoires des autorités concernant les licences et les garanties gouvernementales relatives à la sélection des prestataires de services financiers n'apparaissent pas, du fait de l'approche des listes positives, dans les tableaux de l'AGCS. 


\section{Appendice 2. Modèles calibrés du commerce international}

Les modèles calibrés sont conçus pour traiter un large éventail de situations sur la base de données quantitatives. En théorie, un modèle détaillé d'équilibre général calculable (modèle EGC) mesurant les effets de la libéralisation des échanges de services doit tenir compte des différents modes de fourniture de services : mode 1 (échanges transfrontières), mode 3 (présence commerciale) et mode 4 (mouvements temporaires de personnes liés à la fourniture de services). La modélisation EGC relatifs à la libéralisation des services étant encore dans les premiers stades de son développement, les études menées jusqu'ici s'emploient essentiellement à rendre compte des principaux effets directs de la libéralisation. Toutefois, parmi les résultats de cette méthode susceptibles de contribuer à l'élaboration des politiques, figure une évaluation quantitative d'éléments essentiels à la prise de décisions par les responsables :

- Origine des avantages prévus : indications permettant de déterminer s'ils résultent d'un meilleur accès aux marchés étrangers (grâce à des mesures de libéralisation dans d'autres pays) ou d'une contestabilité et d'une efficacité accrues du marché intérieur, les services étant proposés aux utilisateurs (industrie et consommateurs) à des prix moins élevés grâce à une diminution des coûts de production ou des rentes.

- Contribution spécifique, pour l'économie globale, de la libéralisation de différents secteurs, modes de fourniture de services et facteurs.

- Ampleur de la variation des avantages, suivant que les obstacles aux échanges génèrent des rentes ou font monter les prix.

- Nature et ampleur des coûts d'ajustement probables (en termes de production et d'emploi) liés à la libéralisation des échanges de services.

Les théoriciens prédisent, non sans ambiguïté, que tous les pays bénéficieront de cette libéralisation. En premier lieu, celle-ci influera sur le prix des marchandises au niveau mondial, ce qui donne à penser que l'évolution des termes de l'échange pourrait être pénalisante pour certains pays. Deuxièmement, compte tenu du caractère fragmenté de cette libéralisation, les différentes catégories d'obstacles au commerce et le déroulement du processus pourraient avoir des répercussions sur les chances qu'elle puisse se révéler avantageuse pour tous les pays. En particulier, la répartition des rentes revenant aux fournisseurs qui bénéficient de mesures protectrices pourrait influer sur les résultats en matière de bien-être. De nombreux économistes tendent à écarter ce type de préoccupations, qu'ils jugent intéressantes sur un plan théorique mais sans réelle importance pratique. Les modèles EGC pourraient contribuer à en évaluer la pertinence, en prenant en considération les aspects suivants :

- Nature et portée de l'évolution concernant la répartition de la production et des revenus entre les différents pays, et en direction des pays qui améliorent leur efficacité. 
- Conséquences liées à l'amélioration de l'efficacité dans un pays donné (mesure dans laquelle l'efficacité accrue de l'économie se répercute sur le revenu national grâce à une diminution du prix des services produits dans le pays par des prestataires nationaux ou étrangers).

- Effets secondaires (négatifs) possibles, notamment :

- appauvrissement (rare, toutefois, dans le cas du commerce de marchandises) ;

- interactions avec l'épargne et l'investissement à l'échelon national ;

- détérioration des termes de l'échange.

Les indications susceptibles de découler de simulations EGC sont à la fois très diverses et riches d'enseignements. Elles présentent néanmoins trois grandes limitations: (a) elles ne fournissent pas d'analyse sectorielle; (b) à ce jour, la plupart d'entre elles recensent exclusivement les gains statiques et ponctuels générés par l'élimination ou la réduction des obstacles aux échanges de services (*); (c) compte tenu de la quantité importante d'informations nécessaires et du fait que les modèles EGC s'appuient sur des cadres théoriques eux-mêmes fondés sur de nombreuses hypothèses, les résultats ne sont pas toujours fiables.

Note :

La description ci-dessus fait référence aux études EGC disponibles. Il convient cependant de souligner que rien, dans la nature même des modèles calibrés, ne les confine à des effets de niveau ou à certains secteurs de services agrégés. Les résultats de ces simulations s'expriment en niveaux si les intrants se présentent sous cette forme. L'étude d'un modèle qui utiliserait des données de croissance fournirait des résultats libellés en termes de croissance également. Quant à la question d'une analyse par secteur, l'une des premières priorités pour les travaux futurs sera d'élaborer des modèles distincts pour les différents secteurs de services et d'examiner les priorités sectorielles en matière de libéralisation. 


\section{Appendice 3. Études économétriques}

Il existe deux types d'études économétriques :

- Les études réalisées à l'échelle de l'économie, qui permettent de quantifier les effets globaux des obstacles aux échanges de services sur certaines mesures de performances pour l'ensemble de l'économie. Il peut s'agir d'effets de niveau (si la mesure des performances est exprimée en niveaux) ou d'effets de croissance (si cette mesure est exprimée sous la forme de taux de croissance). Ces études ont un objectif similaire à celui des simulations EGC. Toutefois, alors que ces dernières optent pour une approche structurelle afin de montrer comment les barrières imposées dans un secteur donné ont également des incidences sur d'autres secteurs et sur l'économie dans son ensemble, les études économétriques se caractérisent par l'approche de la forme réduite Par conséquent, la comparaison entre études économétriques et modèles EGC s'articule autour des différences entre approche structurelle et méthode de la forme réduite. Parmi les problèmes figurent la testabilité et le contenu des informations, de même que la nécessité que les études économétriques permettent de contrôler la totalité des autres facteurs ayant une incidence sur les performances, ainsi que de traiter (le cas échéant) les questions de simultanéité.

- Les études sectorielles, qui ont pour objet de quantifier les effets directs des obstacles aux échanges de services sur les mesures de performances sectorielles. Il peut s'agir d'effets de niveau (si la mesure des performances est exprimée en niveaux) ou d'effets de croissance (si cette mesure est exprimée sous la forme de taux de croissance). Pour bien interpréter ces études, il faut tenir compte du fait qu'elles sont sectorielles, et ne visent donc pas à une évaluation globale des répercussions des barrières au commerce de services à l'échelle de l'économie, comme le font les études EGC. Au contraire, les résultats des études économétriques sectorielles concernant les effets directs fournissent les principaux éléments indispensables aux études EGC, qui recherchent ensuite les incidences de ces obstacles sur d'autres secteurs de l'économie et, en cas d'approche désagrégée, peuvent aussi combiner les effets de ces obstacles sur différents secteurs de services. 


\section{Appendice 4. Résultats de l'évaluation des restrictions touchant les échanges de services bancaires ${ }^{\mathrm{a}}$}

\begin{tabular}{|c|c|c|c|c|c|c|c|c|}
\hline \multirow[t]{2}{*}{ Économie } & \multicolumn{2}{|c|}{ McGuire et Schuele (2000) } & \multicolumn{2}{|c|}{ Mattoo et al (2001) $\mathbf{b}$} & \multicolumn{2}{|c|}{ Mattoo 1998} & \multicolumn{2}{|c|}{$\begin{array}{c}\text { Claessens et Glaessner 1998, } \\
\text { McGuire } 1998 \text { et McGuire et } \\
\text { Schuele } 1999^{c}\end{array}$} \\
\hline & Note & Classement & Note & Classement & Note & Classement & Note & Classement \\
\hline Malaisie & 0.65 & 1 & 0.44 & 2 & 0.84 & 2 & 0.52 & 2 \\
\hline Inde & 0.60 & 2 & 0.44 & 2 & 0.80 & 3 & 0.55 & 1 \\
\hline Indonésie & 0.55 & 3 & 0.22 & 4 & 0.72 & 5 & 0.36 & 7 \\
\hline Philippines & 0.53 & 4 & 0.44 & 2 & 0.76 & 4 & 0.33 & 10 \\
\hline Brésil & 0.51 & 5 & 0.44 & 2 & 0.80 & 3 & n.c. & s.o. \\
\hline Uruguay & 0.46 & 6 & 0.56 & 1 & 0.80 & 3 & n.c. & s.o. \\
\hline Rép. de Corée & 0.43 & 7 & 0.44 & 2 & 0.80 & 3 & 0.41 & 5 \\
\hline Chili & 0.40 & 8 & 0.22 & 4 & 0.80 & 3 & 0.44 & 4 \\
\hline Thaïlande & 0.39 & 9 & 0.44 & 2 & 0.92 & 1 & 0.36 & 7 \\
\hline Turquie & 0.37 & 10 & 0.11 & 5 & 0.40 & 8 & n.c. & s.o. \\
\hline Singapour & 0.37 & 10 & 0.11 & 5 & 0.64 & 6 & 0.50 & 3 \\
\hline Colombie & 0.23 & 11 & 0.22 & 4 & 0.80 & 3 & 0.35 & 8 \\
\hline Japon & 0.19 & 12 & n.c. & s.o. & 0.16 & 13 & 0.34 & 9 \\
\hline Afrique du Sud & 0.19 & 12 & 0.11 & 5 & 0.20 & 12 & n.c. & s.o. \\
\hline Venezuela & 0.17 & 13 & 0.56 & 1 & 0.80 & 3 & n.c. & s.o. \\
\hline Mexique & 0.17 & 13 & 0.33 & 3 & 0.60 & 7 & 0.38 & 6 \\
\hline Australie & 0.12 & 14 & 0.11 & 5 & 0.27 & 11 & 0.16 & 12 \\
\hline Hong Kong Chine & 0.09 & 15 & 0.11 & 5 & 0.37 & 9 & 0.05 & 15 \\
\hline Suisse & 0.08 & 16 & 0.11 & 5 & 0.16 & 13 & n.c. & s.o. \\
\hline Canada & 0.07 & 17 & 0.11 & 5 & 0.36 & 10 & 0.20 & 11 \\
\hline $\mathrm{UE}^{\mathbf{d}}$ & 0.07 & 17 & 0.11 & 5 & 0.36 & 10 & n.c. & s.o. \\
\hline Argentine & 0.07 & 17 & 0.11 & 5 & 0.16 & 13 & n.c. & s.o. \\
\hline États-Unis & 0.06 & 18 & 0.11 & 5 & 0.36 & 10 & 0.10 & 13 \\
\hline N.-Zélande & 0.06 & 18 & 0.11 & 5 & 0.16 & 13 & 0.08 & 14 \\
\hline
\end{tabular}

n.c. - non calculé ; n.a. - sans objet. ${ }^{\mathbf{a}}$ Le taux de restrictivité des échanges est compris entre 0 et 1 . Plus le chiffre est élevé, plus le régime est restrictif. Les taux d'ouverture des marchés de Claessens et Glaessner (1998), Mattoo (1998), Mattoo et al (2001) et McGuire (1998) ont été convertis en taux de restrictivité et adaptés à la note maximale de 1 afin de permettre les comparaisons. ${ }^{\mathbf{b}}$ L'étude de Mattoo et al (2001) couvre les services financiers ; ces résultats sont utilisés également pour les services bancaires. ${ }^{\mathbf{c}}$ McGuire (1998) et McGuire et Schuele (1999) ont élargi la portée des résultats de Claessens et Glaessner (1998). ${ }^{\mathbf{d}}$ Le chiffre relatif aux 15 États membres de l'UE était le même dans les différentes études.

Source: McGuire (2002). 
Appendice 5. Taux de restrictivité des échanges de services pour des secteurs particuliers, dans des économies en développement, des pays à revenu faible ou moyen (prfm), et des pays à revenu élevé (PRE)

\section{Services bancaires ${ }^{a}$}

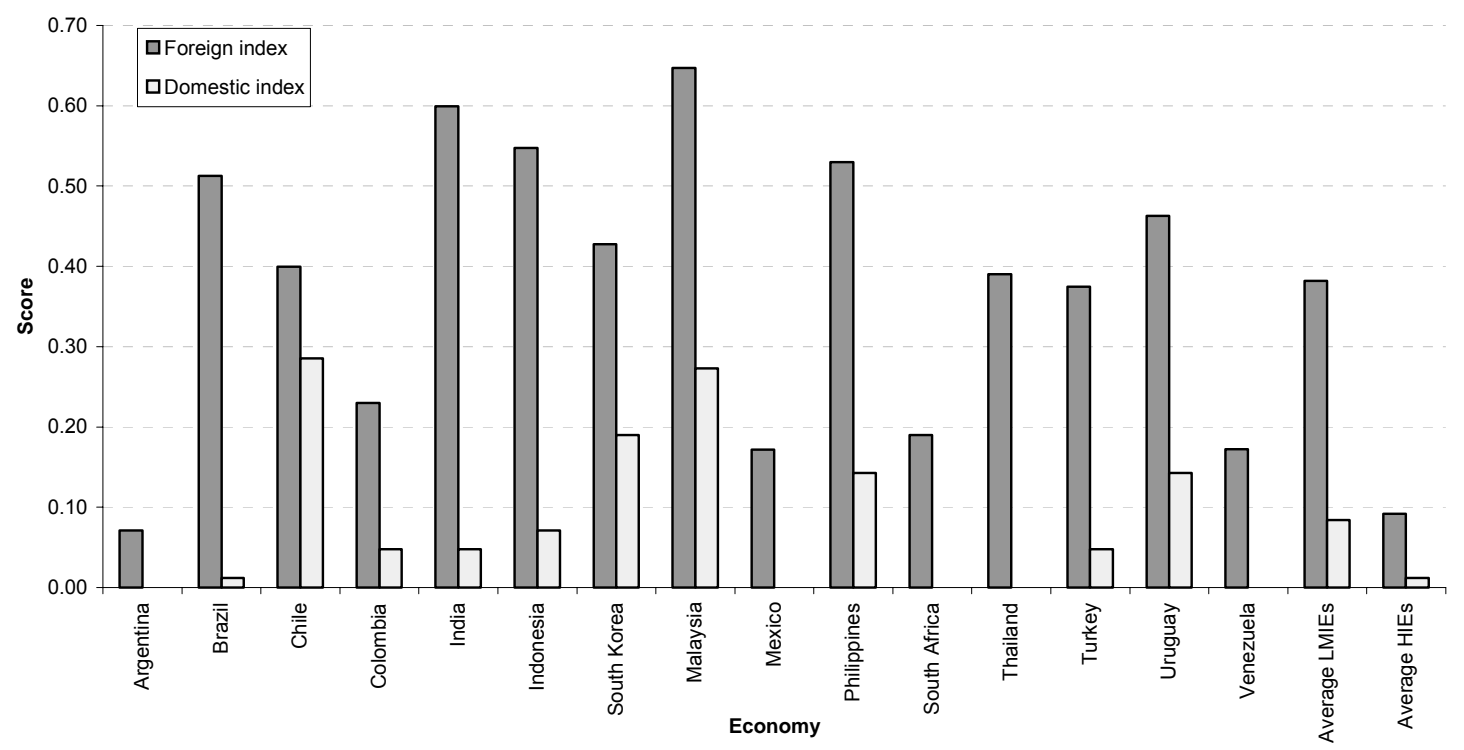

a D'après les informations disponibles concernant les restrictions en place au 31 décembre 1997.

b L'indice de restrictivité est compris entre 0 et 1. plus la note est élevée, plus l'économie est restrictive Source : McGuire et Schuele (2000), mentionné dans McGuire (2002). 
TD/TC/WP(2003)23/FINAL

\section{Services de distribution ${ }^{a}$}

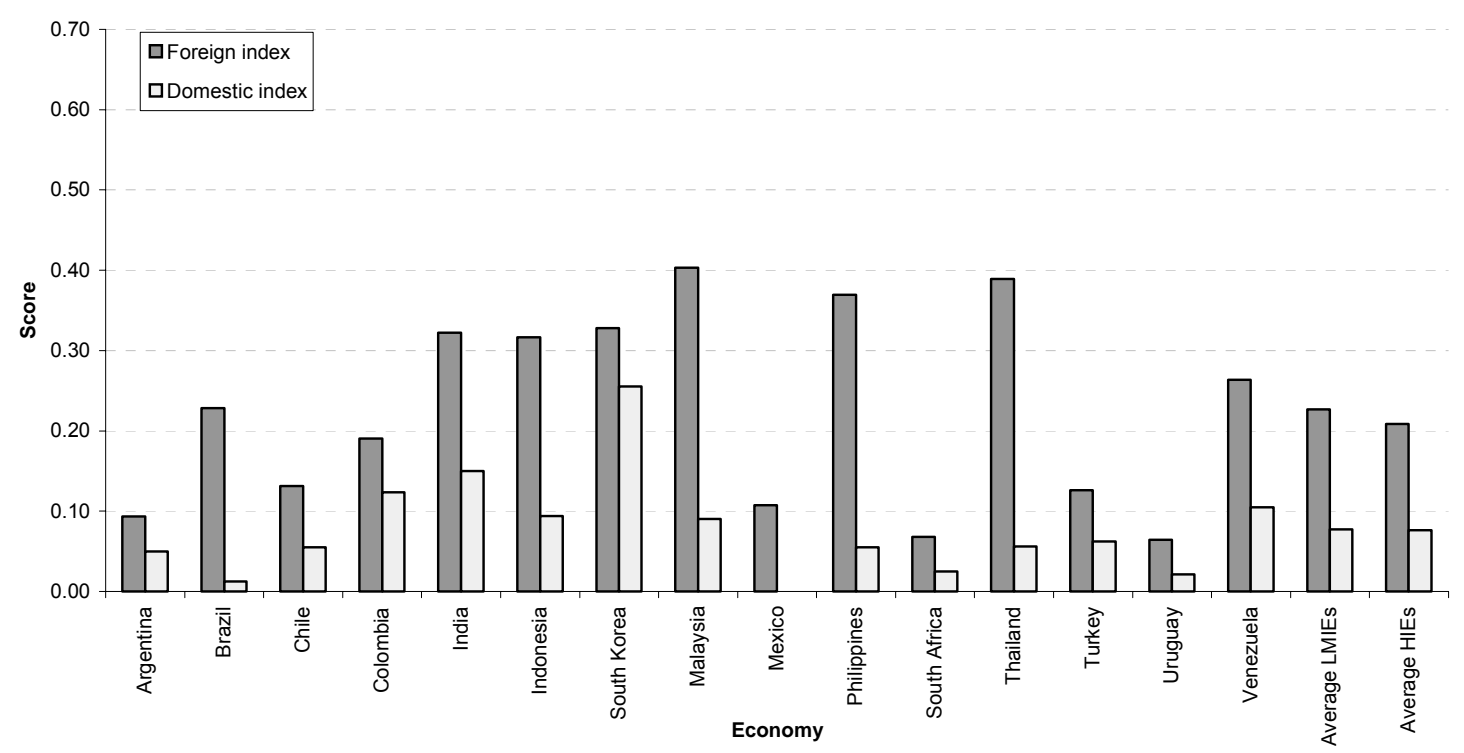

a D'après les informations disponibles concernant les restrictions en place au 30 juin 1999.

b L'indice de restrictivité est compris entre 0 et 1 . Plus la note est élevée, plus l'économie est restrictive.

Source : Kalirajan (2000), mentionné dans McGuire (2002).

\section{Services maritimes ${ }^{a}$}

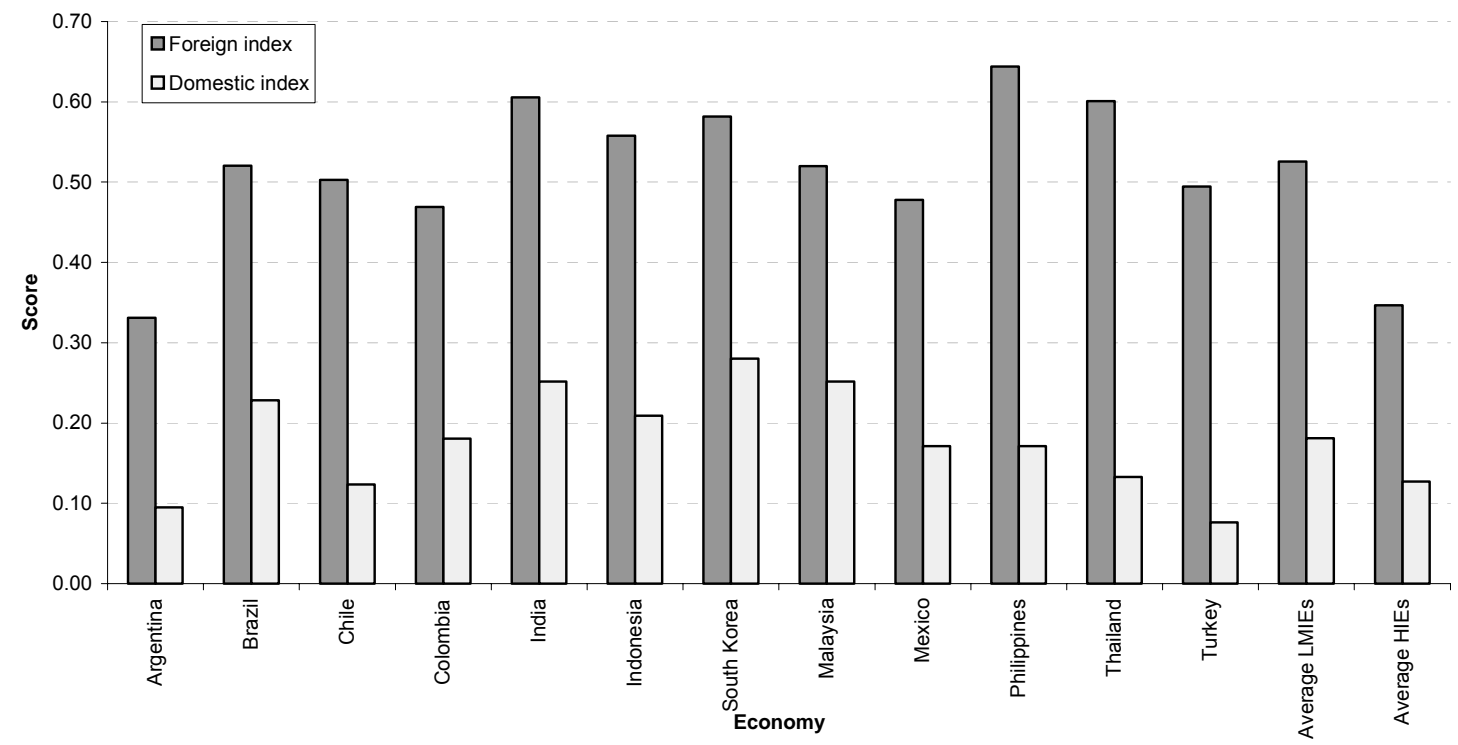

a D'après les informations disponibles concernant les restrictions en place au 31 décembre 1998.

b L'indice de restrictivité est compris entre 0 et 1 . Plus la note est élevée, plus l'économie est restrictive.

Source : McGuire et al (2000), mentionné dans McGuire (2002). 


\section{Services professionnels $a b$}

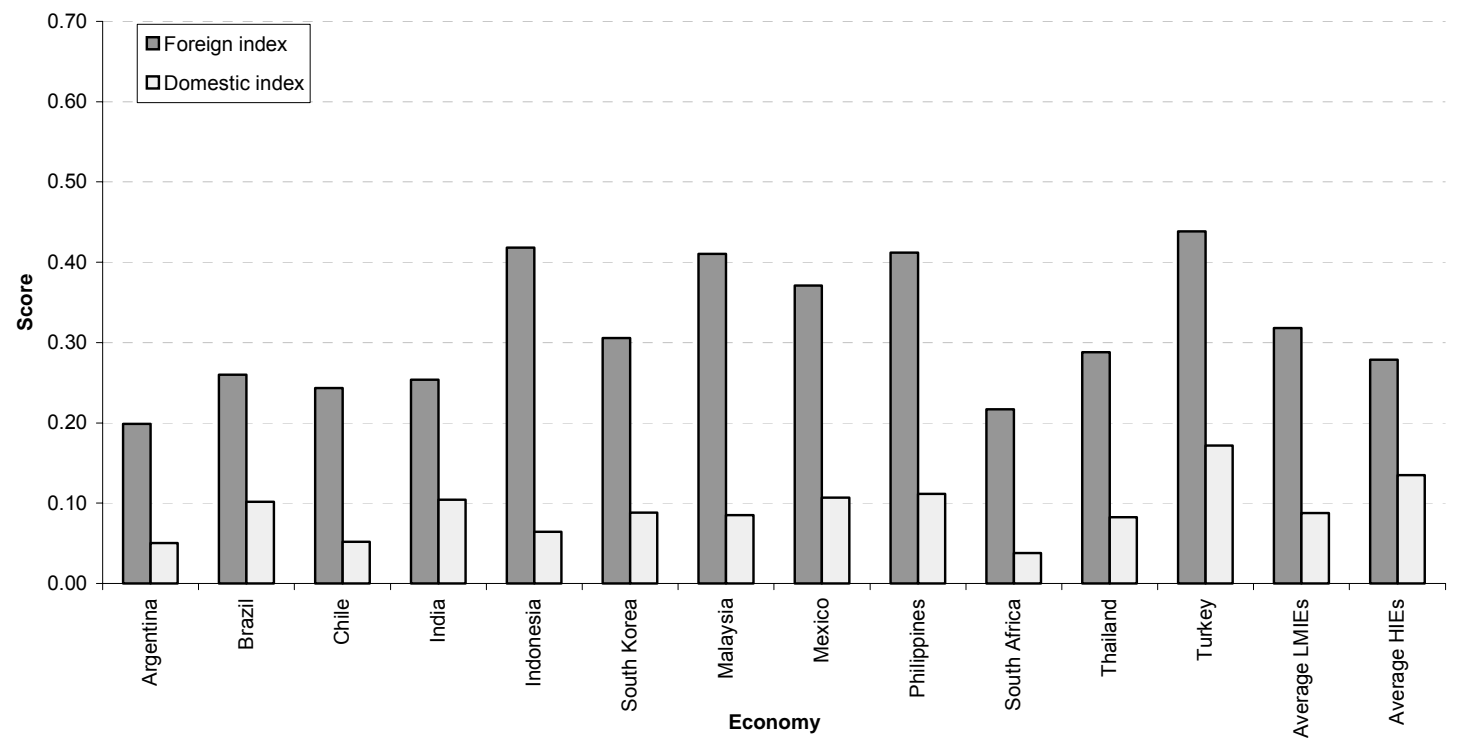

$\overline{\mathbf{a}}$ D'après les informations disponibles concernant les restrictions en place au 30 juin 1999. ${ }^{\mathbf{b}}$ Les résultats relatifs aux services professionnels correspondent à la moyenne des taux de restriction des échanges dans plusieurs secteurs et concernent : comptables, architectes, ingénieurs et juristes. ${ }^{\mathbf{c}}$ L'indice de restrictivité est compris entre 0 et 1 . Plus la note est élevée, plus l'économie est restrictive.

Source : Nguyen-Hong (2001), mentionné dans McGuire (2002).

\section{Services de télécommunications ${ }^{a b}$}

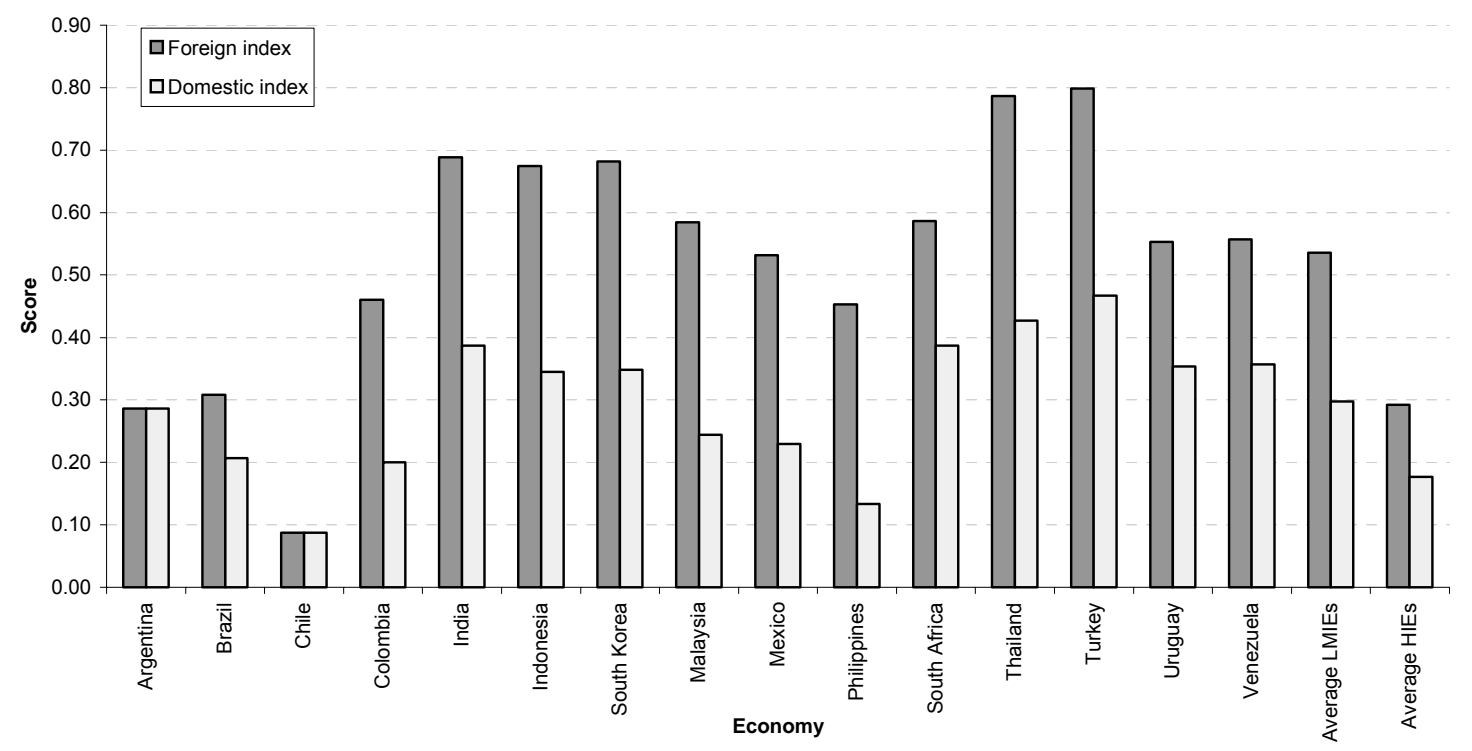

a D'après une étude menée par l'Union internationale des télécommunications (UIT 1999). b Calculs effectués à partir des travaux de Warren (2000a). Ce tableau donne un exemple de taux de restriction des échanges. Warren a également fait des calculs pour d'autres économies en développement. ${ }^{\mathbf{c}}$ L'indice de restrictivité est compris entre 0 et 1 . Plus la note est élevée, plus l'économie est restrictive.

Source : Adapté de Warren (2000a) par McGuire (2002). 
Appendice 6. Résultats d'études egc modélisant les services sous forme d'échanges transfrontières - mode 1 de fourniture de services

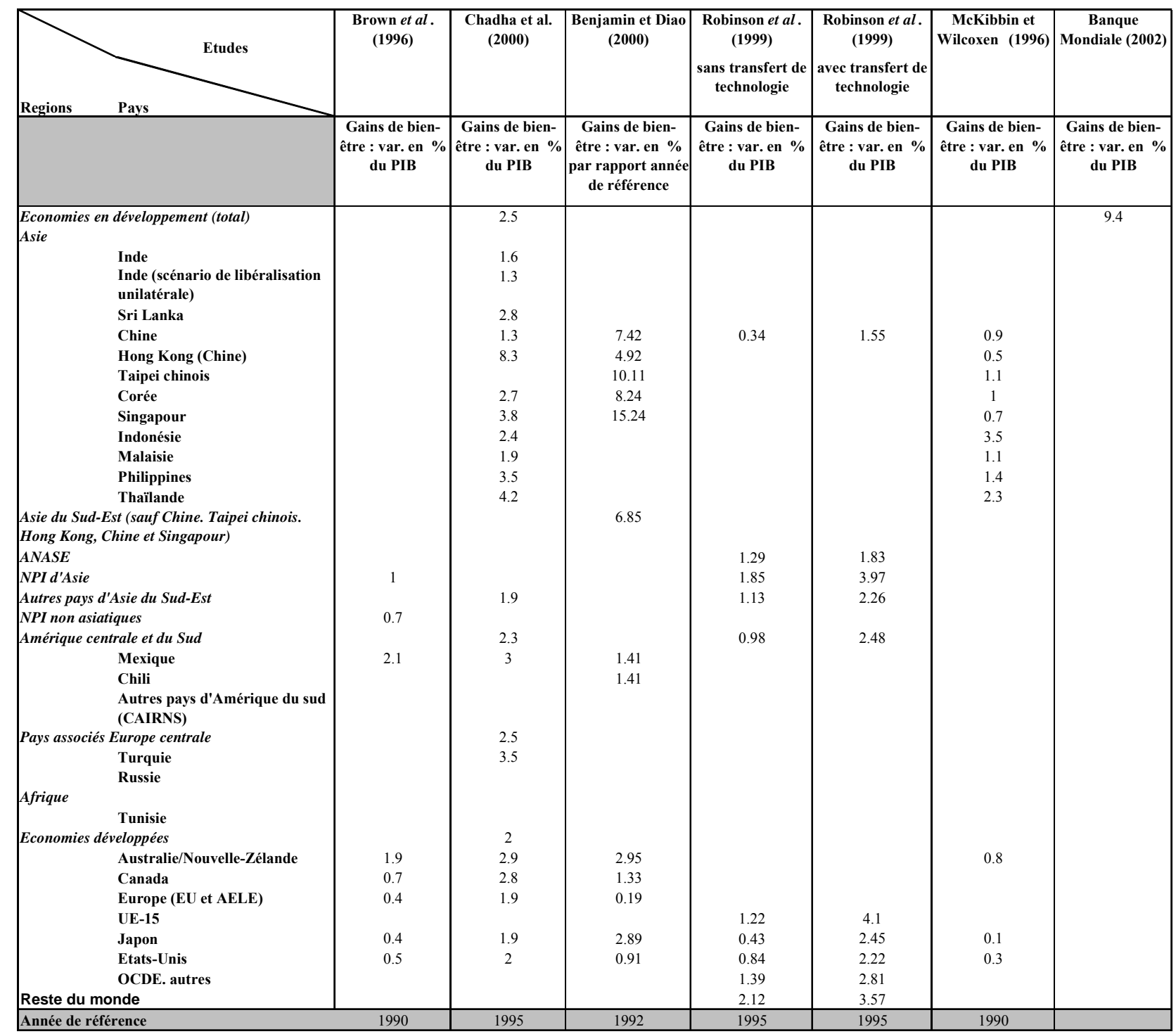


Appendice 7. Résultats d'études egc modélisant séparément les obstacles à la présence commerciale - mode 3 de fourniture de services - et ceux qui concernent les autres modes

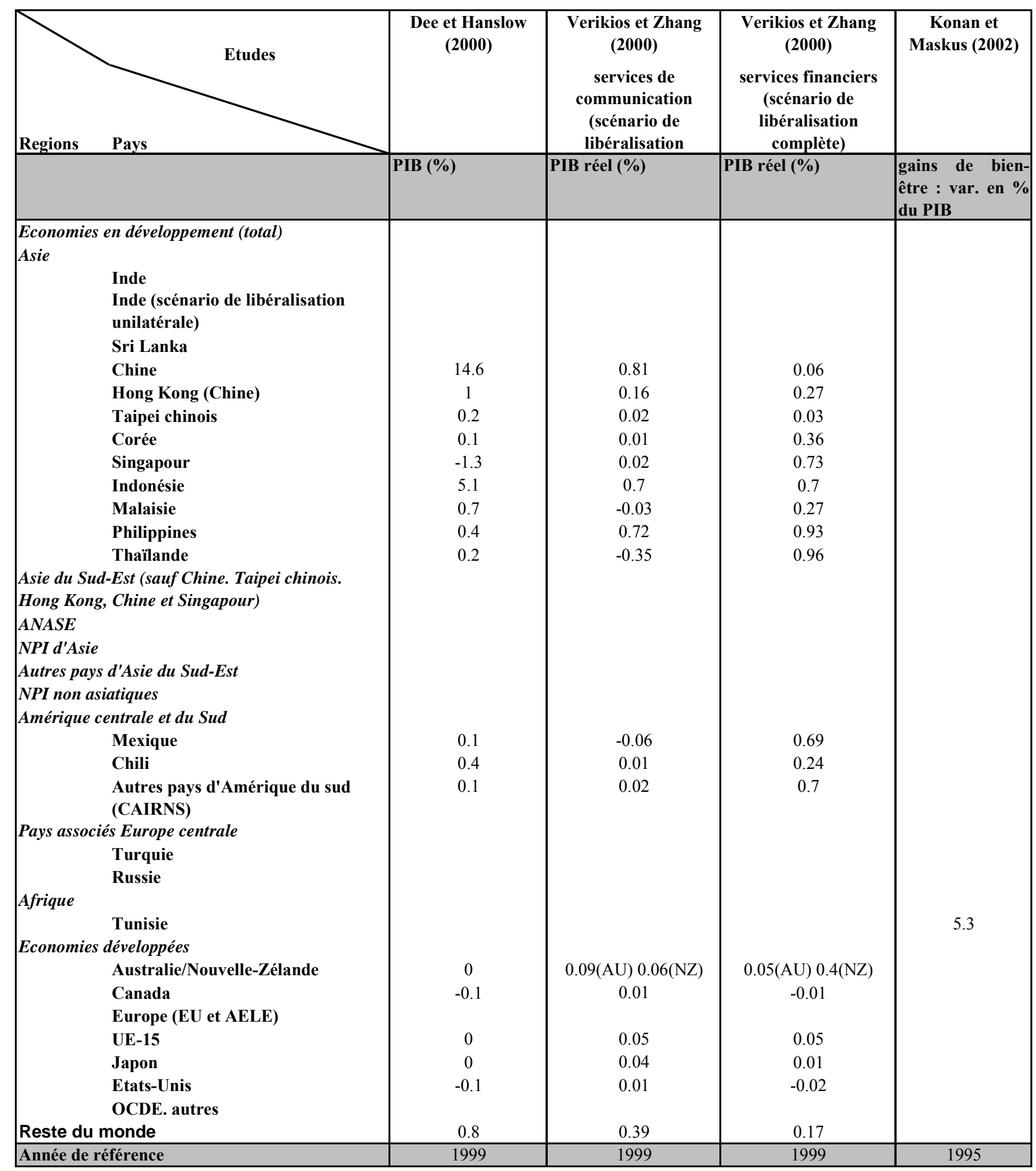




\section{Appendice 8. Sélection d'études économétriques}

\begin{tabular}{|c|c|c|}
\hline Publication & \multicolumn{2}{|c|}{$\begin{array}{l}\text { Claessens S., Demirgüç-Kunt A. et Huizinga H. "How does foreign entry affect domestic banking } \\
\text { markets?" Journal of Banking and Finance 25, pp. 891-911. }\end{array}$} \\
\hline $\begin{array}{l}\text { Couverture } \\
\text { géographique }\end{array}$ & \multicolumn{2}{|l|}{7900 banques de 80 pays du monde entier } \\
\hline Période & \multicolumn{2}{|l|}{ 1988-1995 } \\
\hline $\begin{array}{l}\text { Couverture } \\
\text { sectorielle }\end{array}$ & \multicolumn{2}{|l|}{ Services bancaires commerciaux } \\
\hline $\begin{array}{l}\text { Méthode } \\
\text { d'évaluation de la } \\
\text { libéralisation }\end{array}$ & \multicolumn{2}{|c|}{$\begin{array}{l}\text { estimation effectuée selon la méthode des moindres carrés en tenant compte des effets fixes de pays et de } \\
\text { temps, afin de mesurer l'impact de la participation de banques étrangères sur différents indicateurs de } \\
\text { l'efficience bancaire d'un pays. Les estimations s'accompagnent d'un contrôle de tous les facteurs } \\
\text { financiers et macroéconomiques standard intervenant dans les relations étudiées, à savoir: }\end{array}$} \\
\hline $\begin{array}{l}\text { Structure du } \\
\text { modèle }\end{array}$ & \multicolumn{2}{|c|}{$\begin{array}{l}\Delta \mathrm{I}_{\mathrm{ijt}}=\alpha_{0}+\alpha_{1} \Delta \mathrm{FS}_{\mathrm{it}}+\alpha_{2 \mathrm{i}} \Delta \mathrm{B}_{\mathrm{it}}+\alpha_{3 \mathrm{i}} \Delta \mathrm{X}_{\mathrm{it}}+\varepsilon_{\mathrm{ijt}} \quad \text { sachant que : } \\
\text { - } \quad \mathrm{I}_{\mathrm{itt}} \text { est la variable dépendante (soit: } \Delta \text { actifs non porteurs d'intérêts-total des actifs, } \Delta \text { bénéfices } \\
\text { avant impôt/total des actifs ou } \Delta \text { frais généraux) pour une banque nationale } i \text { dans un pays } j \text { à une } \\
\text { date } t \text {. } \\
\text { - } \quad \mathrm{FS}_{\mathrm{it}} \text { représente la proportion de pays étrangers dans les systèmes bancaires nationaux } \\
\text { - } \quad \mathrm{B}_{\mathrm{it}} \text { représente les variables banque pour une banque nationale } i \text { à une date } t \\
\text { - } \quad \mathrm{X}_{\mathrm{it}} \text { représente les variables pays pour un pays } j \text { à une date } t\end{array}$} \\
\hline $\begin{array}{l}\text { Année de } \\
\text { référence/source } \\
\text { des données }\end{array}$ & \multicolumn{2}{|c|}{ Base de données DATASCOPE, couvrant approximativement $90 \%$ des actifs bancaires dans chaque pays. } \\
\hline \multirow[t]{5}{*}{ Résultats } & \multicolumn{2}{|c|}{$\begin{array}{l}\text { Le nombre de banques étrangères est lié à une réduction : } \\
\text { - de la rentabilité des banques nationales (concurrence) } \\
\text { - des revenus non porteurs d'intérêt (efficacité de la gestion et structure organique) } \\
\text { Aucun changement notable n'est enregistré dans les marges d'intérêt nettes ou les provisions pour pertes sur } \\
\text { prêts. } \\
\text { Interprétation: la présence de banques étrangères est associée à une amélioration de l'efficacité des systèmes } \\
\text { bancaires nationaux. }\end{array}$} \\
\hline & Variable dépendante & $\begin{array}{l}\text { Évolution }(\Delta) \text { de la proportion de banques étrangères } \\
\text { (variable explicative) }\end{array}$ \\
\hline & $\Delta$ revenus non porteurs d'int. / tot.actifs & $-0.023^{*}$ \\
\hline & $\Delta$ bénéfices avant impôt/ tot.actifs & $-0.028^{* *}$ \\
\hline & $\begin{array}{l}\Delta \text { frais généraux/ tot.actifs } \\
\text { Note: }{ }^{* * *},{ }^{* * *} \text { incidence statistique à } 10 \%, 5 \% \text { et } 1\end{array}$ & $\begin{array}{l}-0.015^{*} \\
\text { spectivement. }\end{array}$ \\
\hline Mise en garde & \multicolumn{2}{|c|}{$\begin{array}{l}\text { S'il est vrai que ces indicateurs ne permettent qu'une mesure imparfaite de l'efficacité et peuvent comporter } \\
\text { des données « parasites », les mesures présentées ici semblent exemptes de toute distorsion systématique. }\end{array}$} \\
\hline
\end{tabular}




\section{Appendice 8. (suite)}

\begin{tabular}{|c|c|c|c|}
\hline Publication & \multicolumn{3}{|c|}{$\begin{array}{l}\text { Eschenbach, F. \& Francois, J., 2002. "Financial Sector Competition, Services Trade, and Growth," } \\
\text { CEPR Discussion Papers 3573. }\end{array}$} \\
\hline $\begin{array}{l}\text { Couverture } \\
\text { géographique }\end{array}$ & \multicolumn{3}{|c|}{130 pays répartis dans le monde entier } \\
\hline Période & \multicolumn{3}{|l|}{ 1990-1999 } \\
\hline $\begin{array}{l}\text { Couverture } \\
\text { sectorielle }\end{array}$ & \multicolumn{3}{|l|}{ Services financiers } \\
\hline $\begin{array}{l}\text { Méthode } \\
\text { d'évaluation des } \\
\text { obstacles }\end{array}$ & \multicolumn{3}{|c|}{ Équivalents tarifaires de Hoekman (1995) } \\
\hline $\begin{array}{l}\text { Méthode } \\
\text { d'évaluation de la } \\
\text { libéralisation }\end{array}$ & \multicolumn{3}{|c|}{$\begin{array}{l}\text { Modèle analytique fondé sur le principe d'accumulation de Ramsey et sur l'hypothèse d'un marché } \\
\text { oligopolistique, l'objectif étant de mettre en évidence les voies par lesquelles la libéralisation des services } \\
\text { financiers pourrait dynamiser l'économie et favoriser la concurrence. Les estimateurs utilisés s'appuient sur } \\
\text { la correction d'erreur simultanée (approche similaire à l'estimation itérative en trois étapes) dans le modèle } \\
\text { empirique afin d'établir des régressions au sujet de la croissance dans différents pays. Des variables } \\
\text { standard sont utilisées comme éléments de contrôle. }\end{array}$} \\
\hline $\begin{array}{l}\text { Structure du } \\
\text { modèle }\end{array}$ & \multicolumn{3}{|c|}{$\begin{array}{l}\text { Système faisant intervenir simultanément trois équations, dont les variables dépendantes sont } \\
\text { respectivement les suivantes: (1) concentration (part des trois banques les plus importantes), (2) marges } \\
\text { bénéficiaires (mesurées en tant que revenu net d'intérêts-NIM) ou taux de rentabilité des banques (PROFIT) } \\
\text { et (3) croissance par habitant: } \\
\qquad \operatorname{CONCENT}_{\mathrm{i}}=\mathbf{A X}_{1, \mathrm{i}}+\varepsilon_{1, \mathrm{i}} \\
\qquad \mathrm{NIM}_{\mathrm{i}}\left(\mathrm{ou}_{\mathrm{PROFIT}} \mathrm{PRX}_{2, \mathrm{i}}+\varepsilon_{2, \mathrm{i}}\right. \\
\qquad \mathrm{PCGR}_{\mathrm{i}}=\mathbf{C X}_{3, \mathrm{i}}+\varepsilon_{3, \mathrm{i}} \\
\text { Les variables du deuxième élément des équations comprennent : } \\
\text { - équation 1: taille, équivalent tarifaire selon Hoekman (95), proportion de banques étrangères } \\
\text { dans le secteur bancaire, évaluation du degré d'ouverture du système bancaire, une variable factice } \\
\text { pour les économies en transition } \\
\text { - équation 2: mesure de la concentration du secteur, un indicateur de crise financière et une } \\
\text { variable factice pour les économies en transition } \\
\text { - équation 3: PIB par habitant en 1990, croissance démographique, échanges commerciaux sous } \\
\text { forme de part du PIB, taux d'inflation moyen, revenu net d'intérêts, bénéfices, un indicateur de } \\
\text { stabilité politique et la variable factice pour les économies en transition. }\end{array}$} \\
\hline Source & \multicolumn{3}{|c|}{ BANKSCOPE, FMI, Banque mondiale, Heritage Foundation index of Economic Freedom } \\
\hline \multirow[t]{8}{*}{ Résultats } & \multicolumn{3}{|c|}{$\begin{array}{l}\text { Lien positif étroit entre concurrence et ouverture dans le secteur financier, et entre concurrence dans le } \\
\text { secteur financier et croissance. }\end{array}$} \\
\hline & Variable dépendante & Variable explicative & Coefficient \\
\hline & CONCENT & Équivalent tarifaire & $0.693^{* * *}$ \\
\hline & CONCENT & Proportion banques étr. & $-1.202^{* * *}$ \\
\hline & CONCENT & Restrictivité secteur banc. & $14.515^{* * *}$ \\
\hline & PUISSANCE COMM. (NIM) & Concentration & $0.063^{* * *}$ \\
\hline & CROISSANCE PIB PAR HAB. & Marges nettes d'intérêts & $-0.469^{* * *}$ \\
\hline & & & $-1.078^{* * *}$ \\
\hline Mise en garde & \multicolumn{3}{|c|}{ - Couverture irrégulière pour certains des indicateurs } \\
\hline $\begin{array}{l}\text { Zoom sur les pays } \\
\text { en développement }\end{array}$ & \multicolumn{3}{|c|}{$\begin{array}{l}\text { Il apparaît que les économies protégées sont concentrées dans les pays à faible revenu. Indépendamment } \\
\text { d'autres éléments statistiques pertinents et toutes choses constantes par ailleurs, si un pays à faible revenu } \\
\text { représentatif de cette catégorie devait passer de la situation d'ouverture moyenne qui caractérise ces pays à } \\
\text { un régime plus libéral, et donc plus ouvert, plutôt caractéristique des pays à revenu élevé (soit une } \\
\text { libéralisation d'environ } 50 \% \text { d'après les mesures employées ici), le résultat indiquerait une différence entre } \\
\text { les taux de croissance qui se situerait approximativement entre } 0.4 \text { et } 0.6 \% \text { par an et par habitant. }\end{array}$} \\
\hline
\end{tabular}


TD/TC/WP(2003)23/FINAL

\begin{tabular}{|c|c|}
\hline Publication & $\begin{array}{l}\text { Mattoo A., Rathindran R. et Subramanian A. (2001) "Measuring services trade liberalisation and its } \\
\text { impact on economic growth: an illustration", Banque mondiale }\end{array}$ \\
\hline $\begin{array}{l}\text { Couverture } \\
\text { géographique }\end{array}$ & 60 pays répartis dans le monde entier, dont 37 en développement \\
\hline Période & 1990-1999 \\
\hline $\begin{array}{l}\text { Couverture } \\
\text { sectorielle }\end{array}$ & $\begin{array}{l}\text { - Télécommunications } \\
\text { - Services financiers }\end{array}$ \\
\hline $\begin{array}{l}\text { Méthode } \\
\text { d'évaluation des } \\
\text { obstacles }\end{array}$ & $\begin{array}{l}\text { Les indicateurs tiennent compte des aspects politiques plutôt que des résultats (afin d'inclure dans un seul } \\
\text { indice, suite aux critiques formulées par Rodriguez et Rodrik (1999), trois aspects essentiels de la politique } \\
\text { commerciale : (1) concurrence ou structure du marché, (2) pénétration d'entreprises étrangères, (3) } \\
\text { réglementation. } \\
\text { - Indicateur du degré d'ouverture du secteur des télécommunications: indices allant de } 1 \text { à 9, le } \\
\text { chiffre } 9 \text { étant attribué à un marché qui favorise la concurrence entre fournisseurs, ne limite pas } \\
\text { l'IDE et dispose d'une autorité réglementaire indépendante, et la valeur } 1 \text { à une structure } \\
\text { monopolistique, fermée à l'IDE et dépourvue de réglementation. Un système lexicographique } \\
\text { attribue le coefficient de pondération le plus élevé à la composante définissant le degré de } \\
\text { concurrence, un coefficient intermédiaire à l'indicateur relatif à la présence étrangère et le plus } \\
\text { faible à celui qui concerne la réglementation (ainsi, un régime autorisant la concurrence } \\
\text { obtiendra une meilleure place dans le classement qu'un régime qui l'interdirait, quel que soit le } \\
\text { traitement des deux autres indicateurs). Lorsque la structure du marché et la réglementation } \\
\text { relative à la présence de fournisseurs de services étrangers diffèrent pour les appels } \\
\text { internationaux, les appels nationaux courte distance et les appels nationaux longue distance, } \\
\text { c'est la moyenne pondérée des trois qui est prise en considération. } \\
\text { - Indicateur du degré d'ouverture des services financiers: indices allant de } 1 \text { à } 8 \text {, les valeurs les } \\
\text { plus élevées étant attribuées en cas de libéralisation plus avancée ; le coefficient de pondération } \\
\text { le plus fort s'applique à la structure du marché, le coefficient intermédiaire à la présence } \\
\text { étrangère et le plus faible à la réglementation du commerce transfrontières. }\end{array}$ \\
\hline $\begin{array}{l}\text { Méthode } \\
\text { d'évaluation de la } \\
\text { libéralisation et } \\
\text { structure du modèle }\end{array}$ & $\begin{array}{l}\text { Régressions sur la base de la croissance dans différents pays selon Barro (1977) et Sachs-Warner (1995, } \\
\qquad \mathbf{G}_{\mathbf{i}}=\boldsymbol{\alpha}+\boldsymbol{\beta} \mathbf{X}_{\mathbf{i}}+\gamma \mathbf{R}_{\mathbf{i}}, \quad \text { pour } \mathrm{j}=1, \ldots, \mathrm{N} \\
\text { - } \mathrm{Si} \text { la variable dépendante } \mathrm{Gj} \text { représente la croissance annuelle moyenne du PIB par habitant } \\
\text { - } \mathrm{\alpha}=\text { terme constant } \\
\text { - } \mathrm{Xj}=\text { vecteur des contrôles standard de la croissance pour un pays } \mathrm{j} \\
\text { - } \mathrm{Rj}=\text { vecteur de l'ouverture au commerce de services pour un pays } \mathrm{j}\end{array}$ \\
\hline $\begin{array}{l}\text { Année de } \\
\text { référence/source } \\
\text { des données }\end{array}$ & $\begin{array}{l}\text { - Télécommunications: données provenant d'une base de données Banque mondiale-UIT } \\
\text { récemment créée } \\
\text { - Services financiers: } \\
\text { - Informations sur la politique de la concurrence dans le secteur bancaire et sur la } \\
\text { présence étrangère, obtenues d'après les engagements pris par les pays dans le cadre de } \\
\text { l'AGCS pour ce qui concerne les services financiers, adaptées de manière à tenir } \\
\text { compte de la politique en vigueur sur le mode } 3 \text { et corrigées en fonction de la structure } \\
\text { de marché constatée et du taux de pénétration des banques étrangères. } \\
\text { - Il n'existe pas d'informations adéquates sur la réglementation. Les données } \\
\text { concernant le degré d'ouverture des comptes courants et comptes de capital, qui } \\
\text { renseignent sur les règlements applicables aux modes } 1 \text { et } 2 \text { de prestation de services, } \\
\text { sont fournies par Dailami (2000). }\end{array}$ \\
\hline $\begin{array}{l}\text { Conclusion du } \\
\text { document et } \\
\text { principaux résultats }\end{array}$ & $\begin{array}{l}\text { - La mobilité des facteurs et les effets d'échelle particuliers à la libéralisation des services } \\
\text { attestent qu'il s'agit d'un processus différent de la libéralisation du commerce de marchandises. } \\
\text { - Les différences apparaissent mieux dans les évaluations visant à mesurer le degré d'ouverture } \\
\text { d'après la politique commerciale plutôt qu'en fonction des résultats. } \\
\text { - Preuves économétriques de l'influence sur la croissance de l'ouverture des services (marquée } \\
\text { dans le cas des services financiers, moins forte pour les télécommunications). }\end{array}$ \\
\hline
\end{tabular}


Appendice 8. (suite)

\begin{tabular}{|c|c|c|c|c|c|}
\hline Résultats & $\begin{array}{l}4 \\
3 \\
2 \\
1 \\
\text { Indice d' } \\
\text { Valeur } \\
8\end{array}$ & $\begin{array}{l}\text { ibéralisation des télécommunications } \\
\text { Pays ayant opté pour une } \\
\text { télécommunications - ordre décroissant d } \\
\text { Australie, Canada, Colombie, Républ } \\
\text { Hong Kong Chine, Malaisie, Mexique, Norvé } \\
\text { Chili, République de Corée, Nouvelle-2 } \\
\text { Honduras, Pérou, Sri Lanka } \\
\text { Inde, Indonésie, Venezuela } \\
\text { Argentine, Bolivie, Brésil, Équateur, } \\
\text { Malte, Maurice, Mozambique, Nicaragua, Pa } \\
\text { Jamaïque, Lesotho, Malawi, Thaïlande } \\
\text { Angola, Maroc, Costa Rica } \\
\text { Bénin, Chypre, Gabon, Gambie, Kenya } \\
\text { uverture des services financiers } \\
\text { Pays ayant opté pour une libéralisat } \\
\text { décroissant des indices } \\
\text { Argentine, Australie, Bolivie, Canada } \\
\text { Hong Kong Chine, Islande, Israël, Jamaïq } \\
\text { Nicaragua, Norvège, Panama, Singapour, Afr } \\
\text { Chili, Colombie, Costa Rica, Rép } \\
\text { Honduras, Indonésie, Kenya, Malawi, Malte, } \\
\text { Bahreïn, Mexique } \\
\text { Brésil, Inde, République de Corée, Mal } \\
\text { Pérou, Uruguay, Venezuela } \\
\text { Gabon, Hongrie, Pakistan, Tunisie, Ém }\end{array}$ & $\begin{array}{l}\text { ralisation de } \\
\text { ndices } \\
\text { dominicaine, } \\
\text { Philippines, S } \\
\text { nde } \\
\text { pte, Guyana, } \\
\text { an, Panama, Si } \\
\text { uguay } \\
\text { Inisie, Turquie }\end{array}$ & $\begin{array}{l}\text { services } \\
\text { Salvador, Gh } \\
\text { États-Unis } \\
\text { grie, Islande, Is } \\
\text { our, Afrique du } \\
\text { financiers - or } \\
\text { Salvador, Guy } \\
\text { Nouvelle-Zéla } \\
\text { Irquie, États-Un } \\
\text { Équateur, Gh } \\
\text { Sénégal, Zimba }\end{array}$ & $\begin{array}{l}\text { de } \\
\text { ana, } \\
\text { raël, } \\
\text { Sud }\end{array}$ \\
\hline $\begin{array}{l}\text { Résultats des } \\
\text { régressions }\end{array}$ & \multicolumn{5}{|c|}{$\begin{array}{l}\text { - Le coefficient de pondération de l'indice de libéralisation des télécommunications est positif et } \\
\text { significatif au niveau de } 10 \% \text {. Il prévoit, pour les pays ayant partiellement libéralisé leurs } \\
\text { services de télécommunications, une croissance plus rapide (jusqu'à } 0.15 \% \text { de différence) que } \\
\text { dans les pays où le marché des télécommunications a une structure monopolistique, où les } \\
\text { entrées d'IDE sont interdites et où il n'existe pas d'autorité réglementaire. Les pays ayant } \\
\text { procédé à une libéralisation totale des services de télécommunications tendent à enregistrer une } \\
\text { croissance jusqu'à } 1 \% \text { plus rapide que les autres. } \\
\text { Le coefficient de pondération de l'indice de libéralisation du secteur financier est positif et } \\
\text { significatif au niveau de } 5 \% \text {. Il prévoit, pour les pays ayant partiellement libéralisé leurs } \\
\text { services financiers, une croissance plus rapide (jusqu'à } 0.25 \% \text { de différence) que dans les pays } \\
\text { où le secteur financier est fermé. Les pays ayant procédé à une libéralisation totale de leurs } \\
\text { - } \quad \text { Lervices financiers tendent à enregistrer une croissance jusqu'à } 1.2 \% \text { plus rapide que les autres. } \\
\text { Le coefficient de pondération de l'indice de libéralisation des deux secteurs est significatif mais } \\
\text { négatif. Il montre que, dans les pays associant un certain degré de libéralisation des services de } \\
\text { télécommunications et des services financiers, la croissance est plus lente (jusqu’à } 0.04 \% \text { de } \\
\underline{\text { différence) que dans les pays où ces services ne sont pas libéralisés. Lorsque la libéralisation est }} \\
\text { complète dans les deux secteurs, toutefois, la croissance est plus rapide (jusqu’à } 1.5 \% \text { de } \\
\text { différence). }\end{array}$} \\
\hline
\end{tabular}


TD/TC/WP(2003)23/FINAL

\section{Appendice 8. (suite)}

\begin{tabular}{|c|c|}
\hline & $\begin{array}{l}\text { L'importance des coefficients appliqués aux indices est beaucoup plus élevée pour les régressions qui } \\
\text { portent exclusivement sur les } 37 \text { pays en développement que pour celles qui concernent l'échantillon } \\
\text { complet (60 pays). } \\
\text { - Le coefficient de pondération de l'indice de libéralisation des télécommunications est positif et } \\
\text { significatif au niveau de } 10 \% \text {. Il prévoit, pour les pays en développement ayant partiellement } \\
\text { libéralisé leurs services de télécommunications, une croissance plus rapide (jusqu'à 0.32\% de } \\
\text { différence) que dans les pays en développement où le marché des télécommunications a une } \\
\text { structure monopolistique, où les entrées d'IDE sont interdites et où il n'existe pas d'autorité } \\
\text { réglementaire. Les pays en développement ayant procédé à une libéralisation totale des services } \\
\text { de télécommunications tendent à enregistrer une croissance jusqu'à } 2 \% \text { plus rapide que les } \\
\text { autres pays en développement. Ces pourcentages sont deux fois plus élevés que pour } \\
\text { l'échantillon complet de 60 pays. } \\
\text { Le coefficient de pondération de l'indice de libéralisation du secteur financier est positif et } \\
\text { significatif au niveau de } 5 \% \text {. Il prévoit, pour les pays en développement ayant partiellement } \\
\text { libéralisé leurs services financiers, une croissance plus rapide (jusqu'à 0.36\% de différence) } \\
\text { que dans les pays en développement où le secteur financier est fermé. Les pays en } \\
\text { développement ayant procédé à une libéralisation totale de leurs services financiers tendent à } \\
\text { enregistrer une croissance jusqu'à } 2.3 \% \text { plus rapide que les autres. Ces deux pourcentages } \\
\text { étaient plus faibles pour l'échantillon incluant les pays développés. } \\
\text { Le coefficient de pondération de l'indice de libéralisation des deux secteurs est significatif, mais } \\
\text { négatif. Il montre que, dans les pays en développement associant un certain degré de } \\
\text { libéralisation des services de télécommunications et des services financiers, la croissance est } \\
\text { plus lente (près de 0.04\% de différence) que dans ceux où ces services ne sont pas libéralisés. } \\
\text { Lorsque la libéralisation est complète dans les deux secteurs, toutefois, la croissance est plus } \\
\text { rapide de 2.8\%. }\end{array}$ \\
\hline Mise en garde & $\begin{array}{l}\text { - La qualité des indices d'ouverture doit être améliorée par l'utilisation de données plus fiables et } \\
\text { par une meilleure compréhension de l'interaction entre les composantes de la politique } \\
\text { commerciale - mesures concernant la présence de fournisseurs étrangers, investissement } \\
\text { étranger, réglementation - dans différents secteurs de services. } \\
\text { Les variables relatives à la libéralisation sont construites sur la base d'informations datant de la } \\
\text { seconde moitié des années } 1990 \text {. Il se peut, de ce fait, que les estimations comprennent certains } \\
\text { bénéfices non répétitifs. }\end{array}$ \\
\hline
\end{tabular}




\section{BIBLIOGRAPHIE}

Accenture, Markle Foundation, UNDP Creating a development dynamic: final report of the digital opportunity initiative.

APEC (2000), "Measures Affecting Trade and Investment in Education Services in the Asia-Pacific Region", Singapour.

Banque mondiale (2002), "Global Economic Prospects and the Developing Countries 2002: Making Trade work for the Poor".

Benjamin, N. et Diao, X. (2000), "Liberalising Services Trade in APEC: A General Equilibrium Analysis with Imperfect Competition”, Pacific Economic Review 5:1, pp. 49-75.

Boylaud, O. et Nicoletti, G. (2000), "Regulation, market structure and performance in Telecommunications", document de travail n 237 de l'OCDE, Paris.

Brown, D. et Stern, R. (2001), "Measurement and Modelling of the Economic Effects of Trade and Investment Barriers in Services", Review of International Economics, vol. $9 n^{\circ} 2$.

Brown, D., Deardorff, A. et Stern, R. (1996), "Modelling Multilateral Liberalisation in Services", AsiaPacific Economic Review 2, pp. 21-34.

Brown, D., Deardorff, A. et Stern, R. (2000), "CGE Modelling and an Analysis of Multilateral and Regional Negotiating Options", document établi pour la conférence donnée sur les problèmes et options concernant les politiques commerciales des États-Unis et du Japon à l'échelle multilatérale, régionale et bilatérale, University of Michigan, Gerard R. Ford School of Public Policy and Japan Economy Program, Department of Economics, 5 et 6 octobre.

Chadha, R. (2000), "GATS and the Developing Countries: A Case Study of India", à paraître dans Robert M. Stern (éd.), Services in the International Economy: Measurement and Modeling, Sectoral and Country Studies, and Issues in the WTO Services Negotiations, University of Michigan Press, Ann Arbor, Michigan.

Chadha, R., Brown, D., Deardorff, A. et Stern, R. (2000), “Computational Analysis of the Impact on India of the Uruguay Round and the Forthcoming WTO Trade Negotiations", Document de réflexion n459, School of Public Policy, University of Michigan. Ann Arbor, Michigan.

Chen, Le and Sherif, M. (2002), “China's Foreign Economic Cooperation Development: Exporting Chinese Construction Services", Griffith University, Australie.

Chen, Z. et Schembri, L., (2002), « Mesure des obstacles au commerce des services : études et méthodes », Ministère des Affaires étrangères et du Commerce international, Canada.

Claessens S., Demirgüç-Kunt A. et Huizinga, H. (2001), "How does Foreign Entry Affect Domestic Banking Markets?” Journal of Banking and Finance 25, pp. 891-911. 
Claessens, S. et Glaessner, T. (1998), "Internationalization of Financial Services in Asia”, Banque mondiale.

Clark, X., Dollar D. et Micco A.( 2002), "Maritime Transport Costs and Port Efficiency“, Banque mondiale.

CNUCED (1999), « Analyse de l'expérience dans certains secteurs de services », Genève.

CNUCED (2000), "Report of the Expert Meeting on National Experiences with Regulation and Liberalization: Examples in the Construction Services Sector and its Contribution to the development of Developing Countries", Conseil du commerce et du développement, Commission du commerce des biens et services, et des produits de base, cinquième session, Genève 19-23 février 2001. TD/B/COM.1/32

CNUCED (20001), "From Selling Sheep to Transcribing Tapes", communiqué de presse TAD/INF/PR/35, daté du 20 novembre 2001.

CNUCED, OMS, (1998), International Trade in Health Services: A Development Perspective, Genève.

Colecchia, A. (2001), "Measuring barriers to market access for services: A pilot study on accountancy services", in Findlay, C. et Warren, T. (éds.), Impediments to Trade in Services: Measurement and Policy Implication. New York: Routledge.

Commission économique et sociale pour l'Asie et le Pacifique (CESAP) (2000), "Major Issues in Transport, Communications, Tourism and Infrastructure Development: Regional Shipping and Port Development Strategies under a changing maritime environment", Comité des transports, des communications, du tourisme et du développement infrastructurel, troisième ession, 15-17 novembre, Bangkok.

Commission européenne, CNUCED, FMI, Nations unies, OCDE, OMC (2002), Manuel des statistiques of du commerce international des services, disponible sur www.wto.org

Deardoff, A. (2001), "International Provision of trade Services, Trade and Fragmentation", Review of International Economics, 9(2), pp.233-248.

Deardorff, A. et Stern, R. (1998), "Measurement of Nontariff Barriers”, Ann Arbor: University of Michigan Press

Dee, P. et Hanslow, K. (2000), "Multilateral Liberalisation of Services Trade", Productivity Commission Staff Research Paper, Ausinfo, Canberra.

Deloitte Touche Tohmatsu (2003), "Global Powers of Retailing: from source to consumer", disponible sur www.stores.org.

Doove, S., Gabbitas, O., Nguyen-Hong, D., et Owen, J. (2001), "Price Effects of Regulation: Telecommunications, Air Passenger Transport and Electricity Supply", Productivity Commission Staff Research Paper, AusInfo, Canberra.

Eschenbach, F. et Francois, J. (2002), "Financial Sector Competition, Services Trade, and Growth," Documents de réflexion du CEPR 3573. 
Findlay, C. et Warren, T. (2000), "Impediments to Trade in Services: Measurement and Policy Implication", New York: Routledge.

Fink, C., Mattoo, A., et Neagu, I. (2001), "Trade in International Maritime Services: How Much Does Policy Matter?”, Banque mondiale.

Fink, C., Mattoo, A., et Rathindran, R.(2002), Liberalizing Basic Telecommunications: Evidence from Developing Countries, document présenté à la réunion des experts en services de l'OCDE et de la Banque mondiale qui s'est tenue à Paris les 4 et 5 mars 2002.

Francois, J. et Hoekman, B. (1999), "Market Access in the Service Sectors”, manuscrit, Tinbergen Institute.

Francois, J. et Schuknecht, L. (2000), "International Trade in Financial Services, Competition, and Growth Performance", Centre for International Economic Studies no. 6.

Francois, J. et Wooton, I. (2000), "Market Structure, Trade Liberalization and the GATS“, Tinbergen Institute, 2000

Francois, J. et Wooton, I. (2001), "Trade in International Transport Services: the Role of Competition", Review of International Economics 9(2).

Francois, J., Van Meijl, H. et van Tongeren, F. (2002), "Economic Benefits of the Doha round for the Netherlands", polycopié.

Hardin, A. et Holmes, L. (1997), "Services Trade and Foreign Direct Investment, Staff Research Paper, Industry Commission. Canberra: Services de publication du gouvernement australien.

Hong Kong Coalition of Services Industries, “Hong Kong Services 2000”, Hong Kong.

Jensen, J., Rutherford, T. et Tarr, D. (2002), “Economy-Wide Effects of Russia's Accession to the WTO”, document élaboré pour la conférence ASDI-CEFIR sur les négociations relatives à l'accession de la Russie à l'OMC, juin 2002.

Johnson, M., Gregan, T., Gentle, G. et Belin, P. (2000), "Modelling the benefits of increasing competition in international air services" in Findlay, C et Warren, T (eds.), Impediments to Trade in Services: Measurement and Policy Implication, New York: Routledge.

Jomini, P., Verikios, G. et Zhang, X. (2002), "Quantifying the Effects of Liberalizing Services: the Experience of the Australian Productivity Commission”, réunion des experts en services de 1'OCDE et de la Banque mondiale, Paris (4 et 5 mars 2002).

Kalirajan, K. (2000), "Restrictions on Trade in Distribution Services”, Productivity Commission Staff Research Paper, Ausinfo, Canberra.

Kalirajan, K., McGuire, G., Nguyen-Hong, D. et Shuele, M. (2000), "Restrictiveness of International Trade in Banking Services", in Findlay, C. et Warren, T. (eds.), Impediments to Trade in Services:

Measurement and Policy Implication, New York: Routledge.

Kang, J. (2000), "Price Impact of Restrictions on Maritime Transport Services", in Findlay, C. et Warren, T. (eds.), Impediments to Trade in Services: Measurement and Policy Implication. New York: Routledge. 
Kemp, S. (2000), "Trade in Education Services and the Impacts of Barriers to Trade", in Findlay C. et Warren T. (eds.), Impediments to Trade in Services: Measurement and Policy Implication. New York: Routledge.

Konan, D. et Maskus, K. (2002), "Quantifying the impact of services liberalisation in a developing country”, document présenté à la neuvième conférence annuelle de l'ERF (Economic Research Forum), en octobre 2002.

Marko, M. (1998), “An Evaluation of the Basic Telecommunications Service Agreement”, Policy Discussion Paper No. 98/09, Centre for International Economic Studies, University of Adelaide, Australie.

Markusen, J. et Rutherford T. (2002), "Developing domestic entrepreneurship and growth through imported expertise", CEBR Discussion paper No 2002-12.

Markusen, J., T. Rutherford et D. Tarr (2000), "Foreign Direct Investment in Services and the Domestic Market for Expertise", Policy Research Paper No. 2413, Banque mondiale, Washington DC, août.

Mattoo, A. (1998), "Financial Services and the World Trade Organization: Liberalization Commitments of the Developing and Transitional Economies," manuscrit (document interne), Banque mondiale.

Mattoo, A., Rathindran, R. et Subramanian, A. (2001), "Measuring Services Trade Liberalization and its Impact on Trade Growth: An Illustration", document de travail 2655 de la Banque mondiale.

McGuire G. (2002a), "How Important are Restrictions on Trade in Services?”, document de référence présenté à la réunion des experts en services de l'OCDE et de la Banque mondiale, OCDE, Paris (4 et 5 mars 2002).

McGuire, G. (2002b), "Methodologies for Measuring Restrictions on Trade in Services", document présenté à la réunion des experts en services de l'OCDE et de la Banque mondiale, OCDE, Paris (4 et 5 mars 2002).

McGuire, G. et Schuele, M. (2000), "Restrictiveness of International Trade in Maritime Services", in Findlay C. et Warren T. (eds.), Impediments to Trade in Services: Measurement and Policy Implication, New York: Routledge.

Ministère australien des Affaires étrangères et du Commerce (1999), Global Trade Reform: Maintaining Momentum, Canberra.

Mukherjee, A. (2001), "India's Trade in Maritime Transport Services under the GATS Framework", Indian Council for Research on International Economic Relations (ICRIER), Delhi.

Mukherjee, A. (2001a), "Trade in Construction and Consultancy Services: India and the GATS", Indian Council for Research on International Economic Relations (ICRIER), Delhi.

Mukherjee, A. (2002), "India's Trade Potential in Audio-visual Services and the GATS”, Indian Council for Research on International Economic Relations (ICRIER), Delhi.

Mukherjee, A. (2002a), "Distribution services: India and the GATS 2000 negotiations", Indian Council for Research on International Economic Relations, Delhi. 
Nguyen-Hong, D. (2000), "Restrictions on Trade in Professional Services," Productivity Commission Staff Research Paper, Ausinfo, Canberra.

Norton, S. (1992), "Transaction Costs, Telecommunications, and the Microeconomics of Macroeconomic Growth" Economic Development and Cultural Change, 41(1), pp. 175-96.

OCDE (1999), Les services stratégiques aux entreprises, OCDE, Paris.

OCDE (2000), « Summary Indicators of Product Market Regulation with an Extension to Employment Protection Legislation », document de travail n²26, Département des Affaires économiques, ECO/WKP(99)18, OCDE, Paris.

OCDE (2001), Revue économique, $\mathrm{n}^{\circ} 32$, Paris.

OCDE (2002), Statistics on International Trade in Services: Partner Country Data and Summary Analysis 1999-2000, OCDE, Paris.

Organisation panaméricaine de la santé, OMS (2002), Trade in Health Services: global, regional, and country perspectives, Genève.

PricewaterhouseCoopers (2002), "Doing Business and Investing in South Africa”, Information Guide Series, disponible sur www.pwc.global.com.

PricewaterhouseCoopers (2003), "Doing Business in Tanzania", disponible sur www.pwcglobal.com.

PricewaterhouseCoopers (2003a), "Ports and Airports", Government of Kenya Conference on PSP in Infrastructure, 15 Mai.

Robinson, S., Wang, Z et Martin, W. (1999), "Capturing the Implications of Services Trade Liberalisation", document présenté lors de la deuxième conférence annuelle sur le thème "Global Economic Analysis”, Ebberuk, Danemark, 20-22 juin.

Rodriguez, F. et Rodrick, D. (1999), “Trade Policy and Economic Growth: a Skeptic's Guide to CrossNational Evidence", CEPR DP 2143.

Rodrik, D. (2002), “Feasible Globalizations”, NBER Working Paper, No. W9129, août 2002.

Roller, L. et Waveman, L. (2001), "Telecommunication Infrastructure and Economic Development: A Simultaneous Approach", American Economic Review, Vol. 91, n 4.

Trewin, R. (2001), “A Price-Impact Measure of Impediments to Trade in Telecommunication Services” in Findlay C. et Warren T. (eds.), Impediments to Trade in Services: Measurement and Policy Implication. New York: Routledge.

Verikios, G. et Zhang, X. (2000), "Sectoral Impact of Liberalising Trade in Services”, document présenté à la troisième conférence organisée sur le thème "Global Economic Analysis", Melbourne, 27-30 juin,. Disponible sur: www.monash.edu.au/policy/conf/53Verikios.pdf

Verikios, G. et Zhang, X. (2001), "Global Gains from Liberalising Trade in Telecommunications and Financial Services", Productivity Commission Staff Research Paper, AUSInfo, Canberra. Paper disponible sur http://www.pc.gov.au/research/staffers/ggflt/index.html 
Warren, T. (2000), "The Impact on Output of Impediments to Trade and Investment in Telecommunications Services," in Findlay, C. et Warren, T. (eds), Impediments to Trade in Services: Measurement and Policy Implications, New York: Routledge

Winters, A. (2001), “Assessing the Efficiency Gain for Further Liberalization: A Comment”, in Sauvé, P. et Subramaniam, A. (eds.), Efficiency, Equity and Legitimacy: The Multilateral Trading System at the Millenium, Chicago University Press, Chicago, 2001.

Winters, A. (2002), "The Economic Implications of Liberalizing Mode 4 Trade", Joint WTO-World Bank Symposium on 'The Movement of Natural Persons (Mode 4) under the GATS', WTO, Genève, 11-12 avril.

Winters, A., Walmsley, T., Wang, Z. H. et Grynberg, R. (2002), "Negotiating the Liberalisation of the Temporary Movement of_Natural Persons",Document de réflexion 87, University of Sussex, octobre. 